\title{
QUANTIFICATION OF THE ENHANCEMENT FACTOR IN \\ SURFACE-ENHANCED RAMAN \\ SCATTERING
}

by

Evan Blackie

\begin{abstract}
A thesis
submitted to the Victoria University of Wellington

in fulfilment of the requirements for the degree of

Doctor of Philosophy

in Chemistry
\end{abstract}

Victoria University of Wellington

The MacDiarmid Institute for Advanced Materials and Nanotechnology

2010 

In loving memory of

Jonathan Robert Stuart Blackie

(1948 - 1990) 



\section{ACKNOWLEDGEMENT}

This thesis "emerged" from the collective help, support and direction of many different people, including the NZ taxpayer via the MacDiarmid Institute and Victoria University. I would like to particularly acknowledge several people.

First and foremost, I am deeply indebted to Pablo Etchegoin, without whose scientific expertise, strategic direction, and mentoring, I would not have got this far. I am also deeply grateful to Eric Le Ru as a supporting supervisor, physics tutor, and general troubleshooter, for help with much of the analytical and theoretical work included herein. I consider it truly a privilege to have worked in such an encouraging and academically engaging environment.

I also thank my colleagues Matthias Meyer, Chris Galloway, Stefan Meyer, who have been friendly, open-minded and supportive friends in my journey, as well as on many occasions physics tutors. I can only apologize for the limited benefit I have been in making up the requisite numbers in lunchtime soccer! I acknowledge in particular Matthias Meyer, who has unceasingly been available to help with computing and software queries, and Chris Galloway, who has been a soundboard for sharing physical ideas.

As a researcher in an interdisciplinary environment, much of this work would not be possible without the participation of many others. Here, I acknowledge the wider support network of academics and colleagues at SCPS. In the physics world, these include Joe Trodahl, Ben Ruck, Chris Brumby, Andy Preston, and Bart Ludbrook - an interesting and learned bunch to engage with at many different levels. In the chemical realm, I thank Peter Northcote, Brendan Burkett, Mattie Timmer, the Marine lab (particularly John Ryan and Jonathan Singh), the Organic Synthesis lab (thanks to Russell Frew), and the Organometallic lab. It has been great to maintain my roots in the alchemy profession! Finally I thank Richard Tilley, John Spencer and Kate McGrath for general advice and support.

No less important in enabling my research have been the general staff and stores personnel that have kept things ticking along in the background. Here, I thank Margaret Brown, Darren Alexander, Scott Forbes, Alan Rennie, Grant Franklin, 
Sally Wisheart, Helen Rowley, Gordon Heeley, and Lisa Meredith.

Finally, I thank personally friends and family who have given me support in times of doubt, particularly my Mum with her constant encouragement and belief, and fellow $\mathrm{PhD}$ companion Benjamin Matthewson, who has paved the way forward. 


\section{Abstract}

This thesis presents a rigorous stepwise methodology towards the accurate measurement and quantification of the SERS enhancement factor (EF), the key parameter in describing the SERS effect. The work represents, we believe, a successful attempt to resolve some of the inconsistencies in the literature and to refocus the field by emphasizing the importance of consistent definitions and rigorous quantification to elucidate matters of fundamental importance in SERS. The success in our approach is that it combines careful experimental measurements upon a sound theoretical framework, and utilizes a 'toolbox' of techniques developed in recent years, such as bi-analyte SERS (BiASERS) techniques for single-molecule (SM) detection, and isotopic editing.

In experimental work, we measure the bare Raman cross-sections of five common probes used in SERS as a first step in measuring the analytical enhancement factor (AEF) and single-molecule enhancement factor (SMEF). The methodology in measuring these EFs involved the use of a reference standard of known crosssection along with a careful characterization of the scattering volume through beam profiling experiments. As a guide to validating the reference cross-section we make extensive use of density functional theory (DFT) calculations to obtain estimates for the intrinsic Raman cross-sections of small, non-resonant probes.

The results of this work showed that previous upper limits for the EF reported in the literature of $10^{14}$ were based on a faulty normalization of the EF. In fact, EFs of $10^{8}$ were sufficient to see single molecules, which is much lower than previously expected; under optimum conditions, even lower EFs, possibly down to $10^{5}$ could be sufficient for the SM detection of resonant probes.

As a valuable extension of BiASERS, we elaborate on the synthesis of isotopic analogues of a rhodamine dye as ideal partners for SM experiments. The synthesis and definitive characterization of these probes enable their use in an experiment to determine the SM regime in a liquid colloidal sample. Isotopically edited dyes such as these, in combination with the methodologies of EF quantification outlined herein, set the standard for those interested in accurate quantification of the 
SERS effect. This approach is useful in terms of both basic theoretical questions and applications such as the effective comparison of SERS substrates.

Finally, we extend the techniques developed over the thesis to a long-standing and largely unresolved question in SERS: What is the minimum intrinsic Raman cross-section that can be measured as a single molecule in standard SERS conditions. In this work, we explore the SM detection non-resonant probes, which are the molecules of interest for many practical applications such as forensics and biological assays. Specifically, we demonstrate the successful SM detection of isotopically edited adenine probes. 


\section{Contents}

1 INTRODUCTION 9

1.1 Quantifying the SERS Enhancement Factor $(\mathrm{EF}) \quad \ldots \ldots$

2 BACKGROUND 11

2.1 Basic Raman Theory . . . . . . . . . . . . . . . . . . 11

2.1.1 Raman scattering . . . . . . . . . . . . . . 12

2.1.2 Resonance Raman scattering . . . . . . . . . . . . . . . 12

2.1.3 The Raman process in more detail . . . . . . . . . . . 13

2.1.4 Important Raman parameters . . . . . . . . . . . . . . . 16

2.1.5 Semi-classical approach to Raman cross-sections . . . . . 18

2.1.6 Density functional theory $(\mathrm{DFT}) \ldots \ldots$. . . . . . 20

2.2 Surface-Enhanced Raman Spectroscopy (SERS) . . . . . . . . . . 21

2.2 .1 Discovery of SERS . . . . . . . . . . . . . 22

2.2.2 Electromagnetic enhancement . . . . . . . . 23

2.2.3 Chemical enhancement . . . . . . . . . . . 28

2.2 .4 A note on surface adsorption . . . . . . . . . . . 29

2.2 .5 Other mechanisms . . . . . . . . . . . . . . . 29 


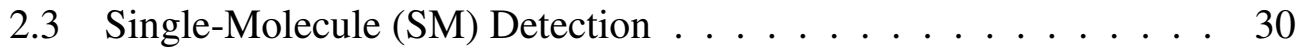

2.3.1 Background ................ 30

2.3.2 Bi-analyte SERS (BiASERS) . . . . . . . . . 32

2.4 The SERS Enhancement Factor . . . . . . . . . . . . . . . . 33

2.4.1 The single-molecule enhancement factor (SMEF) . . . . . 34

2.4.2 The SERS substrate enhancement factor (SSEF) . . . . 35

2.4.3 The analytical enhancement factor $(\mathrm{AEF}) \ldots \ldots$

2.5 Vibrational Pumping in SERS . . . . . . . . . . . . 37

3 EXPERIMENTAL 43

$3.1 \quad$ Raman Setup . . . . . . . . . . . . . . . . . . 43

3.2 Analytes used in Raman/SERS experiments . . . . . . . . . . . . 46

3.3 Substrate Preparation . . . . . . . . . . . . . . . . 46

3.3.1 Synthesis of silver colloids . . . . . . . . . . . . 46

3.3.2 Preparing liquid colloidal aggregates . . . . . . . . . . 48

3.3.3 Preparing solid colloidal substrates . . . . . . . . . . . 49

3.4 Supporting Experimental Techniques . . . . . . . . . . . . . . 51

3.4 .1 UV-Vis spectroscopy . . . . . . . . . . . . 51

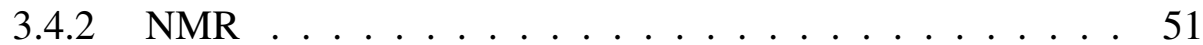

3.4.3 Mass spectroscopy . . . . . . . . . . . . 52

3.4.4 TDVP measurements . . . . . . . . . . . . 52

3.4.5 DFT calculations ................ 52

3.5 Synthesis and Characterization of Isotopic Dyes . . . . . . . . 53 
4 ESTIMATION OF RAMAN CROSS-SECTIONS

4.1 Motivation . . . . . . . . . . . . . . 55

4.1.1 The EF problem ................. 55

4.1.2 Previous measurements of EFs . . . . . . . . . 56

4.1 .3 Our approach . . . . . . . . . . . . . 58

4.1.4 Why measure the actual Raman cross-section? . . . . . . 58

4.2 Measurement Methodology . . . . . . . . . . . . . . . . . 60

4.2 .1 Use of a reference . . . . . . . . . . . 60

4.2.2 Selection of a reference . . . . . . . . . . . 61

4.2.3 Validation of reference using small molecules . . . . . . 66

4.3 Raman Measurement of SERS Probes . . . . . . . . . . . . . 67

4.3.1 Analytes of interest . . . . . . . . . . . 67

4.3.2 Measurement of Raman cross-sections . . . . . . . . . . 68

4.4 Discussions . . . . . . . . . . . . . . 73

4.4.1 Comparison with the literature . . . . . . . . 73

4.4 .2 General remarks . . . . . . . . . . . . . 74

4.5 Conclusions . . . . . . . . . . . . . . . 75

5 QUANTIFYING THE SERS EF

5.1 Background ...................... 77

5.2 EF Definitions . . . . . . . . . . . . . . . . . 78

5.2 .1 Considerations . . . . . . . . . . . . 78

5.2.2 SERS substrates in this work . . . . . . . . . 79 
5.2.3 Specific EF definitions . . . . . . . . . . . . 79

5.2 .4 Experimental approach . . . . . . . . . . . 80

5.3 Experimental Measurement of AEFs . . . . . . . . . . . . 81

5.4 Discussion of the AEFs . . . . . . . . . . . . . . . . . 84

5.4.1 General observations . . . . . . . . . . . . . 84

5.4.2 Variation of AEFs amongst vibrational modes . . . . . . 86

5.5 Experimental Measurement of SMEFs . . . . . . . . . . . . . . . 89

5.5.1 Identifying SM events and obtaining the SMEF . . . . . 90

5.5.2 SMEFs in aggregated colloidal solutions . . . . . . . 91

5.5.3 SMEFs in fixed colloidal structures . . . . . . . . . 94

5.5.4 Measuring the SMEF using temperature-dependent vibrational pumping (TDVP) . . . . . . . . . . . . 97

5.6 Discussion of the SMEFs . . . . . . . . . . . . . . 101

5.7 Conclusions . . . . . . . . . . . . . . . . 103

6 ADVANCED BIASERS 107

6.1 Isotopic Editing in SERS . . . . . . . . . . . . . . . . 108

$6.1 .1 \quad$ Previous studies . . . . . . . . . . . . 108

6.1.2 Rhodamines . . . . . . . . . . . . 110

6.2 Synthetic Strategy for Isotopic Dyes . . . . . . . . . . . . . 111

6.2 .1 Initial attempts $\ldots \ldots \ldots \ldots \ldots$

6.2.2 Ethylation of free amine using ethyl iodide . . . . . . 112

6.2.3 Esterification of free acid using ethyl iodide . . . . . . . . 114 
6.2.4 Ring-forming condensation reaction (Friedel-Crafts reaction) . . . . . . . . . . . . . . . . 114

6.3 Optical Characterization of Isotopic Dyes . . . . . . . . . . 116

6.3.1 Fluorescence and extinction measurements . . . . . . 116

6.3.2 Bare (non-SERS) Raman spectra and cross-sections . . . . 117

6.3.3 Use in individual SERS experiments . . . . . . . . . . . 121

6.4 Single-Molecule (SM) Experiments . . . . . . . . . . . . . 122

6.4.1 BiASERS experiments . . . . . . . . . . 122

6.4.2 Probing the SM regime in liquids . . . . . . . 126

6.5 Discussion . . . . . . . . . . . . . . . . . . . . 129

6.5.1 General observations . . . . . . . . . . . . . . . 129

6.5.2 Scope of reaction methodology . . . . . . . . . 130

6.5.3 Other strategies for obtaining deuterated samples . . . . 131

6.6 Conclusions . . . . . . . . . . . . . . . . . . . 132

7 NON-RESONANT PROBES 135

7.1 Introduction . . . . . . . . . . . . . . . . . 135

7.1.1 Minimum cross-section measurable as a SM . . . . . . 136

7.1 .2 Previous reports . . . . . . . . . . 137

7.2 Method for SM detection of Non-Resonant Probes . . . . . . . . 139

7.3 Experimental Details . . . . . . . . . . . . . . . . 143

7.3.1 Substrate optimization . . . . . . . . . . . . 143

7.3.2 SERS experiments . . . . . . . . . . . . . 144

7.3.3 Linear deconvolution analysis . . . . . . . . . . 144 
7.3.4 Optimizing laser conditions _. . . . . . . . . . . 145

7.3.5 Choice of the non-resonant probes . . . . . . . . . . 146

7.3.6 Initial experimental attempts . . . . . . . . . . . 147

7.4 Measurement of BPE/BTZ System . . . . . . . . . . . . . . . 148

7.4.1 Basic properties of probes . . . . . . . . . . . 149

7.4.2 Measurement of bare Raman cross-sections . . . . . . . . 150

7.5 Results and Discussion for BTZ/BPE System . . . . . . . . . 151

7.5.1 BiASERS experiments . . . . . . . . . . 151

7.5 .2 SMEFs for BPE $\ldots \ldots \ldots \ldots 155$

7.6 Results and Discussion for Adenine System . . . . . . . . . . 157

7.6.1 BiASERS and SMEFs for adenine . . . . . . . . . 158

7.7 Conclusions . . . . . . . . . . . . . . . . . 159

8 CONCLUSIONS 163

A MEASURING THE SCATTERING VOLUME 169

A.1 Theoretical Considerations . . . . . . . . . . . . . . . . . . 169

A.1.1 The need to characterize the scattering volume . . . . . 169

A.1.2 General Principles . . . . . . . . . . . . . . 170

A.2 Excitation Profile . . . . . . . . . . . . . . . 171

A.3 Detection Efficiency Profile . . . . . . . . . . . . . . 171

A.4 Beam Profiling Experiments . . . . . . . . . . . . . . . 173

A.4.1 Scan over an edge . . . . . . . . . . . 173

A.4.2 Slit size dependence . . . . . . . . . . . . . 175 
A.4.3 Axial detection efficiency . . . . . . . . . 176

A.5 Obtaining the SMEF . . . . . . . . . . . . . . 179

A.5.1 Calculating the SMEF from SM-SERS event . . . . . . 179

A.5.2 Calculating the SMEF from TDVP . . . . . . . . . 180

A.6 Spectral Analysis . . . . . . . . . . . . . . . . . 181

A.6.1 Least-squares fitting to pseudo-Voigt function . . . . . . 182

A.6.2 Linear least-squares deconvolution . . . . . . . . . . 182

A.6.3 Modified principal component analysis (MPCA) . . . 183

B RAW SPECTRAL DATA 190

$\begin{array}{ll}\text { C PUblications } & 197\end{array}$ 


\section{Chapter 1}

\section{INTRODUCTION}

\subsection{Quantifying the SERS Enhancement Factor (EF)}

This thesis covers a body of work centred on the accurate quantification of the SERS enhancement factor (EF), a critical parameter in describing the SERS effect for theory and applications alike. In the following, we provide a brief outline of the structure of the thesis:

Chapter 2 of the thesis begins by outlining the theory necessary to understand the work that follows along with some of the Raman and SERS history. Chapter 3 gives the experimental details of the Raman microscope used in our studies along with substrate preparation and synthetic details.

Chapter 4 describes the first step in accurate quantification of the SERS EF, namely, measurement of the non-SERS Raman signal to enable correct normalization of the EF. The necessary theoretical background for this method is described and previous work and its failings are analysed. Ultimately, the non-SERS Raman cross-sections are measured for some probes of common interest in the SERS field.

Following on from this, Chapter 5 extends the work to estimation of the SERS EF. The chapter starts by identifying suitable definitions for the EF itself and 
outlining the experimental approach we intend to take. The measurement of two forms of enhancement factor, the analytical enhancement factor (AEF) and the single-molecule enhancement factor (SMEF), are performed for the common probes measured in Chapter 4. The results of this chapter form the backbone of the thesis and are, we believe, the best estimates to date of SMEFs.

Chapter 6 represents a departure from the general methods used up until this point and details our work in synthesizing isotopically edited versions of a common rhodamine dye as ideal probes in bi-analyte SERS (BiASERS) experiments. The synthesis and subsequent characterisation of these dyes is described in detail, before their application in a BiASERS experiment. This experiment nicely demonstrates the transition from the single-molecule (SM) to the many-molecule regime upon changing acquisition time in a liquid colloidal sample. The synthesized dyes are shown to be ideal for such experiments.

The final chapter, Chapter 7, is a culmination of the work of the preceding chapters and explores the SM detection of non-resonant probes, which, by definition, are much harder to detect than the resonant probes used typically in fundamental SERS studies. After thorough optimization of the SERS colloidal substrate, we demonstrate the successful detection of SM events of non-resonant probes, first for a case of a molecule with a moderate Raman cross-section (1,2-di-(4pyridyl)-ethylene), and later for a probe with a low Raman cross-section, for the biologically relevant probe adenine. In this latter case, we make use of isotopically edited adenine partners in BiASERS, thus bringing together many of the elements discussed previously.

The results of this thesis, and the impact on the field are then presented in a detailed conclusions chapter. The impact on applications along with some future applications are also discussed. 


\section{Chapter 2}

\section{BACKGROUND}

We present here some of the history and theoretical background of Raman and SERS to support the work that follows. Included in this material are descriptions of the major techniques we will use in our approach, including bi-analyte SERS (BiASERS) for single-molecule (SM) detection, and temperature dependent vibrational pumping (TDVP).

\subsection{Basic Raman Theory}

The best place to learn about the Raman process in any detail is via a textbook or similar review, and naturally the treatment can be extended to any desired level of complexity, from a basic classical approach to a full quantum electrodynamics treatment. Accordingly the information here will limit the description of Raman theory to the level required to understand the work in this thesis. Readers interested in further details are directed to Refs. $[1,2,3]$. 


\subsubsection{Raman scattering}

Simply put, Raman scattering is the inelastic scattering of light due to interaction with the vibrational states, or modes, of a molecule. The effect was postulated theoretically by A. Smekal et al. in 1923 [4] but was first discovered experimentally by C.V. Raman [5] in 1928 in an experiment using the sun as a light source and some basic optical filters.

The scattering process involves the instantaneous absorption and emission of a photon by a molecule. As the energy level diagram shows in Fig. 2.1, three different types of scattering are possible:

(i) Rayleigh scattering: the wavelengths of the absorbed and emitted photons are the same;

(ii) Stokes scattering: the emitted photon is red shifted with respect to the ground state (the molecule moves to a more vibrationally excited state of the ground electronic state $S_{0}$ ); and

(iii) anti-Stokes scattering: the emitted photon is blue shifted with respect to the ground state (the molecule moves to a less vibrationally excited state).

Note in this diagram, we refer to a virtual state. In fact, there is no physical reality to this state but it is helpful for understanding the process and provides a link to the conception of Raman in a quantum mechanical context.

\subsubsection{Resonance Raman scattering}

An additional phenomena in Raman, which becomes relevant in SERS also, occurs when the energy of the incident photons (and scattered) is at the level of the electronic transitions of the molecule (see Fig. 2.2). In this case, the virtual state becomes resonant with one of the real electronic levels in the molecule, resulting in an enhancement in the scattering efficiency of the molecule of up to several orders of magnitude. Any molecule that absorbs in the vicinity of the incident laser 


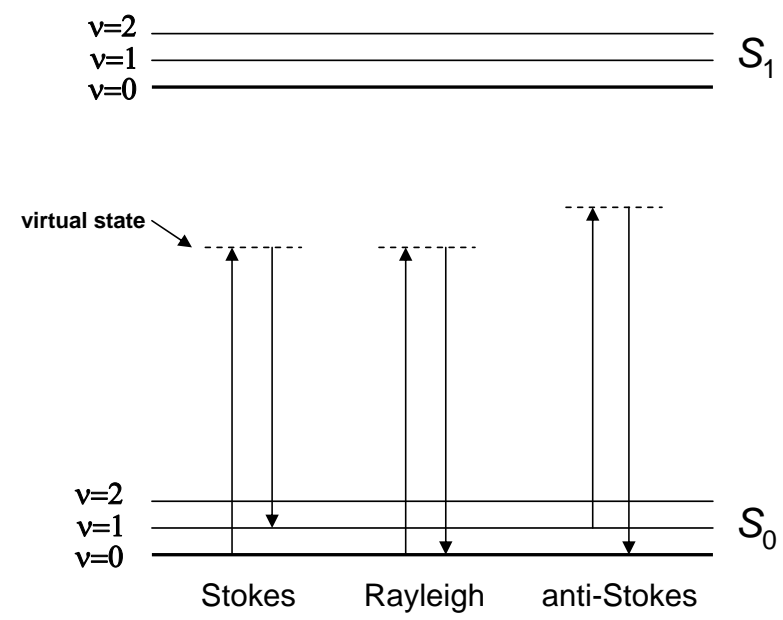

Figure 2.1: Simplified Jablonski diagram of Raman process. Note that the virtual state is not a real physical state.

wavelength is subject to this Resonance Raman Scattering (RRS). Many common probes in SERS (such as the dyes Rh6G, CV) are used exactly because they have a resonance Raman contribution in the visible and accordingly have large Raman cross-sections. In fact, work in later chapters will show the difficulties in extending SERS beyond resonant analytes.

\subsubsection{The Raman process in more detail}

\section{Dipolar emission model}

Within classical EM theory, radiation of light from a localized source can be described in terms of its multipolar components, with the dominant contribution that of the dipole. A standard approach to the Raman effect from a classical optics perspective is to consider Raman scattering as the induction of a Raman dipole within the molecule by an incident electric field, followed by the subsequent radiation of scattered light from this Raman dipole. 


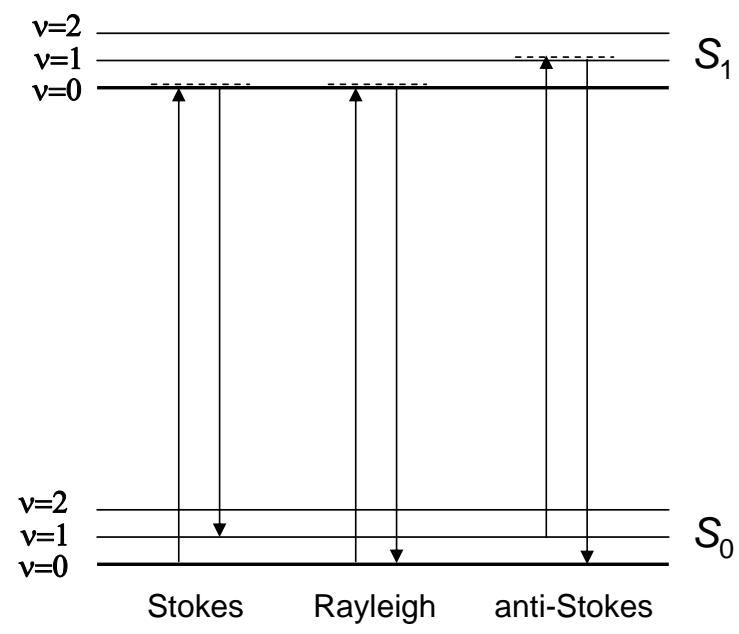

Figure 2.2: Simplified Jablonski diagram of resonance Raman scattering (RRS), which results in an enhancement of the efficiency of the Raman process. Many of the common dyes used in SERS are utilized because they have large cross-sections as a result of RRS.

\section{The linear optical polarizability tensor}

To express this process mathematically, we begin by defining the linear optical polarizability, $\hat{\alpha}_{L}(\omega)$, which relates the induced dipole of a molecule, $\underline{\mathbf{p}}_{L}(\omega)$, oscillating at the same frequency, $\omega$, as the incident electromagnetic field of the laser $\underline{\mathbf{E}}(\omega)$ as:

$$
\underline{\mathbf{p}}_{L}(\omega)=\hat{\alpha}_{L}(\omega) \cdot \underline{\mathbf{E}}(\omega),
$$

where the function is expressed in complex notation and $\hat{\alpha}_{L}(\omega)$ is the optical polarizability tensor, which fully characterizes the linear optical response of the molecule.

\section{The Raman polarizability tensor}

If we now consider inelastic scattering, in this case for Stokes Raman scattering, we have a photon scattered at a frequency $\omega_{R}=\omega_{L}-\omega_{v}$. In a similar manner to 
above, we can express the induced dipole from the scattered radiation as:

$$
\underline{\mathbf{p}}_{R}\left(\omega_{R}\right)=\hat{\alpha}_{R}\left(\omega_{L}, \omega_{v}\right) \cdot \underline{\mathbf{E}}\left(\omega_{L}\right),
$$

where $\hat{\alpha}_{R}\left(\omega_{L}, \omega_{\nu}\right)$ is the Raman polarizability tensor dependent on both the laser and mode frequencies, a fully phenomenological parameter in this sense. If $\hat{\alpha}_{R}\left(\omega_{L}, \omega_{v}\right)$ is specified for a given vibrational mode, then the Raman scattering process can be fully described classically as the induction of a Raman dipole, which radiates the Raman scattered light.

\section{The Raman tensor}

From the perspective of molecular vibration, we consider the perturbation in the linear optical polarizability due to the presence of vibrations. These vibrations can be characterized by normal coordinates $Q_{k}$, which describe the scalar amplitude of the deformation according to the pattern of a given normal mode $k$. Taking a specific normal mode $k$ characterized by $Q_{k}$ and considering the perturbation in the linear optical polarizability, we obtain the following Taylor expansion:

$$
\hat{\alpha}_{L}\left(Q_{k}\right)=\hat{\alpha}_{L}(0)+\left(\frac{\partial \hat{\alpha}_{L}}{\partial Q_{k}}\right)_{Q_{k}=0} Q_{k}+\frac{1}{2}\left(\frac{\partial^{2} \hat{\alpha}_{L}}{\partial Q_{k}^{2}}\right)_{Q_{k}=0} Q_{k}^{2}+\ldots
$$

Here, the first term represents the unperturbed linear optical polarizability (or the Rayleigh scattering contribution) and the later terms are its first- and second-order perturbations. The Raman tensor, $\hat{R}_{k}$, of the normal mode $k$, is then defined as:

$$
\hat{R}_{k}\left(\omega_{L}\right)=\hat{\alpha}_{i}^{\prime}=\left(\frac{\partial \hat{\alpha}_{L}\left(\omega_{L}\right)}{\partial Q_{k}}\right)_{Q_{k}=0}
$$

That is, the Raman tensor is a measure of the change in linear polarizability when the molecule is deformed according to the deformation pattern of normal mode $k$. It can be related to the Raman polarizability tensor in Eqn. 2.2 by: 


$$
\hat{\alpha}_{k}\left(\omega_{L}\right)=\frac{Q_{k}^{0}}{2} \hat{R}_{k}\left(\omega_{L}\right)
$$

This equation then links the fully phenomenological description of Raman in terms of a Raman polarizability tensor with the microscopic interpretation of scattering by vibrational modes [2].

\section{Raman selection rules}

The existence of a given vibrational mode in a molecule does not necessarily guarantee Raman scattering for that mode, which gives rise to the Raman selection rules.

Stated formally, if $\hat{\alpha}_{L}(\omega)$ does not depend on $Q_{k}$ in Eqn. 2.4 (a common situation in a molecule with high symmetry), then $\hat{R}_{k}\left(\omega_{L}\right)=0$ and no Raman scattering is observed for that mode, i.e. it is Raman inactive. This may arise when the contribution of some groups of atoms is exactly cancelled out by an identical group with the same pattern of vibration due to symmetry.

\subsubsection{Important Raman parameters}

\section{The Raman frequency}

In Raman experiments, the primary parameter that expresses the vibration is the frequency of the mode, commonly expressed as the Raman shift in wavenumbers, $\bar{v}_{i}$, expressed by convention in $\mathrm{cm}^{-1}$. The Raman shift relates the energy difference between the incident photon (of energy $E_{L}$ ) and the scattered photon (of energy $\left.E_{S}\right)$ as:

$$
\Delta E_{R}=E_{L}-E_{S}=h c \bar{v}_{i}
$$

where $h$ is Planck's constant and $c$ is the speed of light. The wavenumber defines the energy lost (in a Stokes event) or gained (in an anti-Stokes event) by the photon 
due to interaction with the molecule.

\section{Raman cross-sections}

To quantify the efficiency of the Raman process we define the Raman crosssection, $\sigma\left[\mathrm{m}^{2}\right]$, as the proportionality constant relating incident to Raman scattered light. The cross-section in this case comes from an isotropically averaged version of the Raman tensor (as would occur in a liquid or gas phase).

$$
P=\sigma S_{0}
$$

where $\mathrm{P}[\mathrm{in} \mathrm{W}]$ is the intensity (proportional to number of photons per unit time) of the scattering events relative to the incident power density $S_{0}\left[\mathrm{~W} / \mathrm{m}^{2}\right]$ at the molecule position.

However, this form of the cross-section does not consider the directional profile of the scattering of a given molecule. In an experimental situation, it is almost always the case that we are detecting the scattered radiation in a particular direction (typically $90^{\circ}$ or back-scattering detection). To take this into account we define the absolute differential Raman cross-section $\left[\mathrm{in}^{2} / \mathrm{sr}\right], \frac{d \sigma_{\mathrm{RS}}}{d \Omega}$, as:

$$
\left\langle I_{\mathrm{RS}}^{\mathrm{SM}}\right\rangle=\frac{d \sigma_{\mathrm{RS}}}{d \Omega} S_{0} \delta \Omega
$$

where $S_{0}$ is the incident laser intensity $\left[\mathrm{W} / \mathrm{m}^{2}\right]$ at the molecule position and $\delta \Omega$ [sr] is the small solid angle for light collection, which in turn relates to the numerical aperture of the collecting optics. The Raman scattered intensity $\left\langle I_{\mathrm{RS}}^{\mathrm{SM}}\right\rangle[\mathrm{W}]$ is the signal obtained for a given vibrational mode of a single molecule, averaged over all orientations of the molecule in space $[6,7]$.

If we were able to detect the signal in all directions, we would obtain the total absolute Raman cross-section as:

$$
\sigma=\int \frac{d \sigma_{\mathrm{RS}}}{d \Omega}(\Omega) d \Omega
$$


Note that such measurements require specialized equipment such as an integrating sphere, which would involve a complicated and time-consuming measurement process. As a result, unless specified, when the term Raman cross-section is used in this thesis, and in much of the SERS literature, it is referring to the absolute differential Raman cross-section, $\frac{d \sigma_{\mathrm{RS}}}{d \Omega}$.

\section{Depolarization ratio}

If we begin to use polarized detection in our Raman experiment, we discover that the scattered light does not necessarily have the same polarization as the incident light. Thus, another important characteristic of a Raman mode is its depolarization ratio $\rho$, which is the ratio between the average Raman intensities for polarized detection perpendicular $\left(I_{\perp}\right)$ and parallel $\left(I_{\|}\right)$, respectively, to the incident polarization, $\rho=I_{\perp} / I_{\|}[6,7]$. In standard Raman spectroscopy, $\rho$ only depends on the symmetry of the mode (or more precisely on the symmetry of the Raman tensor) under consideration. For example, a perfectly isotropic mode would give a depolarization ratio of 0 . Uniaxial modes or those under resonance conditions normally result in a depolarization ratio of $\rho=1 / 3$. The depolarization ratio also relates the differential absolute Raman cross-section, $\frac{d \sigma_{\mathrm{RS}}}{d \Omega}$, to the total absolute Raman cross-section, $\sigma_{\mathrm{RS}}$, by:

$$
\sigma_{\mathrm{RS}}=\frac{8 \pi}{3} \frac{1+2 \rho}{1+\rho} \frac{d \sigma_{\mathrm{RS}}}{d \Omega} .
$$

Note that this can result in a difference of a factor up to $\sim 10$ for typical collection geometries, depending on the depolarization ratio of the mode.

\subsubsection{Semi-classical approach to Raman cross-sections}

We now move on to a more detailed description of Raman scattering based on the semi-classical description of Raman scattering [8], which treats the molecule quantum mechanically but considers the electromagnetic field classically. 
We will not be too concerned with elaborating the finer details or background of this approach, but rather present the following equations to show how one can obtain a Raman cross-section from the output of density functional theory (DFT) calculations. The ability to estimate the cross-sections of small, non-resonant molecules is a considerable aid throughout this thesis.

In this approach, the differential Stokes cross-section of a given vibrational mode with frequency $\omega_{i}$ (or wavenumber $\bar{v}_{i}$ ), excited by a laser at frequency $\omega_{L}$ (or wavenumber $\bar{v}_{L}$ ) can be expressed as: $[6,7,9]$

$$
\frac{d \sigma_{\mathrm{RS}}}{d \Omega}=C B_{i}^{2} R_{i} \bar{v}_{R}^{4} K(T)
$$

where $C=\pi^{2} /\left(45 \epsilon_{0}^{2}\right)$ is a constant $\left(\epsilon_{0} \equiv\right.$ permittivity of free space $=8.8542 \times$ $\left.10^{-12} \mathrm{Fm}^{-1}\right), \bar{v}_{R}=\bar{v}_{L}-\bar{v}_{i}\left[\mathrm{~m}^{-1}\right]$ is the wavenumber of the Stokes-shifted Raman signal, $B_{i}^{2}\left[\mathrm{~kg} \mathrm{~m}^{2}\right]$ is the square of the zero-point amplitude of the normal mode in reduced mass coordinates, and $R_{i}$ is the Raman scattering activity $\left[\epsilon_{0}^{2} \mathrm{~m}^{4} \mathrm{~kg}^{-1}\right]$. The factor $K(T)=\left(1-\exp \left(-h c \bar{v}_{i} / k T\right)\right)^{-1}$ accounts for thermal population of the vibrational state. Furthermore, $B_{i}^{2}$ can be obtained from:

$$
B_{i}^{2}=\frac{h}{8 \pi^{2} c \bar{v}_{i}} .
$$

Formally, the Raman Activity $R_{i}$ is a characteristic invariant upon arbitrary rotations of the Raman tensor of the probe. It can be expressed as:

$$
R_{i}=45 \bar{\alpha}_{i}^{\prime 2}+7 \bar{\gamma}_{i}^{\prime 2}
$$

where $\bar{\alpha}_{i}^{\prime}$ and $\bar{\gamma}_{i}^{\prime}$ are the isotropic and anisotropic invariants of the Raman tensor $\underline{\alpha}_{i}^{\prime}$ of the vibrational mode $[6,10]$.

An additional aspect that needs special attention is that Eqn. 2.11 is only valid for "isolated" (non-interacting) molecules in vacuo. If the sample under consideration is in the liquid state, at least two effects can arise: (1) the potential for interactions between molecules (either between the probe molecules or between the probe molecule and the solvent), and (2) the need to apply a local field correction due to the optical properties of the liquid. That is, the local field felt by a molecule is 
modified compared to the macroscopic field (of Maxwell's equations) created by the laser excitation. For Raman scattering, this leads to a local field correction of the cross-section in Eqn. 2.11 given by the factor [6, 11]:

$$
L=\left[\frac{\left(n^{2}+2\right)}{3}\right]^{4}
$$

where $n$ is the refractive index of the liquid (assumed here to be the same at both the excitation and Raman wavelengths, respectively; a good approximation for transparent liquids in the visible). This factor is usually not negligible (e.g. $L=2.5$ in water).

\subsubsection{Density functional theory (DFT)}

As mentioned, we utilize density functional theory (DFT) calculations as an aid to obtain absolute differential Raman cross-sections of small, non-resonant molecules. Here, we provide a brief theoretical background for these calculations. Interested readers are directed to Refs [12] and [13] for further details on DFT.

Essentially, DFT calculations seek to compute the electronic structure of a molecule, i.e. the wave-function of all its electrons for given atomic positions. At a fundamental level, the calculations start by solving Schrödinger's equations for a system of coupled electrons, but some of the most difficult parts to be dealt with are eventually introduced through a semi-empirical parametrization of the interaction in terms of the electron density. Thus it sits somewhere in between ab initio and semi-empirical methods.

On a practical level, there exist commercial (e.g. Gaussian [14]) and open-source packages (e.g. GAMESS [15]) that perform the DFT calculations and output the relevant values. The basic routine to perform a Raman vibrational analysis of a given molecule on such software is as follows [16]:

(i) The optimized molecular geometry (lowest energy) is obtained; and

(ii) The optimized atomic coordinates are then used as input for the evaluation 
of vibrational molecular properties, i.e. the Raman response of the molecule to an (oscillating) electric field.

In calculating the Raman response, the distortion of the electron density is calculated with respect to the unperturbed molecule for electric fields applied along the $x, y$ and $z$ axes. Combined with the normal mode displacement pattern this yields the associated change in polarizability, which can be reformulated as the Raman tensor. Specifically, the output gives us the Raman activity, IR activity, depolarization ratio, frequency, reduced mass, and force constant of the vibration. Using Eqns. 2.11 and 2.12, we can then obtain the differential Raman cross-section.

The 'black box' user of such packages should be reminded that these DFT calculations are not a perfect description of reality and, for example, tend to overestimate slightly the vibrational frequencies [17]. In many cases, the values can be effectively compensated by applying scaling factors. The DFT approach also ignores any time dependence, either from the vibrational oscillations or from the incident field of the laser. As such, any electronic absorption, excited state or resonance effect is entirely ignored [2]. The approach is therefore valid only for estimating the non-resonant Raman properties. Finally, there is a natural limit in terms of the computational time required to run the calculations. In practise, any molecule with more than about 15-20 atoms begins to take a long time (weeks and months) to run on a standard machine.

\subsection{Surface-Enhanced Raman Spectroscopy (SERS)}

So far we have presented some theory for one half of surface-enhanced Raman Spectroscopy (SERS), that dealing with Raman spectroscopy. In the following we discuss some background relating to the surface enhancement. In particular, we propose some suitable definitions for the enhancement factor (EF) in SERS, which we seek to accurately measure in later chapters. Again, the treatment is not intended to be greatly sophisticated but is given as a guide to understand the work that follows. 


\subsubsection{Discovery of SERS}

The SERS effect was discovered in 1974 by Fleischmann, Hendra and McQuillan (a NZ scientist) [18]. The group discovered an anomalously large enhancement of the Raman signal of pyridine in the presence of a roughened silver electrode. The enhancement was initially attributed to greater than expected, or fractal-like, surface area but subsequent reports by JeanMaire and Van Duyne [19] and Albrecht and Creighton [20] showed that the anomalous intensity could not be accounted for by increased surface area, and was in fact a new phenomenon, giving rise to the idea of the SERS cross-section.

Since then SERS has been extended to other metals (e.g. gold, copper, platinum, mercury, lithium, sodium, potassium, indium, aluminium, and rhodium) [21] and SERS, along with the closely related field of plasmonics, has flourished. The current day sees a plethora of acronyms related to SERS/plasmonics including Surface Enhanced Infrared Absorption (SEIRA), Tip Enhanced Raman Spectroscopy (TERS), Transmission Localized Surface Plasmon Resonance spectroscopy (TLSPR), Propagating Surface Plasmon Resonance spectroscopy (P-SPR), SurfaceEnhanced Flourescence (SEF), and Surface-Enhanced Hyper-Raman scattering (SEHRS), among many others [22].

Much of the impetus behind research in SERS is its great potential for applications. The enhancement in signal provided by the metal resolves the problem of the intrinsic inefficiency of the Raman process, and thus brings above the noise the rich spectral information available from vibrational spectroscopy. The combination of superb sensitivity and structural information make it an ideal detection platform for chemical sensing applications. An additional benefit of SERS is that fluorescence is quenched for molecules close to the surface, owing to the additional relaxation pathway available through transfer of energy to the metal surface.

However, while SERS has become a large and extremely active field of enquiry, there still remain basic questions as to the exact details of its mechanism and its magnitude. In general, two broad classes of enhancement mechanisms have been proposed, electromagnetic and chemical, with the electromagnetic enhancement 
playing the greater role and the chemical effect postulated to play a role for some analytes which are chemisorbed to the surface and form a complex with the surface metal atoms.

\subsubsection{Electromagnetic enhancement}

The electromagnetic enhancement can be viewed as essentially a redistribution of the EM field around the SERS substrate, resulting in strongly localised regions of high field intensities, so-called hot-spots. The effect is mediated through resonance of the light with the surface plasmons of the metal, with the enhancement falling off with distance from the substrate. Roughly speaking, plasmons are collective excitations of the surface conduction electrons that propagate along the metal surface. At certain frequencies of incident light these electrons become highly polarizable, giving rise to large EM fields. The details of plasmon resonance are complicated and a variety of terms exist to describe different aspects of plasmons depending on the research emphasis; such terms include surface plasmon polaritons, radiative and non-radiative plasmons, and localized and propagating plasmons [2]. Here we present only some of the most basic theory needed to outline the SERS effect. It should be noted that in the context of this thesis, all plasmon-related effects can be understood as electromagnetic effects, and the relation to the free electrons of the metal is only secondary. In this sense, all characteristics of plasmons is contained within the dielectric function (and its wavelength dependence) and the geometry of a specific problem.

In terms of the basic properties of the metal, it is suitable for use in SERS if its refractive index has:

(i) A negative real part of the dielectric function (preferably large and negative); and

(ii) A small imaginary part of the dielectric function.

The metals that fulfil this best are the alkali and noble metals $(\mathrm{Cu}, \mathrm{Ag}, \mathrm{Au}) . \mathrm{Ag}$ in particular is suitable for SERS applications in the visible and near IR because it 


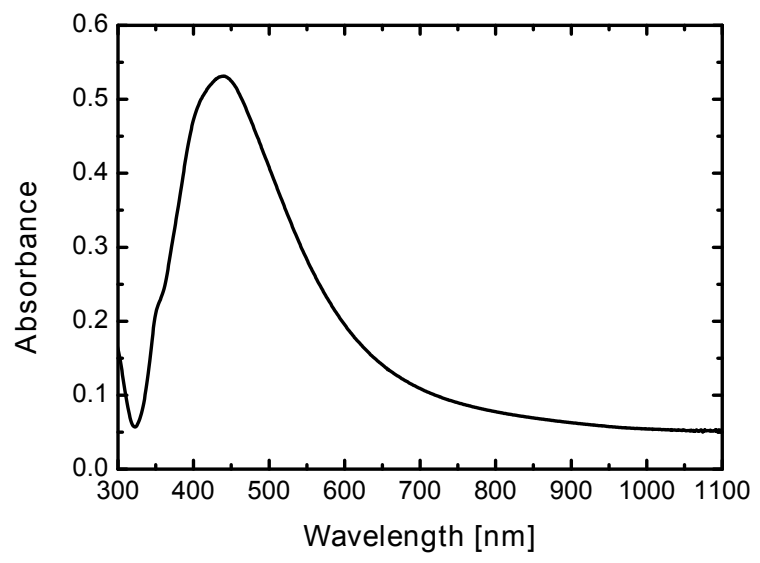

Figure 2.3: UV/Vis absorption spectra of a sample of the aqueous Lee \& Meisel colloids typically used in SERS experiments showing the considerable plasmon dispersion of the aggregated colloidal sample. As stated in the main text, this profile cannot simply be used as an indicator of the SERS enhancement.

has a very small imaginary component in this region and thus is less "lossy" than other metals.

\section{Plasmon resonances and wavelength dependence in SERS}

In simple terms, the plasmon resonances of a metal can be considered in terms of certain electromagnetic modes that exist for a material body described by a local dielectric function and a given geometry [2]. When the electromagnetic modes of a substrate are resonant with the incident (or radiative) light from an external source, enhancement occurs. Put another way, the optical response of the plasmon results in a redistribution of the energy around the object, such that some regions are locally enhanced. This can be seen in the absorption spectra of a SERS substrate as given in Fig. 2.3. It should be noted, however, that we can not simply use the absorption spectrum as an accurate indicator of the SERS enhancement of a given substrate at a given wavelength. First, the Raman effect involves enhancement of both the incident and radiative photons (at different energies), so the final enhancement depends on the convolution of these individual enhancements. In addition, for resonant molecules, pre-resonance effects may mean that SERS enhancements occur well before the resonances shown by the absorption profile. 


\section{The $\mathrm{E}^{4}$ approximation in SERS}

A common model to explore the EM enhancement is within the $\mathrm{E}^{4}$ approximation, which considers Raman scattering in the vicinity of a metallic surface [21].

In this model, we account for separate enhancement of the incident radiation and emitted radiation inherent in the two-photon Raman process by defining a local field intensity enhancement factor $M_{L o c}\left(\omega_{L}\right)$ and a directional radiation enhancement factor $M_{R a d}^{d}\left(\omega_{R}\right)$ (that directed towards a given detection position). The single-molecule enhancement factor (SMEF) in SERS is then given as:

$$
S M E F \approx M_{L o c}\left(\omega_{L}\right) M_{R a d}^{d}\left(\omega_{R}\right)
$$

$M_{L o c}\left(\omega_{L}\right)$ characterizes how much stronger the field intensity is with respect to the intensity if there were no metal object present, and can be determined by solving the electromagnetic problem under specific conditions with an incident field $\mathbf{E}_{I n c} . M_{\text {Rad }}^{d}\left(\omega_{R}\right)$ gives the enhancement experienced by the Raman emitting dipole, which is correspondingly at a different frequency from the incident laser.

Calculating $M_{R a d}^{d}\left(\omega_{R}\right)$ is a much more difficult prospect, however, and for simplicity it is generally assumed that $M_{\text {Rad }}^{d}\left(\omega_{R}\right) \approx M_{L o c}\left(\omega_{L}\right)$. This approximation can be justified formally and its range of validity specified [23]. From the definition of $M_{L o c}\left(\omega_{L}\right)$, this then gives the enhancement as:

$$
S M E F \approx M_{L o c}\left(\omega_{L}\right) M_{L o c}\left(\omega_{R}\right) \approx \frac{\left|\mathbf{E}_{L o c}\left(\omega_{L}\right)\right|^{2}}{\left|E_{I n c}\right|^{2}} \frac{\left|\mathbf{E}_{L o c}\left(\omega_{R}\right)\right|^{2}}{\left|E_{I n c}\right|^{2}}
$$

When taken at a zero-Stokes shift $\left(\omega_{L}=\omega_{R}\right)$, this yields the $|E|^{4}$ approximation as:

$$
\operatorname{SMEF}\left(\omega_{L}\right) \approx \frac{\left|\mathbf{E}_{L o c}\left(\omega_{L}\right)\right|^{4}}{\left|E_{I n c}\right|^{4}}
$$

It is important to note that this approach only approximates the radiation enhancement and does not account for polarization effects or surface selection rules in 
SERS [23].

\section{Theoretical methods for calculating EFs}

For simple objects, such as an individual sphere or spheroid, it is possible to obtain analytical solutions for the EF using Mie theory as a basis [24, 25]. Generalized Mie theory extends the solution to two closely spaced spheres [26].

In most practical cases, however, numerical approaches are the only option. These include methods such as the finite-element method (FEM) and the finite-difference time domain (FDTD) techniques, which solve Maxwell's equations numerically.

Commonly, the simulations are simplified based on the electrostatics approximation (ESA), which treats the electric field as an electrostatic field but still considers the metal in terms of its frequency-dependent complex dielectric functions. This approximation works well for objects much smaller than the wavelength of light (20-40 nm), but should be used with caution on much larger systems. Another common approximation is to reduce the problem to two dimensions (2D), which works for problems that are effectively $2 \mathrm{D}$ in nature due to symmetry. Otherwise the $2 \mathrm{D}$ solution should only be used as a qualitative guide for the 3D case [2]. Further, most numerical methods rely at some point on a discretization (meshing) of the objects under study into small cells. The characteristics of the EM fields inside each cell are then computed. As a result, the size and shape of the cell is one of the most important issues then for the accuracy of the overall result. These factors place limits on the accuracy of the overall results particularly at sizes which approach the cell size of the calculation, in which case the results are heavily dependent on the size and shape of the cell.

The results of such methods give us a theoretical calculation based on Maxwell's equations of the distribution of EFs around particular object geometries and are an invaluable tool in SERS and plasmonics particularly. The most important result in the context of this thesis is that maximum EFs up to $\sim 1 \times 10^{11}$, sometimes $10^{12}$ are obtained for the best cases $[2,27,28,29]$. Again, only practioners with experience in such simulations can give an idea of the validity of a given approach 


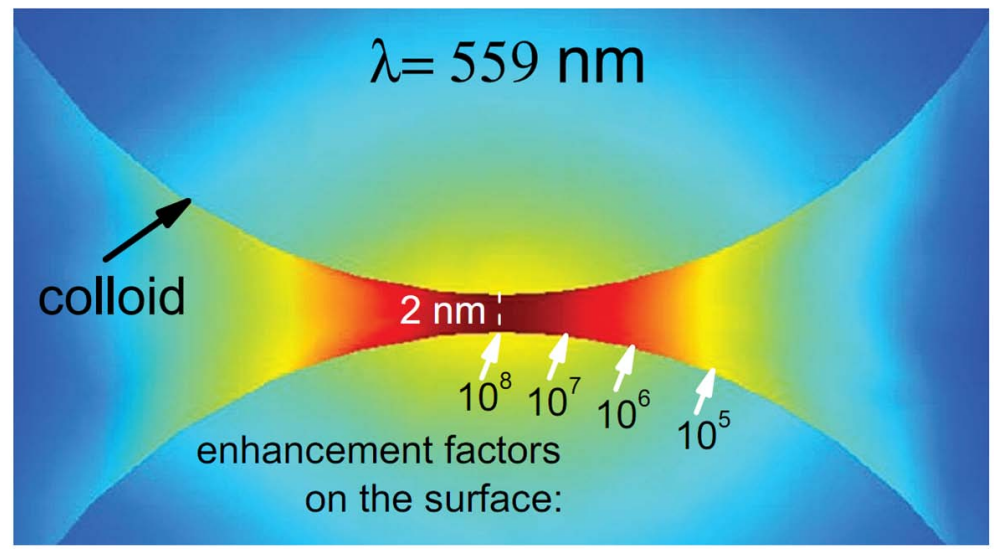

Figure 2.4: Pictorial of a hot-spot for a particle dimer showing the rapid change in EF even for a small change in position.

and thus of the final enhancement factor obtained. In this regard, it should be noted that the maximum theoretical enhancement factor used in this thesis is based on a general reading of the current literature, with direct confirmation from the analytical methods available such as Mie theory [24].

In addition, simulations confirm that the optimum enhancements occur: $(i)$ at the tip of objects with pointy curvatures, i.e. sharp features, and (ii) in the gaps between closely spaced particles (with maximum EFs often obtained for particles separated by a few nanometres). Further, in the vicinity of these regions of high enhancements, or hot-spots, the enhancement changes dramatically even within a short distance from the region of maximum intensity [30]. This means that in most SERS substrates, the enhancements that mainly contribute to the SERS signal arise from extremely localized regions throughout the sample (see Fig. 2.4).

In terms of distance dependence, Mie theory using the ESA gives a distance dependence $d$ for a simple sphere of radius $a$ as $1 /(a+d)^{12}$. In practise, EM enhancements typically extend to at least $\approx 10 \mathrm{~nm}$ from the surface $[2,22]$. Thus, the effect can operate on non-bound molecules and extends beyond a monolayer for many molecules, which is an important distinction to the postulated chemical enhancements given in the next section. 


\subsubsection{Chemical enhancement}

The other type of enhancement postulated to occur in SERS is chemical enhancement, which requires the probe to be chemically bound to the SERS substrate. Chemical effects have been proposed to explain changes in the relative intensities (and frequencies) in the vibrational modes of a molecule as compared with the normal Raman spectra, and to account for perceived discrepancies between the maximum EFs found experimentally and the maximum values from EM calculations. The proposed chemical mechanisms can be divided into two categories $[22,31]$ :

(i) Charge transfer states involving transitions from the Fermi level of the metal to an unoccupied orbital of the molecule (or vice versa); or

(ii) Formation of a surface complex involving the metal and the analyte, leading to a change in the properties of the molecule (such as the possibility of resonance Raman scattering).

A significant body of work has been dedicated to teasing out the details of chargetransfer processes and the change of properties resulting from analyte-surface complexes [32, 31, 33]. Many of these studies have been performed on electrochemical setups and explain variations in analyte properties such as relative intensities that may not be able to be explained through electromagnetic mechanisms.

However, it should be noted that in regard to the issue of chemical enhancement in SERS, there is considerable controversy $[2,34,35]$. Some of the effects attributed to chemical enhancement may actually be due to SERS surface selection rules, which would also play an important role in the case of a molecule bound in a fixed orientation to a surface [21]. Further, when examining some of the recent literature it may be concluded that some of the motivation for the discussion of chemical effects has been on the perceived need to "bridge the gap" between EFs obtained theoretically and those reported experimentally in SM studies [36]. As we show later, the massive EFs reported in such experimental studies were actually the result of inadequate definition and measurement of the EF. 
In any case, in typical SERS conditions, it is always going to be difficult to separate any chemical effects from possible contributions of EM origin, such as surface-selection rules or other mechanisms. Also chemical enhancement mechanisms exert a minor influence (up to $10^{2}$ in the best cases) [37] in comparison to the EM mechanism, and are by definition a general phenomenon. For particular analytes, however, chemical enhancements may play an important role, and such surface effects are a topic of study in their own right.

\subsubsection{A note on surface adsorption}

Throughout the thesis, there is sometimes a distinction made in discussion about whether molecules might be directly bound to the surface or are simply attracted to the surface but do not form strong chemical bonds, e.g. through Van der Waals attraction or electrostatic forces. The distinction can be framed in a number of ways, as in the use of chemisorption for the former case and physisorption for the latter, but it is important to clarify that there is no easy way to determine the degree of analyte-surface binding, and additionally, there is no clear boundary between the different processes involved in terms of our ability to distinguish them. For our purposes, we use the more general term"adsorption" throughout the thesis as we do not attempt to determine the mode of binding but rather are interested in whether or not there is evidence for a change in the molecular properties at the surface, and in particular, whether this change somehow leads to some additional enhancement for a specific analyte in certain cases, i.e. as in chemical enhancement.

\subsubsection{Other mechanisms}

Another possible enhancement in SERS, discussed very early after its discovery $[38,39]$ involves the interaction of a dipole self-reaction field with its polarizability. This self-reaction field then modifies the ability of the dipole to radiate energy, i.e. it can effectively oppose or amplify the dipole amplitude. This effect is effectively then defined as a 'chemical enhancement', given that it involves a change 
of polarizability upon adsorption; however, the origin is actually electromagnetic. We do not give an expression for the potential enhancement here, which can be approximated by an image dipole method, but include the effect to demonstrate the subtle distinction in this case between modification of the enhancement and the enhancement itself. It should be noted that none of the results in this thesis depend on the subtleties of these mechanisms, which we cannot distinguish in any case from our experimental data.

\subsection{Single-Molecule (SM) Detection}

\subsubsection{Background}

The potential to use SERS to detect substances to a level down to a single molecule has generated a lot of interest since enhancement factors of up to $10^{15}$ were reported by Kneipp [36] and separately by Nie and Emory [40] for the dye rhodamine $6 \mathrm{G}$.

Despite the obvious impact of these papers in terms of their effect in reinvigorating the SERS field, the evidence for SM detection presented at that time falls short of a definitive proof upon detailed consideration, and indeed has been seriously questioned in the literature [41, 42]. The original proof and other subsequent reports [36, 40] used aggregated colloidal solutions as substrates with ultra-low analyte concentrations (sub-picomolar) to achieve a nominal analyte concentration of less than one molecule per colloid, typically $\sim 0.1$ molecule/colloid. At such low concentrations, it was simply assumed that any observed signal is single molecule in character because of the scarcity of the probes in the sample. However, working at ultra-low concentrations is very difficult [43], and one cannot exclude the possibility that several molecules exist at the same place, as a dimer or larger aggregate for example.

Further proof of SM detection was presented by Kneipp et al. [36] in the form of the supposed observation of a Poisson distribution in the statistics of SERS intensities. That is, it was argued that quantized signals could be observed repre- 
senting one, two, or several molecules. However, a number of problems become apparent with this description on closer examination. Chiefly, the ability to detect quantization from a distribution of intensities based on a number of different spectral events assumes that molecules experience equivalent enhancements at different hot-spots in the sample. In fact, the colloidal solutions used in these experiments obey a long-tail distribution, with the enhancements varying considerably throughout the sample and in-and-around individual hot-spots [44]. This long-tail distribution means that it is essential to obtain sufficient statistics, otherwise an erroneous conclusion may be made about the distribution if only certain parts of the tail are sampled. In the case of Kneipp et al, later work [42] has shown that, in fact, the combination of a limited number of statistics $(\sim 100)$ fitted to a small number of bins ( $\sim 20)$ using a considerable number of parameters (12 free parameters) is responsible for the assumption of Poisson statistics.

A long-tail distribution would, in itself, quickly wash out any Poisson distribution. The relative lack of observable events over the experimental time in these cases highlights the difficulties of an ultra-low concentration approach. In a given aggregated solution only a small proportion of the available hot-spots give the necessary enhancement for detection of a single molecule. The convolution of the inhomogeneous enhancements with ultralow concentrations results in the observation of events that are extremely rare. The limited statistical reliability thus leaves room for alternative explanations such as the more selective adsorption of analytes by certain colloid particles, or the formation of dye aggregates [41].

Other evidence for SM detection has been based on spectral fluctuations and the 'blinking' phenomena $[40,45]$, whereby the signal is observed to rapidly fluctuate and switch on and off. Such blinking was observed in SM fluorescence $[46,47]$, where on-off spectral behaviour originates as a consequence of the electron of an individual molecule being trapped for a time in the triplet state, where fluorescence is not possible. Thus the on-off behaviour is a good indication of single molecule fluorescence (SMF) when careful experiments are performed. Several authors have concluded that the phenomenon is also indicative of SM SERS $[48,49,50]$, but the evidence is far from a definitive proof in this case. It should 
be noted that "blinking" in this way is actually impossible in Raman, because the Raman effect cannot be "turned off" as in fluorescence. As such, even the label "SERS blinking" if by itself misleading. In fact, other effects may well explain the observed spectral fluctuations, including photo-bleaching of the dyes, photodesorption, photo-induced surface diffusion, and substrate heating or morphology changes $[41,51]$. Because many of these effects are cooperative, i.e. they are likely to affect all molecules at the same time (for example when the metal reaches a critical temperature), they could, in principle contribute to the SERS fluctuations and to "blinking", even in many-molecule conditions. Such fluctuations are actually often observed, even in conditions of relatively high concentration, where the signal is not believed to originate from SMs.

As such, although the reported detection of single molecules reinvigorated the field and pointed the way towards the great potential of SERS as a trace detection technique, an unambiguous proof of SM detection had still not been established.

\subsubsection{Bi-analyte SERS (BiASERS)}

A possible solution to the SM problem came with the BiASERS technique developed here at Victoria University. The BiASERS method [41] works as a contrast method to allow one to observe the statistics of events of one dye against the background of signals produced by the other. The use of this method allows for the use of moderately low analyte concentrations (up to $100 \mathrm{nM}$ ), rather than ultralow concentrations, and consequently large numbers of statistics can be gathered in a much shorter time. Thus, the conclusion of SM detection is always based on the acquisition of sufficiently many statistics. Hence, the method provides an additional degree of freedom with respect to plain intensity fluctuations [42] to decide on the "SM character" of the signal.

A simple analogy for BiASERS is to imagine the hot-spots as separate "buckets" distributed randomly. We then randomly throw balls of two different colours (representing two spectrally distinct molecules) in the general direction of the buckets to the point that, eventually, some of the buckets are filled with no balls, some 
have a single ball, while some have several balls (i.e. a situation of moderate "concentration"). In the analysis stage, we start by identifying the buckets that have balls of only one colour. Taking simple statistical intuition as our guide, we can quickly see that the number of balls in these one-colour examples is going to be very low - a single ball, or perhaps two or three, but not very many. For buckets with many balls, the random distribution of the two balls would most commonly result in a mixture. This then is the BiASERS technique: by using two analytes, one as a contrast agent for the other, we are able to identify signals arising from one, two or a few molecules. The collection of a sufficiently large set of data then allows us to definitively prove the detection of SM events. More detailed analysis of the statistics of hot-spots with more realistic models has been presented in Ref. [52]. Note that the bucket analogy is only a rough guide to the experimental reality-for example, in a real SERS experiment the different buckets would be of different sizes and would give different enhancements.

Application of the BiASERS technique perhaps works best in liquid measurement of a solution of aggregated colloids, because in this case Brownian motion allows for the collection of a large set of independent data without difficulty-we simply repeat acquisition over a short time for a long duration. In practise, the two dyes should have similar properties (adsorption and Raman cross-section), and must of course have distinct SERS spectra to allow for separate identification.

The first example of SM detection by this method [41] has paved the way for much subsequent research based on multiple analyte techniques [41, 53, 54, 55, 56, 57] to identify, categorize, and classify SM events in SERS. In Chapter 5, it is the key method for obtaining an accurate estimation of SMEFs in SERS.

\subsection{The SERS Enhancement Factor}

As the thesis title suggests, the SERS enhancement factor (EF) is the central topic of the work to be presented. In the following, we set the necessary theoretical framework for understanding the EF problem, which will be extensively applied in Chapter 5. 
In its simplest expression, the EF is a value that compares the increased intensity in signal obtained under SERS conditions to that under normal Raman conditions (non-SERS).

However, due to the diversity of situations that can arise in SERS - single molecules, multiple molecules, experimental limitations (not knowing the exact number of molecules, for example, averages over time, varying spatial distribution, orientations of the probe on the surface, surface coverage, different substrates, etc.) obtaining a suitable definition is not straightforward. Our purpose in measuring the EF and the experimental conditions we are under will motivate our choice of definition.

We explore in the next sections some basic definitions that cover a range of typical SERS situations. The important thing here is that the definitions are unambiguous, although our ability to characterize some of the parameters may differ depending on the situation. Note that, while we present the EFs here in terms of intensity for simplicity, the alternative, which we shall explore later, is to characterize the Raman and SERS intensities in the form of cross-sections, and express the EF as a ratio of these cross-sections.

\subsubsection{The single-molecule enhancement factor (SMEF)}

The SMEF is the enhancement felt by a given molecule at a specific point. It is, in general, dependent on the Raman tensor of the probe and its orientation on the SERS substrate and with respect to the local field at that point. In turn, it also depends on the orientation of the SERS substrate with respect to the incident laser polarization and direction. Because of these constraints, a rigorous definition is more suited to theoretical estimations.

However, estimating the SMEF of an analyte on a given substrate is still instructive in an experimental setting, especially if we look at the maximum available EFs and are able to sample many SM events in our experiment (those originating from hot-spots). 
In this case, we define the SMEF as:

$$
\mathrm{SMEF}=\frac{I_{\mathrm{SERS}}^{\mathrm{SM}}}{\left\langle I_{\mathrm{RS}}^{\mathrm{SM}}\right\rangle},
$$

where $I_{\mathrm{SERS}}^{\mathrm{SM}}$ is the SERS intensity of the single molecule under consideration, while $\left\langle I_{\mathrm{RS}}^{\mathrm{SM}}\right\rangle$ is the average Raman intensity per molecule for the same probe. This definition takes into account the fact that, in our normal Raman experiment, we cannot measure a single molecule or know the orientation of the molecule because we necessarily measure many molecules to obtain our signal.

\subsubsection{The SERS substrate enhancement factor (SSEF)}

If we are more interested in the average enhancement over a given substrate rather than the maximum enhancement, we can define the SERS substrate enhancement factor (SSEF), which allows comparison between the average SERS enhancements across different substrates. At a first approximation, the SSEF is given as:

$$
\mathrm{EF}=\frac{I_{\mathrm{SERS}} / N_{\text {Surf }}}{I_{\mathrm{RS}} / N_{\mathrm{Vol}}}
$$

where $N_{\mathrm{Vol}}=c_{\mathrm{RS}} V$ is the average number of molecules in the scattering volume $(V)$ at concentration $c_{\mathrm{RS}}$ for the Raman (non SERS) measurement, and $N_{\text {Surf }}$ is the average number of adsorbed molecules in the scattering volume for the SERS experiments. This is the general EF definition that has been used by most authors in the past $[58,59,60,61]$ and is normally taken as representative of a substrate. However, this definition presents difficulties in terms of experiment, in particular, because of the difficulty in determining the number of molecules that directly contribute to the SERS intensity. Generally, only rough estimates are made assuming monolayer coverage and maximum packing densities based on the molecular size. If we have a well-defined substrate, we can consider a rigorous definition of $N_{\text {Surf }}$ and $N_{\mathrm{Vol}}$ and define the SSEF in terms of the experimentally measured signals, 
under SERS ( $\left.I_{S E R S}\right)$ and Raman conditions $\left(I_{R S}\right)$ as:

$$
\mathrm{SSEF}=\frac{I_{\mathrm{SERS}} /\left(\mu_{M} \mu_{S} A_{M}\right)}{I_{\mathrm{RS}} /\left(c_{\mathrm{RS}} H_{\mathrm{eff}}\right)},
$$

where $c_{\mathrm{RS}}$ is the concentration of the solution used for the non-SERS measurement, $H_{\text {eff }}$ is the effective height of the scattering volume (defined rigorously in Appendix A.6), $\mu_{M}\left[\mathrm{~m}^{-2}\right]$ is the surface density of the individual nano-structures producing the enhancement, and $\mu_{S}\left[\mathrm{~m}^{-2}\right]$ is the surface density of molecules on the metal.

However, due to the difficulty in accurately estimating $\mu_{M}$ and $\mu_{S}$, particularly for the colloidal aggregates used in this work, we will not refer much to the SSEF, but include it here for completeness.

\subsubsection{The analytical enhancement factor (AEF)}

The definitions introduced so far, the SMEF and SSEF, have attempted to emphasize the intrinsic characteristics of the substrate and are not always straightforward to relate to experimental results. For many applications, one is mostly concerned with the simple question of how much more signal can be expected from SERS as compared to normal Raman under given experimental conditions. To address this question, we introduce another definition of the SERS EF, which is fairly intuitive and particularly relevant for analytical chemistry applications. Let us consider an analyte solution with concentration $c_{\mathrm{RS}}$, which produces a Raman signal $I_{\mathrm{RS}}$ under non-SERS conditions. Under identical experimental conditions (laser wavelength, laser power, microscope objective or lenses, spectrometer, etc.), and for the same preparation conditions, the same analyte on a SERS substrate, with possibly different concentration $c_{\text {SERS }}$, now gives a SERS signal $I_{\text {SERS. The analytical }}$ enhancement factor (AEF) can then be defined as:

$$
\mathrm{AEF}=\frac{I_{\mathrm{SERS}} / c_{\mathrm{SERS}}}{I_{\mathrm{RS}} / c_{\mathrm{RS}}}
$$

This definition, although useful for specific practical applications, tends to depend strongly on many factors such as the adsorption properties and surface coverage (monolayer vs. multilayer) of the probe; $c_{\text {SERS }}$ does not characterize well the 
number of adsorbed molecules. In particular, for 2D planar substrates, the actual number of adsorbed molecules is strongly dependent on the sample preparation procedure (e.g. spin-coating, dipping, or drying). For this reason, it is not a good characterization of the SERS substrate itself, and cannot be used to easily compare the performances of different substrates. However, provided all experimental procedures are clearly stated and sub-monolayer coverage is ensured, the AEF represents a simple figure for the SERS EF, whose measurement is easily reproducible. From its definition, it is also clear that the AEF is particularly suited to the case of SERS active liquids, e.g. colloidal solutions.

\subsection{Vibrational Pumping in SERS}

In this section we describe an alternative method for determining the SERS crosssection using a technique known as vibrational pumping. We use this method to obtain the SMEF for some analytes in Chapter 5 as supporting evidence to SMEFs obtained directly using the BiASERS method.

The use of SERS vibrational pumping to determine SERS cross-sections was proposed more than 10 years ago [62], but has been the subject of much controversy $[63,64]$ because, among other things, of the difficulties of distinguishing experimentally between pumping and heating effects $[65,66,67]$. Recent developments $[68,69]$ have shown that these difficulties can be avoided by monitoring the temperature dependence of the anti-Stokes to Stokes ratio down to low temperatures (typically $\sim 77 \mathrm{~K}$ or less), in the technique known as temperature dependent vibrational pumping (TDVP), described in the following section.

\section{Temperature dependent vibrational pumping (TDVP)}

In a SERS measurement, the population, $n$, of a single vibrational level over time is governed by three mechanisms: (i) pumping of the vibrations by the laser itself, proportional to the laser intensity $I_{\mathrm{L}}$; (ii) thermal excitation of the vibrational level; and (iii) relaxation of the vibration, defined by the vibrational lifetime $\tau$, 
through all possible mechanisms, including intramolecular vibrational relaxation, and external relaxation processes. Taking weak pumping, where $n$ remains small, we can define the rate equation for $n$ for a given vibrational mode, $\omega_{v}$, as [69]:

$$
\frac{d n}{d t}=\frac{\sigma_{\mathrm{S}} I_{\mathrm{L}}}{\hbar \omega_{\mathrm{L}}}+\frac{\exp \left(-\hbar \omega_{v} / k_{\mathrm{B}} T\right)}{\tau}-\frac{n}{\tau}=0,
$$

where the first term corresponds to an increase in the vibrational population (dependent on the Stokes cross-section $\sigma_{\mathrm{S}}$ ) due to pumping, and the second term that due to thermal excitation. $I_{L}$ and $\omega_{L}$ are the power density $\left[\mathrm{W} / \mathrm{m}^{2}\right]$ and frequency [rad s${ }^{-1}$ ] of the laser and $k_{\mathrm{B}}$ is Boltzmann's constant.

When $I_{L} \neq 0$, i.e. under laser pumping, we can obtain the steady-state population $n$ as:

$$
n=\frac{\tau \sigma_{\mathrm{S}} I_{\mathrm{L}}}{\hbar \omega_{\mathrm{L}}}+e^{-\hbar \omega_{\mathrm{v}} / k_{\mathrm{B}} T}
$$

In a practical measurement, the Stokes signal is independent of $n$ and is given by $I_{S}=N \sigma_{S} I_{L}$, where $N$ is the number of molecules. The anti-Stokes signal is proportional to $n$, and is given by $I_{\mathrm{a} S}=n N \sigma_{\mathrm{a} S} I_{\mathrm{L}}$. If we express these as the anti-Stokes/Stokes ratio, $\rho$, we can obtain $[68,69]$ :

$$
\rho=I_{\mathrm{aS}} / I_{\mathrm{S}}=A\left[\frac{\tau \sigma_{\mathrm{S}} I_{\mathrm{L}}}{\hbar \omega_{\mathrm{L}}}+e^{-\hbar \omega_{\mathrm{v}} / k_{\mathrm{B}} T}\right]
$$

where $\mathrm{A}$ is the asymmetry factor between the anti-Stokes and Stokes cross-sections, $\mathrm{A}=\sigma_{\mathrm{a} S} / \sigma_{\mathrm{S}}$, due to the underlying frequency-dependent plasmon resonances [70].

Experimentally, if we monitor the anti-Stokes/Stokes ratio, $\rho$, as a function of temperature for a given mode, we can observe a crossover temperature at which the population of $n$ changes from being in a temperature dominated regime to that governed by pumping (see Fig. 2.5). The temperature at which this crossover occurs changes depending on the mode energy as a consequence of the rapid variation of the exponential term; the larger the Raman shift, the large the crossover temperature will be. 


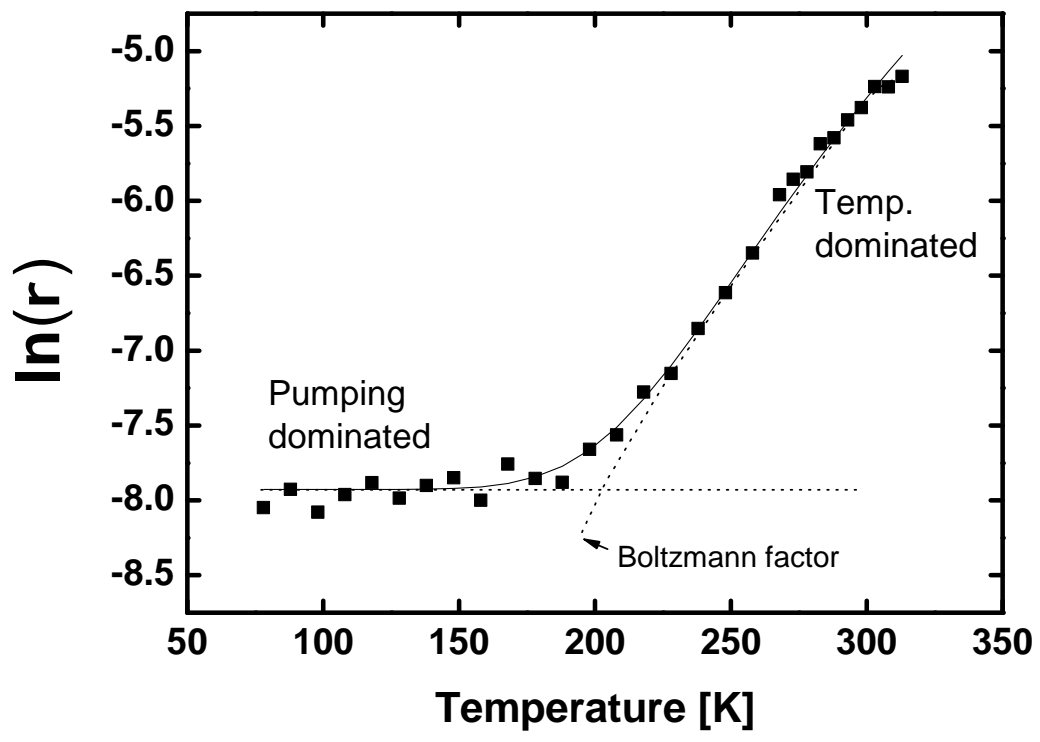

Figure 2.5: Example of fitting of the TDVP data of $\ln (\rho)$ with temperature for the $1510 \mathrm{~cm}^{-1}$ mode of rhodamine 6G. A crossover between the temperature-dominated regime and the pumping-dominated regime can be observed at $\sim 200 \mathrm{~K}$. In practise, the data are fit using Eqn. 2.25.

To provide the Stokes cross-section, we fit the data shown in Fig. 2.5 to a form of Eqn. 2.24 using:

$$
\ln (\rho)=a+\ln \left[b+\mathrm{e}^{-\hbar \omega_{\mathrm{v}} / k_{\mathrm{B}} T}\right]
$$

where $a=\ln (A)$ and $b=\tau \sigma_{S} I_{\mathrm{L}} / \hbar \omega_{\mathrm{L}}$. This is then a fit with two parameters, from which $\tau \sigma_{\mathrm{S}}$ can be obtained for a known $I_{\mathrm{L}}$. If we then estimate a value for $\tau$, we can obtain the cross-section $\sigma_{\mathrm{S}}$. Note that this method gives us an estimate for the asymmetry factor, A, which is effectively obtained as a proportionality constant for the curve in the high-temperature region. The asymmetry factor is not accessible by direct measurement of the Stokes and anti-Stokes signals because the anti-Stokes signal involves the convolution of anti-Stokes cross-section with the actual vibrational population for which the anti-Stokes process can occur. 


\section{Estimating the vibrational lifetime $\tau$}

To obtain the cross-section, then, we must estimate the vibrational lifetime of the mode. The $\tau$ values are estimated in our case by assuming the relaxation process leads to homogeneous peak broadening of the peak, as measured by the FWHM ( $\Gamma)$. In this case, $\tau$ follows directly from $\tau \sim \hbar / \Gamma$ [71]. In practical examples, it is found that the FWHM does not change by more than $1-1.5 \mathrm{~cm}^{-1}$ in peaks with a typical $\Gamma$ of $\sim 15-20 \mathrm{~cm}^{-1}$, suggesting an accuracy within $\sim 10-15 \%$ [68]. However, in addition to population relaxation, there are other contributions to the natural linewidths of the modes, including: (i) overlapping Raman peaks, (ii) inhomogeneous broadening, and (iii) phase decoherence, which may differ from mode to mode. Thus it is not straightforward to obtain an accurate value for $\tau$ for each mode.

However, if we can find a single peak in the spectrum where the population relaxation can be gained directly from the FWHM via $\tau \sim \hbar / \Gamma$, then all of the cross-sections of the other modes follow immediately by relative intensity of the peaks. That is, if we select a mode that is likely to be dominated by population relaxation, then we can use this mode to obtain the $\tau$ value and hence cross-section for this mode, along with all the other modes by relative peak intensity. The peak most likely to be dominated by population relaxation is usually the highest frequency mode, simply because of the greater number of vibrational states available to relax down to [69]. As a check of self-consistency, we then check that none of the $\tau$ 's are smaller than the value $\hbar / \Gamma$ estimated from their FWHMs. to ensure the consistency of the obtained $\sigma_{\mathrm{S}}$, the $\tau \mathrm{s}$ for each mode are checked to ensure that they are larger than the value of $\tau$ obtained from their widths $\Gamma$. This method, named the corrected lifetime method (CLM), must be judged on a case by case basis and, in particular, rests on the assumption that at least one of the modes has its broadening dominated by population relaxation. If this is not the case, all the cross-sections will be overestimated. However, using this method, we can obtain estimates for the SERS cross-section from the pumping experiment. The experimental details of TDVP are given in Section 3.4.4. 


\section{What cross-section are we measuring in pumping?}

In pumping, we are measuring the total SERS cross-section, $\sigma_{\text {Tot }}$, which can be greater than the differential radiative cross-section by a factor of up to $\sim 10$ depending on the Raman tensor of the mode. In addition, the pumping cross-section includes photons that would normally be absorbed by the substrate, and which would therefore not be observed in a direct SERS measurement. In this sense, the total cross-section is a combination of the radiative and non-radiative crosssections. These aspects have recently been studied in full detail in Ref. [72].

Naturally, the TDVP technique is indirect and can be influenced by a range of effects in the actual experiment including photobleaching and laser heating effects. However, if we are careful it gives us an estimate of the maximum available SERS cross-section and thus the maximum EF, an important value and one we can not always access by other approaches. 


\section{Chapter 3}

\section{EXPERIMENTAL}

We describe in this chapter the experimental details used throughout the thesis, including synthetic work, and preparation of the colloids and substrates used in SERS.

\subsection{Raman Setup}

Figure 3.1 shows the Raman microscope used in all Raman and SERS experiments and Fig. 3.2 gives a diagram of the beam path. The setup is a Jobin Yvon confocal Raman spectrometer coupled to a liquid $\mathrm{N}_{2}$-cooled CCD detector and a confocal (Olympus BX2) microscope in a back-scattering configuration. The internal laser is a $633 \mathrm{~nm} \mathrm{HeNe}$ laser and an $\mathrm{Ar}^{+}$-ion laser $(468,514 \mathrm{~nm})$ is used as an external source.

\section{Characteristic values for objectives}

In Table 3.1 we present the objectives used in this thesis along with characteristic values of the beam waist $\omega_{0}$ and scattering volume $V_{\text {eff }}$, which are used for calibrating the measurements in Chapter 5. These values were obtained using the beam profiling methods detailed in Appendix A.4 and are specific to the day of 


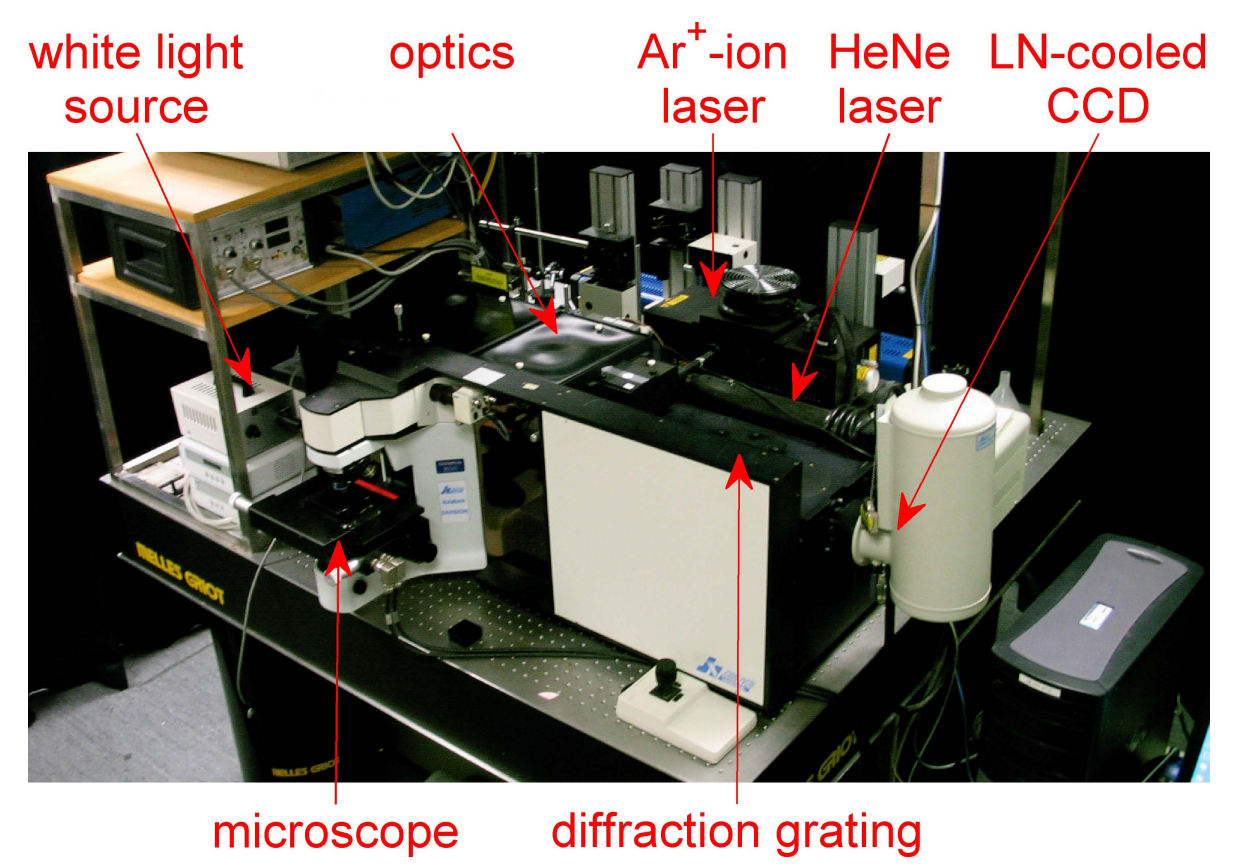

Figure 3.1: Raman microscope used for all Raman measurements in thesis.

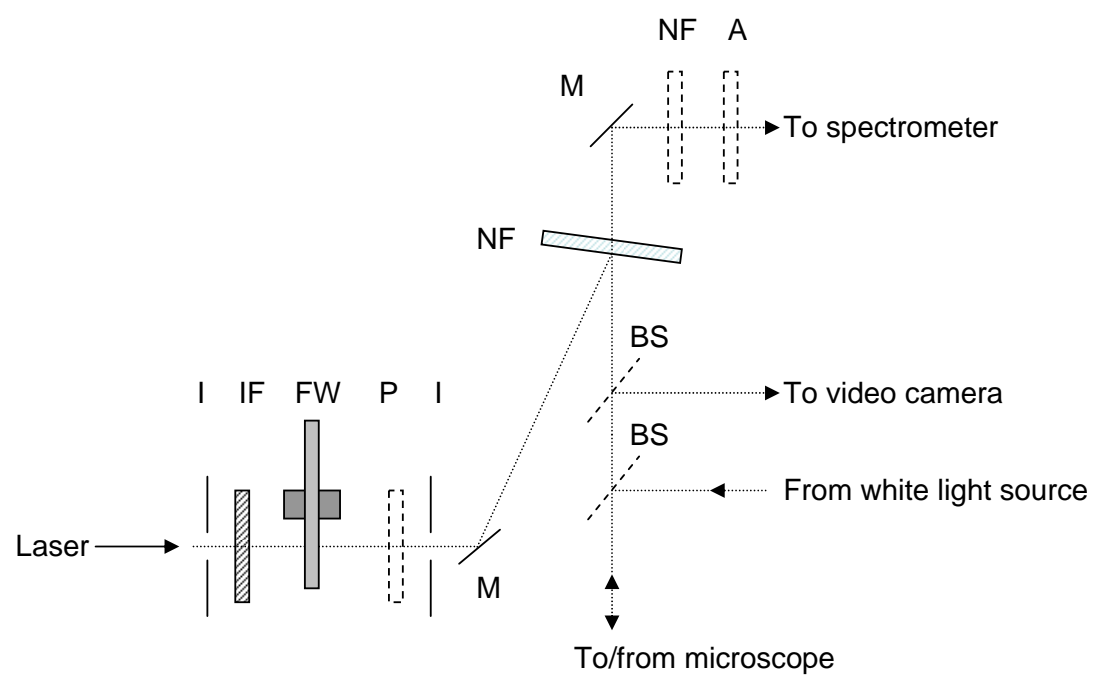

Figure 3.2: The beam path of the microscope in Fig. 3.1 as it enters the entrance slits of the monochromator. 
Table 3.1: Table of objectives used in this thesis with characteristic values measured by beam profiling experiments on the day of the experiment. The methods for obtaining the beam waist $\omega_{0}$ and scattering volume $\mathrm{V}_{\text {eff }}$ are described in detail in the appendices (see particularly Eqn. A.8). *The scattering volume of the $\times 10$ objective was not required.

\begin{tabular}{llccc}
\hline \hline Magnification & $\begin{array}{l}\text { type } \\
{[\mathrm{liq} / \mathrm{air}]}\end{array}$ & $\mathrm{NA}$ & $\begin{array}{c}\omega_{0} \\
{[\mu \mathrm{m}]}\end{array}$ & $\begin{array}{c}\mathrm{V}_{\text {eff }} \\
{\left[\mu \mathrm{m}^{3}\right]}\end{array}$ \\
\hline$\times 100$ & liq & 1.0 & 0.625 & 13.0 \\
$\times 100$ & air & 0.9 & 0.839 & 10.7 \\
$\times 50$ & air & 0.5 & 1.37 & 152.0 \\
$\times 10$ & air & 0.25 & 4.6 & N/A* \\
\hline \hline
\end{tabular}

experiments. For example, it can be expected that some variation will occur over time with different laser alignments. 


\subsection{Analytes used in Raman/SERS experiments}

All molecules used were sourced from Sigma-Aldrich except where otherwise stated and were used without further purification. Low concentration samples were prepared from stock solutions usually made up to $100 \mu \mathrm{M}$ in water unless solubility was a problem.

\subsection{Substrate Preparation}

The most essential factor in a SERS experiment is the substrate. Ideally, it should give the largest enhancements possible, be easy to prepare, and reproducible. In terms of giving the maximum EFs, aggregated colloidal solutions are among the best substrates available $[29,50]$. The EFs they produce, however, tend to be uncontrollable, which is the reason why all SM-SERS have to rely heavily on statistics. We detail here some common methods for obtaining and preparing such substrates.

\subsubsection{Synthesis of silver colloids}

\section{Synthesis of Lee \& Meisel colloids}

Using the method of Lee \& Meisel [73], $\mathrm{AgNO}_{3}\left(90 \mathrm{mg}, 2.12 \mathrm{mM}, 70 \mathrm{gmol}^{-1}\right.$ ) was added to distilled water $(500 \mathrm{~mL})$ in a $1 \mathrm{~L}$ glass beaker equipped with a stirring bar and the solution was brought to the boil. Upon reaching boiling, a solution of $1 \%$ tri-sodium citrate $\left(10 \mathrm{ml}, 100 \mathrm{mg}, 294.1 \mathrm{gmol}^{-1}\right)$ was added dropwise and the solution was kept boiling for $1 \mathrm{~h}$. The solution had a final $\mathrm{pH}$ of $8-9$. After cooling, the colloids were stored in plastic containers in a refrigerator. The colloids, depending on batch, were stable over 3-6 months. A reduction in $\mathrm{pH}$ was evident over the period (final $\mathrm{pH} \approx 6$ ).

The specific details of these colloids are covered elsewhere [74] but SEM measurements show that the colloids are widely polydisperse and have an average 


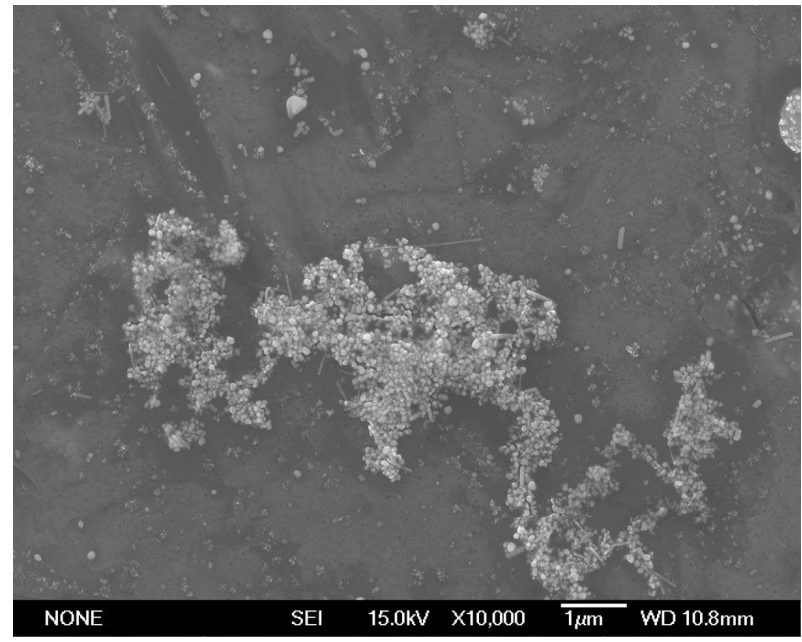

Figure 3.3: SEM image of Lee \& Meisel colloids showing an aggregated cluster from a dried sample of colloids deposited on silicon [74].

size of $60 \mathrm{~nm}$. UV-Vis absorption measurements show an extinction maximum at $\sim 400-430 \mathrm{~nm}$ with a long tail at longer wavelengths. They are a standard system in SERS due to the availability of large enhancements. Figure 3.3 shows an SEM micrograph of an aggregate of the Lee \& Meisel colloids.

\section{Syntheses of silver colloids using $\mathrm{BH}_{4}$}

\section{KCl method}

$\mathrm{KCl}$ (7.9 mg, $\left.74.6 \mathrm{gmol}^{-1}\right)$ was added to a solution of $\mathrm{AgNO}_{3}(18 \mathrm{mg}, 1.06 \mathrm{mM})$ in dist. water $(80 \mathrm{ml})$ in a $250 \mathrm{~mL}$ round bottomed flask equipped with a stir bar, with the resulting solution forming a cloudy blue precipitate of $\mathrm{AgCl}$. The solution was cooled on ice and an ice-cooled solution of $\mathrm{NaBH}_{4}\left(6 \mathrm{mg}, 37.8 \mathrm{gmol}^{-1}\right)$ in dist. water $(20 \mathrm{~mL})$ was added dropwise to the $\mathrm{AgCl}$ solution. Upon addition of the reductant, the solution became a cloudy yellow/grey colour. The reaction was allowed to warm to room temperature and was kept stirring under a fume hood for $1 \mathrm{hr}$ to react excess $\mathrm{NaBH}_{4}$. Depending on the preparation, the colloids were stable over $1-2$ months. 


\section{Albrecht and Creighton method}

Following the method of Albrecht and Creighton [75], solutions of $1 \mathrm{mM} \mathrm{AgNO}$ and $2 \mathrm{mM} \mathrm{NaBH}_{4}$ were prepared. To an ice-cold solution of $1 \mathrm{mM} \mathrm{AgNO} 3$ (2.5 $\mathrm{ml}$ ) in a round bottomed flask equipped with a stir bar, was added an ice-cold solution of $2 \mathrm{mM} \mathrm{NaBH}_{4}(7.5 \mathrm{ml})$ and the sealed flask was vigorously shaken. A yellow-grey solution resulted, which was stable over the short term $(2-4$ weeks).

\subsubsection{Preparing liquid colloidal aggregates}

\section{Individual SERS using aggregated Lee \& Meisel colloidal solution}

For most of the SERS measurements performed in this thesis, we used aggregated Lee \& Meisel colloid solutions aggregated by addition of $\mathrm{KCl}$. The particular proportion of $\mathrm{KCl}$ added has been optimized over several years of experiments on this colloid system; the long-term stability and characteristic clusters present in this system have been studied elsewhere [74]. Briefly, the amount of $\mathrm{KCl}$ added ensures that the colloids are aggregated close to (but below) the point of collapse. These substrates are semi-stable over hours and are known to give among the biggest EFs available in SERS.

To prepare the aggregated colloidal solution, the Lee \& Meisel colloids are mixed with a $20 \mathrm{mM} \mathrm{KCl}$ solution in a 1:1 proportion. The colloids are then left for a period of time (usually about $30 \mathrm{~min}$ ) before a small volume $(10-20 \mu \mathrm{L})$ of the probe molecule in solution is added to achieve the final desired concentration. At sufficiently low analyte concentrations $(\leq 100 \mathrm{nM})$, the stability of the colloids close to the aggregation limit is not affected by the additional screening of the repulsive potential introduced by the analytes. Depending on the particular batch and analyte used in the experiments, these colloid solutions may be active the next day although this can not be guaranteed. In this sense, the properties of the colloids are time dependent and so it is important to be consistent with timing when measuring them comparatively. Overall, the enhancements measured in these systems are expected to be similar as long as an identical process is followed (within 
$\sim 20 \%$ ) but there will inevitably be variation due to the original colloid preparation, which is sufficiently complicated and dependent on kinetic parameters (e.g. rate of reductant addition, water purity, exact source of starting materials) to be, as a general rule, only moderately reproducible. Further, the nature of the particular analyte may slightly affect colloid stability and the resulting enhancements even at the low concentrations used. The largest errors are thus systematic in nature and the enhancement factors quoted should be taken with this in mind.

\section{BiASERS using aggregated colloids}

In the basic BiASERS technique, two dyes with distinct Raman spectra are added at equal concentrations (usually from $5-50 \mathrm{nM}$ ) to an aggregated colloidal solution. A large number of spectra are then recorded (2000 or more) with short acquisition times (i.e. $0.05 \mathrm{sec}$ ).

\section{Concentrating SERS colloids using centrifugation}

In certain circumstances, it is desirable to increase the concentration of the colloids from that obtained by the synthesis. To do this, we centrifuge the colloids (from $5-15 \mathrm{krpm}$ ) until the colloids have settled in the container (but are not deposited on the walls), before removing a suitable amount of supernatant and reconstituting the sample using ultrasonic vibration.

\subsubsection{Preparing solid colloidal substrates}

In many cases, we use solid substrates prepared from dried colloidal aggregates as SERS substrates. A variety of different techniques for preparing such samples were explored in the course of the thesis. Here is a summary of the most important of these: 


\section{Drop cast colloids}

To ensure the analyte effectively "sticks" to the colloidal substrate, samples can be prepared by adding a drop of colloids onto a silicon wafer substrate and allowing the sample to completely dry under a lamp. With aqueous solutions, this inevitably results in a kind of "coffee-ring" structure, in which the greatest density of colloids lies at the outer edge of the drop, leaving the centre relatively sparse. Despite this drawback, samples can be quickly prepared and some reproducibility is possible as long as the region of interest (edge vs centre) is specified.

\section{Repeated deposition under blow dryer}

As an alternative to drying under a lamp, samples were prepared by forced evaporation with a heat gun on moderate heat, and instead of a single deposition the process was repeated several times to ensure effective coverage on the substrate. Briefly, a drop ( $50 \mu \mathrm{L}$ ) of concentrated colloidal solution (concentrated by $\sim 30$ fold) was pipetted onto a silicon wafer (drop area $\approx 5 \times 5 \mathrm{~mm}^{2}$ ) and a blow gun mounted on a clamp stand was directed at the sample from above. After the first drop had largely evaporated, another drop was added, with this process being repeated several times (usually $3-4$ times) to obtain the final substrate.

Before addition of the analyte, the as-prepared substrate was rinsed in dist. water to try to rinse off molecules attached to the metal colloids. For the SERS experiments, a drop of analyte was added and left to settle for $\approx 30$ mins in ambient conditions. Finally, the analyte drop was pipetted off, and the sample again rinsed in dist. water. Such a preparation proved suitable for preparing low concentration samples of non-resonant analytes. SERS was measured on these substrates close to the edge of the Si wafer, where the colloid surface coverage is generally larger as a result of the drying process. These substrates were used in Chapter 7 for detection of non-resonant probes, which were applied at concentrations of $25 \mathrm{~nm}$. 


\section{Polylysine-coated substrates}

An alternative to adhere the colloids to the substrate is to use an intermediate polymer layer such as polylysine, which as a positively charged polymer is able to attract the negatively charged citrate colloids. In such experiments, a low concentration (e.g. $0.005-0.01 \mathrm{wt} \%$ ) of polylysine (as poly-L-lysine hydrobromide) in aqueous solution is dried on a silicon substrate under a lamp. Subsequently, a drop of colloids containing the analyte added previously is placed on the substrate for a given period of time ( 5 mins), before siphoning off the drop and dabbing off the excess with a tissue. This forms a film of isolated colloidal aggregates, strongly attached to the substrate through electrostatic attraction, as poly-L-lysine is positively charged in solution.

\subsection{Supporting Experimental Techniques}

\subsubsection{UV-Vis spectroscopy}

UV-Vis spectra were performed on a double-beam Cary 100 instrument with mercury and tungsten sources in quartz cuvettes over the range $200-800 \mathrm{~nm}$.

\subsubsection{NMR}

For the synthetic work of Chapter $6,{ }^{1} \mathrm{H}$ and ${ }^{13} \mathrm{C}$ NMR spectroscopy (including COSY, HMBC, and HSQC experiments) were used to confirm the chemical structure and purity. The ${ }^{1} \mathrm{H}$ and ${ }^{13} \mathrm{C}$ NMR spectra were run on $300 \mathrm{MHz}$ and 500 $\mathrm{MHz}$ Varian spectrometers, respectively, in $\mathrm{CD}_{3} \mathrm{OD}$ as a solvent referencing to the residual methanol signal. The ${ }^{1} \mathrm{H}$ and ${ }^{13} \mathrm{C}$ NMR spectra are included in Appendix B. 


\subsubsection{Mass spectroscopy}

Mass spectra were acquired on a Q-Tof mass spectrometer with electrospray ionization. The samples were run in the positive $\mathrm{V}$ mode, and were calibrated by sodium formate for the mass range $0-1000 \mathrm{Da}$ (Q-Tof Premier, Waters Corp.). The mass spectra are included in Appendix B.

\subsubsection{TDVP measurements}

TDVP is performed by measuring the Stokes and anti-Stokes signals as a function of temperature down to $77 \mathrm{~K}$ using a nitrogen flow cryostat. The substrate is prepared by drop-cast deposition (see Section 3.3.3) of an aggregated Ag colloid sample prepared in a strongly aggregated state (as opposed to a partially aggregated state as for solution measurements), by using large analyte concentrations $(1 \mu \mathrm{M})$ [69]. In the experiment, a low power objective (i.e. $\times 10)$ that gives a large spot and low power density is preferred in order to: (i) reduce photobleaching, (ii) limit indirect laser heating, and (iii) improve sample averaging.

\subsubsection{DFT calculations}

For the DFT calculations, we used the Gaussian DFT package [14, 76] with Becke's 3-parameter hybrid functional [77] and Lee-Yang-Parr [78] non-local electron correlation (commonly abbreviated as B3LYP) with basis set 6-311++G(d,p). Using this package, we can carry out a vibrational frequency analysis and ultimately obtain the frequencies $v_{i}$, Raman activities $R_{i}$, and depolarization ratios $\rho_{i}$ for each peak, from which the gas-phase Raman cross-section at $633 \mathrm{~nm}$ can be calculated using Eqn. 2.11. The basic theory behind the process of DFT calculations is given in Section 2.1.6. 


\subsection{Synthesis and Characterization of Isotopic Dyes}

(i) Synthesis of 3,6-bis(ethylamino)-9- [2-(methoxycarbonyl)phenyl] xanthylium (Rh6M):

All chemicals were obtained from Sigma-Aldrich and used without further purification. Following a modification of the procedure of Abudara [79], phthalic anhydride $(0.20 \mathrm{~g} ; 1.35 \mathrm{mmol}, 99+\%)$ and $o$-ethylamino-p-cresol $(0.41 \mathrm{~g} ; 2.70 \mathrm{mmol}$, $95 \%$ ) were dissolved in the high boiling point solvent 1,2-dichlorobenzene $(5 \mathrm{~mL}$, b.p. $180.5^{\circ} \mathrm{C}$ ) in a flask equipped with a stirring bar, a reverse Dean-Stark apparatus and a condenser. After refluxing overnight, the reaction mixture was cooled to $50^{\circ} \mathrm{C}$, and sodium hydroxide $(36 \mathrm{mg} ; 0.9 \mathrm{mmol})$ and dimethylsulfate $(0.265 \mathrm{~mL}$; $2.8 \mathrm{mmol}$ ) were added. The resulting mixture was then heated at $90-100^{\circ} \mathrm{C}$ for 5 hours. In the workup, the reaction mixture was diluted with water $(8 \mathrm{~mL})$ and concentrated in vacuo (at $90^{\circ} \mathrm{C}$ ) to remove 1,2-dichlorobenzene by azeotropic distillation. This procedure was performed twice with water and once with methanol to ensure complete removal of the original solvent. The product was evaporated to dryness to give the product as a red residue ( $84 \%$ yield). Recrystallization from a mixture of concentrated hydrochloric acid $(1 \mathrm{~mL})$ and distilled water $(1 \mathrm{~mL})$ yielded metallic green needles.

Characterization of Rh6M:

The structure and purity were confirmed by mass spectroscopy and ${ }^{1} \mathrm{H}$ and ${ }^{13} \mathrm{C}$ NMR spectroscopy (including COSY, HMBC, and HSQC); see Sections 3.4.2, 3.4.3 for experimental details. ${ }^{1} \mathrm{H}$ NMR $\left(300 \mathrm{MHz}, \mathrm{CDCl}_{3}\right): \delta=1.41(\mathrm{t}, J=7.3$ $\mathrm{Hz}, 6 \mathrm{H}), 2.29$ (s, 6H), 3.55 (q, $J=7.3 \mathrm{~Hz}, 4 \mathrm{H}), 3.66(\mathrm{~s}, 3 \mathrm{H}), 6.69(\mathrm{~s}, 1 \mathrm{H}), 6.71$ $(\mathrm{s}, 1 \mathrm{H}), 7.28(\mathrm{dd}, J=7.4 \mathrm{~Hz}, J=1.5 \mathrm{~Hz}, 1 \mathrm{H}), 7.74(\mathrm{dt}, J=7.4 \mathrm{~Hz}, J=1.5 \mathrm{~Hz}$, $1 \mathrm{H}), 7.80(\mathrm{dt}, J=7.4 \mathrm{~Hz}, J=1.5 \mathrm{~Hz}, 1 \mathrm{H}), 8.31(\mathrm{dd}, J=7.4 \mathrm{~Hz}, J=1.5 \mathrm{~Hz}, 1 \mathrm{H})$ ppm. $\left.{ }^{13} \mathrm{C} \mathrm{NMR} \mathrm{(500} \mathrm{MHz,} \mathrm{CDCl}_{3}\right): \delta=165.4,157.0,156.6,134.4,133.0,131.3$, 130.2 , 129.8, 128.4, 126.3, 113.7, 94.2, 52.6, 38.7, 18.5, 13.9 ppm. HRMS [M]+ Calcd 429.2178; found 429.2174. UV-Vis $\left(\mathrm{H}_{2} \mathrm{O}\right): \lambda_{\max }=526 \mathrm{~nm}$.

(ii) Synthesis and characterization of 3,6-bis(ethylamino)-9-[2-(methoxycarbonyl)- d4-phenyl]xanthylium (d4-Rh6M): 
The deuterated version of Rh6M was synthesized according to the above procedure for Rh6M using instead d4-phthalic anhydride $(0.21 \mathrm{~g} ; 1.35 \mathrm{mmol}, 98 \mathrm{atom} \%$ D). Mass spectroscopy and NMR data again confirmed the target structure. ${ }^{1} \mathrm{H}$ NMR (300 MHz, $\left.\mathrm{CDCl}_{3}\right): \delta=1.40(\mathrm{t}, J=7.2 \mathrm{~Hz}, 6 \mathrm{H}), 2.28(\mathrm{~s}, 6 \mathrm{H}), 3.56(\mathrm{t}, J=$ $7.2 \mathrm{~Hz}, 4 \mathrm{H}), 3.66$ (s, 3H), $6.64(\mathrm{~s}, 1 \mathrm{H}), 6.70(\mathrm{~s}, 1 \mathrm{H}) \mathrm{ppm} .{ }^{13} \mathrm{C} \mathrm{NMR}(500 \mathrm{MHz}$, $\left.\mathrm{CDCl}_{3}\right): \delta=165.4,157.0,156.5,134.3,129.7,128.4,126.2,113.6,94.0,52.5$, 38.6, 18.5, 13.9 ppm. HRMS [M]+ Calcd 433.2429; found 433.2425. UV-Vis $\left(\mathrm{H}_{2} \mathrm{O}\right): \lambda_{\max }=526 \mathrm{~nm}$. 


\section{Chapter 4}

\section{ESTIMATION OF RAMAN CROSS-SECTIONS}

\subsection{Motivation}

This chapter and the following one form a central part of the thesis and outline our approach towards illuminating a central problem in SERS: the accurate quantification of the SERS enhancement factor (EF). Here, we provide some background to the EF problem, before outlining our particular approach. As we shall see, a key aspect of this approach is the consideration of the bare Raman (or non-SERS) cross-section, which the present chapter deals with. Chapter 5 then details the necessary steps towards obtaining the EFs for some common SERS probes.

\subsubsection{The EF problem}

The enormous "magnitude" of the enhancement in Surface Enhanced Raman Scattering (SERS) puzzled researchers right from its discovery. As described in the background (Section 2.2.1), the initial conflict of understanding in SERS was precisely around the "magnitude" of the effect and the difficulty in estimating the number of molecules that contributed to the signal. 
Since that time, the reported SERS cross-sections and corresponding EFs have been difficult to pin down [34]. The reliable estimation of EFs in different types of SERS active media therefore remains a longstanding ambition in the field of SERS.

An additional motivation for accurately characterizing SERS cross-sections and EFs is their importance in relation to the SM-SERS problem. Since the initial reports of SM-SERS spectroscopy over a decade ago [36, 40], there has been ongoing confusion as to the magnitude of the enhancement and its physical origin, along with uncertainties as to the reality of SM detection itself.

Finally, the SERS EF is a critical parameter in terms of characterizing the SERS effect for practical applications. In applications, the first concern is usually to know the magnitude of the EF that can be achieved for a given substrate and system. However, when scanning the literature on the design of new substrates, it is readily apparent that a consistent quantitative approach is lacking overall. In many cases, little attempt has been made to quantify the enhancement of a given substrate, despite claims of "high" or "large" enhancements. This makes comparison between different substrates and systems difficult, and the reader must make a best guess at the relative merits of a substrate from experimental details such as the laser power, integration time, type of objective, and Raman setupwhen this information is not made available, a guess is not even possible.

As some authors have pointed out [34], a significant criticism of SERS is that it has not lived up to original expectations due to the general failure in quantifying the signals accurately, which is linked to the difficulty in measuring the EFs adequately. In summary, a rigorous approach to quantifying the SERS EF ought to resolve longstanding contradictions and inconsistencies in the literature and is necessary for the establishment of SERS as a practical analytical tool.

\subsubsection{Previous measurements of EFs}

Several studies have concentrated on rigorously measuring the SERS EFs under specific conditions [58, 60, 61, 80], mostly focusing on average EFs. Average 
SERS EFs of the order of $\sim 10^{4}-10^{6}$ have been obtained, and sometimes as high as $\sim 10^{8}$ (depending on the definition and measurement procedure). However, anything from $10^{4}$ to $10^{14}$ may be quoted in the literature as SERS enhancements, in particular in relation to (but not limited to) the SM-SERS problem. It is a fact that the EF can strongly depend on the exact SERS conditions: substrate, analyte, excitation wavelength, etc. However, the wide discrepancy in the quoted EFs also arises from both a wide variability in the definition of the EF, and the way it is estimated in practise. In particular, inconsistencies in the reported values have arisen for the following reasons:

(i) Insufficient care in defining the EF;

(ii) Failure to normalize the SERS EF to the bare Raman signal of the probe; and

(iii) The use of basic estimates for the scattering volume and number of molecules adsorbed.

For example, in Ref. [36], the SERS EF for SM detection is estimated to be around $\sim 10^{14}$ based on a SERS measurement of the dye crystal violet (CV) using as a normalizing factor the Raman cross-section of methanol under the same experimental conditions. It is well known that dyes such as CV can have Raman cross-sections up to $10^{4}$ times larger than smaller molecules, especially in Resonance Raman Scattering (RRS) conditions [22, 81]. What is often less appreciated, and what we shall demonstrate later, is that such dyes still have large Raman cross-sections even in non-resonance conditions due to pre-resonance effects. This means that one cannot "guess" the non-SERS cross-section and then use it to estimate the SERS EFs. Such an approach is one of the causes for the apparently enormous EFs of $\sim 10^{14}$ frequently quoted in the literature. Therefore, the non-SERS cross-section must either be calculated from first-principles, such as in Ref. [81], or if possible measured experimentally (if the Raman signals are not completely engulfed by fluorescence). 


\subsubsection{Our approach}

In the work that follows, we seek to address these above points and obtain, we believe, the best possible estimates for the EFs of some common SERS analytes. The main features of this approach are:

(i) Careful definition of the SERS EF taking into account the specific optical setup and conditions;

(ii) Correct normalization of the EFs to the experimentally measured bare Raman cross-section;

(iii) Measurement of the SERS and Raman cross-sections with respect to a reference standard of known cross-section; and

(iv) Careful characterization of the scattering volume.

The EF definitions are considered in detail in the following chapter. In the present chapter, we look at how to properly normalize the EFs by including the bare Raman (non-SERS) signal, which is essential when estimating SERS EFs, either theoretically or experimentally.

\subsubsection{Why measure the actual Raman cross-section?}

As the basic EF definitions given in the background show (Section 2.4), it is usually not necessary to know the absolute cross-section of the analyte to measure the EF; a measurement of relative intensities can be enough.

However, knowing the absolute Raman cross-section is of great interest for several reasons:

(i) It highlights the fact that many SERS probes are in fact already very good Raman (non-SERS) scatterers and that different analytes can have widely different intrinsic Raman cross-sections; 
(ii) It is necessary if one wishes to determine the absolute SERS cross section from the SERS EF (for example, in order to be able to compare the effect with fluorescence);

(iii) In temperature-dependent vibrational pumping experiments where the absolute SERS cross-section is measured, the absolute Raman cross-section is required to determine the SERS EF; and

(iv) It avoids having to make non-SERS measurements, which usually involve high concentration samples and long integration times, each time a SERS EF is sought.

The last point is by no means trivial. It is often necessary to integrate for a long time (tens of minutes) to get a sufficient signal above the noise for bare Raman (non-SERS) measurements. Obtaining a characteristic value (the cross-section) for the molecule means the Raman signal only needs to be measured once-from then on we just need to compare the Raman cross-section to the effective SERS cross-section to obtain the EF.

A key aspect of our approach is using a reference of known cross-section to determine the Raman (and later SERS) cross-section. This process is described in the following section. 


\subsection{Measurement Methodology}

\subsubsection{Use of a reference}

The direct measurement of an absolute Raman cross-section is a time-consuming process, which requires the use of specialist equipment such as an integrating sphere. As an alternative approach, we measure the Raman cross-section of a given molecule relative to a reference standard with a previously reported crosssection. By ensuring the same optical conditions when measuring the reference and our unknown sample, we can obtain the unknown cross-section from the reference value by simple proportionality of the signals (taking into account concentration differences).

In practise, this means measuring the Raman signal of our analyte of interest followed as soon as possible by the reference. We then compare the intensities of the Raman peaks of our analyte of interest relative to a specific vibrational mode of the reference compound. Using the known cross-section of the reference (Ref), we obtain the Raman cross-section of the unknown sample (Sample) as:

$$
\left(\frac{d \sigma_{\mathrm{RS}}}{d \Omega}\right)_{\text {Sample }}=\left(\frac{d \sigma_{\mathrm{RS}}}{d \Omega}\right)_{\text {Ref }} \cdot \frac{I_{\text {Sample }}}{I_{\text {Ref }}} \cdot \frac{c_{\text {Ref }}}{c_{\text {Sample }}},
$$

where $c$ and $I$ are the concentrations and measured integrated peak intensities of the unknown sample (Sample) and reference (Ref), respectively. This equation refers to the case of liquid measurement using the same objective under identical conditions on the day of the experiment (i.e. the same laser power, alignment and hence scattering volume). We later use this approach to calibrate our SERS signal to obtain an effective SERS cross-section. The exact meaning of this SERS crosssection is specific to the EF definition and is discussed later.

Our basic approach, then, to measuring the Raman and SERS cross-sections (and thus the EF) is shown in Fig. 4.1. Our first task in this process is to find a suitable reference. 


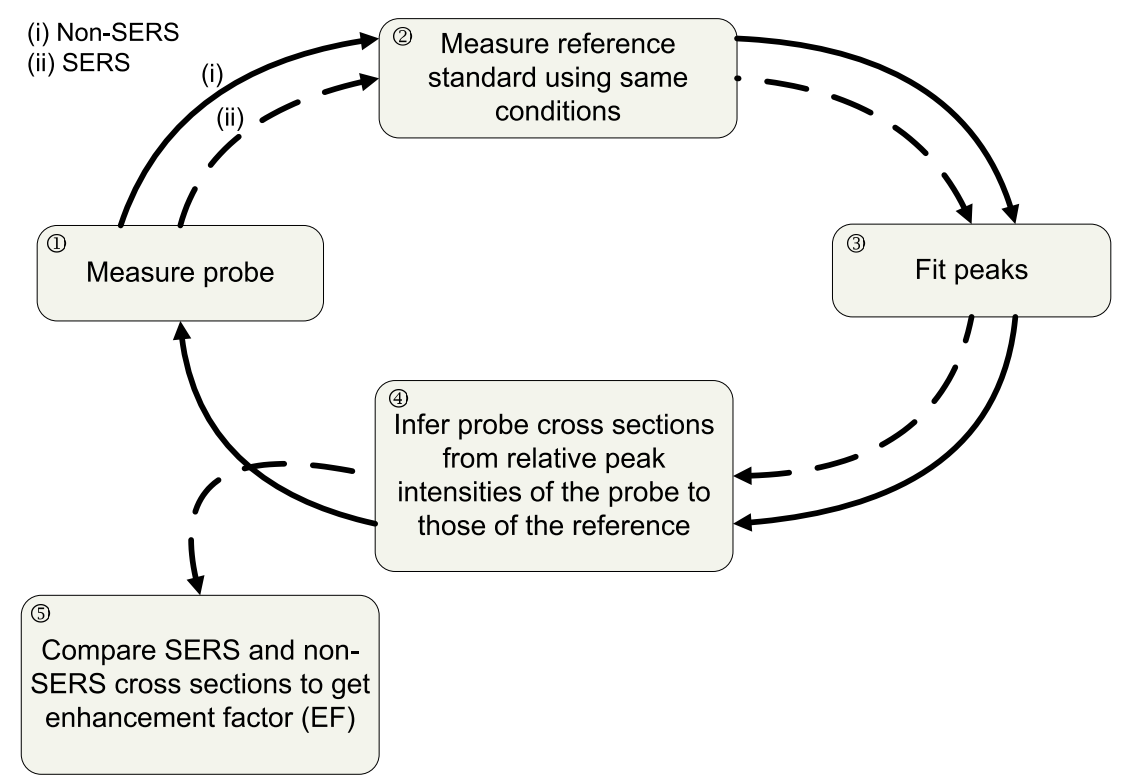

Figure 4.1: Method to obtain EF by measurement of (i) Raman and (ii) SERS crosssections with respect to a reference of known cross-section.

\subsubsection{Selection of a reference}

\section{Use of DFT as a validation tool}

In terms of the desirable properties of a reference, as well as having a known crosssection, it is also desirable that the compound is readily available, non toxic, and not too volatile.

It is critical also that we have confidence in the reported Raman cross-section of our reference compound taken from the literature. To validate the reported value, we make extensive use of Density Functional Theory (DFT) calculations to obtain absolute Raman cross-sections. To date, DFT has mostly been used in SERS to predict vibrational mode energies and assign them to their respective normal modes [82, 83, 84]. Here, we demonstrate its ability to predict the absolute Raman cross-section of simple non-resonant compounds, thus providing an additional layer of confidence in the reported value [6]. The derivation of the Raman crosssection from the DFT output is based on semi-classical theory [8] and is described 
in detail in Section 2.1.5. If the DFT value is consistent with the reported crosssection we can have an additional level of confidence in the reported value. Thus, the important value is still the original experimental Raman cross-section from the literature. While it is expected that DFT estimations for small, non-resonant molecules may give useful results $[82,83]$, this can not be assumed, and this was the reason we sought an experimentally measured value for the cross-section.

To further support the DFT calculation, we also measure some basic physical parameters of the compound experimentally, including the peak frequencies, relative peak intensities and depolarization ratios of the available modes, which then allows us to compare these values with the same parameters calculated by DFT. If the experimental and DFT parameters show good agreement, we have additional confidence in the DFT results, and consequently, the estimated and literature Raman cross-sections.

Note that since DFT is used here as an additional validation procedure, its exact implementation (basis set, etc.) is not crucial and must be ultimately justified by its agreement with experimental results. DFT predictions of Raman cross-sections cannot be trusted unless they are confirmed experimentally. This is because it is difficult to tell a priori whether resonant or pre-resonant contributions exist, which would then invalidate the semi-classical approach and therefore the DFT result; much more involved calculations, such as time-dependent DFT, are then necessary [81]. Also, the DFT calculations consider the molecule in the gas phase and do not consider the interactions that occur between molecules such as hydrogen bonding, solvent interactions, etc., and so in some cases the calculated frequencies and intensities of a molecule can diverge from those observed in experiment (see Section 2.1.6).

However, the comparison of DFT predictions with experiments is still instructive since when they agree: $(i)$ it indicates that there are no contributions from resonance/pre-resonance effects, and (ii) it confirms independently the experimentally measured cross-section. 


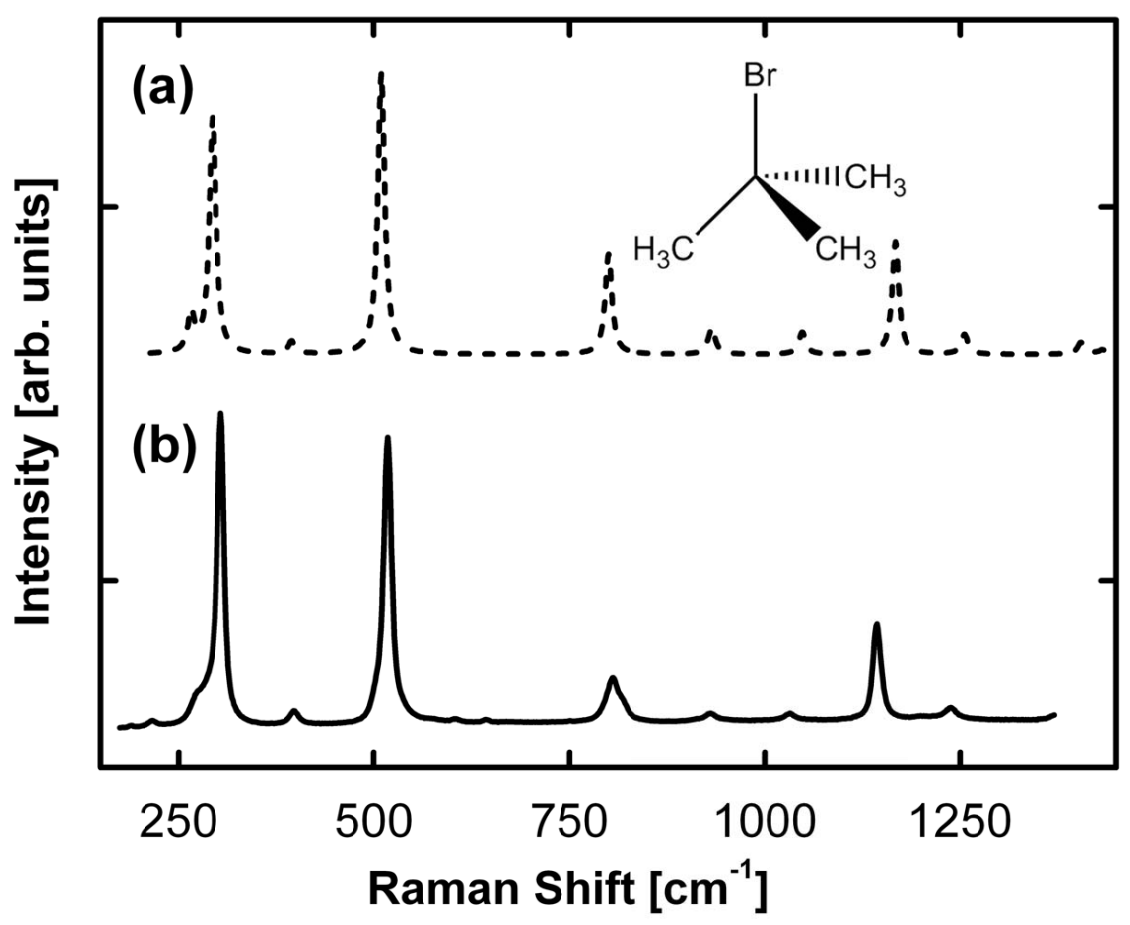

Figure 4.2: Comparison of Raman (non-SERS) spectra of 2-bromo-2-methylpropane (2B2MP) from (a) DFT calculations and (b) experiment. DFT calculations are shown without any frequency correction. There is excellent agreement between the DFT calculated and experimental spectra and cross-sections, providing good support for the use of this compound as a reference (for actual values, see Table 4.1).

\section{Screening for a suitable reference compound}

Several compounds screened initially showed significant divergence between the DFT predictions and experimental measurements (e.g. methanol, ethanol). This was assumed to be due to additional interactions likely to occur for these molecules such as hydrogen bonding, which is well known to affect the DFT estimates of alcohols [85]. Other compounds were unsuitable for practical reasons (e.g. dichloromethane was too volatile to be measured conveniently, and is nonetheless toxic). Eventually, we came across the simple solvent 2-bromo-2-methylpropane (2B2MP), which gave good agreement between the DFT measured cross-section and the reported value [6]. 
Figure 4.2 shows an experimentally measured spectra of 2B2MP along with a simulated spectra based on the Raman cross-sections calculated by DFT. For small molecules like this one, the predicted peak frequencies are in good agreement with experiments, thus allowing easy matching of the peaks between DFT predictions and experiments. In Table 4.1 we give the DFT values as well as the experimentally measured peak wavenumbers $\bar{v}_{i}$, relative intensities $I_{i}^{\mathrm{Rel}}$, and depolarization ratios $\rho_{i}$. As can be seen, the agreement between the experimental and DFT values is extremely good. The depolarization ratios $\rho_{i}$, for example, are within about $10 \%$ for most modes, which is excellent, and similarly, the relative peak intensities, $I_{i}^{\mathrm{Rel}}$, are consistent throughout most modes. The differences in peak frequency are within about $5 \%$ which is extremely good, given that a consistent overestimation of frequency is known for DFT calculations $[17,86]$.

The general agreement between DFT predictions and experiment indicates the DFT values are reliable for this compound and implies that the predicted absolute Raman cross-section should also be valid. Further, the Raman cross-section predicted by DFT is in excellent agreement with a previously reported value [6] (see Table 4.1). Finally, 2B2MP has the practical advantages that it is not too volatile and is non-toxic (although care must still be taken since it is flammable).

Both the reported and DFT cross-sections give us values in the gas phase, and so to use the reference in its liquid phase, it is necessary to deduce the liquid-phase absolute Raman cross-section of 2B2MP by using a local field correction factor of $L=3.3$, as described in Section 2.1.5 (Eqn. 2.14).

Based on the above results, 2B2MP was selected as a standard to determine the cross-sections of other liquids, using as a reference the $516 \mathrm{~cm}^{-1}$ peak with a reference absolute differential cross-section of $d \sigma / d \Omega=5.4 \times 10^{-30} \mathrm{~cm}^{2} / \mathrm{sr}$ at $633 \mathrm{~nm}$ in the liquid phase. 


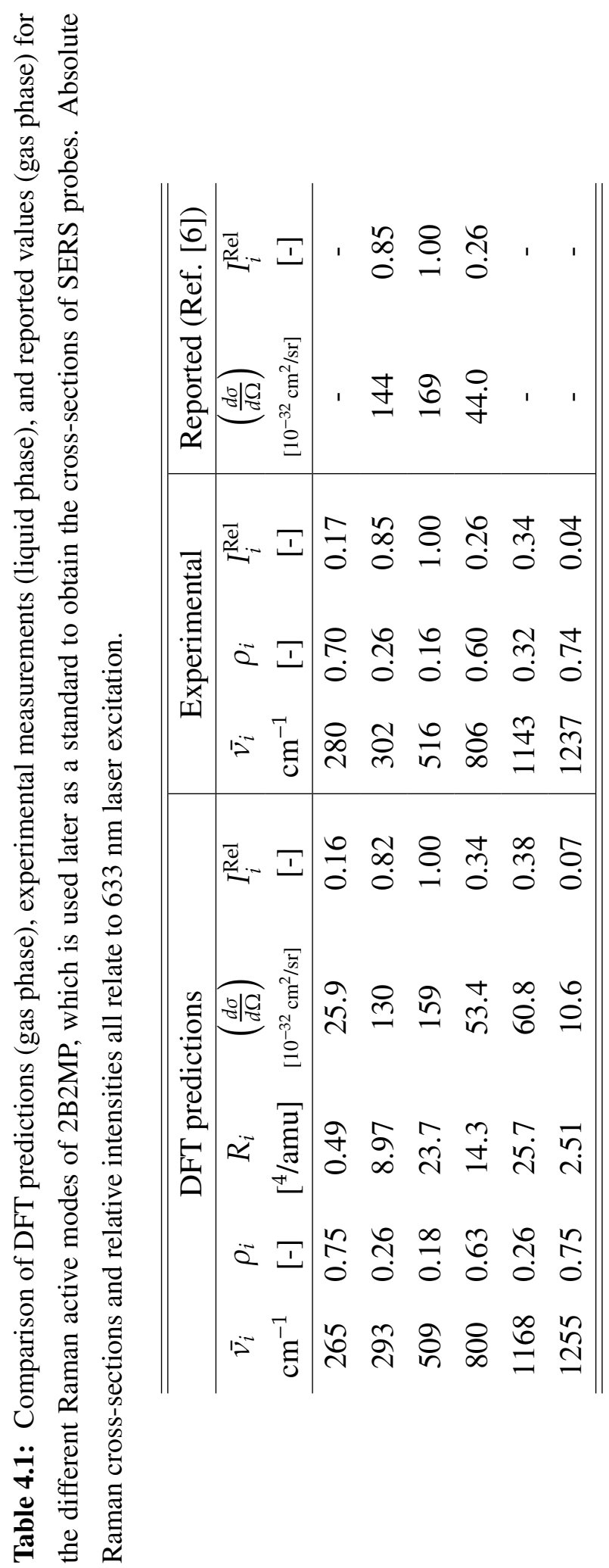




\subsubsection{Validation of reference using small molecules}

As an additional validation step, and as an intermediate case before estimating the cross-sections of some common dyes, we used the 2B2MP reference to estimate the non-SERS absolute cross-sections of several small, non-resonant liquids having Raman cross-sections reported in the literature. Again, we measure the sample and reference in quick succession to ensure identical optical conditions.

The results for the liquid compounds are shown in Table 4.2 along with previously reported cross-section values $[6,87]$. In this table, we also include the DFT values calculated for each of these compounds to further illustrate the predictive power of DFT in terms of absolute Raman cross-sections. However, the key comparison we are interested in is between the experimentally obtained value, estimated using the reference 2B2MP, and the previously reported values (corrected for the liquid phase). Again the data shows general agreement between the measured and reported values, thus confirming the validity of the 2B2MP reference and our approach in general. As a secondary observation, it is interesting to note that the DFT cross-section is also consistent with the measured and reported values. The discrepancy between DFT and experiments for dichloromethane is attributed to strong intermolecular interactions in this case.

The fact that our measurements are either above (benzene) or below (toluene) reported values indicates that our reference is a good compromise amongst the existing standards. It also highlights the experimental difficulties in accurately measuring absolute cross-sections and the unavoidable uncertainties in the reported values in the literature. For these reasons, we believe that the DFT predictions may sometimes provide an additional level of confidence in a given standard. 
Table 4.2: Comparison of the absolute Raman cross-section of common liquid reference compounds obtained from: (meas.) our measurements using the $516 \mathrm{~cm}^{-1}$ mode of 2B2MP as a reference; (rep.) reported values [6, 87]; and (DFT) obtained from DFT calculations with a local field correction factor as defined in Eqn. 2.14.

\begin{tabular}{l|c|ccc}
\hline \hline & $\begin{array}{c}\bar{v}_{i} \\
{\left[\mathrm{~cm}^{-1}\right]} \\
\text { meas. }\end{array}$ & \multicolumn{3}{|c}{$\left(\frac{d \sigma}{d \Omega}\right)$} \\
{$\left[10^{-30} \mathrm{~cm}^{2} \mathrm{sr}^{-1}\right]$} \\
meas. & rep. & DFT \\
\hline Benzene & 992 & 8.3 & 7.9 & 8.8 \\
\hline Toluene & 1002 & 2.9 & 3.5 & 3.8 \\
\hline Dichloro- & 282 & 1.7 & 1.9 & 3.1 \\
methane & 713 & 2.9 & 3.1 & 2.3 \\
\hline \hline
\end{tabular}

\subsection{Raman Measurement of SERS Probes}

Having validated the reference and our methodology for obtaining absolute Raman cross-sections for liquids, we then turned our attention to the original goal of measuring the Raman cross-sections of some common SERS probes.

\subsubsection{Analytes of interest}

The analytes that we selected for measurement are five commonly used SERS probes (see Table 4.3), which are expected to have quite different cross-sections at $633 \mathrm{~nm}$ laser excitation, namely:

(i) Crystal violet (CV), a dye that absorbs strongly with a maximum at $590 \mathrm{~nm}$ and is, accordingly, close to a Resonance Raman Scattering (RRS) condition;

(ii) Rhodamine 6G (Rh6G), another dye, which has a maximum at $528 \mathrm{~nm}$ and could therefore a priori be considered as non-resonant; 
(iii) Rhodamine 123 (Rh123), another rhodamine variant further from resonance than $\mathrm{Rh} 6 \mathrm{G}$ with an absorption maximum at $500 \mathrm{~nm}$;

(iv) a Benzotriazole dye (3-methoxy-4-(5'-azobenzotriazolyl)phenylamine, dye $\sharp 2$ of Ref. [88], denoted BTZ here), a smaller dye with absorption close to the $\mathrm{UV}(\approx 400 \mathrm{~nm})$ and therefore even further from resonance at $633 \mathrm{~nm}$; and

(v) Benzotriazole (BTA), a smaller compound with a single aromatic ring, absorbing in the UV and therefore non-resonant.

These compounds are typically good SERS analytes, since they tend to adsorb efficiently on gold and silver surfaces. BTZ and BTA are believed to adsorb covalently to Ag through the triazole group [88]. CV, Rh123 and Rh6G have appeared in a great number of the SERS studies in the literature, and are attracted to the negatively charged citrate colloids as a result of their net positive charge.

It is sometimes assumed that Raman spectra from resonant dyes are difficult to obtain because of the overwhelming fluorescent background. At $633 \mathrm{~nm}$, this was indeed the case in our experiments for other dyes such as methylene blue and nile blue. However, this problem can be avoided in at least two situations: (i) for non-resonant dyes (for example BTZ) or dyes in pre-resonance conditions (for example, Rh6G) at $633 \mathrm{~nm}$, and (ii) for resonant dyes with a very fast non-radiative decay (which drastically reduces the fluorescence quantum yield). For example, $\mathrm{CV}$ has a fast non-radiative channel due to fast rotation-isomerization of the arms of the structure around the central carbon atom [89]. For this reason, measurement of the Raman spectrum is possible for CV even under resonant excitation.

\subsubsection{Measurement of Raman cross-sections}

We used the Raman system described in Section 3.1 with a $\times 100$ Olympus water immersion objective (N.A. 1.0) and a $633 \mathrm{~nm}$ HeNe laser for excitation, with a power of $\sim 4.5 \mathrm{~mW}$ at the sample. 


\subsection{RAMAN MEASUREMENT OF SERS PROBES}

Table 4.3: Common SERS dyes and their UV-Vis extinction spectra. The dyes span a range of resonance conditions and structures. All spectra were acquired in water as a solvent. The positively charged dyes are sourced commercially (Sigma-Aldrich) and are in the form of a chloride salt.

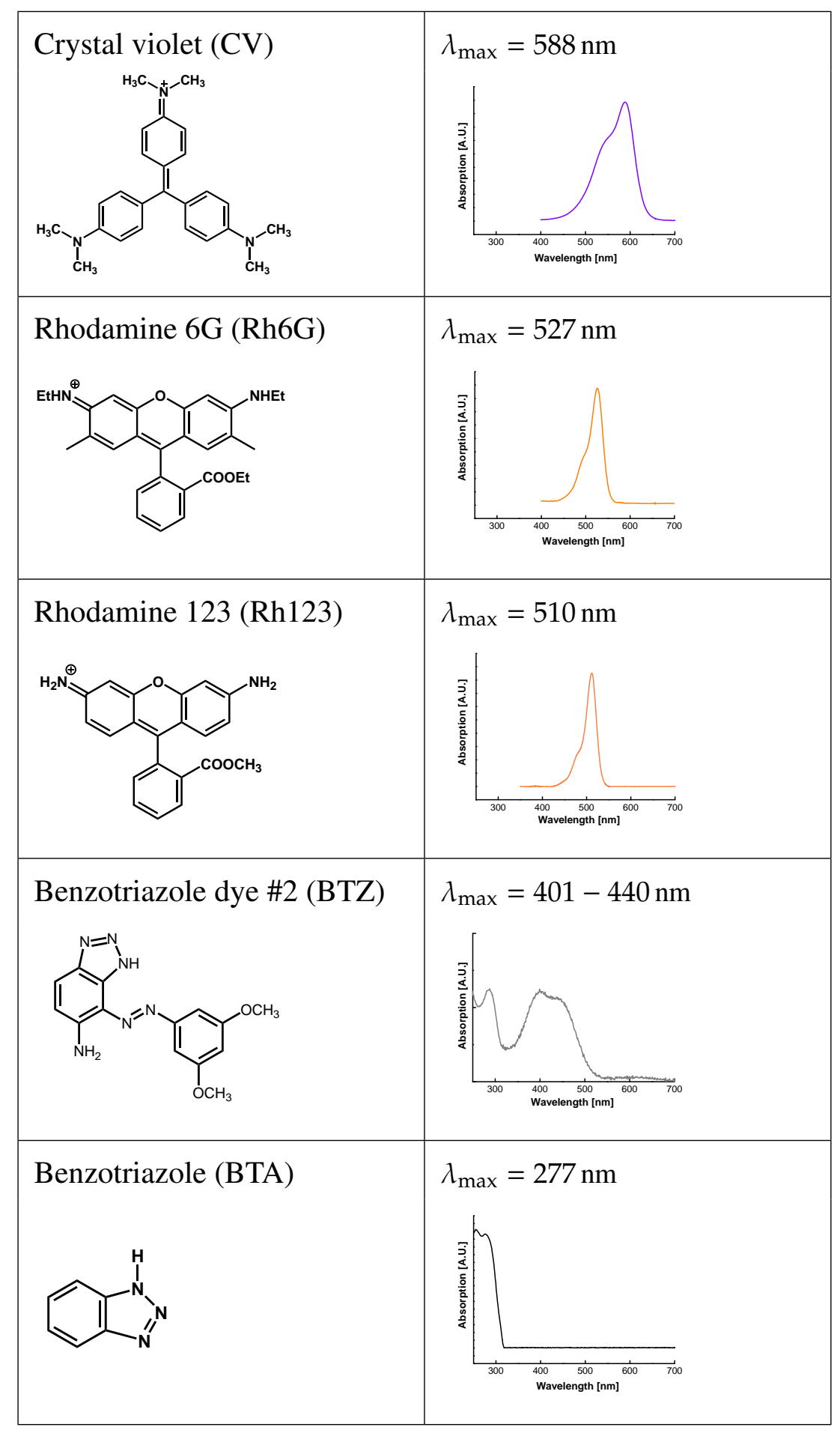


Prior to each measurement, the reference $2 \mathrm{~B} 2 \mathrm{MP}$ was measured under the same experimental conditions. Solutions (in water) of Rh6G, Rh123, CV, BTZ, and BTA, were measured using integration times in the range $150-800$ s. As given in the experimental, all dyes were obtained commercially (Sigma-Aldrich), with the positively charged dyes in the form of a chloride salt. The obtained spectra are shown in Fig. 4.3. Selected peaks from the spectra of the dyes were then fitted using pseudo-Voigt functions (see Appendix A.6.1 for a description), and the integrated intensity was compared to that of the reference $516 \mathrm{~cm}^{-1}$ peak of $2 \mathrm{~B} 2 \mathrm{MP}$ (using Eqn. 4.1 with $c_{\text {Ref }}=8.76 \mathrm{M}$ ).

There are at least two potential problems in such measurements. The first is the possible optical absorption of the dye sample (especially at high concentrations), which would affect the results. To ensure this was not an issue here, we repeated the measurements at two concentrations for CV (10 and $20 \mu \mathrm{M})$ and Rh6G (50 and $100 \mu \mathrm{M}$ ). The second is the possibility of dye photobleaching. In a liquid, dye diffusion is fast and one could expect it to be much faster than photobleaching time, but to make sure this was not an issue, we also repeated the measurements at half the laser intensity. These issues are irrelevant for BTA and BTZ as they are far from resonance, and concentrations of $100 \mathrm{mM}$ and $1 \mathrm{mM}$, respectively, were used. As shown in Fig. 4.3, the Raman spectra are of sufficient quality to extract reliable integrated intensities. A characteristic water background is apparent but nonetheless some peaks can be observed above the noise.

The measured absolute Raman cross-sections for the main Raman peaks of these five compounds are summarized in Table 4.4. 


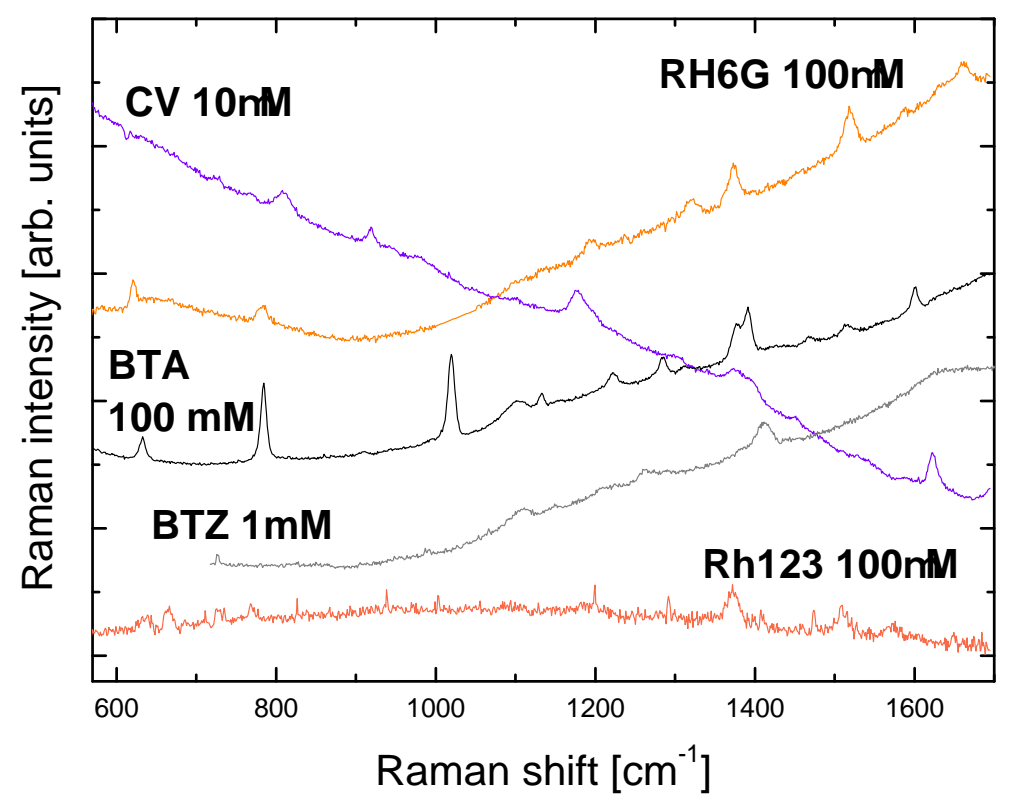

Figure 4.3: Raman (non-SERS) spectra from the analytes characterized here (various concentrations, $c$, and integration times IT): CV, $c=10 \mu \mathrm{M}$, IT= $150 \mathrm{~s}$; Rh6G, $c=$ $100 \mu \mathrm{M}, \mathrm{IT}=400 \mathrm{~s}$; BTA, $c=100 \mathrm{mM}, \mathrm{IT}=240 \mathrm{~s}$; BTZ, $c=1 \mathrm{mM}, \mathrm{IT}=300 \mathrm{~s}$. These are raw spectra, only offset vertically to fit in the same figure. The background for Rh6G, $\mathrm{BTA}$, and BTZ resembles that obtained from a water sample while the background for CV and $\mathrm{Rh} 123$ is due to fluorescence. 
Table 4.4: Main Raman active modes of CV, Rh6G, Rh123, BTZ and BTA, with experimentally determined non-SERS cross-sections, obtained by comparison of integrated intensities to the $516 \mathrm{~cm}^{-1}$ mode of $2 \mathrm{~B} 2 \mathrm{MP}$. + Indicates peaks are part of a doublet.

\begin{tabular}{|c|c|c|}
\hline & $\begin{array}{l}\bar{v}_{i} \\
{\left[\mathrm{~cm}^{-1}\right]}\end{array}$ & $\begin{array}{l}\frac{d \sigma}{d \Omega}(\text { Non-SERS }) \\
{\left[\mathrm{cm}^{2} \mathrm{sr}^{-1}\right]}\end{array}$ \\
\hline \multirow[t]{5}{*}{$\mathrm{CV}$} & 808 & $3.6 \times 10^{-26}$ \\
\hline & 917 & 1.1 \\
\hline & $1177+$ & 6.1 \\
\hline & $1200+$ & 1.5 \\
\hline & 1621 & 3.6 \\
\hline \multirow[t]{7}{*}{ Rh6G } & 612 & $0.67 \times 10^{-27}$ \\
\hline & 774 & 0.76 \\
\hline & 1185 & 0.60 \\
\hline & 1311 & 1.0 \\
\hline & 1364 & 1.8 \\
\hline & 1510 & 2.4 \\
\hline & 1652 & 1.0 \\
\hline \multirow[t]{6}{*}{ Rh123 } & 636 & $4.7 \times 10^{-28}$ \\
\hline & 769 & 2.1 \\
\hline & 1372 & 10.6 \\
\hline & 1509 & 6.8 \\
\hline & 1572 & 3.4 \\
\hline & 1648 & 2.1 \\
\hline \multirow[t]{3}{*}{ BTZ } & 1108 & $1.0 \times 10^{-28}$ \\
\hline & 1412 & 2.5 \\
\hline & 1617 & 0.87 \\
\hline \multirow[t]{5}{*}{ BTA } & 783 & $3.6 \times 10^{-30}$ \\
\hline & 1019 & 4.7 \\
\hline & $1376+$ & 2.8 \\
\hline & $1390+$ & 2.8 \\
\hline & 1599 & 1.3 \\
\hline
\end{tabular}




\subsection{Discussions}

\subsubsection{Comparison with the literature}

These values, to the best of our knowledge, represent the only experimentally obtained bare Raman cross-sections for these probes, despite their relevance in SERS. Other authors have measured the Raman spectra of SERS probes, but have only presented data on the peak frequencies and relative intensities. For example, Watanabe et al. [86] measured the Raman spectra of Rh6G (at 488 and $1064 \mathrm{~nm}$ ) in $\mathrm{EtOH}$ ) but did not attempt to determine the absolute cross-sections. In terms of a basic comparison between our results and their experimental data, it is apparent that for their data at $1064 \mathrm{~nm}$, most of the peaks $(612,1185,1311,1364$ and 1510 $\mathrm{cm}^{-1}$ ) correspond well in terms of relative intensities, with the exception of the $1650 \mathrm{~cm}^{-1}$ peak, which is considerably smaller (data not shown). For the data of Watanabe et al. at resonance $(488 \mathrm{~nm})$, however, the $1650 \mathrm{~cm}^{-1}$ mode increases dramatically relative to the others $[81,86]$. DFT calculations [86] show this mode to be associated with the backbone of the three-ring xanthene chromophore, so it is perhaps unsurprising that it is strongly affected at resonance. Overall, as they measured Rh6G in ethanol and at wavelengths different from ours (488 and 1064 $\mathrm{nm})$, it is not possible to directly compare even the relative peak intensities.

Sarkar et al. [90] performed aqueous Raman measurements of Rh123 at a concentration of $0.3 \mathrm{mM}$ at $609 \mathrm{nM}$. As might be expected at a wavelength close to that used in our experiments, their data are consistent in terms of both frequency and relative intensities of the peaks. Experimental Raman data also exists for CV [91] taken at various excitation frequencies $(458,488$ and $514 \mathrm{~nm})$ in methanol. Again, as is common in the literature, no attempt is made to quantify the Raman cross-sections and the different solvent and excitation frequency prevents us from making a meaningful comparison. Also, when Raman spectra are measured in SERS studies it is often in a solvent other than water (to improve solubility or to avoid the water background). For the purposes of comparison with the SERS situation, it is preferable to obtain the bare Raman cross-section in water. Although 
comparison of Raman cross-sections for different solvents is possible based on their respective refractive indices, this does not take into account the intermolecular interactions with the solvent.

\subsubsection{General remarks}

The first obvious remark is that these cross-sections span a range of four orders of magnitude, with some of the values much larger than typical Raman crosssections. This is in part due to the relatively large size of these molecules, but above all to resonance or pre-resonance Raman effects. The near-resonant condition for $\mathrm{CV}$ is reflected in the large cross-sections, which are in fact not surprising and are typical of Resonance Raman Scattering (RRS) cross-sections. For example, the cross-section of $\mathrm{Rh} 6 \mathrm{G}$ at resonance was recently predicted [81] to be of the order of $10^{-25} \mathrm{~cm}^{2} / \mathrm{sr}$. For Rh6G, whose absorption peaks at $528 \mathrm{~nm}$ and is negligible at $633 \mathrm{~nm}$, a clear pre-resonance effect is still evident, as shown by measured cross-sections of $\sim 10^{-27} \mathrm{~cm}^{2} / \mathrm{sr}$ (Table 4.4).

The large cross-sections of Rh6G highlight the important fact that even nonresonant molecules can have fairly large cross-sections because of pre-resonance effects. This pre-resonance effect appears to be markedly smaller for Rh123, in which the cross-sections are smaller than Rh6G by an order of magnitude on average, despite similarities in the basic molecular structure. The lower cross-sections for Rh123 are the result of the lower absorption maximum for Rh123, which is further away from resonance than Rh6G by about $\sim 30 \mathrm{~cm}^{-1}$, although the Raman cross-section still obtains somewhat of a "boost" from pre-resonance effects. The effects are present even for BTZ $\left(\sim 10^{-28} \mathrm{~cm}^{2} / \mathrm{sr}\right)$, which has an absorption maximum even further away from excitation, and would by most standards be considered non-resonant. The non-resonant nature of the Raman effect cannot therefore be guessed simply from the probe's absorption properties. As Raman scattering is a two-photon process, resonance with the electronic orbital can occur well before their energies coincide. The actual details of Raman resonance are extremely complicated and relate to factors such as the relative geometries of the 
electronic state compared to the vibrational mode of the molecule. While some features of this process may be accessible to time-dependent calculations, this was not the emphasis of this thesis.

Moreover, as a point of comparison, the Raman cross-section of the $1033 \mathrm{~cm}^{-1}$ mode of methanol (used in previous literature as the standard for normalizing SERS EFs [36]) is only of the order of $1.8 \times 10^{-31} \mathrm{~cm}^{2} / \mathrm{sr}$ at $633 \mathrm{~nm}$. This value is between 30 (for BTA) or $3 \times 10^{5}$ (for $\mathrm{CV}$ ) times smaller than the cross-sections measured here. The large non-SERS cross-sections of such molecules mean that they can, in principle, be observed in SM-SERS experiments with SERS EFs much less than usually claimed.

\subsection{Conclusions}

This chapter has outlined the necessary first step in the accurate quantification of SERS EFs-measurement of the non-SERS cross-sections. By finding a suitable reference compound with a known cross-section we have been able to obtain absolute differential Raman cross-sections by comparison of relative peak intensity using a reference standard. In this process, DFT analysis emerged as an extremely useful tool for validation.

As a result we have identified a robust and convenient reference compound in the form of 2-bromo-2-methylpropane (2B2MP). The physical properties of this liquid (non-toxic, appropriate boiling point) allow for rapid and straightforward measurement.

The reference proved effective in estimating the Raman cross-sections of some simple liquids and later the cross-sections for molecules of particular interest to SERS, such as the common dyes $\mathrm{CV}$ and Rh6G. Although it is often assumed that measurement of resonant dyes is not possible because of fluorescence, we show that it is possible in some cases. The resulting estimated cross-sections represent the only experimental values obtained for typical SERS dyes, despite 
their common use in the field.

The Raman measurements showed a number of interesting features. For example, the dyes Rh6G and CV exhibit non-SERS cross-sections at $633 \mathrm{~nm}$ that are much higher than what may be a priori assumed. Even molecules considered nonresonant such as BTZ show higher cross-sections than expected. This highlights the fact that a proper quantification of the non-SERS cross-section must always be carried out if accurate values of the EF are to be obtained. Assumptions based on the absorption spectra of the molecules are not a sufficient guide as a result of potential pre-resonance effects.

The failure to account for this step already accounts for the enormous EFs of $10^{14}$ sometimes quoted in the literature, which are based on a faulty normalization to the cross-section of methanol, which as a non-resonant molecule has a crosssection several orders of magnitude lower.

In summary, all the elements for a reliable characterization of the non-SERS Raman cross-sections of typical SERS probes have been given and 2B2MP appears as a reliable reference compound for future comparisons among data. The next chapter looks at the subsequent measurement of the EFs for these molecules, along with the necessary background material needed to adequately define the EF in the first place. Again, the reference will be of use in quantifying the SERS signal, and the cross-sections measured in the present chapter will be used as the necessary normalizing factors. 


\section{Chapter 5}

\section{QUANTIFYING THE SERS EF}

\subsection{Background}

The previous chapter has outlined the first step in a rigorous methodology to estimate, as accurately as possible, the SERS enhancement factor (EF) for some common SERS probes. This first step, measurement of the bare Raman (non-SERS) cross-section, is necessary to normalize the intensity measured under SERS conditions to obtain the EF. If we fail to measure it then we ignore the fact that the intrinsic Raman cross-sections of different SERS probes can vary by several orders of magnitude at a given excitation wavelength as a result of pre-resonance effects. In the present chapter, we continue our stepwise approach to measure the EF for some common dyes.

In Chapter 4, we covered the major motivations for accurately quantifying the SERS EF, along with the factors leading to the large spread of reported values in the literature. Accordingly, we restate only the main points here, in order to put our approach in context.

Essentially, the wide variability in EFs quoted in the literature, even for similar systems, can be traced to a lack of care in defining the EF and insufficient rigour in its measurement. To deal with these issues, we seek to implement a more structured protocol including the following points: 
(i) Definition of the SERS EF with a specific purpose in mind;

(ii) Measurement with respect to a reference to obtain the effective SERS crosssection; and

(iii) Accurate characterization of the scattering volume for calibration of SM events.

In the following sections, we describe how we implement these points specifically in terms of an experimental methodology.

\subsection{EF Definitions}

\subsubsection{Considerations}

When attempting to measure the EF, it is critical that we first consider its specific definition. As shown in the background theory (Section 2.4), it is possible to express different definitions depending on the specific system, conditions, and purpose in measuring the EF. In particular, we need to take into account:

(i) The importance of distinguishing between EF definitions that are sensitive to the exact experimental conditions and the SERS probe, and those that are more intrinsic to the SERS substrate and therefore truly represent its performance.

(ii) The distinction between average SERS EFs and SM-SERS EFs; both are important characteristics of the substrate, but one or the other may be more relevant depending on the context or applications.

With this in mind, we discuss some suitable EF definitions for our purposes. As covered in detail in Section 2.4, three types of EF can be defined which cover most SERS situations: the single-molecule enhancement factor (SMEF), the SERS substrate enhancement factor (SSEF), and the analytical enhancement factor (AEF). 


\subsubsection{SERS substrates in this work}

The SERS substrates used in our experiments are aggregated Lee \& Meisel Ag colloids [73]. These colloids can be used as an aggregated solution, or dried on a silicon substrate (as described in Section 3.3.3).

The characteristics of these aggregated colloids are covered in Section 3.3.1. The most relevant features of these colloids for our purposes are that they: (i) provide the largest known enhancements in SERS; and (ii) are among the most inhomogeneous systems available in terms of the size and distribution of the particles, their geometrical arrangement, and the resulting EF distribution [50, 92]. Thus, although they provide us with the best chance of measuring SM signals, they are very difficult to characterize with any exactitude in terms of the considerable variation in enhancement and surface area available for SERS. For this reason, we are unable to determine with sufficient accuracy the parameters relevant to the proper expression of the SSEF (Eqn. 2.20), such as the surface density of the individual nano-structures producing the enhancement, $\mu_{M}$, or the surface density of molecules on the metal, $\mu_{S}$. As such, rather than trying to obtain a rough estimate for the SSEF, we focus instead on measuring the AEF and SMEF.

\subsubsection{Specific EF definitions}

We now remind ourselves of the basic definitions for the AEF and SMEF (Eqns. 2.18, 2.21 from Section 2.4), except we now restate these in terms of the Raman and SERS cross-sections.

(i) The single-molecule enhancement factor (SMEF).

$$
\mathrm{SMEF}=\frac{I_{\mathrm{SERS}}^{\mathrm{SM}}}{\left\langle I_{\mathrm{RS}}^{\mathrm{SM}}\right\rangle}=\frac{d \sigma_{\mathrm{SERS}}^{\mathrm{SM}}}{d \Omega} / \frac{d \sigma_{\mathrm{R} S}}{d \Omega},
$$

where $I_{\text {SERS }}^{\mathrm{SM}}$ is the SERS intensity of the single molecule under consideration, while $\left\langle I_{\mathrm{RS}}^{\mathrm{SM}}\right\rangle$ is the average Raman intensity per molecule for the same probe. For the cross-section form of the equation, $d \sigma_{\mathrm{SERS}}^{\mathrm{SM}} / d \Omega$ and $d \sigma_{\mathrm{RS}} / d \Omega$ 
are the SM-SERS and Raman cross-sections with the meaning described in the background material (Section 2.4).

(ii) The analytical enhancement factor (AEF). Let us consider an analyte solution with concentration $c_{\mathrm{RS}}$, which produces a Raman signal $I_{\mathrm{RS}}$ under non-SERS conditions. Then we can define the AEF as:

$$
\mathrm{AEF}=\frac{I_{\mathrm{SERS}} / c_{\mathrm{SERS}}}{I_{\mathrm{RS}} / c_{\mathrm{RS}}}=\left(\frac{d \sigma_{\mathrm{SERS}}}{d \Omega}\right)_{\mathrm{eff}} / \frac{d \sigma_{\mathrm{R} S}}{d \Omega},
$$

where $I_{\text {SERS }}$ is the average intensity of the SERS intensity for the analyte added at a concentration $c_{\mathrm{SERS}}$ and $I_{\mathrm{RS}}$ and $c_{\mathrm{RS}}$ are the corresponding values under Raman conditions. Here, the SERS cross-section is an effective absolute differential SERS cross-section, which refers to the fact that we measure an average SERS intensity spread over the available hot-spots and enhancements in the system. The magnitude will vary depending on the exact colloid preparation but should be reproducible under those conditions. Note that the concentration scaling inherent in the intensity form of the AEF is automatically accounted for in the determination of the Raman and SERS cross-sections relative to the reference compounds (as given in Eqn. 4.1).

As with measurement of the bare Raman cross-sections, a key aspect of our approach to measuring SERS EFs is obtaining the actual cross-sections in Raman and SERS conditions, rather than just comparing intensities. The reasons for this are covered in detail in Section 4.2.1 but we note here also that obtaining a value for the SERS cross-section allows us to compare the SERS effect directly with fluorescence, for example.

\subsubsection{Experimental approach}

In practise, to measure the AEF we simply measure the molecule of interest under SERS conditions (an aggregated colloidal solution) followed by the reference 2B2MP. Because we use the same immersion objective and optical parameters 
(pinhole, alignment) when measuring the SERS signal and the reference, we ensure the scattering volume and laser intensity are the same.

To measure the SMEF, we have two complementary methods available to obtain an estimate for the SERS cross-section. The first is direct measurement, in which SM events are isolated using the BiASERS technique (see Section 2.3.2). The second is the technique known as temperature-dependent vibrational pumping (TDVP; described in detail in Section 2.5).

As discussed in Section 2.5, the TDVP technique automatically yields an estimate of the total SERS cross-section and thus the maximum achievable SMEF on the SERS substrate, which is not necessarily the case for the BiASERS technique. Additionally, the TDVP technique does not require a careful characterization of the scattering volume but only of the excitation profile. It therefore provides results entirely independent of those obtained using BiASERS.

Figure 5.1 summarizes our basic methodology for measuring the AEF and SMEF. We now turn to actual measurement of the EFs for some common dyes for two substrates of interest (aggregated colloids in solution and those dried on silicon).

\subsection{Experimental Measurement of AEFs}

To measure the AEFs, SERS experiments were carried out for dye concentrations of $5 \mathrm{nM}$ using the protocol described in Section 3.3.2. This preparation implies an estimated 60 dyes per colloid [41] or one molecule per $\sim 200 \mathrm{~nm}^{2}$ on the surface. For the other probes, a maximum concentration of $1 \mu \mathrm{M}$ was used (for BTA). In all cases, these concentrations are well below monolayer coverage, which excludes any influence of multilayer effects on the results. As discussed in the experimental (Sect. 3.3.2), the aim is to use a lower concentration to ensure the analyte does not greatly affect the aggregation process and thus change the substrate properties, particularly the stability, and the resulting EFs. Samples prepared at too high a concentration are found to quickly destabilize, ultimately giving poor signal.

The measurements were carried out with the $\times 100$ water immersion objective, 


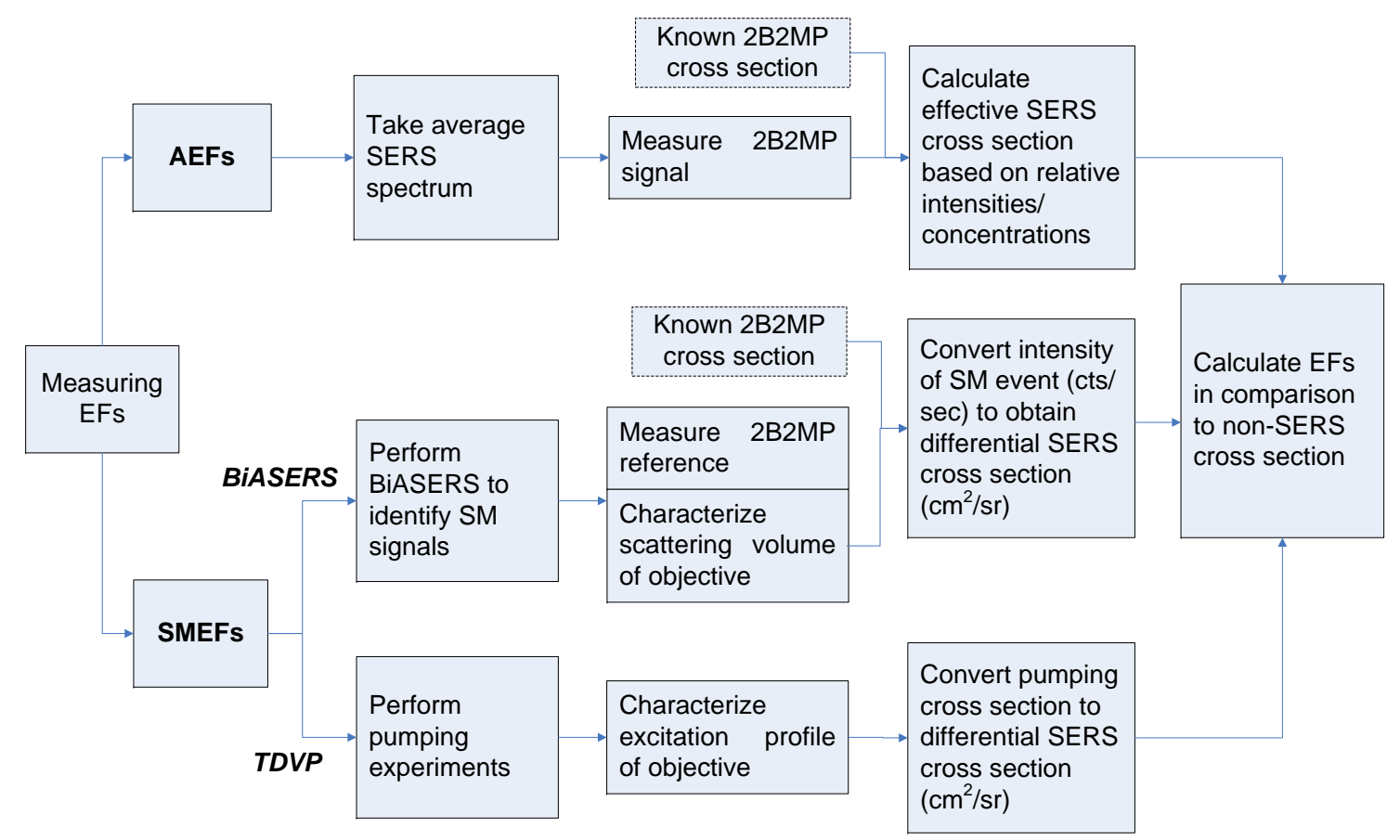

Figure 5.1: Schematic diagram showing the measurement of analytical enhancement factors (AEFs) and single-molecule enhancement factors (SMEFs) obtained by estimating the SERS cross-section with respect to a reference of known cross-section, or by temperature dependent vibrational pumping (TDVP).

which has a relatively small scattering volume $\left(13.0 \mu \mathrm{m}^{3}\right.$; Table 3.1$)$. Long acquisition times are therefore required (minimum 10 minutes here) to average over all cluster types/geometries and therefore eliminate the influence of any fluctuations of the SERS signals. The measured SERS intensity is then compared to the reference compound 2B2MP, and an average (effective) absolute differential cross-section is deduced. This is then compared to the absolute cross-section in the non-SERS case (using Eqn. 5.2). 

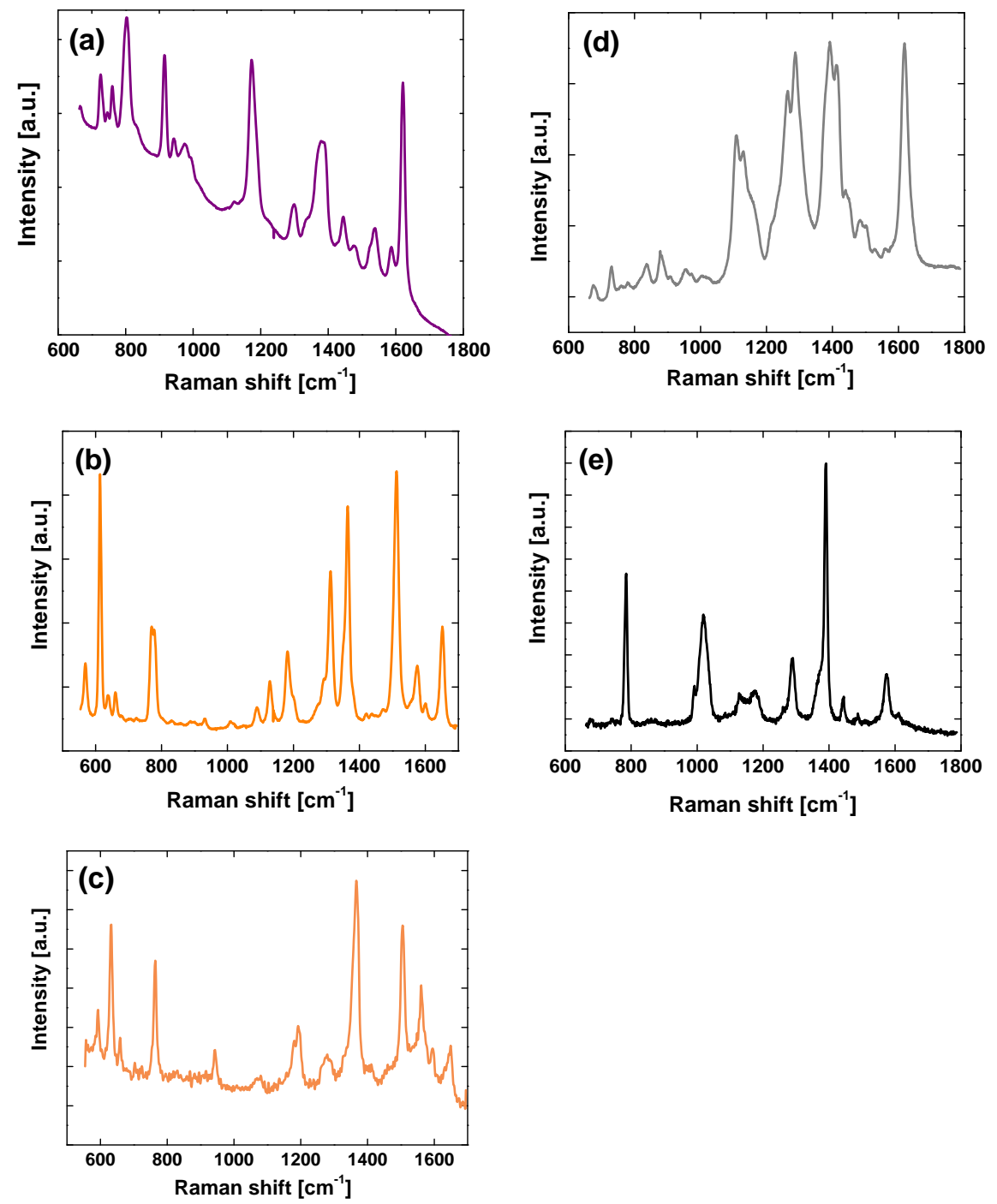

Figure 5.2: SERS spectra for (a) CV, (b) Rh6G, (c) Rh123, (d) BTZ, and (e) BTA. These show average spectra taken over long acquisition times. Some fluorescence is apparent in the background of CV. The excellent spectral resolution and signal to noise displayed in the SERS spectra are in distinct contrast to the bare Raman spectra in Fig. 4.3, demonstrating succinctly the phenomenon of SERS enhancement. 


\subsection{Discussion of the AEFs}

\subsubsection{General observations}

Figure 5.2 shows the average SERS spectra for the five probes. Here, the peaks are narrow and distinct and can be fit with good accuracy. Table 5.1 gives a summary of the results obtained for the five analytes of interest, rhodamine 6G (Rh6G), rhodamine 123 (Rh123), crystal violet (CV), a benzotriazole dye (BTZ, dye 2 of Ref. [88]), and benzotriazole (BTA); see Table 4.3 for structures and absorption profile. Note that the AEFs quoted in this table are specific to the exact preparation conditions of the SERS solution (which was the same for the five analytes). Adding less or more $\mathrm{KCl}$ to the Ag colloids would affect the AEFs; this is normal since we are then not dealing with the same SERS substrate.

If we compare the AEFs among the different molecule we observe a number of important features:

(i) The AEFs are of the same order of magnitude for all five compounds $10^{5}$, suggesting that any form of probe-dependent enhancement (such as chemical enhancement, CE) does not play a significant role for these probes (or is similar in magnitude for all five, an unlikely coincidence).

(ii) These values also demonstrate the importance of a proper normalization to the non-SERS cross-sections for the measurement of SERS EFs. By simply comparing the SERS intensities at the same nominal concentration, one observes that CV exhibits a much greater SERS signal than the others and may conclude its SERS EF is larger. Once normalized to the non-SERS cross-section, the appropriate EFs are obtained. The apparent strength of the CV SERS signals at $633 \mathrm{~nm}$ comes purely from a resonance contribution to the total intensity, which was already present in the non-SERS case. 
Table 5.1: Main Raman active modes of Rh6G, Rh123, CV, BTZ, and BTA, with their experimentally determined non-SERS and SERS frequencies (SERS concentrations are given in the first column). + Indicates peaks that are part of a doublet.

\begin{tabular}{|c|c|c|c|}
\hline & $\begin{array}{l}\bar{v}_{i}(\text { Raman }) \\
{\left[\mathrm{cm}^{-1}\right]}\end{array}$ & $\begin{array}{l}\overline{v_{i}}(\text { SERS }) \\
{\left[\mathrm{cm}^{-1}\right]}\end{array}$ & $\begin{array}{l}\text { AEF } \\
-\end{array}$ \\
\hline Rh6G & 612 & 612 & $5.0 \times 10^{5}$ \\
\hline \multirow[t]{6}{*}{$5 \mathrm{nM}$} & 774 & $768-778+$ & 4.2 \\
\hline & 1185 & $1181(1198)+$ & 4.5 \\
\hline & 1311 & $1312(1292) \dagger$ & 4.4 \\
\hline & 1364 & $1363(1349)+$ & 3.4 \\
\hline & 1510 & 1511 & 3.4 \\
\hline & 1652 & 1651 & 3.1 \\
\hline \multirow[t]{6}{*}{ Rh123 } & 636 & 632 & $3.8 \times 10^{5}$ \\
\hline & 769 & 765 & 6.8 \\
\hline & 1372 & 1366 & 6.0 \\
\hline & 1510 & 1506 & 4.4 \\
\hline & 1572 & 1563 & 4.9 \\
\hline & 1648 & 1649 & 5.5 \\
\hline $\mathrm{CV}$ & 808 & 804 & $1.0 \times 10^{5}$ \\
\hline \multirow[t]{3}{*}{$5 \mathrm{nM}$} & 917 & 914 & 1.8 \\
\hline & $1177(1200)+$ & 1176 & 0.53 \\
\hline & 1621 & 1622 & 0.86 \\
\hline BTZ & 1108 & $1106(1125) \dagger$ & $6.4 \times 10^{5}$ \\
\hline \multirow[t]{2}{*}{$100 \mathrm{nM}$} & 1412 & $1390(1413) \dagger$ & 6.2 \\
\hline & 1617 & 1616 & 8.2 \\
\hline BTA & 783 & 787 & $3.5 \times 10^{5}$ \\
\hline \multirow[t]{3}{*}{$1 \mu \mathrm{M}$} & 1019 & $1035(1021)+$ & 4.5 \\
\hline & $1376-1390+$ & $1369-1394+$ & 5.4 \\
\hline & 1599 & 1579 & 7.8 \\
\hline
\end{tabular}


(iii) The AEFs are relatively low compared to some EFs encountered in the literature, simply because they are average EFs. As will be shown later, the observed SERS signal actually originates from a small sub-ensemble of molecules (those located at hot-spots), experiencing much higher enhancements. If one could position all the molecules at these hot-spots, then much larger average enhancements could be obtained. This is a demonstration of how the issue of spatial averaging affects the definition and magnitude of what we understand by the enhancement factor. As discussed in the background (Sect. 3.3.2), the nature of the error in the AEF is systematic in origin and results from the inherent variability of the substrate synthesis and preparation. In this respect, repeated experiments are found to give variation of up to $\sim 10-20 \%$ even following the same protocol. For an individual measurement, however, comparison is possible among different modes, and the limit in error here is due to the error in measuring the bare Raman cross-section, which is not straightforward for some peaks (such as doublet, multiplets) and is dependent on the choices made when fitting. Again, this shows the dominance of systematic in SERS as opposed to statistical errors.

\subsubsection{Variation of AEFs amongst vibrational modes}

It is interesting to look at the relative peak intensities in SERS conditions. Note that, by definition, the changes in relative peak intensities from Raman to SERS are directly reflected in the variations of the AEFs from peak to peak. If the AEFs of all modes for a given probe are equal, then the relative Raman peak intensities are unchanged in SERS. Let us consider the variation in AEFs for the different modes of each of the probes:

(i) For Rh6G, as seen in Table 5.1, there is a clear trend showing a slight decrease of the AEFs with mode energy. This can be explained within the EM theory of SERS. The EM enhancement factors in SERS have two multiplicative contributions from the underlying plasmon resonance, one at the laser wavelength, and one at the Stokes wavelength [23, 58, 60, 93]. Because of the finite spectral width of the plasmon resonance, it is not possi- 
ble to fulfil simultaneously both resonance conditions to the same degree, especially for large Stokes shifts. This is a well-known effect in SERS, and is the reason why hydrogen stretching modes around $3000 \mathrm{~cm}^{-1}$ tend to be weaker under SERS conditions [22]. It can be shown that the EF is typically a factor of $\sim 1.4-3$ smaller for modes around $1600 \mathrm{~cm}^{-1}$ compared to the low energy modes.

The fact that this small effect is observable for Rh6G must also mean that the vibrational modes (Raman tensors) are not much modified by adsorption on Ag, and that the surface selection rules [94] do not play an important role here. The former assertion is further confirmed by the negligible changes observed in most peak frequencies from normal Raman conditions to SERS. The latter could be explained if the adsorption geometry is random or if the modes have similar symmetries (imposed by the pre-resonance Raman effect).

(ii) Similarly, Rh123 shows a decreasing trend in AEF upon increasing energy, with the exceptions of the 636 and $1648 \mathrm{~cm}^{-1}$ modes. For these modes the observed EFs may be explained by a fixed adsorption geometry, which brings into play the effect of surface selection rules [94]. From DFT results, Sarkar et al. postulated that Rh123 adsorbs via the ring oxygen and the two amine groups, which are found to be regions of appreciable electron density [82]. Conceivably, in that case, the molecule might adsorb directly to the surface via these groups in a particular geometry. Depending on the mode symmetry and its relation to the radiation field, different modes may experience different degrees of enhancement. The Rh123, in contrast to Rh6G, has free amine groups rather than bulky N-ethyl substituents, and would thus be more able to directly bond to the surface in a preferred arrangement and display surface selection effects.

(iii) In the case of BTZ and BTA, the observed EFs can also be explained by a fixed adsorption geometry and surface selection rules. While surface selection rules are always in existence, they will only give rise to changes in particular peak intensities, when the molecule adopts a fixed geometry (or a few more probably geometries) on the surface. Otherwise, any effects will 


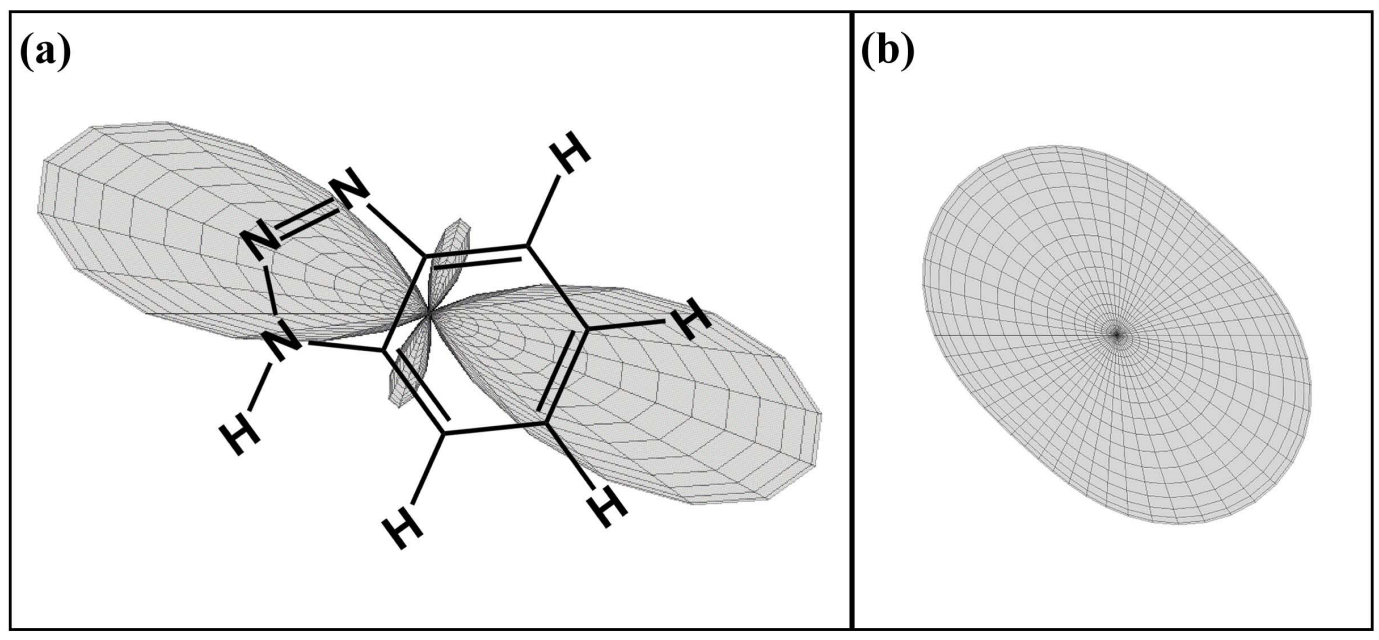

Figure 5.3: Raman tensors for BTA molecule calculated at non-resonance excitation by DFT methods demonstrating potential impact of surface selection rules. The Raman tensor for the (a) 1599 and (b) $1028 \mathrm{~cm}^{-1}$ modes of BTA. The $1599 \mathrm{~cm}^{-1}$ mode is strongly uniaxial along the main molecular axis. If it were to adsorb to the substrate perpendicular to this axis it would be expected that this mode may be enhanced. In contrast, the 1028 $\mathrm{cm}^{-1}$ mode is more isotropic (the same molecular axis applies to the depiction of this mode). These calculations were obtained with the Gaussian package [76] using a CPHF method [96] performed by M. Meyer.

tend to be averaged out. To illustrate this, we focus on the case of BTA. Adsorption is believed to occur through the formation of a covalent bond between $\mathrm{Ag}$ and a $\mathrm{N}$ atom of the triazole group $[83,95]$. Let us assume that the benzene ring extends out of the metal surface and the molecular long axis is therefore perpendicular to the metal and aligned with the local field. Raman modes with a large component along this axis are then favoured compared to other modes. It is possible to show that the Raman tensors of the modes of BTA obtained from DFT calculations are compatible with the observed AEFs. For example, the $1599 \mathrm{~cm}^{-1}$ of BTA is strongly uniaxial along the long molecular axis (whereas the other modes considered here are more isotropic; see Fig. 5.3). This picture of bonding is consistent with the larger AEF observed for this particular mode. 
(iv) Finally, the case of CV is more difficult to interpret. Firstly, the AEFs are markedly smaller than for the other molecules, by a factor of 3-10 depending on the mode. Such a factor is probably too large to be a result of surface selection rules. This could be attributed to a chemical contribution (quenching in this case), but we will show later that under different conditions, the same SERS EFs are measured for CV and Rh6G. We therefore attribute this decrease to either a reduced adsorption efficiency of $\mathrm{CV}$ on $\mathrm{Ag}$ colloids, or to photobleaching; both effects would reduce the AEF. The remaining variations in the relative peak intensities (relative AEFs) can then be attributed to surface selection rules. Additional measurements with a similar compound (malachite green) not shown here demonstrate the drastic effect that photobleaching can have on the estimation of AEFs. Malachite green is resonant at $633 \mathrm{~nm}$ but with a markedly lower photostability than CV [97]. AEFs are reduced even further in this latter case. A meaningful comparison among AEFs for different compounds in a given SERS substrate can only be made if photobleaching plays a minor or negligible role and adsorption efficiencies are comparable.

We conclude from these series of results that: (i) Chemical enhancement does not seem to play a role in the SERS EFs for the five molecules considered here (or at least all the results can be explained from the EM theory of SERS), despite the fact that molecules like BTZ and BTA are believed to adsorb covalently on Ag; and (ii) The EM AEF for the low-energy modes for this colloidal solution at $633 \mathrm{~nm}$ is of the order $\sim 5 \times 10^{5}$. This gives a yardstick order of magnitude for the AEF at low concentrations in Lee \& Meisel colloids which should be easily reproducible by independent studies, provided the same preparation procedure is used.

\subsection{Experimental Measurement of SMEFs}

We now provide several examples of the experimental determination of SMEFs in SERS. We will in particular focus on the dye rhodamine 6G (Rh6G), one of the most widely used probes in SERS. We focus again exclusively on $633 \mathrm{~nm}$ 
excitation, for which the non-SERS properties have been fully characterized.

We reiterate that the experimental difficulties of SM SERS are overcome in this case using two different and recently developed methods, namely: (i) bi-analyte SERS (BiASERS, described in Section 2.3.2), which is used to determine SMSERS conditions; and (ii) temperature-dependent vibrational pumping (TDVP, described in Section 2.5).

\subsubsection{Identifying SM events and obtaining the SMEF}

For direct measurement of the SMEF we use BiASERS to isolate SM events, as described in Section 2.3.2. This technique, combined with relatively low (but not ultra-low) concentrations, offers a compromise whereby a large number of SM events can be obtained together with an easy way of identifying them (by rejecting multi-molecule events).

In a typical BiASERS experiments, one can identify many SM events, with varying intensities. This simply reflects the wide range of SMEFs that are normally encountered for most types of SERS substrates. The observed intensity (and therefore SMEF) depends on many parameters, including whether the molecule is exactly at the hot-spot or slightly displaced, whether the excitation is exactly at resonance or not, or whether the incident polarization is optimally coupled to the hot-spot axis, among other effects. As a consequence, a wide range of SMEFs can be measured in a given sample. In general, the maximum achievable SMEF is the parameter of greatest interest, and this can be measured by sampling the largest SM events. Provided that sufficiently many SM events are acquired, the strongest event must then correspond to the situation in which all parameters (molecule position, plasmon resonance and polarization) are optimal.

To measure the SMEF, we calibrate the SM-SERS event with respect to the scattering volume of our objective as described in Appendix A.5.1. This then necessitates measurement of the reference 2B2MP along with careful characterization of the objective on the day in question using the experiments outlined in Appendices A.4.2-A.4.3. 


\subsubsection{SMEFs in aggregated colloidal solutions}

An aggregated colloidal solution of Ag was prepared as described in Section 3.3.2. To measure the maximum SMEF of Rh6G for this SERS solution, we prepare three solutions: one with a final concentration, $c_{\text {SERS }}$, of $1 \mathrm{nM} \mathrm{Rh6G}$, one with $1 \mathrm{nM}$ nile blue (NB), and a third one where a mixture of NB and Rh6G is added to a final concentration of $1 \mathrm{nM}$ for each dye. NB was selected as a BiASERS "partner" for Rh6G because these two probes can easily be distinguished through their well-defined low energy peaks at $612 \mathrm{~cm}^{-1}$ for Rh6G and $590 \mathrm{~cm}^{-1}$ for NB. We shall, therefore, mostly focus here on the SMEF for the $612 \mathrm{~cm}^{-1}$ mode of Rh6G at $633 \mathrm{~nm}$. Note that the SMEF for NB cannot be calculated here (only its SERS cross-section) since it was not possible to characterize its non-SERS properties because of the overwhelming fluorescence background at $633 \mathrm{~nm}$ excitation. In other words, NB is only being used here as a contrast agent to "distinguish" Rh6G SM events. We use the $\times 100$ water immersion objective for excitation and collection at $633 \mathrm{~nm}$. The incident power was $4.4 \mathrm{~mW}$ at the sample, which corresponds to a maximum excitation density of $7 \times 10^{5} \mathrm{~W} \mathrm{~cm}^{-2}$ at the centre of the beam. Finally, to determine the SMEF, we measure a reference and the scattering volume on the day in question.

Following the same arguments as described in the background to BiASERS (Section 2.3.2), we can isolate SM-SERS spectra from the mixed sample, acquired over a short integration time $(0.05 \mathrm{sec}$ here $)$, in which the signal of one dye or the other is completely absent. We refer to these spectra as SM-SERS events. For the concentrations used here, we estimate that there are approximately $\sim 12$ molecules of each type per colloid. However, the surface area of a hot-spot is typically much smaller than that of the neighbouring metallic surfaces [41, 43], typically $1 \%$ at most. Hence, the chances of a molecule adsorbing at a hot-spot are small, less than 1 in 10. Such hot-spots are formed in colloidal aggregates, which may therefore contain several hot-spots, increasing the chances of observing a SM event. In addition, some aggregates will not be sufficiently resonant to give a SM signal even when a molecule is present at a hot-spot. These considerations are reflected in the observed statistics of SERS signals. Out of 2000 successive spec- 
tra, only about 1 in 20 exhibits a detectable SERS signal. These were analyzed by fitting the $612 \mathrm{~cm}^{-1} \mathrm{Rh} 6 \mathrm{G}$ peak and the $590 \mathrm{~cm}^{-1} \mathrm{NB}$ peak to determine their intensities. By measuring the scattering volume on the day in question and calibrating with respect to the reference $2 \mathrm{~B} 2 \mathrm{MP}$ we can transform this intensity into an effective cross-section (as described in Appendix A.5.1).

The results are plotted in Fig. 5.5. Amongst these events, we can easily identify SM-SERS events for Rh6G and for NB. For these SM events, the apparent crosssection of the event is then equal to the SM SERS cross-section. Using the nonSERS cross-section of the $612 \mathrm{~cm}^{-1}$ mode of Rh6G $\left(6.7 \times 10^{-28} \mathrm{~cm}^{2} / \mathrm{sr}\right.$; Table 4.4), we then deduce the corresponding SMEFs for some of the Rh6G SM-SERS events (see Fig. 5.4). It is interesting to remark that the strongest events in Fig. 5.4 are of a mixed type (not SM-SERS) and this is generally the case in similar experiments.

Such intense mixed events can be attributed to large aggregates, which contain many hot-spots. The BiASERS approach is crucial here for identifying these events and excluding them from the analysis. One minor drawback, however, is that the SMEF for a given hot-spot in these large clusters (which could be expected to be larger than for the smaller cluster showing SM signals) cannot be determined within this approach. We shall come back to this aspect later when discussing temperature-dependent vibrational pumping.

From these results, and several other similar experiments, we conclude that the maximum SMEF for the $612 \mathrm{~cm}^{-1}$ mode of Rh6G achievable for this Ag colloidal solution (for our preparation procedure) is in the range $5-8 \times 10^{9}$, which corresponds to SERS differential cross-sections of $3-5 \times 10^{-18} \mathrm{~cm}^{2} / \mathrm{sr}$. Note that not all the events isolated by BiASERS will always be single molecule in nature, some may include two or more molecules. Thus, an error in this value of a factor of two or so is possible in the downwards direction. However, it is emphasized that in the BiASERS technique we obtain many such potential SM events and on the basis of statistical likelihood we assume that some are certainly single molecule in nature (see Sect. 2.3.2).

These figures are many orders of magnitude smaller than what is usually assumed 


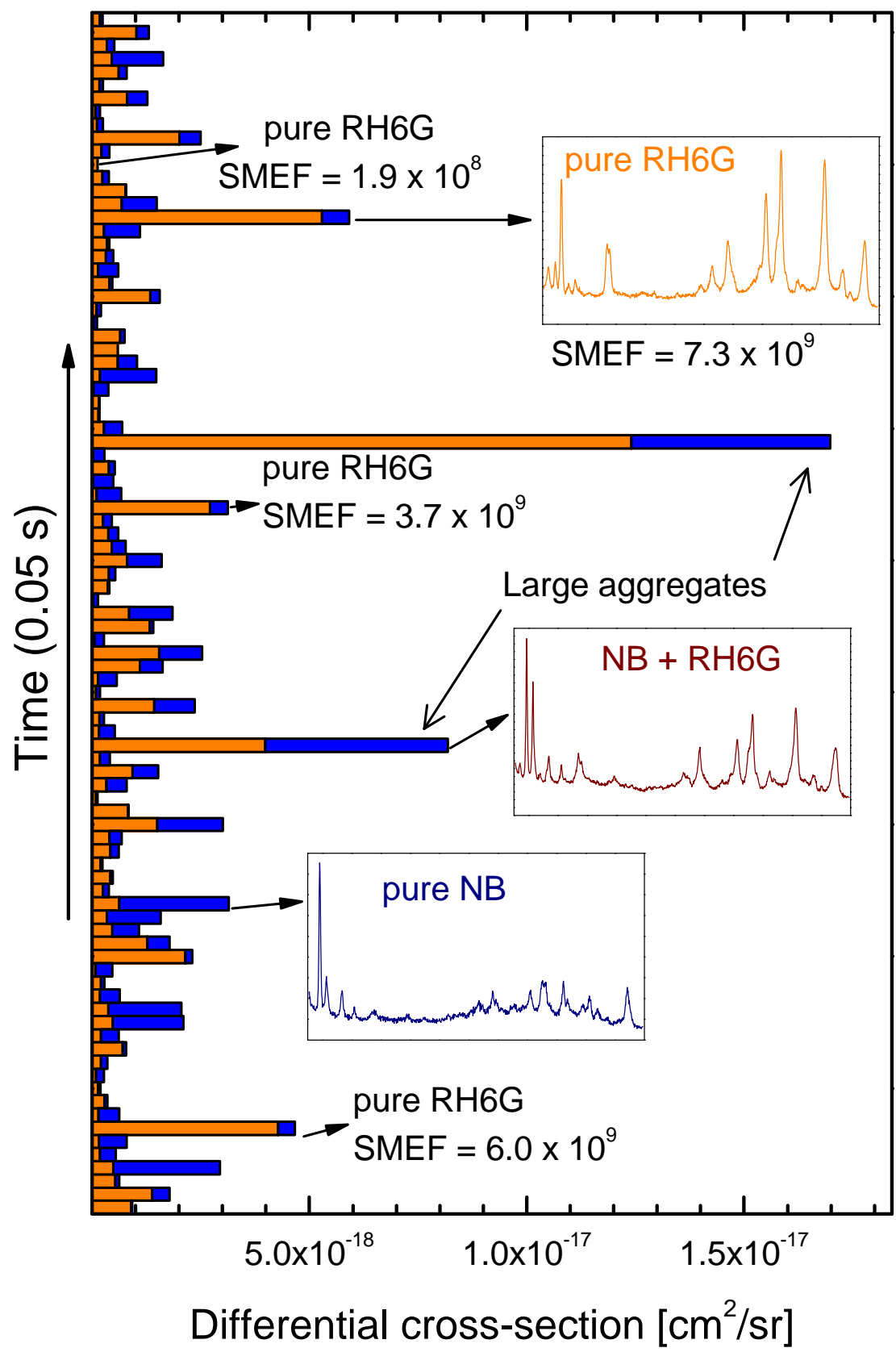

Figure 5.4: BiASERS experiment with Rh6G and NB in a Ag colloidal solution. Each event on the vertical axis corresponds to an individual SERS event (integration time of $0.05 \mathrm{sec}$ ). The effective absolute differential cross-section of the $612 \mathrm{~cm}^{-1}$ mode of Rh6G (orange) and of the $590 \mathrm{~cm}^{-1}$ mode of NB (blue) are shown as (cumulative) bars on the $x$-axis. SERS spectra (from $550 \mathrm{~cm}^{-1}$ to $1700 \mathrm{~cm}^{-1}$ ) are given for pure SM-SERS Rh6G and NB events, and for a mixed event. The SMEF of the $612 \mathrm{~cm}^{-1}$ mode of Rh6G is given for a few SM-SERS Rh6G events. Note that the fitting procedure involves some degree of error and that the existence of a single-molecule event is best confirmed by visual inspection against reference spectra of the two dyes. 
to be necessary. Moreover, the signals giving rise to these SMEFs are still much larger than the detection limit of our system, despite the fairly short integration time of only $0.05 \mathrm{sec}$. In fact, SM detection is possible with a much smaller SMEF, as low as $1.9 \times 10^{8}$ in our case, corresponding to a SERS cross-section of $1.3 \times 10^{-20} \mathrm{~cm}^{2} / \mathrm{sr}$ (see for example the small Rh6G SM-SERS event at the top of Fig. 5.4). This figure could in fact be even smaller with longer integration times. For example, an integration time of $\sim 1 \mathrm{~s}$ would mean a SMEF of only $10^{7}$ and a SERS cross-section of less than $10^{-21} \mathrm{~cm}^{2} / \mathrm{sr}$. Although there are naturally limitations to the integration time for a liquid system because of Brownian motion in the scattering volume, these limitations do not necessarily apply in a dried substrate.

We believe these figures to be the most rigorous values for SMEFs ever reported to date, and they show that claims of EFs of the order of $\sim 10^{14}$ to see single molecules are primarily based on a misconception and a "loose" definition of the $\mathrm{EF}$, together with a neglect of the original non-SERS cross-section with all its contributions from resonance or pre-resonance effects. EFs that are six to seven orders of magnitude smaller than those previously assumed are in fact sufficient to observe single molecules.

\subsubsection{SMEFs in fixed colloidal structures}

As another example of the use of BiASERS to determine SMEFs, we now study the case of colloidal aggregates fixed onto a substrate. A solution was prepared as in the previous section, but with a final $\mathrm{KCl}$ concentration of $15 \mathrm{mM}$ rather than $10 \mathrm{mM}$. The solution in this case is no longer stable, and becomes clear within a few hours. Before this ultimate collapse, we dried a $20 \mu \mathrm{L}$ drop onto a silicon substrate previously coated with a thin layer of poly-L-lysine, prepared as described in Section 3.3.3.

SERS experiments were then carried out in liquid (using a drop of water) with the same $\times 100$ immersion objective as before. Because the substrate is now fixed, we can use a longer integration time ( $1 \mathrm{sec}$ in our case), but the dyes are then more 
likely to exhibit photobleaching. We therefore decrease the power to $0.044 \mathrm{~mW}$ (100-times smaller). The Rh6G SM signals are then stable over minutes, and a small hint of photobleaching is still observed for NB. We acquired a series of SERS spectra by scanning along a line with a $0.2 \mu \mathrm{m}$ step. The signals were analyzed as described in the previous section and the results are presented in Fig. 5.5 .

The results for the dried colloidal aggregates are similar to those obtained for the colloidal solutions with a few significant differences. The SM-SERS crosssection for the $590 \mathrm{~cm}^{-1}$ mode of NB appears to be much larger than the one observed in the previous section. We attribute this to photobleaching effects. Since a small amount of NB photobleaching is evident here for a power density 100fold lower than the previous experiment on liquid colloids, it is likely that at the full power used previously, the NB molecules experiencing the largest EFs would photobleach much faster than the integration time of $0.05 \mathrm{sec}$, thereby reducing the measured SERS cross-section in that case. For the dried colloids, however, the reduction in power means we now are able to sample these larger EFs. This effect highlights the importance of a careful choice of the probe and experimental conditions when estimating and comparing SERS EFs, especially regarding the photo-stability of dyes.

Another relevant point for the fixed colloidal substrates of a map is the lack of statistics. In one line scan, we probe here only 10 aggregates, as seen in Fig. 5.5 , whereas in the same time, we could acquire $\approx 1000$ spectra of individual aggregates in solution, thanks to the intrinsic Brownian motion. This lack of statistics makes it very difficult to determine the reliability of the resultant maximum achievable SMEF. It is also possible to use solid substrates in combination with an analyte solution and an immersion objective. In this case, the equilibrium between analyte molecules at the surface and those in solution allows for the collection of good statistics at a selected hot spot [55]. However, these experiments are dependent on the probe having appropriate adsorption - desorption characteristics and the method was not attempted in the present experiments. 


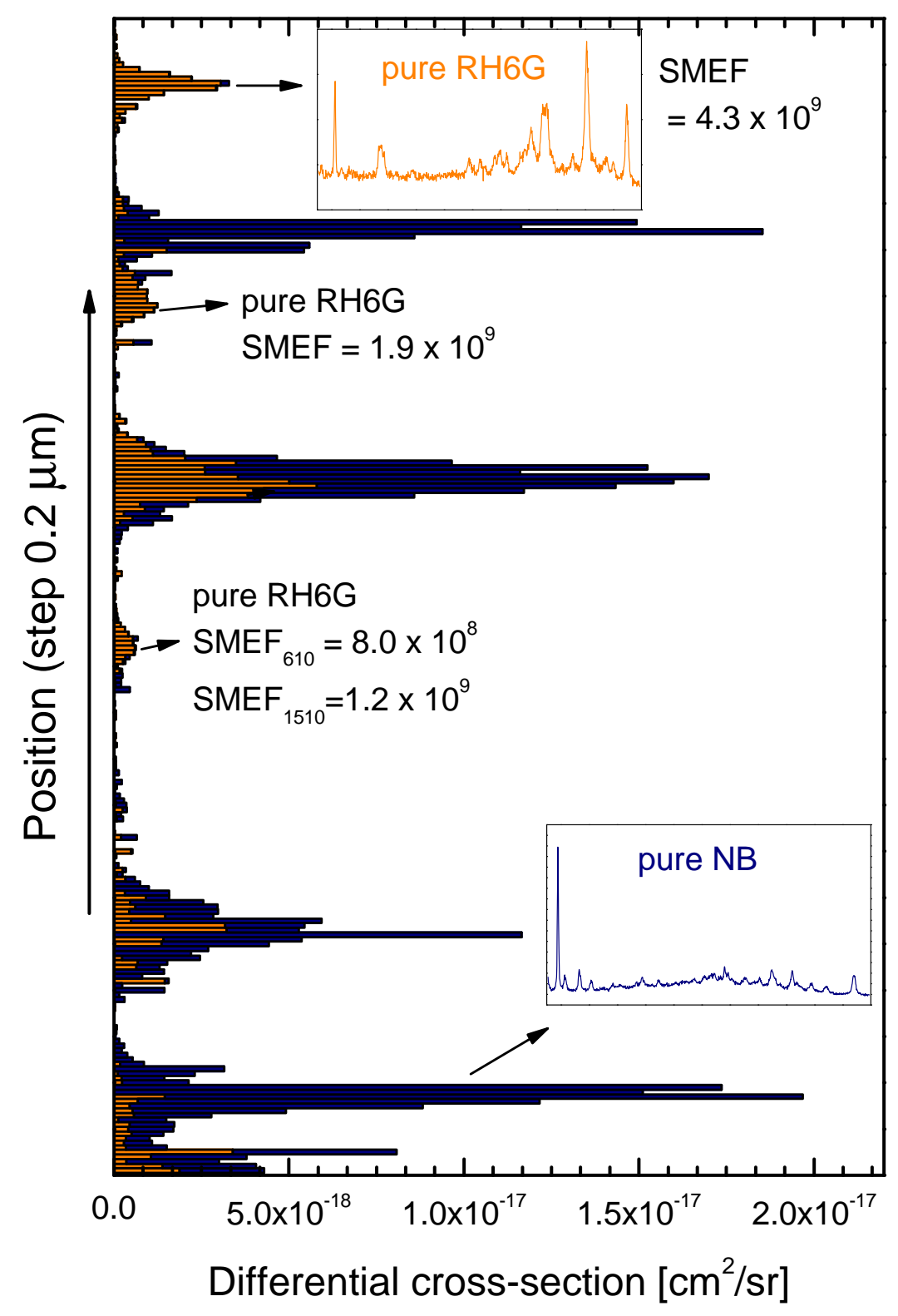

Figure 5.5: BiASERS experiment with Rh6G and NB for fixed Ag colloidal aggregates on a $\mathrm{Si}$ substrate immersed in water. Each event on the vertical axis corresponds to an individual SERS spectrum along the line scan (step of $0.2 \mu \mathrm{m}$ ). The effective absolute differential cross-section of the $612 \mathrm{~cm}^{-1}$ mode of Rh6G (orange) and the $590 \mathrm{~cm}^{-1}$ mode of NB (blue) are shown as (cumulative) bars on the $x$-axis. Examples of SERS spectra (from $550 \mathrm{~cm}^{-1}$ to $1700 \mathrm{~cm}^{-1}$ ) are given for pure SM-SERS Rh6G and NB events. The SMEF of the $612 \mathrm{~cm}^{-1}$ (and $1511 \mathrm{~cm}^{-1}$ in one case) mode of Rh6G is also given for a few SM-SERS Rh6G events. 
It appears that the Rh6G SMEFs obtained in the dried colloid system (Fig. 5.5) are compatible with those of the liquid (Fig. 5.4), but it is expected that the latter values are more statistically sound, notwithstanding that the higher power in this case has led to some photobleaching for NB. (Although NB is not the compound of interest here.) Note that this would not be an issue for more regular/ordered SERS substrates, such as those fabricated by nano-lithography, where more consistent results can be expected.

\subsubsection{Measuring the SMEF using temperature-dependent vi- brational pumping (TDVP)}

\section{Complementarity of TDVP}

Finally, before discussing the results in their totality, we present an additional measurement of SMEFs using an alternative technique, namely temperature-dependent vibrational pumping (TDVP).

The pumping approach is complementary to the BiASERS technique here for a number of reasons:

(i) Firstly, as described in Section 2.5, it automatically yields an estimate of the maximum achievable SMEF on the SERS substrate, and thereby avoids most of the problems associated with the lack of statistics.

(ii) In addition, the largest aggregates always contain many hot-spots and therefore tend to be excluded from the BiASERS analysis. The SMEF at a hotspot in a large cluster cannot therefore be measured with BiASERS. We will here use TDVP to attempt to measure the SMEF in such large Ag colloid clusters.

(iii) Finally, the TDVP does not require comparison to a reference for the crosssection estimation, and therefore does not need a careful characterization of the scattering volume, but only of the excitation profile. The figures obtained are therefore entirely independent of those obtained using BiASERS. 


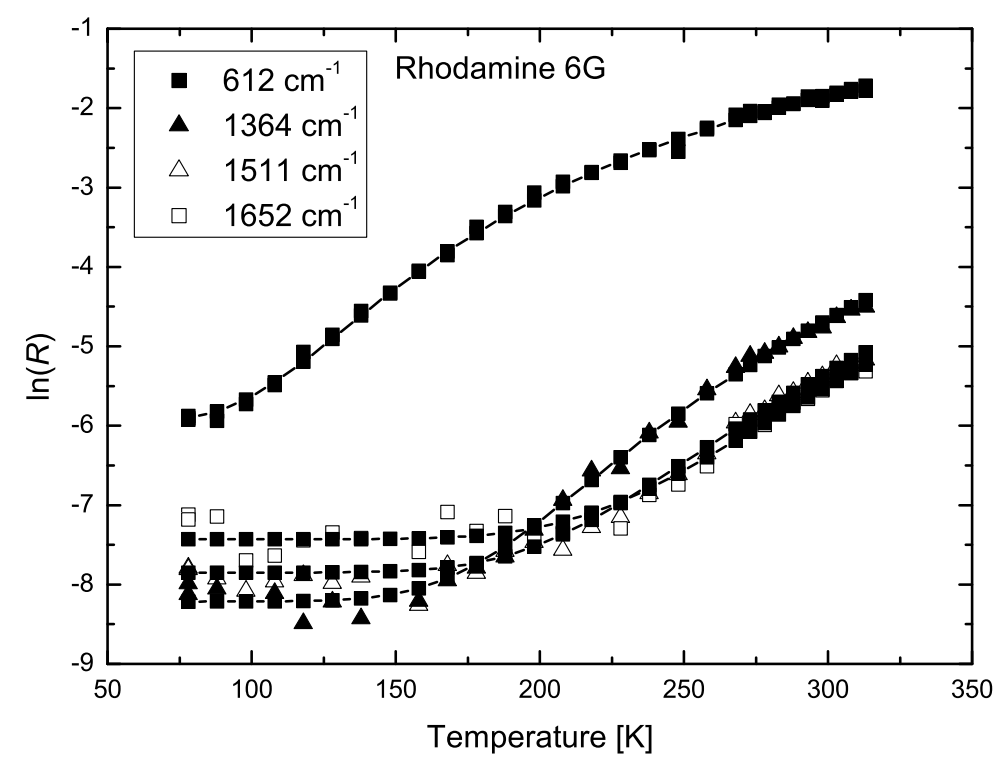

Figure 5.6: The natural logarithm of the anti-Stokes to Stokes ratio $(R)$ is measured as a function of temperature $(T)$ for different Raman modes of Rh6G. The solid lines are fits with the theoretical model of Eqn. 2.24. The curves show a characteristic shape approaching a plateau at low temperatures which differentiates a "thermally dominated" regime at high $T$ 's from a "pumping dominated" regime at low T's. From these pumping curves, estimates of the maximum achievable SERS cross-sections on the substrate can be obtained following the methods described in detail in Section 2.5. See also Refs. [68, 69].

However, this approach is still under development, and as noted in the background (Section 2.5), a number of approximations, not all of them yet rigorously justified, have to be made to extract the SMEF. The most salient of approximations is the estimation of the vibrational lifetime $\tau$ of the mode [69]. The results must therefore be taken with these provisos in mind, but nonetheless the technique adds another dimension to the SMEF problem.

\section{Experimental measurement}

We focus here on two analytes, Rh6G and CV, again at $633 \mathrm{~nm}$. In the experiments, a dried colloidal sample is prepared, and pumping experiments are performed as described in Section 3.4.4, resulting in solid substrates of large Ag- 
colloid cluster aggregates. A $\times 10$ objective was used for excitation and collection. A low magnification objective is advantageous here, since it enables us to probe a larger area (therefore removing any effect of the non-uniformity of the sample), and also ensures the excitation density is low enough to avoid any photobleaching of the analytes.

To measure the maximum excitation density (at the centre of the exciting beam) we conducted "scan-over-an edge" profiling measurements using the $\times 10$ objective (see Section A.4.1 for details and Table 3.1 for objective characteristics). For Rh6G, the excitation density was $I_{0}=9.0 \times 10^{3} \mathrm{~W} \mathrm{~cm}^{-2}$. The larger SERS cross-sections for $\mathrm{CV}$ peaks enabled us to use a lower excitation density of $I_{0}=$ $6.4 \times 10^{2} \mathrm{~W} \mathrm{~cm}^{-2}$. Fig. 5.6 shows the pumping curves obtained for four modes of Rh6G, along with fits to the data following the model described in Section 2.5. The reference peaks used to estimate the cross-sections by the corrected lifetime method were the $1364 \mathrm{~cm}^{-1}$ peak for Rh6G and the $1175 \mathrm{~cm}^{-1}$ peak for $\mathrm{CV}$. The method of analysis to extract the SMEF from TDVP is explained in detail in Section A.5.2. The maximum SMEFs derived from TDVP are summarized in Table 5.2 for representative modes of $\mathrm{Rh} 6 \mathrm{G}$ and $\mathrm{CV}$.

First, we note that the obtained SERS cross-sections of $\sim 10^{-15}-10^{-14} \mathrm{~cm}^{2} / \mathrm{sr}$ are comparable to the cross-sections achieved for single-molecule fluorescence $\left(\sim 10^{-14}-10^{-13} \mathrm{~cm}^{2} / \mathrm{sr}\right.$ [2]. Given that lower cross-sections can be detected in SERS as a result of the narrower signal as compared to fluorescence, these results effectively demonstrate the power of SERS as a detection technique down to the SM level. 
Table 5.2: Summary of the TDVP results for Rh6G and CV. The pumping cross-sections $\sigma_{\text {pump }}$ are derived from a fit to the data as described in Ref. [69]. The maximum differential SM-SERS cross-section $\left(d \sigma_{\text {SERS }}\right) /(d \Omega)$ and the maximum SMEFs on the substrate are then obtained as detailed in Section A.5.2. Note that the maximum SMEF is similar for both molecules. + Indicates peaks that are part of a doublet.

\begin{tabular}{lllll}
\hline \hline & $\begin{array}{l}\overline{v_{i}}(\text { SERS }) \\
{\left[\mathrm{cm}^{-1}\right]}\end{array}$ & $\begin{array}{l}\sigma_{\text {pump }} \\
{\left[\mathrm{cm}^{2}\right]}\end{array}$ & $\begin{array}{l}\frac{d \sigma_{\text {SERS }}}{d \Omega} \\
{\left[\mathrm{cm}^{2} \mathrm{sr}^{-1}\right]}\end{array}$ & $\begin{array}{l}\text { SMEF }_{\text {Max }} \\
\text { Rh6G }\end{array}$ \\
612 & $2.1 \times 10^{-15}$ & $0.50 \times 10^{-15}$ & $7.5 \times 10^{11}$ \\
& $1363 \dagger$ & 5.0 & 1.2 & 6.6 \\
& 1511 & 7.7 & 1.8 & 7.6 \\
& 1651 & 3.7 & 0.88 & 8.8 \\
\hline $\mathrm{CV}$ & 804 & $7.8 \times 10^{-14}$ & $1.9 \times 10^{-14}$ & $5.2 \times 10^{11}$ \\
& 914 & 3.2 & 0.76 & 6.9 \\
& 1176 & 10 & 2.4 & 3.9 \\
& 1622 & 8.8 & 2.1 & 5.8 \\
\hline \hline
\end{tabular}

These figures show that the maximum SMEF on these large Ag-colloid clusters is of the order of $7-8 \times 10^{11}$. It is interesting to note that the same SMEF is obtained for Rh6G and CV (except for small factors $(<2)$ attributed to surface selection rules). This again strongly suggests that there is no chemical contribution to the SERS enhancement in this case (or it is of the same magnitude for both dyes; an unlikely coincidence).

Moreover, the SMEF derived here is around two orders of magnitude larger than that obtained using the BiASERS approach for small clusters. This difference comes from the different nature of the SERS substrate. Within the BiASERS approach, we only probe small or medium-sized clusters. Large clusters are automatically excluded from the analysis since they generally exhibit signals from many molecules. This problem can be overcome using the TDVP approach. The SMEFs derived here of $7-8 \times 10^{11}$ therefore correspond to the maximum achievable SMEFs on Ag (the most SERS-enhancing metal) colloids, in their most aggregated state, i.e. these are the most favourable conditions to produce large SERS 
enhancements. We believe it should therefore be viewed as the upper limit of what is achievable with SERS, and note that this figure is consistent with theoretical maximum EFs (Section 2.2.2). This upper limit is still at least 2 orders of magnitude smaller than the "conventional" EFs of $10^{14}$ assumed many times in the past [98].

\subsection{Discussion of the SMEFs}

It is first interesting to note that the approaches of vibrational pumping and $\mathrm{Bi}$ ASERS are complementary techniques to probing the SERS EF. TDVP [68, 69] is capable of measuring the SERS cross-section independently of the number of molecules by introducing a "non-linearity" to the anti-Stokes signal (the pumping term) and providing a measure which is self-normalizing with respect to the number of molecules.

The SM approach, epitomized by the BiASERS technique, on the other hand, resolves the same issue in a completely different manner: what is known now is the number of molecules producing the signals. If the signal is compared to a reference sample with a known number of molecules and a known cross-section the SMEF follows immediately. The intermediate step of characterizing the normal (non-SERS) cross-section is always needed.

We first look at the case of the maximum SMEFs for the colloidal solution (Fig. 5.4). A number of important features can be identified:

(i) In colloidal solutions, the largest measured SMEF is of the order of $7 \times 10^{9}$. This SMEF is therefore about $10^{4}$ times larger than the AEF measured under the same conditions. This apparent discrepancy has at least two causes. The main origin is the high non-uniformity of the EF distribution [43]. Large EFs occur only at very localized positions (hot-spots) and this is the EF that is measured by the SMEF (largest EF on the substrate). The average EF has been predicted to be 250 - 500 times smaller than the maximum EF [43], so it is expected that the SERS Substrate EF (SSEF) is 250-500 times smaller 
than the SMEF. An additional complication arises from the presence of nonradiative processes [72]. The additional factor of $20-30$ between the SSEF and the AEF can be attributed to the poly-dispersity of colloidal clusters (and therefore of their resonance conditions) and to polarization averaging. The resonance condition and orientation of the cluster in the scattering volume constantly changes. The optimum coupling is automatically selected when the largest SM-events are observed, but averaging over all orientations and resonance conditions results in the observed decrease of the average EF when measuring the AEF.

(ii) Moreover, our results show that SERS from a single molecule can be observed with an integration time of only $0.05 \mathrm{sec}$, and with a differential SERS cross-section of only $\approx 10^{-20} \mathrm{~cm}^{2} / \mathrm{sr}$. This is at least four orders of magnitude smaller than what it usually assumed to be necessary in the literature. The reason for this discrepancy is that these previous estimates were usually based on the requirements of single-molecule fluorescence (SMF). In this case, the signal is much wider, typically $500-1000 \mathrm{~cm}^{-1}$ compared to only $\approx 6 \mathrm{~cm}^{-1}$ for the SERS peak of Rh6G considered here [56]. This narrowing by a factor $\approx 100$ allows detection of signals whose integrated intensity is $\approx 100$ times weaker. Another effect contributing to this discrepancy is the fact that single molecules in solution diffuse much faster than typical colloids, and integration times in SM-fluorescence are therefore much smaller than what is allowed for SERS. This can easily account for another factor of 100 in the detection limit of single molecules. These two important aspects of SM-SERS, narrower peaks and longer diffusion times (or even fixed molecules on fixed substrates), have been largely ignored so far, but they do mean that SM-SERS is possible with cross-sections at least four orders of magnitude lower than for typical SM-fluorescence.

In fact, a cross-section as low as $10^{-21} \mathrm{~cm}^{2} / \mathrm{sr}$ is sufficient to observe SERS from a single molecule with an integration time of $1 \mathrm{sec}$. This corresponds to a SMEF of only $10^{7}$ for Rh6G at $633 \mathrm{~nm}$. For a more resonant molecule, such as $\mathrm{CV}$ at $633 \mathrm{~nm}$, or Rh6G at $514 \mathrm{~nm}$, a SMEF as low as $10^{5}$ may even be sufficient for SM-SERS (if photobleaching is not an issue). This 
is nine orders of magnitude smaller than what was typically assumed in the literature for a long time [98].

(iii) Finally, the results for maximum SMEFs are entirely consistent with the standard electromagnetic theory of SERS enhancement, meaning there is no automatic necessity to refer to a chemical mechanism in the case of the molecules tested.

\subsection{Conclusions}

The last two chapters have detailed the necessary steps for rigorous definition and measurement of the SERS EF, in particular, with respect to two relevant measures, the AEF and SMEF.

We remind ourselves here of the key features that have enabled us to put accurate estimates on the EF, which are necessary to obtain the most accurate values possible:

(i) Careful consideration of the appropriate EF definition;

(ii) Use of a reference standard to calculate cross-sections of Raman and SERS signals;

(iii) Use of BiASERS to unambiguously identify SM events;

(iv) The complementary use of BiASERS and pumping experiments to probe different aspects of the SMEF; and

(v) Careful beam profiling experiments to characterize the excitation profile and scattering volume.

We believe these results settle a matter that has persisted for about a decade since the initial proposal of SM sensitivity in SERS [36, 40], namely, how much enhancement is needed to observe a single molecule in SERS? With the benefit of 
hindsight, we see that the abnormally large enhancements that were proposed in some of the original reports are the natural consequence of several factors, including: (i) the use of an ill-defined EF, where the necessary normalization with respect to non-SERS conditions was sometimes completely ignored; (ii) an underestimation of the bare Raman cross-sections for some common probes, because of resonance or pre-resonance conditions; (iii) the lack of a reliable method to pin down SM events, except for the ultra-low concentration approach which naturally gives rise to some uncertainty [45]; and (iv) a lack of care in the actual measurements themselves in terms of the need to accurately characterize the optical setup.

The analytical enhancement factor (AEF) emerges as a convenient measure of comparison for the average SERS signal obtained for different analytes using a particular substrate definition. The results for the five probes we measured indicated that among these molecules, chemical enhancements were not noticeable. Such a result demonstrates the power of a rigorous quantitative approach in illuminating fundamental aspects of the SERS phenomenon.

The present work, by focusing purely on the EF problem and by addressing each of these points to the best degree possible, has allowed us to obtain accurate EF values that remove some of the confusion surrounding EFs and effectively rule out claims of very high enhancements of $\sim 10^{14}$. One of the problems with such large enhancement factors was that these values are very difficult to justify based on standard electromagnetic theory. This aspect was one of the main arguments in favour of the existence of a chemical contribution to SERS enhancement. EFs reported in this chapter (up to $7-8 \times 10^{11}$ ) are in fact perfectly within our current understanding of electromagnetic SERS enhancements. The comparison of SERS AEFs for different analytes further supports the conclusion that chemical contributions to SERS are less common than generally thought. Finally, these results also show that SM-SERS signals occur more often than previously thought, which was also the conclusion of Ref. [41].

From the perspective of improving the understanding of SERS phenomena, we believe that this work presents the most thorough attempt to date to quantify the EF properly (both theoretically and experimentally), and to pin down and devise a 
proper experimental proof for SM claims. Our view is that these results are a first step towards bringing to a closure a discussion that showed a spread in EFs over several orders of magnitude in the literature for several years.

Another important result of this chapter is the fact that SMEFs of the order of $\sim 10^{7}-10^{8}$ are in most cases sufficient to detect SM signals of probes such as Rh6G and CV. This is 6 to 7 orders of magnitude smaller than what has been claimed for many years in the literature $[36,98]$. We have also shown that, in the best possible conditions, maximum SERS EF are of the order of $10^{12}$. These two results point to the very real potential of detecting SMs of non-resonant molecules. If we can successfully detect SMs for resonant probes with EFs that are several orders of magnitude less than the maximum, then we should, at the maximum available EFs, be able to detect SMs of probes with much smaller Raman crosssections. The extension of this approach to non-resonant probes is presented in Chapter 7.

Meanwhile, we move to a synthetic study aimed at refining the BiASERS experiment to produce ideal partner probes. 


\section{Chapter 6}

\section{ADVANCED BIASERS}

This chapter details a synthetic approach to extend BiASERS to an ideal analyte combination. From a purely experimental point of view, there are many variables that can be optimized in multiple analytes techniques to pin down SM events more efficiently and to simplify the statistical interpretation. The nature of the probes stands out as the first important consideration. In BiASERS it is desirable that the two molecules have different SERS spectra but very similar chemical properties. Critically, the surface chemistry of the probes should ideally be similar in terms of their interaction with the metal substrate which produces the SERS enhancement. It is also important that the molecules have similar SERS cross-sections, and hence similar resonance conditions at the excitation wavelength. Otherwise, the statistics of SM events in SERS could conceivably be biased towards one dye (or the other), depending on the different adsorption or "sticking" properties; this in turn affects the assumed nominal concentrations used in the statistical analysis of the results.

This results in relatively strong constraints for the selection of the probes, and usually a compromise is necessary. In previous BiASERS experiments, a compromise was achieved among the desired properties of specific dyes. In Ref. [41] the "partner probes" were a benzotriazole dye [88] and rhodamine 6G (Rh6G). Reference [54], on the other hand, used n-pentyl-5-salicylimidoperylene and octadecylrhodamine B together with the technique of Langmuir-Blodgett films, while Ref. 
[53] made use of the two related probes 4,4'-bipyridine and 2,2'-bipyridine. More recently $[52,56]$, nile blue (NB) and Rh6G have also been used as successful BiASERS partners for SM-SERS. While all of these combinations proved the bianalyte concept was a successful indicator of SM-SERS, one could argue that the combination of 4,4'-bipyridine and 2,2'-bipyridine in Ref. [53] is perhaps the best among this list, in the sense that it uses two probes which are the closest in both resonance conditions and surface chemistry.

Isotopic editing, with a long and well-established tradition in spectroscopy, appears as one of the best solutions to this problem. The use of different isotopes has a long tradition in science, for example, in carbon dating $\left({ }^{14} \mathrm{C}\right)$, in NMR $\left({ }^{13} \mathrm{C}\right.$, ${ }^{1} \mathrm{H},{ }^{15} \mathrm{~N},{ }^{31} \mathrm{P}$ etc.), or as a clever means to study the metabolism of molecules in living organisms $\left({ }^{14} \mathrm{C}\right)$. In these applications, the central idea is that the isotopic molecule is chemically equivalent to the natural molecule, while having some distinct physical property by virtue of its nucleus (nuclear spin, radioactivity etc). In terms of SERS, if isotopic editing of a useful probe results in a spectrum with distinguishable features compared to the standard (unedited) version of the same molecule, we then have two probes that - for all practical purposes - should have the same chemical properties but can still be distinguished by their SERS signals.

\subsection{Isotopic Editing in SERS}

\subsubsection{Previous studies}

It is interesting to note that isotopic editing is actually not a new concept in SERS either. Zhang and coworkers [99] have already recently demonstrated the utility of isotopic editing for analytical studies in SERS, but did not extend their study to SM SERS or the bi-analyte technique (despite the fact that all the necessary ingredients were already present in their study). In their work, the isotopic probe is simply used as an internal concentration standard.

At the same time, other groups were also considering this approach and, in fact, Dieringer and coworkers [57] applied the concept of isotope editing to BiASERS 
experiments while the present work was in progress. Their work shows a nice example of the potential of this approach to explore the EF distribution of a colloidal sample at varying analyte concentrations. They were also able to observe dynamic fluctuations indicative of competition between molecules at hot-spots. Regardless of the first claim on the use of isotopic probes in BiASERS, such probes can be seen as part of a standard SERS “toolkit", providing us with an effective and reliable standard system for quantification purposes and for a variety of other experiments. In Chapter 7, for example, the use of isotopically edited probes is a critical tool in the optimization of substrates in preparation studies, and subsequently in the actual measurement of SM events with BiASERS.

Our aim was therefore to combine the bi-analyte technique with isotopic editing, thus obtaining the "ideal" BiASERS partners. In terms of the typical dyes used in SERS, it is not possible to simply obtain the isotopically edited molecules commercially. Thus, we looked to obtain the two isotopic analogues of the dye through chemical synthesis. This approach is a good example of the multi-disciplinary nature of SERS, in which we undertook the design, synthesis, and purification of the molecules along with their subsequent use in SERS experiments.

As the target of choice for isotopic editing in SERS, rhodamine stands out as an obvious candidate. The first examples of SM detection were with rhodamine $[36,40]$ and the probe has been a long-standing favourite of the SERS community due to its favourable properties, as outlined in the next section. 


\subsubsection{Rhodamines}

Rhodamines, a class of xanthenes, have been known for a long time, with the first synthesis dating back to the late 19th century [100]. They were initially popular as industrial dyes and have been used recently as laser dyes and fluorescent probes in biological imaging (i.e. fluorescence microscopy, flow cytometry, and fluorescence correlation spectroscopy) as a result of their high quantum yields (e.g. 0.95 for Rh6G) [101, 102].

Table 6.1 shows some of the common commercially available rhodamines. The rhodamine dye is positively charged, with the charge spread across the conjugated dibenzopyrone (xanthene) three-ring structure. The carboxyphenyl group is tilted by about 90 degrees with respect to the xanthene plane to minimize steric repulsion [103]. The positive charge of these dyes is desirable when using them with common colloid preparations such as Lee \& Meisel colloids, because such colloids possess a negative surface charge arising from the surface stabilizing groups (i.e. citrate). This electrostatic interaction between the positive dye and the negative colloids then promotes effective adsorption to the SERS substrate. Optically, the xanthene group has strong absorption in the visible and the carboxyphenyl derivative absorbs in the near-UV [103]. It should be noted that the dyes in Table 6.1 having a free carboxylic acid group $\left(\mathrm{R}_{3}=\mathrm{H}\right)$ are effectively zwitterionic in solution; that is, they have both a positive and negative charge. This tends to hinder their potential use in solution SERS studies, and for this reason it is usually desirable to use an ester version of the dye.

Rh6G, in particular, has been a favourite of the SERS community, due to it having several desirable properties:

(i) A high intrinsic Raman cross-section;

(ii) A formal positive charge only (i.e. not zwitterionic) due to the ethyl ester functionality $\left(\mathrm{R}_{3}=\mathrm{CH}_{2} \mathrm{CH}_{3}\right)$; and

(iii) High photostability. 
Table 6.1: Common rhodamine (Rh) dyes available commercially.<smiles>[R3]OC(=O)c1ccccc1-c1c2cc([R3])c(=[N+]([R])[O-])cc-2oc2cc(N([R7])[R7])c([R3])cc12</smiles>

\begin{tabular}{c|c|c|c|c}
\hline \hline Name & $\mathrm{R}_{1}$ & $\mathrm{R}_{2}$ & $\mathrm{R}_{3}$ & $\mathrm{R}_{4}$ \\
\hline Rh110 & $\mathrm{H}$ & $\mathrm{H}$ & $\mathrm{H}$ & $\mathrm{H}$ \\
Rh123 & $\mathrm{H}$ & $\mathrm{H}$ & $\mathrm{CH}_{3}$ & $\mathrm{H}$ \\
RhB & $\mathrm{CH}_{2} \mathrm{CH}_{3}$ & $\mathrm{CH}_{2} \mathrm{CH}_{3}$ & $\mathrm{H}$ & $\mathrm{H}$ \\
Rh6G & $\mathrm{H}$ & $\mathrm{CH}_{2} \mathrm{CH}_{3}$ & $\mathrm{CH}_{2} \mathrm{CH}_{3}$ & $\mathrm{CH}_{3}$ \\
Tetramethyl Rh & $\mathrm{CH}_{3}$ & $\mathrm{CH}_{3}$ & $\mathrm{H}$ & $\mathrm{H}$ \\
\hline \hline
\end{tabular}

\subsection{Synthetic Strategy for Isotopic Dyes}

\subsubsection{Initial attempts}

In the process of trying to synthesize the dyes, several strategies were attempted. The general goal was to obtain two rhodamine species, chemically equivalent, but having different isotopic composition to hopefully give rise to distinct Raman spectra. The most obvious choice for isotopic variation in a molecule is the replacement of hydrogen with deuterium. After some consideration, we decided on a synthetic attempt to $\mathrm{N}$-alkylate the free amine groups of Rh123 or Rh110 (see Table 6.1) with an alkylating agent (first with a natural hydrogen alkylating agent and then with a deuterated alkylating agent). 


\subsubsection{Ethylation of free amine using ethyl iodide}

The synthetic strategy in this case was to substitute the free amine groups of Rh123 or Rh110 with ethyl groups (or methyl) using an appropriate ethylating (methylating) agent (Scheme 6.1). Complete substitution of the amines by ethyl groups would give a difference in 20 atomic units between the non-deuterated and deuterated species $(\mathrm{d} 5$-ethyl $\times 2 \times 2$ ). Substitution by methyl groups would give a difference of 12 atomic units as an intermediate situation (d3-methyl $\times 2 \times 2$ ). With this in mind, we sought to substitute Rh 110 or Rh 123 with non-deuterated and deuterated alkyl halides (haloalkanes), which can both be obtained commercially at relatively low cost.
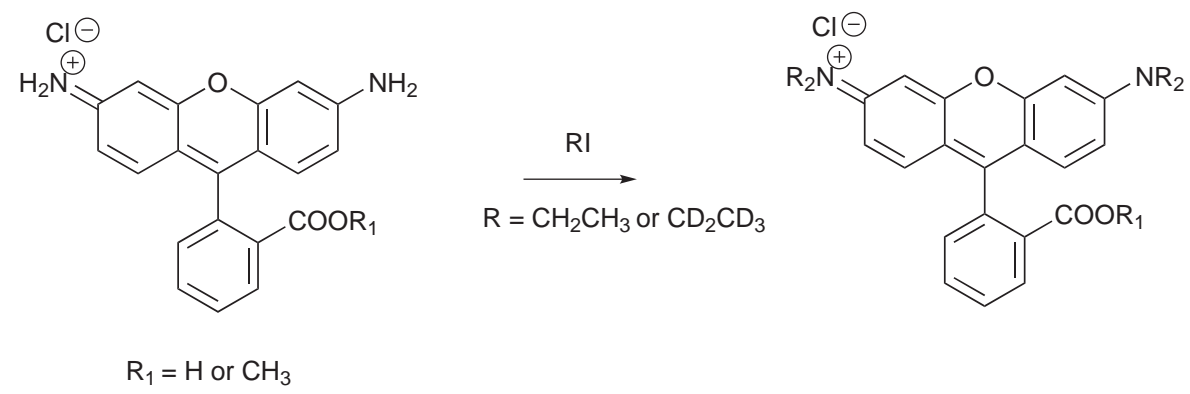

Scheme 6.1: N-ethylation strategy to obtain deuterated and non-deuterated versions of a rhodamine dye. Starting from a rhodamine with free amine groups (Rh123 or Rh110), it should be possible to substitute the free amine group, ideally to completion, using an alkyl halide reagent (in this case ethyl iodide or d5-ethyl iodide).

The above reaction was attempted under a variety of conditions as shown in Table 6.2. As can be seen, many of the reactions were not successful. At higher temperatures, the reaction proceeded to varying degrees but from NMR data it was concluded that complete substitution of the amine had not occurred, and there existed a mixture of mono- and disubstituted amines, which could not be separated by normal- or reverse-phase column chromatography. The use of a range of solvents and the addition of weak bases, such as $\mathrm{NaCO}_{3}$ and $\mathrm{NaHCO}_{3}$, still did not yield the desired product and so after several attempts this strategy was abandoned. A potential factor in the failure of this reaction is the positive charge spread over the rhodamine xanthene structure, which would likely reduce the nu- 
Table 6.2: Attempted N-ethylation of Rh123 to obtain the completely ethylated product (i.e. a RhB-methyl ester equivalent, Table 6.1). As shown, a variety of temperatures and solvents were attempted without success. The mixture of mono and disubstituted products could not be separated by normal- or reverse-phase column chromatography. "eq." represents stoichiometric equivalents.

\begin{tabular}{|c|c|c|c|}
\hline Run & Solvent & Conditions & Result \\
\hline 1 & methanol & 4 eq. EtI, R.T. o/night & no reaction \\
\hline 2 & THF & $\begin{array}{l}4 \text { eq. EtI, } \mathrm{NaCO}_{3(a q .)}, \mathrm{R} . \mathrm{T} \text {. } \\
\text { for } 2 \text { days }\end{array}$ & no reaction \\
\hline 3 & $\mathrm{CH}_{3} \mathrm{CN}$ & $\begin{array}{l}\text { excess EtI, } \mathrm{KOH}_{(a q .)}, \mathrm{mi}- \\
\text { crowave at } 100^{\circ} \mathrm{C} \text { for } 20 \\
\text { mins }\end{array}$ & $\begin{array}{l}\text { mixture of mono- and dis- } \\
\text { ubstituted amines }\end{array}$ \\
\hline 4 & DMF & $\begin{array}{l}\text { excess EtI, } \mathrm{NaHCO}_{3(a q .)} \text {, } \\
\mathrm{AgNO}_{3(a q)}, \text { R.T. o/night }\end{array}$ & no reaction \\
\hline 5 & DMF & $\begin{array}{l}\text { excess EtI, } \mathrm{NaHCO}_{3(a q .)} \text {, } \\
\text { reflux at } 120^{\circ} \mathrm{C} \text { o/night }\end{array}$ & $\begin{array}{l}\text { mixture of mono- and dis- } \\
\text { ubstituted amines }\end{array}$ \\
\hline
\end{tabular}

cleophilicity of the amine nitrogen groups. Alkyl halide substitution of amines involves attack of the amine nitrogen as a nucleophile onto the electrophilic carbon of the alkyl halide [104]. The net positive charge of the rhodamine, which is spread around the entire xanthene structure via the conjugated $\pi$-bonds, may reduce the nucleophilicity of the amine groups and thus hinder nucleophilic attack to the alkyl halide. Although small amounts of pure sample could be obtained through techniques such as quantitative high-performance liquid chromatography, it was considered more suitable to pursue an alternative route. 


\subsubsection{Esterification of free acid using ethyl iodide}

Having had limited success in substitution of the amine, we turned to perhaps the easiest route for obtaining deuterated and non-deuterated analogues of a rhodamine species, esterification of the free carboxylic acid group of RhB (Scheme 6.2). Again, ethyl iodide was used as the reagent. In a microwave reaction at $100^{\circ} \mathrm{C}$, the ethylester of $\mathrm{RhB}$ (named for our purposes $\mathrm{RhE}$ ) was obtained in quantitative yield. The same reaction using deuterated ethyl iodide was also successful, and thus deuterated and non-deuterated partners were obtained. Unfortunately, however, there were insufficient spectral differences in the Raman spectra for the peaks to be resolved (data not shown), suggesting that substitution at the carboxylic acid group does not alter the vibrational modes of the molecule significantly. After not having succeeded with what were initially considered the simplest strategies, we looked to other alternatives.<smiles></smiles>

(1)

RhB

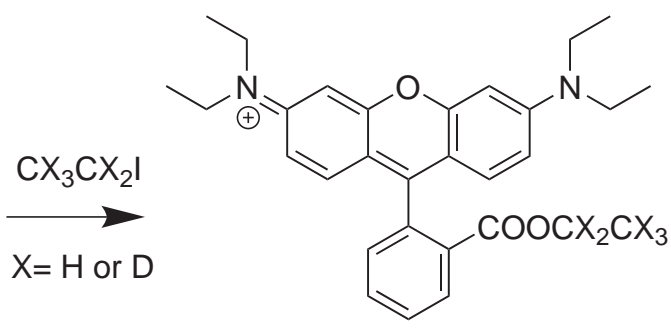

(2)

RhB-ethyl ester (RhE) $[\mathrm{X}=\mathrm{H}$ or $\mathrm{D}]$

Scheme 6.2: Esterification of a free-amine rhodamine precursor (1, rhodamine B) using ethyl iodide (or d5-ethyl iodide) as a reagent to form the ethyl (or d5-ethyl) ester (2).

\subsubsection{Ring-forming condensation reaction (Friedel-Crafts re- action)}

Rather than introducing the deuterium through substitution of an existing rhodamine structure, we decided to proceed via synthesis of the entire rhodamine 
ring structure from component substrates, rather than substitution of the existing framework. The literature reveals several long-existing patents (from 1897 onwards) [100] describing the synthesis of rhodamines through ring-forming reactions between phenol derivatives and anhydrides at high temperatures via FriedelCrafts condensation reactions. In this way, Rh6G is produced by reaction of $o$ ethylamino- $p$-cresol and phthalic anhydride with elimination of water (Scheme $6.3)$.

The initial patent describes heating of the ingredients to $180{ }^{\circ} \mathrm{C}$ using $\mathrm{ZnCl}_{2}$ as a catalyst. However, a revision to the procedure, patented by Abudara et al. [79], used a high boiling-point solvent such as $o$-dichlorobenzene (o-DCB) for the reaction, which would be more desirable in terms of small-scale preparation. The reaction is also convenient for obtaining a deuterated derivative because the precursor anhydride is commercially available as d4-phthalic anhydride. To obtain a probe that is only positively charged, and not zwitterionic, it is necessary to then esterify the free carboxylic acid; as described earlier, zwitterionic probes can be problematic in SERS experiments. The first application of isotopic editing to SERS used a similar approach to the one outlined here [99]. In their work, it was found that the spectra of the resulting deuterated and non-deuterated dyes were sufficiently distinct to be able to resolve (or deconvolute) their individual peaks from a mixture, indicating that these molecules could be used effectively for our purpose. In our particular case, we chose to form the methyl ester in the final step, rather than an ethyl ester, as we had a methylating agent already at hand. Thus, our target molecule was a slight variant of Rh6G, previously unknown in the literature, and named for our purposes Rh6M (M indicates “methyl” ester).

The reaction was successfully performed for both deuterated and non-deuterated forms of the phthalic anhydride precursor on a 0.5-gram scale (see Section 3.5 for details) using a modification of the patent method. Subsequent esterification with dimethyl sulfate $\left(4, \mathrm{R} 1=\mathrm{CH}_{3}\right.$ in Scheme 6.3) provided the methyl ester analogue of rhodamine $6 \mathrm{G}$, formally named 3,6-bis(ethylamino)-9-[2-(methoxycarbonyl)phenyl] xanthylium (hereinafter Rh6M). The identical reaction was then performed for the d4-phthalic anhydride precursor. As shown in Section 3.5, mass spectroscopy and NMR data confirmed the structure and purity of Rh6M and d4-Rh6M, and this 


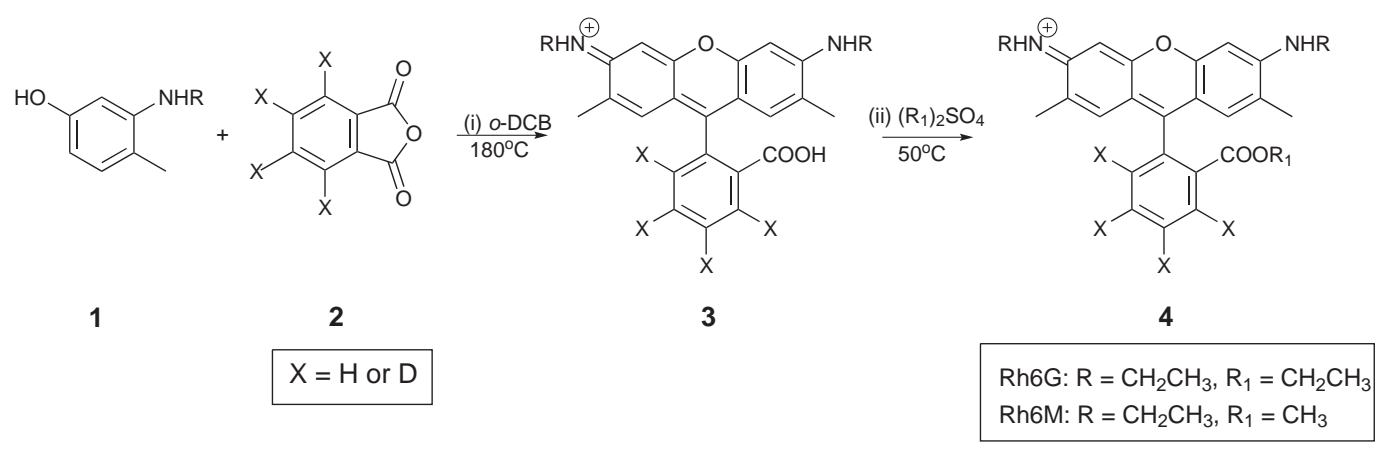

Scheme 6.3: Friedel-Crafts condensation of aminophenol $\mathbf{1}$ and phthalic anhydride $\mathbf{2}$ to give rhodamine isotopic analogues. The phthalic anhydride precursor can be obtained in its natural form $(\mathrm{X}=\mathrm{H})$ and as d4-deuterated $(\mathrm{X}=\mathrm{D})$. (i) Reaction in $o$-dichlorobenzene ( $o$ DCB) at $180^{\circ} \mathrm{C}$ gives the free acid rhodamine 3. (ii) Subsequent esterification of the free acid using an appropriate alkylating agent (in our case dimethyl sulfate) at $50^{\circ} \mathrm{C}$ gives the final product 4 .

data was also largely consistent with previous results for the ethyl ester, $\mathrm{Rh} 6 \mathrm{G}$ [105].

\subsection{Optical Characterization of Isotopic Dyes}

\subsubsection{Fluorescence and extinction measurements}

It was important to first assess whether the Rh6M and d4-Rh6M dyes had the same basic optical characteristics in water, and that both were consistent with the more commonly used ethyl-ester analogue, Rh6G. To this end, extinction and fluorescence measurements were performed on each dye under identical conditions. Figure 6.1 shows the extinction spectra of Rh6M and d4-Rh6M along with Rh6G for comparison. It can be seen that the spectra are essentially identical, with decadic molar extinction coefficients of around $8.4 \times 10^{4} \mathrm{~cm}^{-1} \mathrm{M}^{-1}$ at $\lambda_{\max }=$ $526 \mathrm{~nm}$. Similarly, fluorescence measurements (also shown in Fig. 6.1) taken at $514 \mathrm{~nm}$ excitation show Rh6M, d4-Rh6M and Rh6G exhibit equivalent fluorescence over the measurement range (within 5\%). 


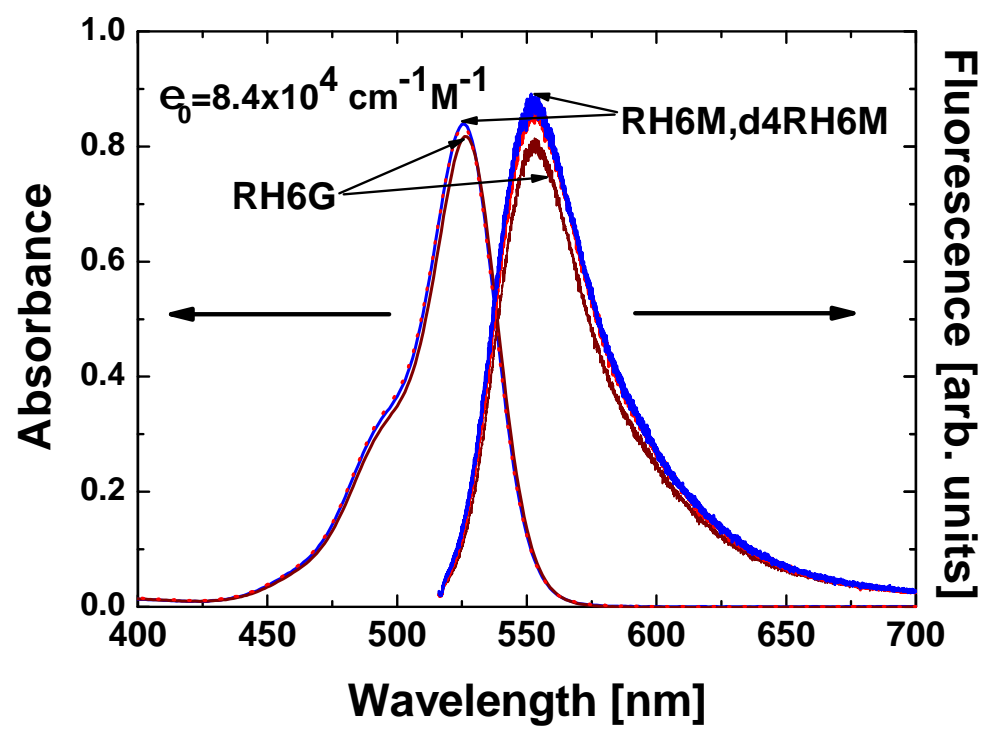

Figure 6.1: UV/Vis extinction and fluorescence spectra of Rh6G, Rh6M, and d4-Rh6M. Rh6G and d4-Rh6M were measured at $100 \mu \mathrm{M}$ in water, while Rh6M was measured at $90 \mu \mathrm{M}$ in water and is scaled for comparison. The d4-Rh6M and Rh6M data are effectively overlapping in the figure indicating identical profiles.

\subsubsection{Bare (non-SERS) Raman spectra and cross-sections}

As covered in detail in previous chapters, it is critical to first examine the molecules under normal Raman conditions before using them in SERS. This allows us to compare the dyes without the confusion of possible adsorption/SERS effects and is a necessary first step for measuring the SERS EFs. As has been stated in detail in Chapter 4, failure to account for the bare Raman cross-sections can lead to errors of several orders of magnitude in the estimation of SERS EFs. In addition to comparing the two isotopic analogues of Rh6M, it was also necessary to ensure that the basic Raman and SERS behaviour of these analogues was largely consistent with that of Rh6G, which is the molecule more familiar to the SERS community.

For the Raman measurements, a $\times 100$ Olympus water immersion objective $(\mathrm{NA}=1.0)$ was used with a $633 \mathrm{~nm}$ HeNe laser for excitation (power $~ 2.3 \mathrm{~mW}$ ). Prior to each measurement, the reference $2 \mathrm{~B} 2 \mathrm{MP}$ was measured under the same experi- 


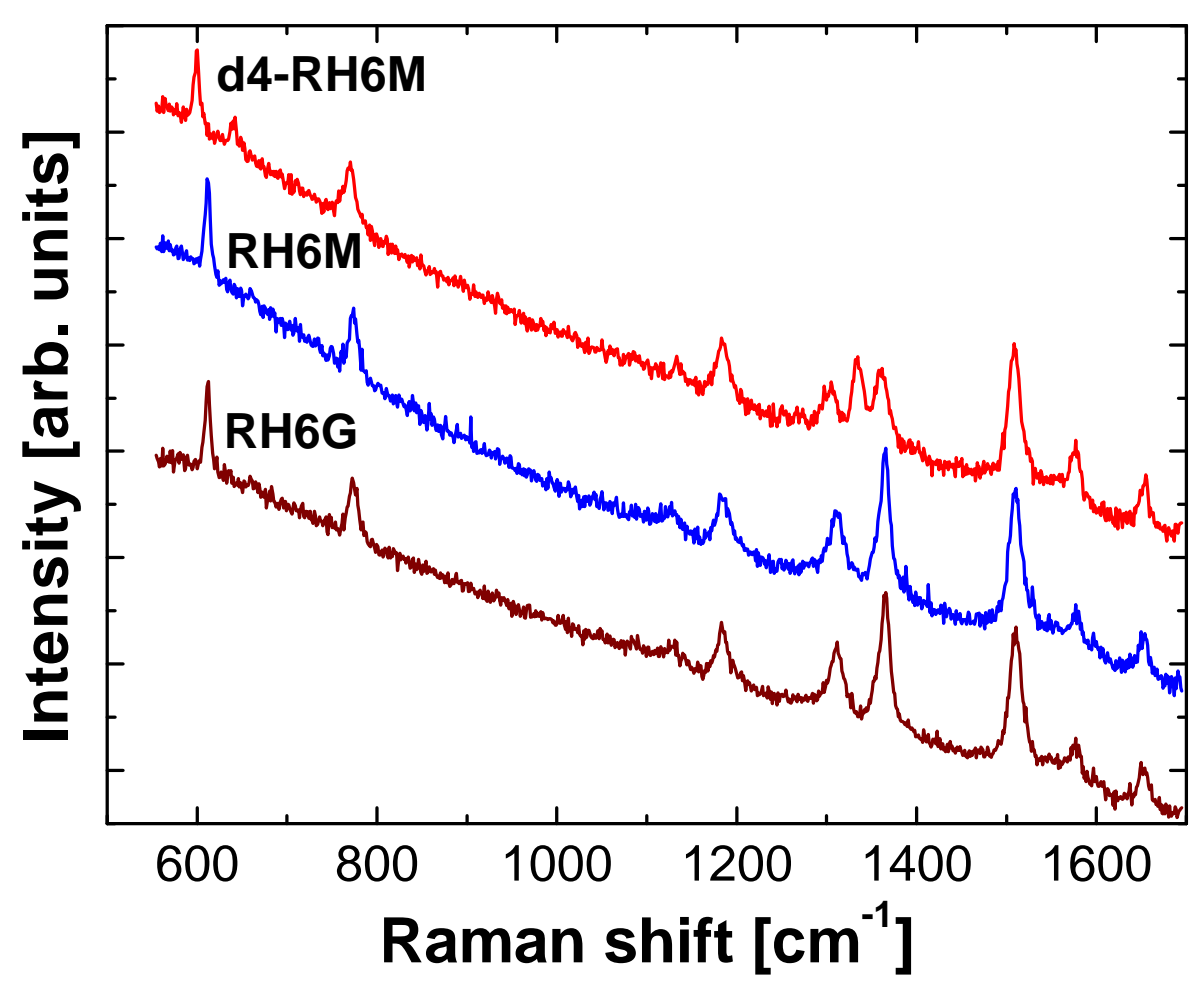

Figure 6.2: Raman spectra (displaced vertically for clarity) of Rh6G, Rh6M, and d4Rh6M. Rh6G and d4-Rh6M were measured at $100 \mu \mathrm{M}$ concentration, while Rh6M was measured at $90 \mu \mathrm{M}$. A water background spectrum taken under the same conditions was subtracted from each spectrum.

mental conditions, as detailed in Chapter 4. Cross-sections are determined with respect to the $516 \mathrm{~cm}^{-1}$ peak of 2B2MP, which has an absolute differential Raman cross-section of $5.4 \times 10^{-30} \mathrm{~cm}^{2} \mathrm{sr}^{-1}$ at $633 \mathrm{~nm}$ [56]. Along with the Rh6M and d4Rh6M dyes, Rh6G was measured for comparison. Figure 6.2 shows the Raman spectra after subtraction of a water background taken under the same conditions. All spectra were acquired with three acquisitions of $600 \mathrm{sec}$. To determine the cross-sections, peak areas were determined by fits to pseudo-Voigt functions (see Appendix A.6.1).

Looking at the spectra of the three dyes, we can see that the Rh6G and Rh6M (non-deuterated) spectra are identical. This agreement is to be expected given that Rh6M is simply a methyl-ester analogue of the ethyl ester Rh6G. In contrast, 
Table 6.3: Comparison of experimental non-SERS differential Raman cross-sections for Rh6G, Rh6M, and d4-Rh6M along with previously measured values from Ref. [56] for Rh6G in parentheses (the discrepancy for the $1652 \mathrm{~cm}^{-1}$ peak of $\mathrm{Rh} 6 \mathrm{G}$ is a result of not subtracting the water background in Ref. [56]; the current results are therefore more reliable).

\begin{tabular}{llllll}
\hline \hline & Rh6G & Rh6M & \multicolumn{2}{l}{$\mathrm{d} 4-\mathrm{Rh} 6 \mathrm{M}$} \\
$\bar{v}_{i}$ & $\frac{d \sigma}{d \Omega}$ & $\frac{d \sigma}{d \Omega}$ & $\bar{v}_{i}$ & $\frac{d \sigma}{d \Omega}$ \\
{$\left[\mathrm{cm}^{-1}\right]$} & {$\left[\mathrm{cm}^{2} \mathrm{sr}^{-1}\right]$} & {$\left[\mathrm{cm}^{2} \mathrm{sr}^{-1}\right]$} & {$\left[\mathrm{cm}^{-1}\right]$} & {$\left[\mathrm{cm}^{2} \mathrm{sr}^{-1}\right]$} \\
\hline 612 & 0.49 & $(0.67) \times 10^{-27}$ & $0.52 \times 10^{-27}$ & 600 & $0.36 \times 10^{-27}$ \\
& & & & 641 & 0.15 \\
774 & 0.65 & $(0.76)$ & 0.60 & 770 & 0.52 \\
1185 & 0.70 & $(0.60)$ & 0.63 & 1185 & 0.63 \\
1311 & 0.99 & $(1.00)$ & 0.94 & 1303 & 0.51 \\
1364 & 1.81 & $(1.80)$ & 1.57 & 1335 & 0.68 \\
& & & & 1361 & 0.90 \\
1510 & 2.00 & $(2.40)$ & 1.87 & 1509 & 1.65 \\
1652 & 0.57 & $(1.20)$ & 0.52 & 1653 & 0.55 \\
\hline \hline
\end{tabular}

the deuterated dye d4-Rh6M is clearly different from its non-deuterated analogue Rh6M, with extra peaks present in the $600-650 \mathrm{~cm}^{-1}$ and $1300-1380 \mathrm{~cm}^{-1}$ regions. These distinct spectral regions are the key to using these dyes in the BiASERS method, for they provide unique fingerprints to identify SM events (in contrast to mixed events).

Focusing now on the bare Raman cross-sections for Rh6M and d4-Rh6M given in Table 6.3, we can see that, apart from the obvious spectral differences in the regions $600-650 \mathrm{~cm}^{-1}$ and $1300-1380 \mathrm{~cm}^{-1}$, the cross-sections of the other modes are consistent. In fact, the spectral differences in the two different regions can be seen as a redistribution of the oscillator strength of the vibrational modes. For example, the $612 \mathrm{~cm}^{-1}$ mode of Rh6M is replaced by two peaks in the case of d4-Rh6M, at 600 and $641 \mathrm{~cm}^{-1}$, and the cross-section of the $612 \mathrm{~cm}^{-1}$ peak of $\mathrm{Rh} 6 \mathrm{M}$ is approximately equal to the combined cross-sections of these two peaks 
in d4-Rh6M. Similarly, in the $1300-1380 \mathrm{~cm}^{-1}$ region, the total area of the two peaks in the Rh6M spectra is similar to that of the three peaks in the d4Rh6M spectra. This redistribution is a result of vibronic levels that may have been degenerate, or close to the same energy (i.e. overlapping peaks), in the natural molecule being sufficiently perturbed by the isotopic substitution as to remove this degeneracy, or split in energy to sufficiently reveal overlapping peaks. As these molecules are complicated and do not have an obvious symmetry it is not straightforward to determine the effect of substitution at a given position. In summary, measurement of the Raman (non-SERS) spectra and cross-sections of the three dyes allows us to conclude that: $(i)$ the Rh6M dye is equivalent (within experimental error) to the more typically used Rh6G dye; and (ii) Rh6M and d4Rh6M have the same Raman cross-sections for most modes (apart from those modes affected by isotopic substitution at $600-650 \mathrm{~cm}^{-1}$ and $1300-1380 \mathrm{~cm}^{-1}$ ). These modified regions can be used to spectroscopically distinguish the two dyes in a BiASERS experiment. The Raman peaks in the other (non-modified) regions are ideal for assessing the similarity of the chemical properties of the two dyes, particularly in terms of surface chemistry (adsorption efficiency and orientation). The next step is to characterize the dyes individually under SERS conditions. 


\subsubsection{Use in individual SERS experiments}

\section{Average SERS properties}

In addition to the dyes having identical chemical properties, it is also essential that they behave the same under SERS conditions, in particular that they adsorb to the SERS substrate to the same degree. Thus, before progressing to BiASERS measurements, the dyes were measured separately under SERS conditions in the many-molecule regime. This enables the characterization of the dyes without the statistical complications of the SM regime. Isotopic editing can result in subtle differences in surface chemistries in general. For example, kinetic isotope effects [106] lead to different "sticking" properties to the surface as a result of the isotopic analogues having different activation energies. In particular, this can occur if the isotopically substituted part of the molecule participates significantly in the surface binding. In general, it is important to check that the dyes have identical properties under SERS conditions.

Both dyes were measured at a concentration of $25 \mathrm{nM}$ using freshly prepared Lee $\&$ Meisel colloids as described in Section 3.3.2. For the purpose of this study, the AEF was used as an estimate for the SERS enhancement of each mode (Section 2.4.3). As in previous chapters, we calculate the AEF by measuring the effective SERS cross-section with respect to a reference (Eqn. 5.2), rather than by direct comparison of spectral intensities of bare Raman and SERS events.

Measurements were carried out using the same setup as described for the bare Raman measurements. A total of 60 spectra were measured for an integration time of $10 \mathrm{sec}$ each in order to wash out any intensity fluctuations associated with the polydispersity of the colloidal SERS substrate. The estimated average AEFs are given in Table 6.4 .

Two features of the AEF data are immediately apparent. The first is that, for each dye, the AEFs are similar throughout all vibrational modes $\left(\sim 1 \times 10^{6}\right)$. The second is that, the AEFs are similar among all three dyes, indicating that the dyes behave the same (chemically) under SERS conditions. The fact that the Rh6M and 
d4-Rh6M dyes exhibit similar AEFs under SERS conditions is a strong indication that: (i) they have the same adsorption efficiency or affinity to the surface; and (ii) any chemical contribution to the SERS enhancement is either non-existent or of the same order of magnitude (note that this is not believed to be the case in this particular system). These AEFs are a little larger than those obtained in Chapter 5 (Table 6.4), indicating the batch-to-batch variability inherent with these colloidal systems. It should be noted that the error in such a value arise largely from systematic causes, chiefly the variation in both the original colloid synthesis and the particulars of preparation of an individual sample. While every effort is taken to ensure identical experimental parameters are used, there may be small variations in the time a sample is left before being measured, or random kinetic variation in the way a sample is initially mixed. On top of this, is the considerable error in measuring the intensities of some of the bare Raman peaks, particularly smaller peaks or those that are part of doublets or multiplets. In this sense, errors of around $10-15 \%$ are expected based on alternative possible fits to the original bare Raman spectra. Thus, as stated in previous chapters, the AEFs should be seen as particular to the specific system and conditions and not necessarily as a characteristic value. The values are reported here to two significant figures, which represents our ability to compare with relative certainty amongst the modes of a given molecule under the same measurement conditions.

In short, Rh6M and d4-Rh6M seem to be chemically identical (in terms of their adsorption properties) and this is an important prerequisite for their use as partners in BiASERS.

\subsection{Single-Molecule (SM) Experiments}

\subsubsection{BiASERS experiments}

The next step is to perform a BiASERS experiment to identify the spectral regions that are sufficiently distinct for determining the statistics of SM events. There are at least two basic criteria that need to be followed: (i) the reference peaks of both 

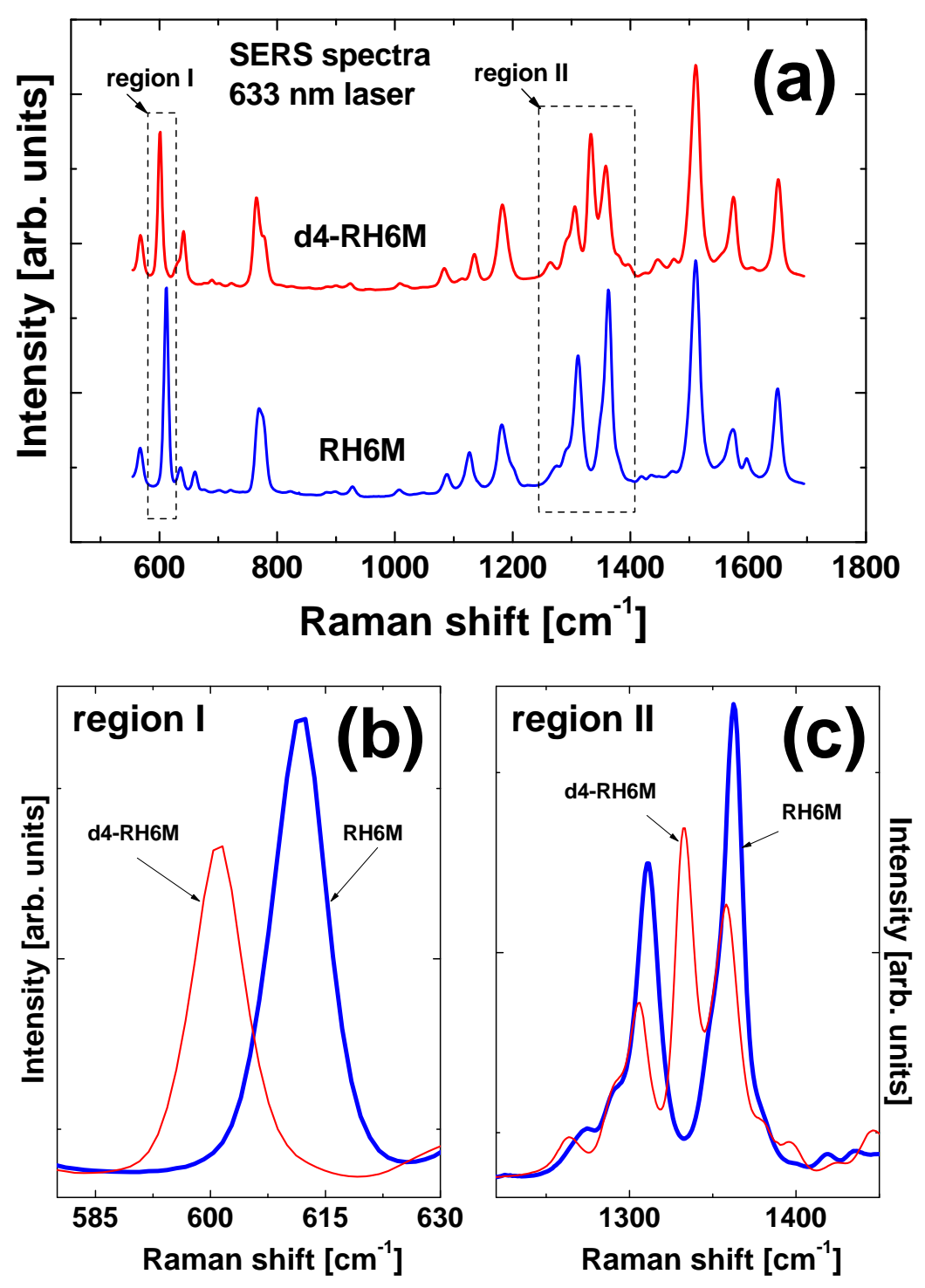

Figure 6.3: SERS spectra of d4-Rh6M (top) and Rh6M (bottom) at $100 \mu \mathrm{M}$ concentration. The spectra show an average of $10^{3}$ spectra with $1 \mathrm{sec}$ integration time. Regions I and II show the distinct spectral differences produced by isotopic substitution; enlarged versions are given in plots (b) and (c), respectively. The isotopic shift of the $\sim 612 \mathrm{~cm}^{-1}$ mode of Rh6M to $\sim 600 \mathrm{~cm}^{-1}$ can be seen in (b), while the doublet in region II in Rh6M splits into a triplet, shown in (c). 


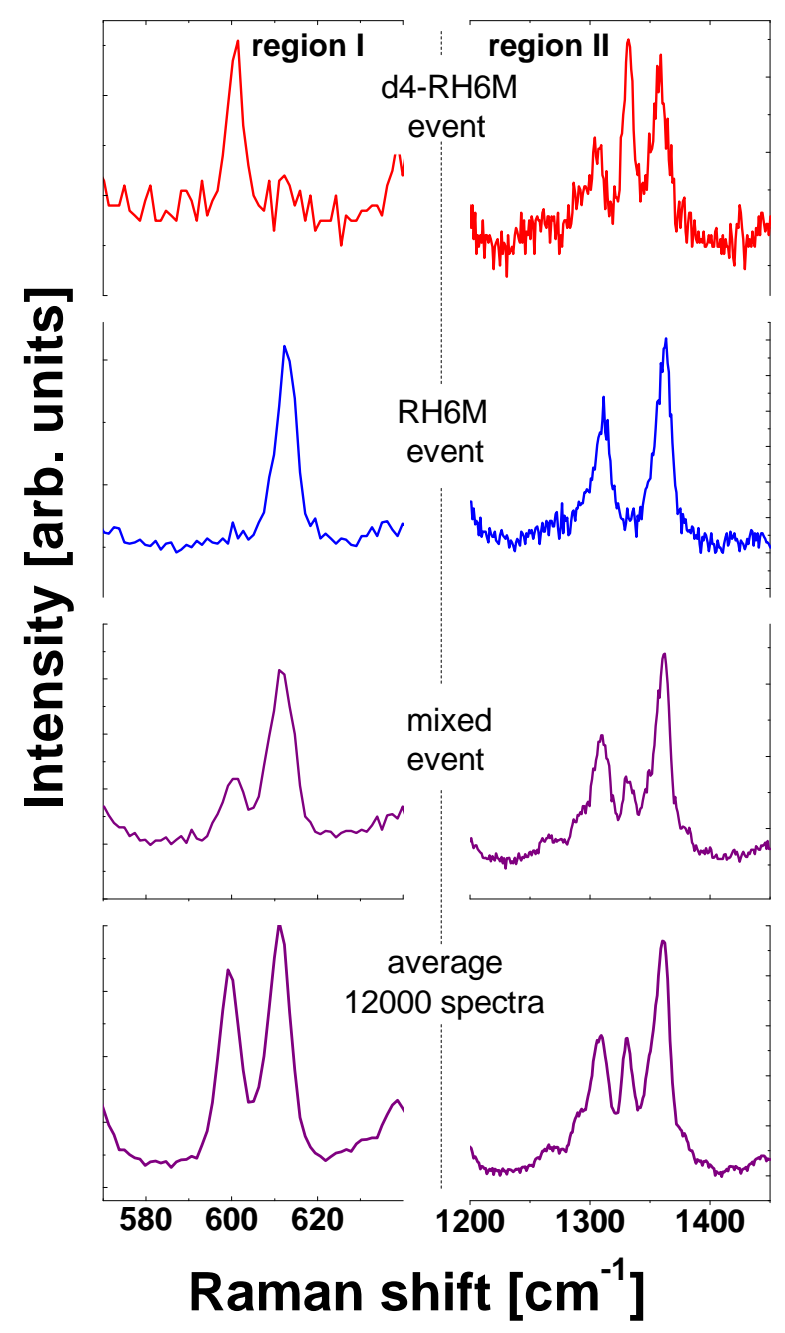

Figure 6.4: SM SERS events for $\mathrm{d} 4-\mathrm{Rh} 6 \mathrm{M}$ and $\mathrm{Rh} 6 \mathrm{M}$ together with a mixed $\mathrm{d} 4$ $\mathrm{Rh} 6 \mathrm{M} / \mathrm{Rh} 6 \mathrm{M}$ event. The average spectrum for $12 \times 10^{3}$ spectra taken at different integrations times is also shown at the bottom. The three topmost spectra (for both regions) show SM-SERS events obtained from the BiASERS data. Note that the "mixed" event is not the same as the average spectrum at the bottom, a situation that is expected if a few molecules (at least one of each type) are not subject to the same single-molecule enhancement factor (SMEF) (one may be closest to the hot-spot, or they could be located at different hot-spots in a small colloidal cluster). 
Table 6.4: Comparison of experimental analytical enhancement factors (AEFs) for Rh6G, Rh6M and d4-Rh6M. Note that these AEFs are specific to the SERS substrate and preparation conditions (which are the same for all three molecules). Note that the relatively larger AEFs measured for the $1651 \mathrm{~cm}^{-1}$ peaks may be an artifact due to the difficulty in fitting this peak in the bare non-SERS Raman spectra. When the peak is a doublet, the frequencies of the second components are given in parenthesis.

\begin{tabular}{|c|c|c|c|c|}
\hline $\begin{array}{l}\overline{v_{i}} \\
{\left[\mathrm{~cm}^{-1}\right]}\end{array}$ & $\begin{array}{l}\text { Rh6G } \\
\text { AEF }\end{array}$ & $\begin{array}{l}\text { Rh6M } \\
\text { AEF }\end{array}$ & $\begin{array}{l}\mathrm{d} 4-\mathrm{Rh} 6 \mathrm{M} \\
\overline{v_{i}} \\
{\left[\mathrm{~cm}^{-1}\right]}\end{array}$ & $\mathrm{AEF}$ \\
\hline \multirow[t]{2}{*}{612} & $1.3 \times 10^{6}$ & $1.2 \times 10^{6}$ & 600 & $1.3 \times 10^{6}$ \\
\hline & & & $640(631)$ & 1.4 \\
\hline $768-775$ & 0.9 & 0.9 & 764 (777) & 1.2 \\
\hline 1181 (1198) & 0.7 & 0.8 & 1181 & 1.1 \\
\hline $1312(1292)$ & 0.7 & 0.7 & $1301(1290)$ & 1.4 \\
\hline \multirow[t]{2}{*}{1363 (1349) } & 0.7 & 0.8 & 1332 & 1.4 \\
\hline & & & 1357 & 1.1 \\
\hline 1511 & 1.0 & 1.0 & 1511 & 1.2 \\
\hline 1651 & 1.5 & 1.6 & 1651 & 1.5 \\
\hline
\end{tabular}

dyes should be different enough to allow the correlations in intensity among different regions to be clearly separated; and (ii) the peaks for the two dyes should ideally not be too far apart in energy ( $\sim 200 \mathrm{~cm}^{-1}$ at most), otherwise the dispersion of the resonance may present a problem during analysis [93]. Figure 6.3 shows the two candidate regions in the SERS spectra of the individual dyes that satisfy these criteria and are therefore suitable for analysis of the BiASERS spectra.

BiASERS experiments with $\mathrm{d} 4-\mathrm{Rh} 6 \mathrm{M} / \mathrm{Rh} 6 \mathrm{M}$ mixtures are performed using the basic BiASERS protocol with a concentration of only $5 \mathrm{nM}$ for each dye (see Section 3.3.2 for details). Again, $633 \mathrm{~nm}$ laser excitation $(3 \mathrm{~mW})$ was used with a $\times 100$ immersion objective and a $200 \mu \mathrm{m}$ confocal pinhole and an integration time of $\tau=0.1 \mathrm{sec}$ The first question is whether we can observe distinct SM events 
for each molecule. Figure 6.4 shows three selected cases (out of $10^{3}$ ) of Raman spectra taken with $0.1 \mathrm{sec}$ integration time, in which characteristic signatures of a single d4-Rh6M event, a single Rh6M event, or mixed events (d4-Rh6M/Rh6M) can be observed simultaneously in both regions, I and II, of the spectra. The data in Fig. 6.4 is equivalent to similar fluctuation data in BiASERS experiments reported before for dissimilar dyes [41,52]. The average shown in the figure represents, as expected, a 50:50 combination of each dye.

\subsubsection{Probing the SM regime in liquids}

With the probes in hand, and proof of their suitability for BiASERS, we now proceed to apply them in a novel BiASERS experiment. The great benefit of BiASERS is that it allows us to identify SM signals in contrast to many-molecule signals. Ideally, we tailor the conditions in such experiments to obtain the maximum number of SM events without too many many-molecule events. This maximizes the statistics for confirmation of SM detection. Determining the conditions that give rise to a majority of SM events can not be guaranteed a priori, but instead must be found by careful experimental optimization. This involves varying the conditions, in particular the concentration, and then repeatedly performing and analyzing the BiASERS experiment. The aim is to be at the transition of the SM regime, in which most of the signals are single molecule in character but the concentration is maximized to obtain the greatest number of these signals.

In a liquid sample, we have an additional parameter to vary - the acquisition time, which controls the time that clusters appear within the scattering volume. Depending on the acquisition time, we can move from sampling several molecules diffusing through the sample volume to sampling single molecules at very short times. In this way we can study the transition from a single molecule, to a few molecules, and finally to many molecules induced by the natural averaging in liquids introduced by Brownian motion. Rh6M and d4-Rh6M serve as the ideal probes for this experiment.

In the BiASERS experiments, $\mathrm{Rh} 6 \mathrm{M}$ and $\mathrm{d} 4-\mathrm{Rh} 6 \mathrm{M}$ were prepared as before and 


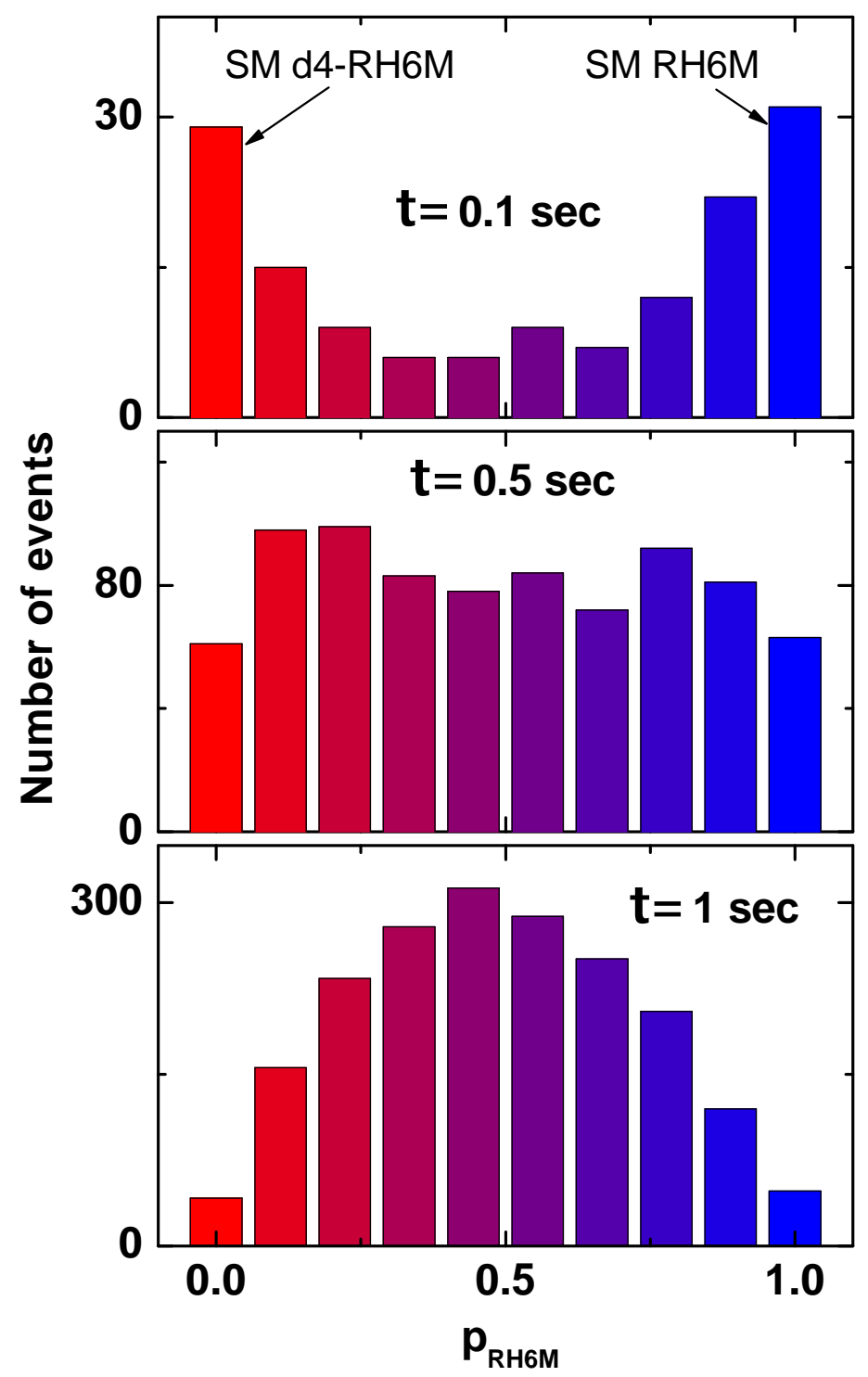

Figure 6.5: Histograms of the relative contribution of one dye ( $p_{\mathrm{Rh} 6 \mathrm{M}}$ in this case) to the total signal obtained by MPCA (see Appendix A.6.3). $P_{\mathrm{Rh} 6 \mathrm{M}}=0(=1)$ indicates a pure d4-Rh6M (Rh6M) event. Weak signal events below a threshold (fixed by the noise level) are removed from the statistics following the procedure of Ref. [52]. The top, middle, and bottom histograms show events for integration times of $\tau=0.1,0.5$, and $1 \mathrm{sec}$, respectively. For $\tau=0.1 \mathrm{sec}$ there are 146 events (out of 3000) above the noise level, while for $\tau=0.5$ and $1 \mathrm{sec}$ there are 810 and 1936, respectively. The statistics for $\tau=0.1 \mathrm{sec}$ are dominated by SM events of either d4-Rh6M or Rh6M, with a few mixed events in between, while those for $\tau=1 \mathrm{sec}$ are mainly dominated by mixed signals. 
measured using the $\times 100$ liquid immersion lens. Sets of data were then obtained for various integration times with several thousand spectra obtained in series for each integration time. These data were then analyzed to show the relative proportions of each dye that contribute to a given spectrum. The data are then boxed in a histogram which shows the frequency at which different proportions of each dye exist (Fig. 6.5. That is, in the histogram, the left hand y-axis shows SM events of one dye, the right hand y-axis shows SM events of the other dye while in between the events are mixed to varying degrees. For data analysis of the $3 \times 10^{3}$ events for each set of events, the Modified Principal Component Analysis (MPCA) method [52] was used for region II. To appreciate the details of the MPCA method, it is recommended that the reader consult Appendix A.6.3. MPCA is a particularly efficient method for analyzing large numbers of data as long as the data is of a sufficient quality. However, it should be noted that other methods, such as fitting directly to the data, would yield the same results.

The results displayed in Fig. 6.5 show the three integration times that best exemplify the transitions between events containing many molecules, to a few, and then a single molecule. The histograms plots the relative contribution of one dye ( $\mathrm{Rh} 6 \mathrm{M}$ in this case) to the total signal, which we call $p_{\mathrm{Rh} 6 \mathrm{M}} \cdot p_{\mathrm{Rh} 6 \mathrm{M}}=0(=1)$ means a pure d4-Rh6M (Rh6M) signal. At integration times of $\tau=0.1 \mathrm{sec}$, only $\sim 146$ events (out of 3000) exhibit a sufficiently high signal-to-noise ratio for a meaningful analysis. These signals are dominated by SM events with either $p_{\mathrm{Rh} 6 \mathrm{M}} \approx 0$ or $p_{\mathrm{Rh} 6 \mathrm{M}} \approx 1$ with a small number of "mixed" events in between. By contrast, at $\tau=1 \mathrm{sec}$, there are 1936 events above the noise level and the spectra are mostly dominated by mixed signals. Arguably, the example in Fig. 6.5 is one of the cleanest experimental examples demonstrating the crossover from a single-molecule to the many-molecule regime in SERS. We observe a gradual transition, which is induced in this particular case by the averaging effect of the integration time combined with Brownian motion in the liquid. As we move to longer times ( $\tau=1 \mathrm{sec}$ ), we obtain a Gaussian profile, which is expected for the random distribution of two species in the many molecule regime. The centring on $p_{\mathrm{Rh} 6 \mathrm{M}}=0.5$ in this case indicates that the probes are at the same concentration and are behaving equivalently in the solution. The transition to the SM regime 
will depend (in general) on the characteristics of the experimental conditions at hand (dry/wet samples, scattering volume, density of hot-spots, etc.) While this is not the main topic here, the change of statistics from one case to another in Fig. 6.5 can also give information on the dynamics of the colloid clusters producing the averaging of the signals. For example, calculations for the $\times 100$ objective and a typical diffusion coefficient for the colloids of $D \approx 7.5 \mu \mathrm{m}^{2} / \mathrm{s}$ give a value for the residence time of a colloid in the scattering volume of $\approx 130 \mathrm{~ms}$ [2]. This estimate, although a rough guide, is certainly consistent with SM behaviour at $\tau=0.1 \mathrm{sec}$, taking the assumption that each colloid is likely to only have one, or a few molecules, at hot-spots large enough to give detectable signals at these low concentrations.

\subsection{Discussion}

\subsubsection{General observations}

As examples of "ideal" BiASERS partners, we have successfully synthesized two isotopic analogues of a rhodamine dye, $\mathrm{Rh} 6 \mathrm{M}$ and $\mathrm{d} 4-\mathrm{Rh} 6 \mathrm{M}$. The two analogues have identical chemical properties but distinct spectra by virtue of their different masses. The dyes were then implemented in a BiASERS experiment to investigate the transition from the single-molecule to the many-molecule regime while varying the integration time in a liquid SERS sample.

The first step in this process was the successful synthesis of the two isotopically edited dyes; the dyes typically used in SERS are not commercially available as isotopic analogues. Once a potential pair of probes has been selected and synthesized, the following key steps are necessary:

(i) Full characterization of the molecules themselves. In particular, it is not always possible to simply assume the Raman cross-sections of isotopically shifted modes to be exactly the same as the unedited counterparts; because oscillator strength redistributions, changes in linewidths, and even splitting 
of modes (or appearance of new modes) can occur.

(ii) It is also possible to test experimentally (rather than assume) that the isotopic substitution effectively does not change the surface chemistry or binding to the surface. This can be done through the comparison of EFs (measured independently under identical experimental conditions) for modes that are not affected by the isotopic substitution in the edited and unedited versions of the molecule.

(iii) Optimization of the conditions such that we are at the transition of the SM regime.

In combination with the BiASERS technique, these probes are a powerful tool in SERS. Their potential application extends from utilization as a standard system in EF quantification of different SERS substrates and preparations, to the detailed investigation of fundamental aspects in SERS, such as the EF distribution of different SERS substrates, or the characteristic clusters in aggregated colloidal solutions.

\subsubsection{Scope of reaction methodology}

It is important to note that the synthetic methodology used in our approach is not limited to a single rhodamine compound. In fact, a variety of rhodamines can be synthesized by the general ring-forming condensation reaction given in Scheme 6.3, by simply using different substituted aminophenol precursors (1: $-\mathrm{NHR}_{1}$, $\mathrm{NR}_{1} \mathrm{R}_{2}$ ); in terms of limitations to the reaction, it is known that the free amine itself $\left(-\mathrm{NH}_{2}\right)$ does not react, and presumably the choice of $\mathrm{R}$ groups would be constrained by steric and electronic effects. Nonetheless, by this method we have access to other commercially available rhodamine species along with the potential to also create new rhodamines.

Similarly, the method can be extended to dyes other than rhodamine. In fact, the dye fluorescein, another common laser dye, is produced by an identical method but using a diphenol precursor rather than an aminophenol precursor. (Scheme 


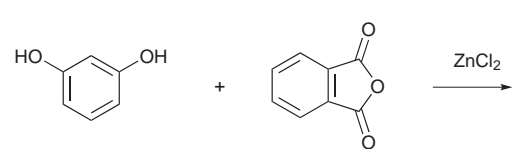

(1)
(2)

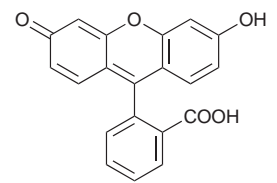

(3)

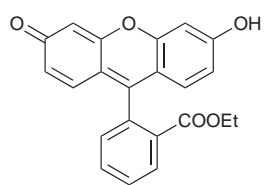

(4)

fluorescein

Scheme 6.4: Synthesis of fluorescein dyes from diphenol (1) and phthalic anhydride (2) precursors.

6.4). Similar Friedel-Crafts reactions can be used in the synthesis of several other triarylmethane and xanthene dyes. Examples include the synthesis of thymolphthalein (a pH indicator) from reaction of thymol and phthalic anhydride [107]. As phthalic anhydride is commercially available as the d4-deuterated analogue this means we have potential access to different classes of dyes as isotopic analogues which may be able to fulfil different applications; fluoresceins, for example, carry a negative charge in solution and may be suitable analytes for investigation of positively charged colloids. The only real limitation here is the dedicated time needed to produce sufficiently pure examples of the dyes in both a deuterated and nondeuterated form. The ability to detect single molecules imposes severe standards on chemical purity in general.

For many of our purposes, and for the SERS community, rhodamine dyes such as the Rh6G analogues synthesized herein (Rh6M and d4-Rh6M) are of the most interest as a result of their reliability and history in SERS experiments.

\subsubsection{Other strategies for obtaining deuterated samples}

As can often happen in scientific research, we discovered in the literature an alternative approach to deuterating common dyes as the present work was coming to a close [108]. In this case, Deb et al. identified a convenient and widely applicable method to obtain deuterated SERS probes including nile blue, the rhodamines B and $6 \mathrm{G}$, malachite green and crystal violet (deuterated by 2, 4, or 6 deuterons depending on the molecule). Their method involved acid catalysis at $90^{\circ} \mathrm{C}$ via an electrophilic aromatic substitution reaction using as a reagent deuterated acid 
chloride in deuterated methanol. By this simple treatment, deuteration could be achieved at particular electron-rich aromatic positions of the main chromophore of the molecule (ortho to the ring oxygen for $\mathrm{NB}, \mathrm{RhB}$ and $\mathrm{Rh} 6 \mathrm{G}$, and ortho to the amine groups for malachite green and crystal violet). Significantly, even molecules changed by only two deuterons gave distinct Raman spectra in comparison to the natural hydrogen version, and crystal violet (with 6 deuterons) gave a shift in one peak of about 40 wavenumbers. This process suggests a straightforward method to obtain isotopically edited dyes with distinct Raman spectra.

\subsection{Conclusions}

This chapter presents the full characterization of an important SERS probe, Rh6M, and its isotopically edited analogue, d4-Rh6M, which can be used hereafter as BiASERS partners for further SM-SERS studies. The relatively large SERS crosssections of the probes together with their identical chemistries and surface interactions with the metal substrate make them ideal probes to explore SM-SERS.

The results of this chapter highlight another important aspect of SM-SERS: It is not enough to have two different SERS probes with identical chemical properties to carry out BiASERS experiments. In addition to the probes, there are parameters that need to be tailored for the observation of SM statistics and these include: (i) the scattering volume of the collecting optics (which will define the dye concentration level that can be used); (ii) the integration time (in connection with the intrinsic diffusion times in the sample); and (iii) the nature of the sample (e.g. whether freely diffusing in a liquid or static). We have demonstrated one of the simplest examples of how the statistics can change from a SM regime (but with sparse events, with only 146 out of 3000 events) to a many-molecule regime, where more statistics are available (1936 out of 3000), in a transition brought about simply by changing the sampling time (as a result of Brownian motion).

It is clear that the use of isotopic editing in BiASERS offers a wealth of information in the investigation of SM-SERS statistics. The thorough characterization of the non-SERS and SERS properties of two such probes (Rh6M and d4-Rh6M), 
together with their application in a simple BiASERS experiment, attempts to contribute to that endeavor.

The work of the previous chapters has provided us with a comprehensive toolkit for exploring SERS phenomena, and the SM regime in particular. In the next chapter, we look to extend this work to the SM detection of non-resonant probes. 


\section{Chapter 7}

\section{NON-RESONANT PROBES}

\subsection{Introduction}

The preceding chapters have led us through a stepwise process for identifying and quantifying the enhancement given in SERS and optimizing the BiASERS experiment for SM detection. The results of Chapter 5 are, we believe, the best estimates of SERS EFs yet to date. Importantly, they also pointed a way towards the SM detection of non-resonant probes. One of the major results of Chapter 5 was that SMEFs of $10^{8}-10^{9}$ were sufficient to see SM signals of the typical dyes used in SERS. In addition, it was found that maximum EFs of up to $10^{11}$ could also be obtained for the same substrate. The natural corollary of these results is that by sampling the hot-spots with the greatest enhancements and by increasing the integration time through the use of a solid substrate, we should be able to measure SM signals for probes with intrinsic cross-sections several orders of magnitude lower than the probes typically examined in SERS; that is, it should be possible to detect single molecules of non-resonant probes.

In another sense, by attempting to detect SM events of non-resonant probes, we are investigating the lowest detection limit of the SERS technique at the SM level, that is, the minimum observable cross-section that can be observed for a SM experiment using a standard Raman setup and SERS substrate. 
It is important to remember that non-resonant molecules represent the great majority of molecules of practical interest for applications. Indeed, most biological molecules and most chemicals in the environment are non-resonant. In terms of applying SERS to chemical detection, the lowest detection limit is obviously of great interest. As described in Section 2.4, a major impediment to the real-world application of SERS has always been the confusion relating to potential EFs, and consequently, there have been few attempts to give limiting values to the various substrates in terms of ultimate sensitivity in terms of minimum observable crosssection.

We believed that the field was still lacking a proper (and quantitative) demonstration of SM-SERS for non-resonant molecules and, by the same token, an assessment of the lowest possible SM detection limit achievable in practise. To this end, we explored the SM detection of non-resonant probes in an effort to quantify the minimum cross-section observable as a single molecule under standard SERS conditions. What we mean by standard Raman conditions is explored in the next section.

\subsubsection{Minimum cross-section measurable as a SM}

If we were able to integrate the signal of a single molecule for an unlimited amount of time $(\tau)$ and for an arbitrarily large laser power density $\left(I_{L}\right)$, we would eventually be able to obtain the signal above a minimum signal to noise ratio (SNR) and measure the spectrum of a single molecule without any enhancement. In the real world, however, intrinsic limitations exist, specific to the probe molecule, as to how much power density and integration time we can use. In fact, a more important parameter-as far as the photostability of the probe is concerned, i.e. its tendency to photobleach-is the product of the laser power density and the integration time $\left(I_{L} \times \tau\right)$. But limitations in integration time can arise by themselves without necessarily being linked to photobleaching. Liquid samples, for example, are limited by the diffusion time of the molecule spent in the scattering volume of the objective. Other instabilities of the signal (often categorized under the broad umbrella of "blinking") can exist. The latter comprise a whole variety of processes 
that go from molecular surface diffusion to subtle changes in the geometry of the SERS substrate [41, 49, 109]. Accordingly, having limitations in the maximum $I_{L}$ 's and $\tau$ 's we can use, there is an intrinsic limitation to how much signal we can obtain from a single molecule; the SERS enhancement then acts to bring this signal above the noise [110]. For the purposes here, when we refer to "characteristic values" of the enhancement needed to see single molecules, we are also implicitly assuming "characteristic experimental conditions" for real molecules as far as both $I_{L}$ and $\tau$ are concerned.

As stated, the limits of $I_{L}$ and $\tau$ are both system and analyte specific and must be determined experimentally. For solid substrates, in particular, signals arising from molecular degradation become overwhelming and this is usually below the maximum power of the laser (e.g. $\sim 5 \mathrm{~mW}$ for the $633 \mathrm{~nm}$ laser most commonly used). Additionally, because it is desirable to perform hundreds or thousands of acquisitions to give reliable statistics, there naturally arises a limit on the time per spectra (there are even limits to the available time of a graduate student!) As such, an integration time of anything more than about $30 \mathrm{sec}$ can become overly time consuming in terms of collecting useful amounts of data. In any case, other processes such as surface diffusion and photodegradation are likely to impose limits that keep us on the scale of seconds, rather than minutes. The characteristic values for measurement of a minimum cross-section for SM detection can thus be understood in this context.

\subsubsection{Previous reports}

It is important to highlight that claims of SM detection of non-resonant probes have been made in the past. For example, Refs. [111, 112] have claimed SM detection of adenine in SERS based on either "blinking" or "ultra-low concentration" approaches. The difference in the reliability of these latter techniques and a "contrast" method like BiASERS has been discussed in detail in the background material (Section 2.3.2; see also Refs. [41, 42, 43, 56]). 
For example, Ref. [111] makes no attempt to quantify the observed cross-sections but rather points out that blinking might be considered as evidence for SM sensitivity, while Ref. [112] works at ultra-low concentrations ( 30 pM) and derives estimates of cross-sections from vibrational pumping at room temperature, which as we have discussed (Section 2.5), must be taken with several provisos and are not a definitive measure, due mainly (but not exclusively) to laser heating effects and non-radiative contributions to the cross-section (further discussed in Ref. [72, 113]). Further evidence is given in the supposed existence of a Poisson distribution in the statistical analysis, which has also been shown to be an erroneous proof, not least because of the limited number of statistics (only 100 measurements) taken in that case [42].

Apart from SM detection, there are many studies of the trace detection of nonresonant molecules, most of which come from an applied focus, where the parameter of interest is usually the minimum measurable concentration of a given analyte $[114,115,116,117]$. This approach convolutes many different experimental factors, including the laser power and objective, the substrate EF and its distribution, along with the inherent properties of the probe. In this respect, it is difficult to compare among different systems. In contrast, by referencing our measurements to a reference standard and defining the EF appropriately, we are able to obtain a value, which although analyte specific and subject to optimization of conditions, is representative of the lowest detection limit of the substrate. 


\subsection{Method for SM detection of Non-Resonant Probes}

In many ways the time was ripe for a definitive demonstration of the SM detection of non-resonant probes. This thesis so far has outlined several important tools that can be used towards this goal. Critically, we have developed a rigorous methodology for identifying SM signals in SERS and for quantifying the EF to the best degree possible. Accordingly, the previous work has given us a good basis to extend the exploration of probes beyond those typically used in SERS, to those with low intrinsic Raman cross-sections.

The approach to SM detection essentially remains the same whether we are using resonant or non-resonant probes. However, in order to increase the integration time, we must turn to solid substrates, upon which (at least in principle) the molecule can be fixed in position; in reality, analyte-specific phenomena such as surface diffusion and adsorption/readsorption may limit this locatedness $[57,118,119]$.

Essentially, our methodology is the same as in previous chapters, outlined as follows:

(i) Determination of the non-SERS characteristics of the analyte of interest;

(ii) The use of BiASERS to unambiguously identify SM events;

(iii) Characterization of the objective on the day of experiments and measurement of a reference standard to allow calculation of the SMEF based on the peak intensity of the SM event; and

(iv) Where possible, the use of isotopic analogues as ideal partners for the $\mathrm{Bi}$ ASERS experiment.

Although the approach is effectively the same, in the case of non-resonant species we face a dramatic "change in scale" in terms of the experimental considerations. These include a much greater concern with practical issues such as contamination but also changes in fundamental aspects of the problem. 


\section{Shift in scale: sampling the upper region of the EF distribution}

The reduction in intrinsic cross-section of the molecule and the need for high EFs means we are now sampling the upper region of the EF distribution of our colloidal system; i.e. we are looking for molecules at the "hottest" hot-spots . The spatial distribution of the EF around hot-spots has been studied in detail for common colloidal systems $[42,43]$. In this section, we will briefly outline the basic elements of this discussion.

The probability $p(F)$ of having a certain SERS EF $F$ close to a hot-spot is always a tail-like distribution (with a cut-off), as schematically depicted in Fig. 7.1. The probability $p(F)$ will normally follow a power law of the type $\sim F^{-(1+k)}$ for the vast majority of gap hot-spots between nanoparticles, or surfaces and tips [110]. For typical plasmon gap resonances in silver or gold we have typical values of $k \sim 0.12-0.15$ [43] and a maximum "cut-off" enhancement of the order of $\sim 10^{11}$. This type of probability distribution, which has its origin in the extreme variations of the spatial distribution of the enhancements, is responsible for a big fraction of the phenomenology of SM-SERS statistics observed experimentally. Molecules with differential cross-sections of $\sim 10^{-27}-10^{-28} \mathrm{~cm}^{2} / \mathrm{sr}$ can be readily observed as single molecules around the $\sim 10^{8}$ enhancement range (in the sense explained previously, i.e. for characteristic values of $\left.I_{L} \times \tau\right)$.

Non-resonant molecules, on the other hand, with differential cross-sections of the order of $\sim 10^{-30}-10^{-31} \mathrm{~cm}^{2} / \mathrm{sr}$, require an additional boost of $\sim 10^{3}-10^{4}$ to the SERS enhancement in order to observe them as SM events. Thus, SM-SERS in this latter case can only come from the places with the largest enhancements in the distribution at about $\sim 10^{10}-10^{11}$.

This basic "change of scale" in the problem when going from resonant or preresonant molecules to non-resonant ones introduces a few peculiarities. From a purely electromagnetic point of view there is a much narrower range of enhancements that are "usable" at the very top of the distribution to push signals above the minimum SNR. Consequently, there is a much smaller effective area of hot-spots 


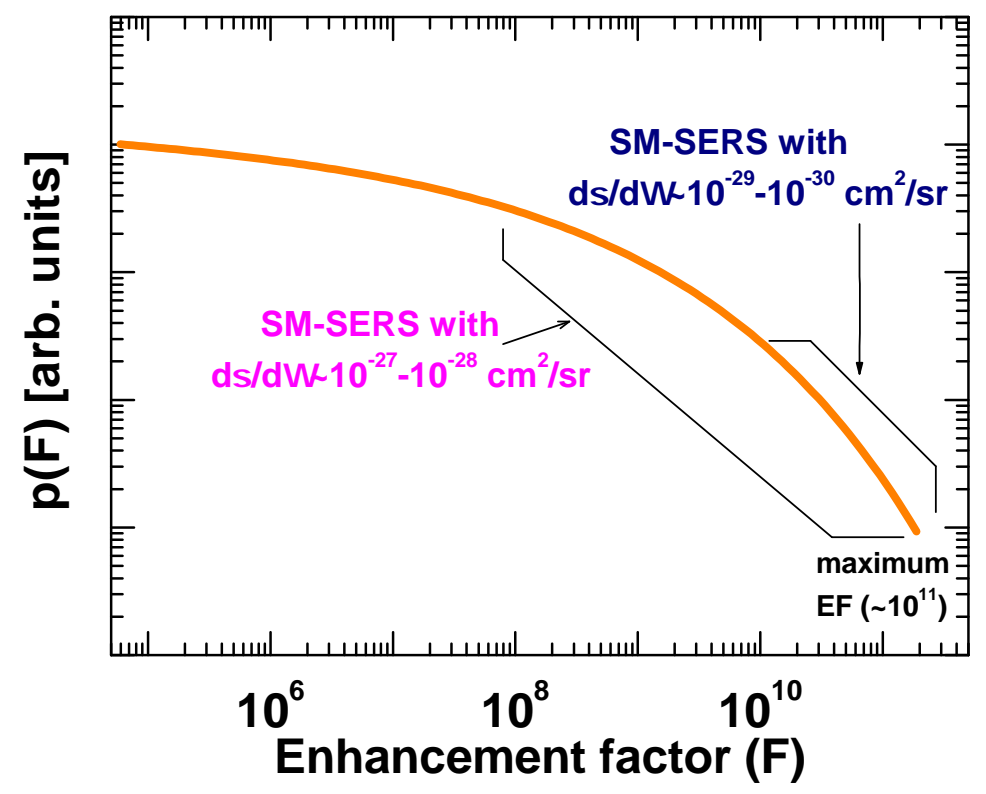

Figure 7.1: Typical long-tail probability distribution for the SERS EF on SERS substrates suitable for SM detection [30]. Molecules with differential cross-sections $10^{-27} \mathrm{~cm}^{2} / \mathrm{sr}$ are observable as single molecules above $F \sim 10^{8}$ for typical experimental conditions [56], while non-resonant molecules with $d \sigma / d \Omega \sim 10^{-30} \mathrm{~cm}^{2} / \mathrm{sr}$ require typical enhancements of $\sim 10^{11}$, at the high end of the distribution (and therefore of rare occurrence).

providing sufficient enhancement to produce a SM-SERS signal. Depending on the exact numbers we use, the effective area of hot-spots sufficient to observe single molecules with $d \sigma / d \Omega \approx 10^{-30} \mathrm{~cm}^{2} / \mathrm{sr}$ is $\sim 10^{2}$ times smaller than that for molecules with $d \sigma / d \Omega \approx 10^{-27} \mathrm{~cm}^{2} / \mathrm{sr}$. Accordingly, the statistics become much sparser. Expressed in a different manner: a much finer "tuning" of the conditions is required to see single molecules in this case. This covers only electromagnetic aspects of the problems, but there are additional experimental complications produced by the change in scale, which we expand in the next section. 


\section{General considerations}

One of the main problems for SM-SERS experiments on probes with cross-sections of the order of $d \sigma / d \Omega \approx 10^{-30} \mathrm{~cm}^{2} / \mathrm{sr}$ is that there are many other spurious competing molecules with similar (weak) differential cross-sections normally present in standard experimental conditions. This is particularly true for organic molecules because there are a myriad of spurious signals arising from organic moieties that are similar, or closely related to, the target molecule we are trying to monitor. In many cases, these spurious signals come from molecules present in the system via the synthetic route of the SERS substrate or as impurities. For resonant or pre-resonant dyes, this is not a problem because the dyes completely dominate the signal owing to their much higher intrinsic cross-sections. However, it does become an issue for non-resonant molecules. For example, in the standard Lee $\&$ Meisel colloid recipe [73], citrate molecules are used as a reducing/stabilizing agent [73]. When dried, these colloids are then left with an undesirable organic species on the surface with a cross-section comparable to the probe, thus complicating the analysis. Additionally, any inadvertent contaminants, particularly anything with a comparable or greater Raman cross-section, will also present a problem. Such factors mean careful optimization of the substrate and preparation conditions is required.

The problem, in fact, is more acute than the mere presence of undesirable organic species, because such organic molecules, particularly on dried substrates, are normally very susceptible to photodecomposition, thus creating "dynamic species" that change over time. The latter problem has been studied in some detail by several authors [118, 119], and leads ultimately (at high powers) to the well-known amorphous carbon-like spectral features (a "double dome" with humps at around $\sim 1350$ and $\sim 1600 \mathrm{~cm}^{-1}$ ). This amorphous carbon signal has a reported Raman cross-section four orders of magnitude higher than that of benzene and has been identified as one of the most difficult practical issues impeding widespread application of SERS of non-resonant molecules [22]. Interestingly, such signals do not appear to be such a problem for other metals such as gold and copper, suggesting it may be attributable to an aspect of the chemistry of silver [120]. 
For all the reasons described above, bi-analyte SERS (BiASERS) for non-resonant molecules is considerably more challenging than a mere "scaling" of the problem for resonant or pre-resonant probes. It means that a new dimension of problems have to be dealt with at what was basically the noise level for BiASERS of resonant or pre-resonant molecules.

\subsection{Experimental Details}

\subsubsection{Substrate optimization}

In an attempt to reduce contaminants arising from the substrate preparation we considered alternatives to the usual Lee \& Meisel colloids. Many initial measurements using these colloids gave rise to amorphous carbon-like signals. As an alternative to an organic reductant, we looked at colloids reduced with sodium borohydride (see Section 3.3.1 for details). In this reaction, borate $\left(\mathrm{BO}_{4}^{2-}\right)$ is the major byproduct, which as a non-carbonaceous species, would likely be less of a contaminant than the citrate stabilizer. Other literature reports also bear this out [121].

In terms of substrate preparation, a variety of different methods were trialled including drop casting, spin casting, spray coating, and methods involving deposition using alternative solvents to water. Most of these techniques gave equivalent results in terms of the level of spurious signals and decomposition. In fact, the best results were obtained using a relatively simple method involving repeated deposition of concentrated colloids under a heat gun. The results in this chapter apply to these substrates (preparation described in Section 3.3.3). 


\subsubsection{SERS experiments}

To prepare samples of the analytes for SERS experiments, a drop (50 $\mu \mathrm{L})$ of the analyte solution at low concentrations ( $25 \mathrm{nM}$ for all molecules presented here) was deposited onto the bare SERS substrate (prepared as described in Section 3.3.1) and left for $30 \mathrm{~min}$ in ambient conditions. Finally, the drop was sucked off with a glass pipette, and the sample rinsed in distilled $\mathrm{H}_{2} \mathrm{O}$ before final drying. This method was effective in preparing low concentration samples of the non-resonant analytes without noticeable contamination. The final surface coverage/concentration of the analyte cannot be determined with this method, but the SM nature of the SERS signals can be assessed with the BiASERS technique, and the concentration adjusted if necessary.

\subsubsection{Linear deconvolution analysis}

As has been discussed, the identification of SM signals of non-resonant probes is not as straightforward as for resonant or pre-resonant probes, due to the fact that other spurious signals from contaminants or photodegradation compete with the probe of interest. For this reason, techniques such as the Modified Principal Component Analysis (MPCA) method [52] (described in Appendix A.6.3) are not possible in general (because the principal components of the problem are not well defined in a background of widely changing signals).

This problem can be circumvented by performing a fitting routine to the data which deconvolutes the BiASERS spectra into a linear combination of the contributions from experimentally obtained reference spectra of the individual molecules along with a linear background (see Appendix A.6.2) The validity of the fit (or "goodness-of-fit" value) is then determined by a $\chi^{2}$-parameter, which is normalized to the spectral intensity of the event. Fits below a certain intensity (below noise level) and those with a "goodness-of-fit" below an appropriately chosen threshold are discarded along with any remaining non-physical fits. This fitting process, in combination with suitable selection criteria, can effectively discard erroneous events arising from photodegradation and contaminants, and ultimately 


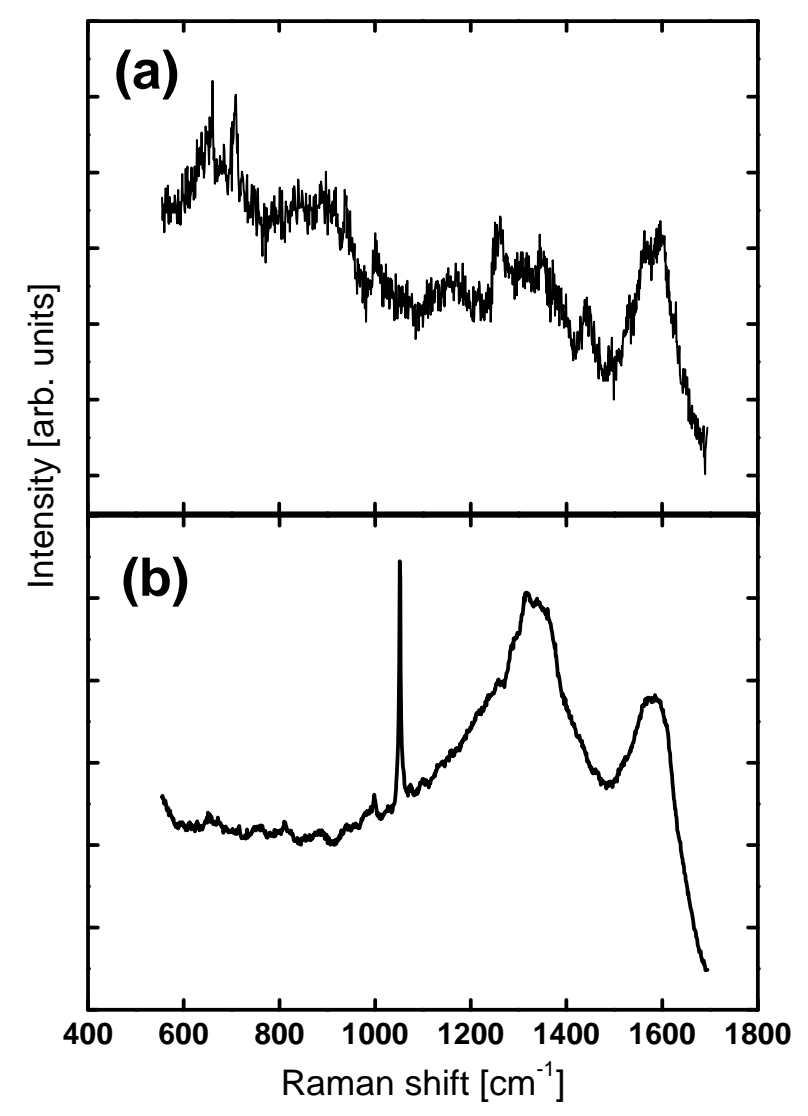

Figure 7.2: (a) An example of typical spurious spectra observed frequently during maps. (b) Characteristic double hump of amorphous carbon-like features resulting from photodegradation.

recover the statistics of the two molecules of interest.

\subsubsection{Optimizing laser conditions}

As pointed out in the introduction, we are often limited [119] in the maximum amount of (laser) power we can deliver to the sample, particularly with a dried substrate, by photo-induced degradation on a sub-sec time scale. The observation of photoproducts is particularly a problem for resonant of pre-resonant molecules if the photo-stability of the probe is poor (which is the case, for example, of dyes like malachite green [97]). But perhaps less intuitively, photo-degradation in SERS experiments remain an issue for non-resonant molecules [121, 122, 123]. 
Reference [122] shows explicitly how the photochemistry of degradation of aromatic non-resonant species evolves in time, resulting eventually-after some transient intermediate states-into a final photo-product that is similar to amorphous carbon in its spectral characteristics (but with some of the details being determined by the exact precursor). At high power densities ( $\sim 5 \mathrm{~mW}$ focused onto a $1.4 \mu \mathrm{m}$ waist beam by the $\times 50$ air objective), such signals were indeed observed for many of the analytes tested. At this power level transient photoproducts are seen on a sub-sec time scale with randomly occurring peaks in the range characteristic of typical organic moieties with $s p^{2}-s p^{3}$-hybridizations of carbon, with a spectrum tending toward "amorphous-carbon-like" for long expositions to the laser on the same spot. Figure 7.2 gives examples of a random erroneous spectrum (a), and the characteristic spectrum for amorphous carbon (b).

\subsubsection{Choice of the non-resonant probes}

In terms of the selection of appropriate probes, it would be desirable to find molecules that have commercially available isotopic analogues so that we can perform BiASERS with chemically identical probes (as outlined in Chapter 6). Having the probes commercially available would then allow any researcher to purchase the relevant molecules and perform SM experiments with the expectation that SM detection of the probes would be a valid guide to the fundamental limits of their Raman system and SERS substrate. Again, the isotopic partners need to have distinct spectral features for separate identification in BiASERS. For this purpose, DFT calculations are an invaluable guide, in that they give us an indication of the degree of spectral distinction between the analogues. Additionally, they provide us with an estimate of the bare Raman cross-section (Section 2.1.6). The experimental details for these calculations are given in Section 3.4.5. 


\subsubsection{Initial experimental attempts}

Several systems were explored for some plausible candidate probes known to have commercially available isotopic analogues. After using DFT to confirm spectral variation (or the potential for spectral variation given the limitations of DFT), the natural version of the probe was first measured in a standard SERS experiment at a variety of concentrations to see whether it could be detected at low levels. Table 7.1 gives a summary of the probes tested at various concentrations and using several different substrate preparations. For various reasons, the initial probes did not give the SERS signals hoped for and it was not possible to unambiguously identify the molecule at low concentrations. Although some time was spent on each probe to optimize conditions, such as varying the $\mathrm{pH}$, laser powers and integration times, it was decided that a sensible approach forward would be to first identify probes that, although non-resonant, have larger intrinsic cross-sections (up to $\sim 10^{-29} \mathrm{~cm}^{2} / \mathrm{sr}$ ) than the typical non-resonant molecules attempted so far ( 10 $\left.10^{-31}-10^{-32} \mathrm{~cm}^{2} / \mathrm{sr}\right)$. If we could demonstrate success with such a system, we could then explore the lower limits on a firmer footing. 
Table 7.1: Initial non-resonant probes tested in SM experiments. Despite attempts to optimize the substrate preparation and SERS conditions, we had minimal success in identifying a characteristic signal above spurious events. This may be an indication that the probes are photochemically unstable.

\begin{tabular}{|l|l|}
\hline Probe & Structure \\
\hline Mercaptoethanol & Promethazine \\
\hline Chloro-mercaptobenzoxazole
\end{tabular}

\subsection{Measurement of BPE/BTZ System}

We next looked to find a non-resonant probe with an intermediate cross-section in the range $\sim 10^{-29} \mathrm{~cm}^{2} / \mathrm{sr}$. After exploring several different probes, we selected 1,2-di-(4-pyridyl)-ethylene (BPE; obtained from Sigma-Aldrich). BPE is known to attach efficiently to SERS substrates and has been used repeatedly in Raman [124] and SERS experiments in the past [125, 126, 127]. DFT calculations suggested vibrational modes with cross-sections on the order of $\sim 10^{-29} \mathrm{~cm}^{2} / \mathrm{sr}$.

As it was not possible to commercially obtain an isotopic analogue in this case, and with synthesis likely to be an involved and time-consuming process, we looked to other molecules which we had previous experience with as a BiASERS partner to BPE $[41,56]$. To this end, we decided to use the benzotriazole dye measured 
(a)<smiles>C(=C/c1ccncc1)\c1ccncc1</smiles>

1,2-di-(4-pyridyl)-ethylene (BPE) (b)

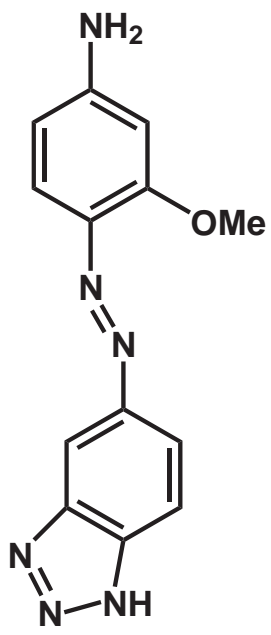

(3-methoxy-4-(5'-azobenzotriazolyl) phenylamine (BTZ)

Figure 7.3: Chemical structures of BPE (a) and BTZ (b).

in Chapter 4, 3-methoxy-4-(5'-azobenzotriazolyl) phenylamine (dye no. 2 of Ref. [88], denoted BTZ). The measured cross-sections of this dye are on the order of $10^{-28} \mathrm{~cm}^{2} / \mathrm{sr}$ (Table 4.4) and its characterization in SERS is given in Chapter 5.

\subsubsection{Basic properties of probes}

Importantly, BPE and BTZ have distinct spectral features for separate identification, and similar bare Raman cross-sections. Before measurement in SERS, the first step is to fully characterize the bare molecules.

The UV-Vis absorption spectra of the two molecules are shown in Fig. 7.4. Both molecules are non-resonant at $633 \mathrm{~nm}$ excitation, with BTZ absorbing close to the $\operatorname{UV}(\lambda=427 \mathrm{~nm})$ and BPE in the $\operatorname{UV}(\lambda=300 \mathrm{~nm})$. One could argue that BTZ still exhibits a small pre-resonant Raman effect at $633 \mathrm{~nm}$, and this is indeed supported by its differential Raman cross-section of $\sim 10^{-28} \mathrm{~cm}^{2} / \mathrm{sr}$ in water (Table 4.4). However, it is important to recognize that BTZ is used here 


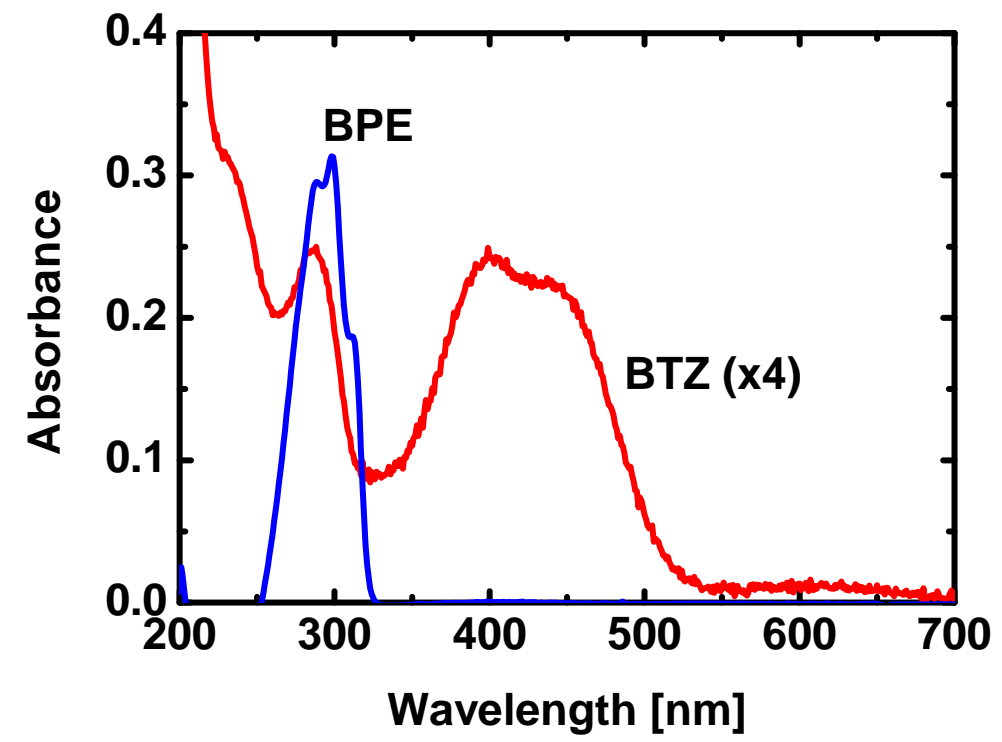

Figure 7.4: UV/Vis spectra of BPE and BTZ molecules [88] taken at $10 \mu \mathrm{M}$ concentration. BPE was measured in ethanol because of its limited solubility in water. Both molecules are non-resonant at $633 \mathrm{~nm}$ laser excitation.

as a convenient and well-understood BiASERS partner to BPE, rather than as an example of a non-resonant probe.

\subsubsection{Measurement of bare Raman cross-sections}

For all the experiments, the standard setup (Section 3.1) was used with a $633 \mathrm{~nm}$ HeNe laser. For the measurement of bare Raman cross-sections of BPE, a $\times 100$ Olympus water immersion objective (N.A. 1.0) was used, and Raman cross-sections were estimated with respect to the $516 \mathrm{~cm}^{-1}$ peak of the reference $2 \mathrm{~B} 2 \mathrm{MP}$ as described in Chapter 4. Because BPE is only sparingly soluble in water, BPE was measured in a solution of ethanol $(1 \mathrm{mM})$. Immediately following measurement a background signal of pure ethanol was measured under the same conditions and in this way a BPE spectrum was obtained with the background removed. Serendipitously, most of the BPE peaks were in different positions than the ethanol peaks, which would swamp any other peaks in the immediate vicinity owing to the much 
greater concentration of ethanol than BPE. Cross-sections of BTZ had already been measured, as given in Chapter 4.

For the SERS experiments on solid substrates, a $\times 50$ Olympus long workingdistance objective (N.A. 0.5) was used, and X-Y maps were taken of selected areas to give a large statistical sample of events. SMEFs were obtained after a careful characterization of the optical setup on the day of the experiments, as described in previous chapters. The scattering volume of the objective was estimated by measuring the axial and confocal pinhole dependence of the intensity on a $\mathrm{Si}$ wafer (given in Appendices A.4.2, A.4.3). The measured scattering volume of the $\times 50$ objective (N.A.=0.5), was found by this method to be $V=152 \mu \mathrm{m}^{3}$ (Table 3.1). The SM signals, as identified by the BiASERS procedure, were calibrated with respect to the signal of the $2330 \mathrm{~cm}^{-1}$ mode of nitrogen at room temperature and standard pressure (using long integration times).

\subsection{Results and Discussion for BTZ/BPE System}

\subsubsection{BiASERS experiments}

After preparing the dried samples, we performed a series of experiments to determine suitable conditions for laser power and integration time. We found that a reasonable power to use was $\sim 0.5 \mathrm{~mW}$ focused onto a $1.4 \mu \mathrm{m}$ diameter beam by the $\times 50$ air objective, which produced signals stable for $\sim 1 \mathrm{sec}$ without an overwhelming proportion of events with obvious signatures of photo-degradation. These parameters then fix the minimum differential cross-section that can be observed in this case, as has been discussed. All SERS maps were thus taken under these laser conditions for moderate integration times of $1 \mathrm{sec}$.

BiASERS maps were then taken of the prepared substrates. The window selected for fitting was quite small (1580 to $\left.1680 \mathrm{~cm}^{-1}\right)$, because the BPE and BTZ peaks in this region, although partially overlapping, were sufficiently distinct to allow adequate deconvolution while allowing for any slight shift in wavenumber (up to $\sim 3 \mathrm{~cm}^{-1}$ ). 


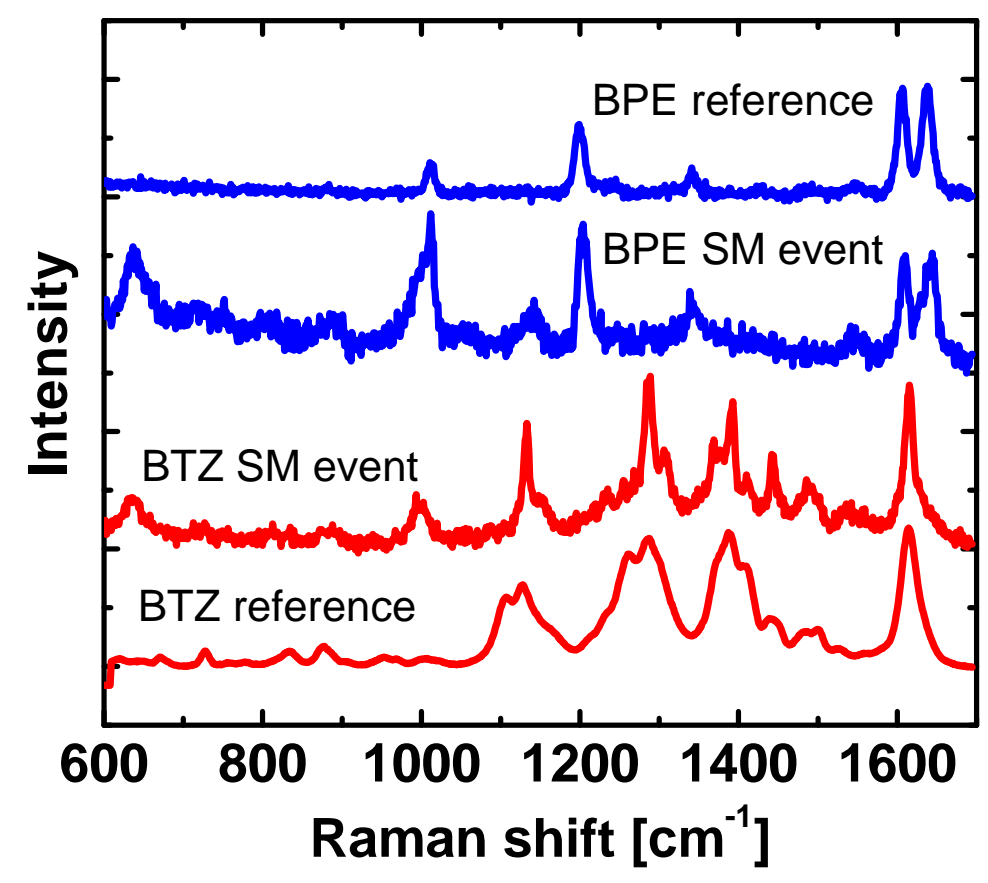

Figure 7.5: Example of individual SM-SERS events of BPE and BTZ along with average SERS spectra obtained by measurement of each analyte separately. From these SM-SERS events, and a careful characterization of the optical setup [56] and bare differential cross-sections, the SMEFs are then obtained. The spectral features at $\sim 633,1100$, and $1550 \mathrm{~cm}^{-1}$ in the SM-SERS events are also observed in spectra of the bare SERS substrate and do not belong to BTZ or BPE.

From 4900 spectra, it was possible to identify 326 events that passed the noise and "goodness of fit" thresholds. Figure 7.5 shows examples of the SM spectra of BPE and BTZ, along with the (reference) average SERS spectra of each molecule obtained individually. For the BTZ SM event, it can be seen that the spectral resolution of the vibrational modes is somewhat narrower for the SM event than for the reference spectra. BTZ Raman peaks are almost all doublets and this narrowing may therefore be attributed to different relative intensities within a doublet (as a result of surface selection rules, for example). Studies by other authors [57] have also suggested that such narrowing is a characteristic of SM spectra; however, the exact origin of the linewidths has to be analyzed in general with uttermost care 
(because it can include instrumental components, like the exact position of the image on the entrance slit of the spectrometer for different events). We make no attempt here to interpret the linewidths, but rather focus on the integrated intensity of the peaks. Figure 7.6 shows the corresponding intensity histogram of these events, where the abscissa shows the relative contributions of each reference spectra to the BiASERS spectra. From the histogram, it can be seen that there are 79 SM events of BPE (right side of the histogram) and 66 of BTZ (left side of the histogram) in the map.

It is worth noting that these numbers do not necessarily have the normal statistical meaning as in the standard BiASERS of resonant or pre-resonant probes, for there are many signals (of both types) that are not actually counted because they are contaminated with spurious photo-product signals, i.e., peaks that belong to unidentified carbonaceous species [122]. On the contrary, these numbers represent the random chances of finding a "clean" signal in a background of spurious (and comparable in size) peaks. Notably, the chances of finding each analyte as a single molecule are equivalent, and situations in between are randomly distributed. This indicates that we are effectively entering into the SM regime as defined by the BiASERS technique [52].

It also became apparent after the conclusion of this work, that there may be complications with both the BPE and BTZ probes, in that there may be the possibility of them undergoing photoisomerization around the double bonds, resulting in the existence of both cis and trans versions. For example, there exist several experimental and theoretical studies on the photoisomerization of azobenzene $[128,129,130]$, which represents the 'core' of the BTZ molecule. One study showed isomerization of the trans form to the cis form at a wavelength of $450 \mathrm{~nm}$ with a quantum yield of 0.25 [128]. A recent article has shown the observation of isomerization of an azobenzene derivative in a SERS experiment [131], although it should be emphasized that these experiments were conducted in the near-UV (at $365 \mathrm{~nm}$ ). In our case, while the possibility of photoisomerization can not be excluded for the particular BTZ molecule under study, there were no signs in the experiments that it was a problem, and it is expected given the results for azobenzene that excitation at $633 \mathrm{~nm}$ is too low to allow photoconversion, particularly as 

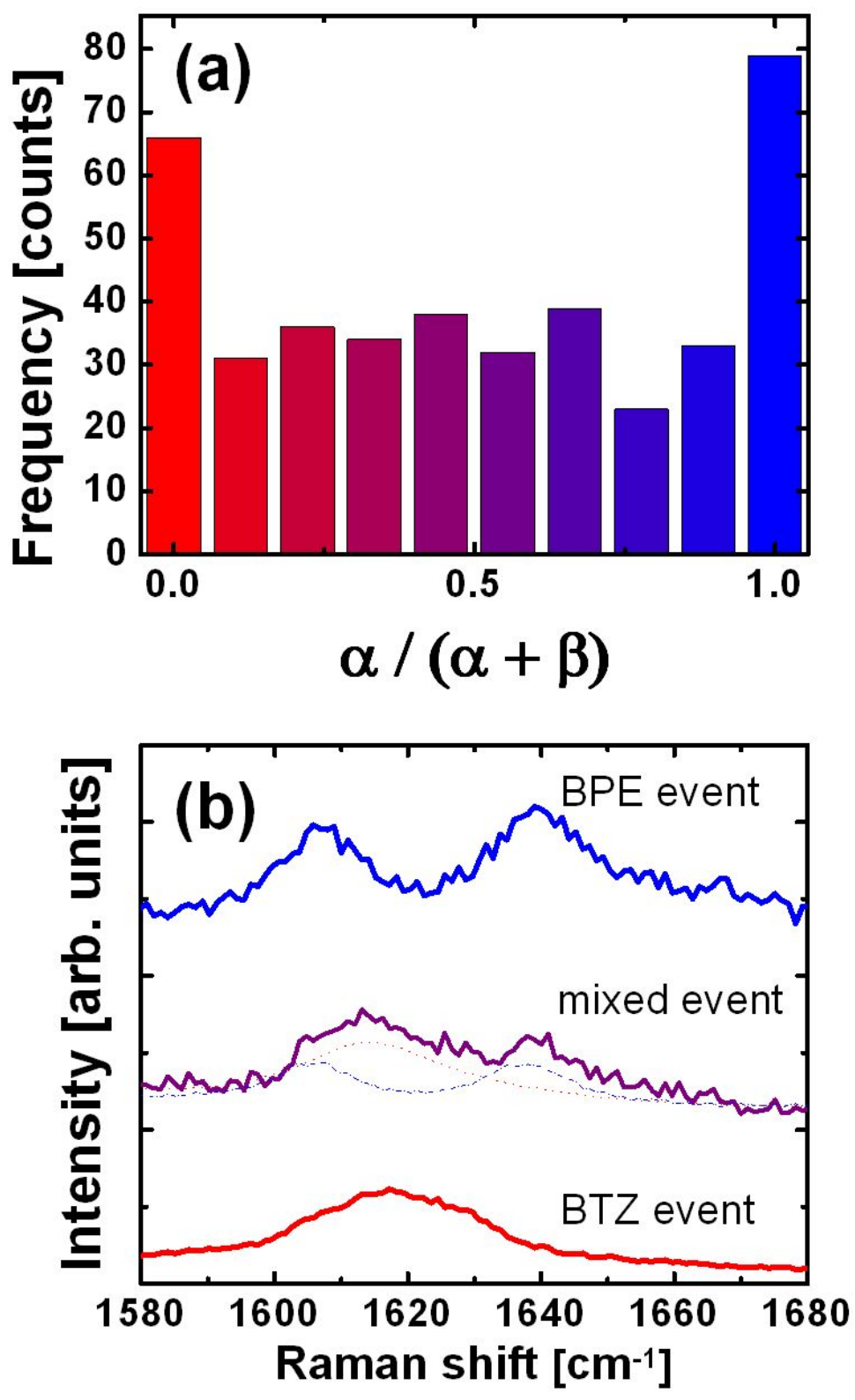

Figure 7.6: (a) Histogram showing relative proportions of BPE and BTZ reference spectra for valid BiASERS spectra. The abscissa $(\alpha /(\alpha+\beta))$ represents the fraction contributed by BPE (i.e. $\alpha /(\alpha+\beta)=0$ is "pure BTZ", $\alpha /(\alpha+\beta)=1$ is "pure BPE"). The relatively large number of SM events of either molecule indicates we are entering the SM regime [52]. (b) Three representative cases of a pure BTZ or BPE spectrum, along with a mixed spectrum. Linear deconvolution is for the $1580-1680 \mathrm{~cm}^{-1}$ range, which contains distinguishable Raman fingerprints for both dyes. Details on linear deconvolution are given in Appendix A.6.2. 
it is thought that the isomerization is mediated via electronic transition.

\subsubsection{SMEFs for BPE}

Having identified SM signals, we can now obtain SMEFs following characterization of the objective and measurement of the $2331 \mathrm{~cm}^{-1}$ mode of $\mathrm{N}_{2}$ (see Appendix A.5.1 for details). There is obviously no single SMEF that represents all SM-events, but rather a distribution of SMEFs for single molecules in different conditions. From the range of SM events observed, we focus on the maximum EFs, i.e. those estimated from the first few largest SM-SERS events (examples of which are shown in 7.5).

The results are summarized in 7.2. Here, we obtain maximum SMEFs in the range of $1-5 \times 10^{10}$ for both BTZ and BPE. Notably, however, there were also SM events detected with smaller EFs, down to $\sim 5 \times 10^{9}$ for the non-resonant molecule BPE. Being able to see these SM events at EFs below the maximum achievable EFs for our system suggests the possibility of observing non-resonant molecules with even lower intrinsic cross-sections (by a factor of $\sim 5-10$ ).

It is important to realize that these values come from: $(i)$ independent characterization of the bare probes, which implies a full calibration with respect to a reference in liquid (2B2MP); and (ii) comparison of the SM-SERS cross-section with respect to another reference (nitrogen gas). The fact that all these determinations can be put together to produce a number which is perfectly in agreement with the expectations of maximum EFs at hot-spots is quite remarkable in itself, and it shows a pleasing consistency of the underlying physics of the effect, which has been for many years elusive due to the uncertainty in the number of molecules being measured. This is the specific aspect that the BiASERS technique solves, thus enabling quantitative comparisons that would not be feasible otherwise.

It is also worth noting the small discrepancy in maximum SMEFs measured for different Raman modes of the same molecule or between those of BTZ and BPE. One obvious interpretation for the latter is the presence of a small additional chemical enhancement (of the order of $\sim 2-4$ ) for BPE. However, other mechanisms 
could also cause this small discrepancy, including:

(i) BTZ could be more susceptible to photo-degradation than BPE, and although not observable as distinctive amorphous carbon features in the SERS spectra, it is possible that the SMEFs of BTZ are limited in the highest EF cases by photo-degradation within the integration time; and

(ii) surface selection rules $[2,94,132]$ could also result in small discrepancies of this order between Raman modes of a molecule or between two different analytes.

For example, the SMEFs among the different modes of BTZ in the present case (Table 7.2) differ in relative terms from the AEFs obtained for this molecule in Chapter 5 (Table 6.4), indicating the possibility of surface-selection rules playing a role. In this case, a specific mode may be enhanced for individual molecules bound in a particular position, with this effect being washed out in the average situation, which the AEF measures. The relatively poor peak fits for this molecule prohibit definitive conclusions in this respect, however. It is not possible to conclude from our results here which mechanism (or combination of them) was at play, and as this was not the object of the study we did not explore these differences further.

Finally, these EFs are generally larger than those obtained for the SM signals obtained in Chapter 5 (Figs. 5.4 and 5.5). In an analogous manner to the anthropic principle, this is simply a consequence of the "need" to have such EFs to see the signals in the first place. In practise, the necessary experimental optimization and troubleshooting to find a SM event in this case is much harder than for resonant probes. 
Table 7.2: Main Raman active modes of BPE (in ethanol) and BTZ (in water). Also shown are the corresponding typical maximum SMEFs, representative of the largest SMSERS events identified with the BiASERS method. Note that the results for BTZ are much more uncertain because most Raman/SERS peaks are relatively wide and part of a doublet. In the case of BPE, a single event exhibited SMEFs $\sim 50 \%$ larger than those shown here, but it was excluded from the analysis because of the lack of statistics on such rare events.

\begin{tabular}{lllll}
\hline \hline & $\begin{array}{l}\overline{v_{i}} \text { (Raman) } \\
{\left[\mathrm{cm}^{-1}\right]}\end{array}$ & $\begin{array}{l}\text { bare } \frac{d \sigma}{d \Omega} \\
{[\mathrm{cm} 2 / \mathrm{sr}]}\end{array}$ & $\begin{array}{l}\overline{\bar{v}_{i}} \text { (SERS) } \\
{\left[\mathrm{cm}^{-1}\right]}\end{array}$ & $\begin{array}{l}\text { SMEF } \\
-\end{array}$ \\
\hline BPE & 1001 & $2.6 \times 10^{-29}$ & 1010 & $4.2 \times 10^{10}$ \\
& 1197 & 2.1 & 1202 & 4.8 \\
& 1343 & 1.4 & 1342 & 1.9 \\
& 1601 & 2.9 & 1608 & 3.4 \\
& 1641 & 5.5 & 1639 & 3.2 \\
\hline BTZ & 1108 & $1.0 \times 10^{-28}$ & 1134 & $2 \times 10^{10}$ \\
& 1412 & 2.5 & 1410 & 1 \\
& 1617 & 0.87 & 1617 & 1 \\
\hline \hline
\end{tabular}

\subsection{Results and Discussion for Adenine System}

The successful SM detection of the non-resonant probe BPE renewed our attempts to detect probes with even lower intrinsic cross-sections (down to $\sim 10^{-30} \mathrm{~cm}^{2} / \mathrm{sr}$ ). It would also be preferable, for the many reasons outlined in Chapter 6, to return to the methodology of isotopically edited probes. The use of isotopically edited partners in combination with the BiASERS technique is arguably the ideal system for exploring SM detection as both probes have the same chemical characteristics in terms of surface adsorption and resonance properties [57, 133]. For these investigations, the DNA nucleotide base adenine was selected as the initial probe because: (i) its SERS properties are well characterized [111, 112, 134]; (ii) it contains nitrogen groups suitable for binding with the silver surface; (iii) it has biological relevance; and (iv) it has a commercially available isotopic analogue. Subsequent DFT calculations gave estimates for the Raman cross-sections on the 
<smiles>Nc1ncnc2[nH]cnc12</smiles>

adenine<smiles>Nc1nc[nH+]c2[nH]cnc12</smiles>

${ }^{15} \mathrm{~N}$-adenine

Figure 7.7: Chemical structures of adenine and ${ }^{15} \mathrm{~N}$-adenine.

order of $\sim 10^{-30} \mathrm{~cm}^{2} / \mathrm{sr}$. Historically, as outlined in Section 7.1.2, adenine was used in the first attempts at SM detection of non-resonant probes [112]. We believe, however, that our approach overcomes the concerns identified with the initial proof of SM detection and therefore represents the first unambiguous proof of SM detection of adenine.

As a BiASERS partner to adenine, we used an isotopically substituted adenine, $\left({ }^{15} \mathrm{~N}\right)_{2}$-1,3-adenine (henceforth referred to as ${ }^{15} \mathrm{~N}$-adenine, purchased from Isotec, Sigma-Aldrich), for which the Raman/SERS spectrum is sufficiently different from (natural) ${ }^{14} \mathrm{~N}$-adenine to distinguish them in a BiASERS experiment (see Fig. 7.7).

\subsubsection{BiASERS and SMEFs for adenine}

We prepared substrates using borohydride colloids deposited on silicon in an identical manner as for the BPE/BTZ system (Section 3.3.3). A map of the substrate was taken with a large number of spectra (5535) and SM events were identified by linear deconvolution of adenine and ${ }^{15} \mathrm{~N}$-adenine reference spectra measured individually, along with a linear background. The reduction in intrinsic cross-section was immediately noticeable, with a much greater proportion of events dominated by spurious signals, either from contaminants or photo-degradation processes. For this reason, it was not possible to find as many SM events as with the other probes. Nonetheless, several examples of SM-events of each molecule could be isolated (at the expense of a much larger sampling) as shown in Fig. 7.8. In this case, it 
Table 7.3: Main Raman active modes of adenine and ${ }^{15} \mathrm{~N}$-adenine (in water) along with their experimentally determined non-SERS cross-sections. Also shown are the corresponding typical maximum SMEFs, representative of the largest SM-SERS events identified with the BiASERS method.

\begin{tabular}{lllll}
\hline \hline & $\begin{array}{l}\bar{v}_{i}^{(\text {Raman })} \\
{\left[\mathrm{cm}^{-1}\right]}\end{array}$ & $\begin{array}{l}\text { bare } \frac{d \sigma}{d \Omega} \\
{\left[\mathrm{cm}^{2} / \mathrm{sr}\right]}\end{array}$ & $\begin{array}{l}\bar{v}_{i}^{(\text {SERS })} \\
{\left[\mathrm{cm}^{-1}\right]}\end{array}$ & SMEF \\
\hline adenine & 724 & $2.9 \times 10^{-30}$ & 735 & $7.1 \times 10^{10}$ \\
${ }^{15} \mathrm{~N}$-adenine & 716 & 3.2 & 728 & 10.2 \\
\hline \hline
\end{tabular}

was not possible to obtain SMEFs for most of the modes because individual peaks were difficult to resolve in the SM-SERS spectra. It was possible, however, to get good fits for the peak at $\sim 735 \mathrm{~cm}^{-1}$ for adenine $\left(\sim 728 \mathrm{~cm}^{-1}\right.$ for ${ }^{15} \mathrm{~N}$-adenine $)$ and to obtain representative maximum SMEFs for these modes (see Table 7.3). These maximum SMEFs, at $7 \times 10^{10}$ and $1 \times 10^{11}$, are consistent with the BPE results, and represent the upper end of available EFs for this system. In the case of adenine, these are also the minimum SMEFs for which SM-SERS events could be detected.

\subsection{Conclusions}

Maximum EFs in the range $\sim 10^{10}-10^{11}$ are perfectly within reach of classical electromagnetic theory of gap plasmon resonances [2, 28, 29, 43, 110, 135, 136]. Like many situations in SERS, it is conceivable that this number has a small contribution from a "chemical enhancement" [137]. The observed frequency shifts are sometimes an indication that a small chemical component of the enhancement might be present through the interaction of the molecule with the substrate. But it is not the main topic here to separate the relative contributions of the "electromagnetic" and "chemical" enhancements (which we cannot separate unambiguously in any case) but rather to point out that EFs of $\sim 10^{10}-10^{11}$ are sufficient to see single molecules with some of the smallest differential cross-sections. Accordingly, these results demonstrate that it is possible to detect non-resonant probes 


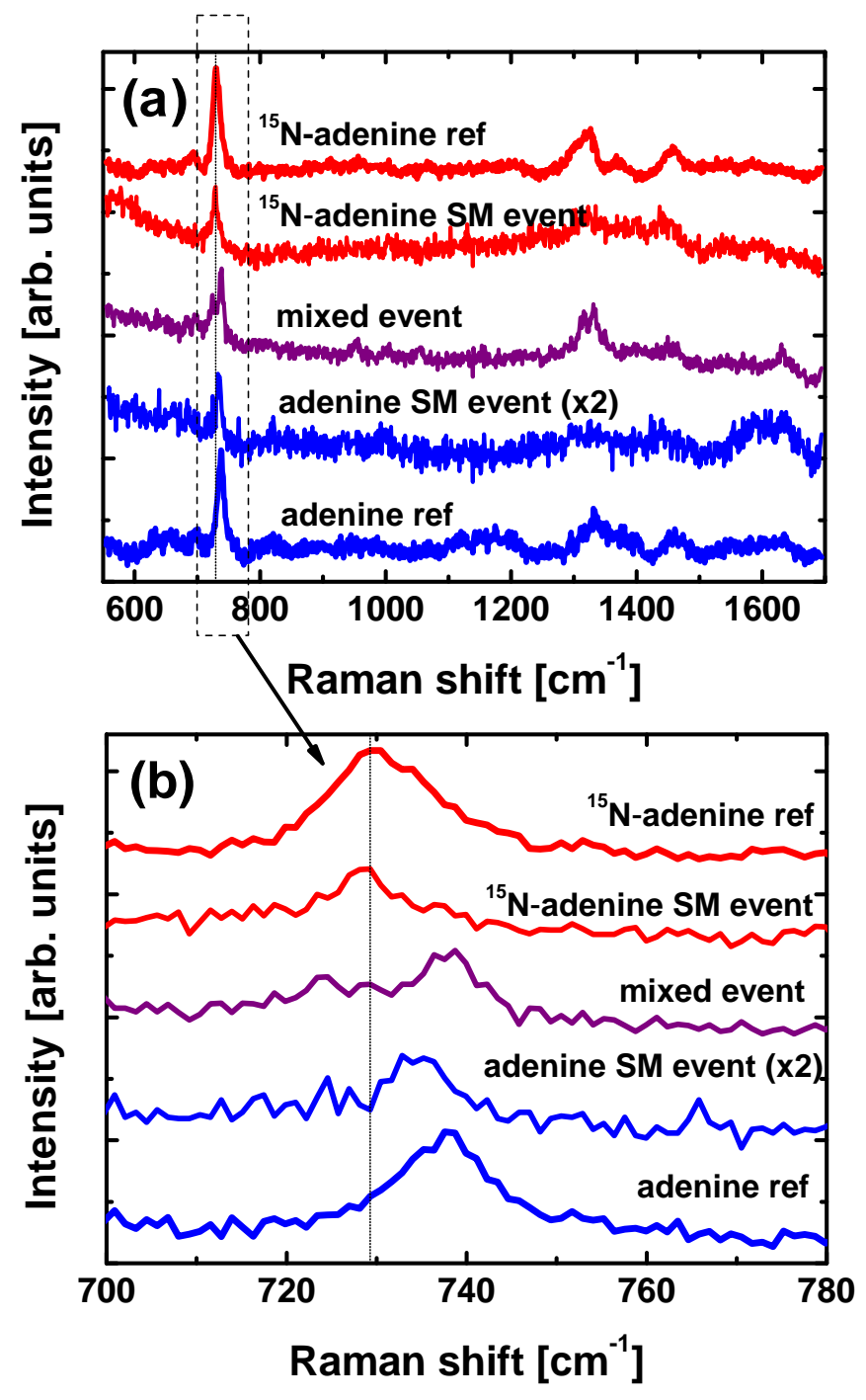

Figure 7.8: (a) SM events of adenine and ${ }^{15} \mathrm{~N}$-adenine along with a mixed event identified from a BiASERS map. The reference average SERS spectra of each molecule are also shown. The adenine $/{ }^{15} \mathrm{~N}$-adenine BiASERS experiment was performed using the same substrate preparation and experimental conditions as the BTZ/BPE results. (b) Expansion of the $700-780 \mathrm{~cm}^{-1}$ range for the spectra in (a). This was also the range used in performing linear deconvolution of the BiASERS spectra to analyse the SM events. 
with intrinsic Raman cross-sections down to $\sim 10^{-30} \mathrm{~cm}^{2} / \mathrm{sr}$. In that sense (as pointed out in the introduction), we see the results presented in this chapter as a fundamental proof of principle of the minimum yardstick detection level of the technique at the SM level.

Interestingly, a study by Gu et al. on the non-resonant molecule $p$-aminothiophenol resulted in an estimate for the minimum EF to see non-resonant molecules of $\sim 10^{11}$. This value was estimated by linear extrapolation to the SM regime based on a range of low concentration measurements $(0.5-6 \mathrm{nM})$, and an estimate of the number of molecules adsorbed on the SERS substrate at these concentrations [138], so the value is necessarily dependent on their underlying assumptions. Nonetheless, their estimate is certainly consistent with our results.

As seen with the initial failures (Table 7.1), the possibility of doing SM-SERS with other non-resonant molecules of interest can only be decided on a case-bycase basis, depending on the stability of the molecules under given experimental conditions, and the likelihood of spurious "carbonaceous" species of comparable weak cross-sections (plus additional possible problems with photo-stability). However, we have shown here that with the right preparation conditions, and suitable selection of experimental conditions, it is indeed possible to see SM events of non-resonant molecules. The conditions involve an inevitable compromise between laser power, integration time, and sample preparation.

The results presented here show that, under the right conditions, differential crosssections of the order of $\sim 10^{-30} \mathrm{~cm}^{2} / \mathrm{sr}$ are indeed measurable, and this would be approaching the lowest detection limit of the technique at the SM level. As many of the molecules of widespread interest fall into this category, this is an important result in terms of applications. If all experimental problems are overcome, we are forced to conclude that in principle any molecule adsorbed on a substrate can be observed at the SM level with SERS. This is a statement that has been made several times in the literature in different contexts $[139,140]$, but never with a 
proper justification through a specific quantitative example. We believe the results presented in this thesis provide a conclusive demonstration of SM-SERS for nonresonant probes, and set the lowest detection limit of SM-SERS on a firm footing. 


\section{Chapter 8}

\section{CONCLUSIONS}

This thesis has outlined a rigorous and stepwise process towards the accurate quantification of the SERS enhancement factor (EF).

The final estimates of the EF, we believe, represent the most accurate evaluations of EFs to date and refute the massive values reported in the literature of up to $10^{14}[40,62]$. Such values have been based on inappropriate normalization of the EF, which does not take into account the fact that some molecules can have much larger intrinsic Raman cross-sections than may be assumed. For example, the cross-section of common dyes such as crystal violet and rhodamine are several orders of magnitude greater than the non-resonant molecule methanol because of resonance or pre-resonance effects. As a result, the bare Raman cross-section must always be measured, if fluorescence does not limit its measurement.

In obtaining the EFs, a key part of our methodology was the use of an appropriate reference with a known cross-section. The selected reference, 2-bromo-2methylpropane (2B2MP) was shown to be ideal in terms of reliability and practical convenience, and is expected to play a useful role as a reference standard in future SERS experiments. Although a simple methodology, this is the first example of the use of a reference compound for the estimation of Raman cross-sections in SERS. In summary, the main elements of our approach are as follows: 
(i) Careful consideration of the appropriate EF definition;

(ii) Correct normalization of the SERS EF to the bare Raman cross-section;

(iii) Use of a reference standard to calculate bare Raman and SERS cross-sections;

(iv) Beam profiling experiments to characterize the scattering volume or excitation;

(v) The use of a liquid reference (2B2MP) or a gas reference $\left(\mathrm{N}_{2}\right)$ to calibrate the signal;

(vi) The use of bi-analyte SERS (BiASERS) to unambiguously identify SM events;

(vii) The complementary use of BiASERS along with temperature dependent vibrational pumping (TDVP) for obtaining the SERS cross-section and single molecule enhancement factor (SMEF); and

(viii) The extension of BiASERS to isotopically edited partner molecules.

It is notable that for a large proportion of the EFs quoted in the literature, very few of these steps are performed. Instead, standard assumptions are made about monolayer coverage, typical packing densities, and the characteristic values of the objective may only be based on manufacturer guidelines. As such, the present work represents a fundamental improvement in the rigour of the experiments, providing as a result much greater accuracy.

In the work of Chapter 5, the analytical enhancement factor (AEF) emerged as a convenient measure of the EF for a given system prepared under identical conditions. Notably, the AEFs for five considerably different molecules were all on the order of $\sim 10^{5}$, indicating that, in this case, chemical enhancement did not play a signficant role (if we exclude the unlikely possibility of five such different probes having identical chemical enhancements). This result demonstrates the value in accurately measuring the EF in yielding insights into the fundamentals of SERS itself. The work of Chapter 6 gave AEFs for rhodamine probes that were a little higher, at $\sim 1 \times 10^{6}$, indicating that, despite some batch-to-batch variability, the 
AEF may be characteristic of the average enhancement of these substrates for a variety of different analytes.

The single-molecule enhancement factor (SMEF), which becomes available through the use of BiASERS, gave us a measure of the EFs required to see single molecules. It turns out that values of $10^{8}$ were sufficient to see SM signals of resonant dyes such as rhodamine and nile blue. This is up to six orders of magnitude smaller than some reports have suggested, and represents a significant rescaling in the perception of what is required for SM-SERS. Put another way, these results showed that SM detection could be observed in $0.05 \mathrm{sec}$ for probes with differential SERS cross-sections of $10^{-20} \mathrm{~cm}^{2} / \mathrm{sr}$, which is four orders of magnitude smaller than what was usually assumed in the literature $[40,62,141]$. The extension of these results is that, assuming ideal conditions and no photobleaching, at longer integration times SMEFs of $10^{5}$ may be sufficient for the SM detection of dyes such as rhodamine and $\mathrm{CV}$ at resonance. This is a staggering nine orders of magnitude smaller than what was normally assumed for many years in the literature, although it should be noted that these ideal conditions may not be achievable for many probes.

A natural extension of the work on SMEFs was the recognition that it should be possible to achieve SM detection of non-resonant probes. In many ways, research in SERS has "loaded the dice" in favour of detecting molecules with large crosssections, which benefit from resonance with the incident excitation. In terms of applications, however, it is obvious that a great proportion of the analytes of interest, from biological molecules, to common chemicals in the environment, are non-resonant. Thus, an important question in this sense is: "What is the minimum Raman cross-section that can be measured as a single molecule?" After careful experiments, we were able to show that SM detection is possible for non-resonant dyes with bare Raman cross-sections down to $10^{-30} \mathrm{~cm}^{2} / \mathrm{sr}$ under typical Raman conditions. Such a low cross-section, measured in our case for the nucleotide adenine, is representative of the majority of the probes of applied interest, such as biological molecules or those of forensic or environmental interest. Thus this thesis offers positive news in terms of applications. Further, in our opinion, this work provides the first rigorous proof of SM detection of non-resonant probes, given 
that re-analysis of previous examples of supposed SM detection has revealed some controversial issues [41, 42].

Although these experiments necessitated careful optimization of the SERS substrate and colloidal system to begin with, they nonetheless demonstrate a proof of principle that has previously not been demonstrated satisfactorily in the field; that is, non-resonant probes, which represent the majority of probes of practical interest can be measured at the SM level.

Another important ingredient in this process was the use of isotopically edited analogues as ideal partners in BiASERS experiments. When comparing SERS substrates or conditions, the use of isotopic analogues in combination with the EF protocol provides a valuable tool for a SERS practitioner. In many ways, such isotopic probes can be considered a metrology standard for a variety of potential experiments in SERS, with the expectation that a researcher could purchase such probes and use them to quantify the lowest detection limit of their system.

Taken together, the results of this thesis show a remarkable consistency in that the observed SMEFs point towards an upper maximum in the region of $\sim 10^{11}$ as a best-case scenario, which is perfectly in line with maximum EFs predicted theoretically $[2,28,29,43]$. This maximum was obtained in our work for separate experiments on both non-resonant and resonant probes, and for substrates prepared from Lee \& Meisel colloids and borohydride-reduced colloids. As aggregated colloids are known to give amongst the greatest enhancements of any SERS substrate [21], this value points towards a best-case scenario in terms of SMEFs in SERS. Further, such a maximum value is supported by results obtained by temperature dependent vibrational pumping (TDVP) experiments, which offers a completely different method for obtaining the SERS cross-section and SMEF. Other experimental approaches in the literature are also consistent with such EFs $[72,138]$.

Importantly, the placement of maximum experimental SMEFs within the expected limits of electromagnetic theory means that SM detection is possible for a wide variety of potential analytes without the need for any extra enhancement from chemical effects. While chemical effects and enhancements are of considerable 
interest and may be particularly relevant for certain analytes, we believe that for too long some of the literature has assumed their existence on a prima facie basis as a result of the erroneously large previous estimates of the SMEF.

In terms of general impact, the work of this thesis provides an interested SERS researcher with an effective "toolbox" of methods for estimating EFs in several common SERS situations, and highlights suitable reference standards for this endeavour such as the liquid 2B2MP; similarly, $\mathrm{N}_{2}$ can be used as a reference to calibrate SM events for air objectives. Not all the techniques described within this thesis are new, but their use in combination, along with suitable definitions and careful experiments, represents a new and powerful approach to illuminating some of the most fundamental problems in SERS. It is expected that many people in the field will be interested in these methods and results, as they enable one to begin to put accurate values to the SERS effect and the enhancements available for typical SERS substrates and conditions. In terms of applications, we have obtained an experimental value for the lowest bare Raman cross-section measurable as a single molecule under typical SERS conditions. The new information provided in this thesis would be particularly encouraging to those looking at SMSERS of non-resonant probes, in particular for biological applications.

Finally, the results represent a fulfilment of the goals of this thesis in terms of the EF problem. Since the first reports of SM detection, EFs of $10^{14}$ have continued to proliferate amongst the literature, becoming a standard feature of introductions and reviews on the topic. With the benefit of a more structured and rigorous approach, we have been able to provide more reliable values for maximum EFs in SERS. 


\section{Appendix A}

\section{MEASURING THE SCATTERING VOLUME}

\section{A.1 Theoretical Considerations}

\section{A.1.1 The need to characterize the scattering volume}

A proper characterization of the scattering volume of specific objectives is a critical part in the quantification of the SMEF (explored in Chapter 5). This is necessary because when measuring the SMEF we are no longer comparing like-withlike signals in terms of the Raman and SERS experimental situations.

When measuring the AEF, we simply compare Raman signals of two solutions under exactly the same conditions. The cross-section of one can then be deduced from that of the other provided the concentrations of the samples are known. In contrast, measurement of the SMEF is not as straightforward. To obtain the SERS cross-section for a SM event for calculation of the SMEF, we must compare the SERS signal, $I_{\mathrm{SERS}}^{\mathrm{SM}}$, to the average Raman signal of a single reference molecule, $\left\langle I_{\operatorname{Ref}^{\prime}}^{\mathrm{SM}}\right\rangle$ at the centre of the focal plane under the same experimental conditions.

However, experimentally we only have access to the total Raman signal $I_{\text {Ref }}^{\text {Tot }}$ aris- 
ing from many reference molecules at concentration $c$. It is therefore necessary to precisely characterize our scattering volume (excitation and collection) to obtain the desired quantity $\left\langle I_{\text {Ref }}^{\mathrm{SM}}\right\rangle$.

The following gives an overview of the background and experimental methods for determining the scattering volume of a given objective. This work is not part of the thesis, as such, but is necessary to understand the calculation of the EFs. Readers interested in further details are directed to Refs. [56, 142].

\section{A.1.2 General Principles}

The scattering volume is effectively characterized by the excitation and detection profiles of the system, which are naturally intertwined but can be deconvoluted by performing appropriate experiments (e.g. changing the confocal pinhole or slit sizes).

The excitation intensity profile, $I(\rho, z)\left[\mathrm{W} \mathrm{m}^{-2}\right]$ (axial symmetry is assumed here) describes the laser intensity as a function of position, which is inevitably nonuniform. The detection efficiency profile $\eta(\rho, z)$ is strongly dependent on the particular experimental setup. In our case, the collected light enters a square confocal pinhole, which also serves as an entrance slit for the monochromator. In this setup, only light emitted from a point source at the centre of the focal plane is detected with maximum efficiency $\eta_{0}$.

Assuming $I(\rho, z)$ and $\eta(\rho, z)$ are known we can define an effective scattering volume $V_{\text {eff }}\left[\mathrm{m}^{3}\right]$ as:

$$
V_{\text {eff }}=\int_{\rho=0}^{\rho=\infty} 2 \pi \rho \int_{z=-\infty}^{z=+\infty} \frac{I(\rho, z)}{I_{0}} \frac{\eta(\rho, z)}{\eta_{0}} d \rho d z .
$$

$V_{\text {eff }}$ corresponds to the volume from which the same Raman signal would be observed if the excitation and detection efficiency were both uniform and equal to their maximum values, $I_{0}$ and $\eta_{0}$. Note that $V_{\text {eff }}$ is smaller than the actual volume probed by our system. This approach is an extension to that described in Ref. [61], where an effective height was defined to characterize the detection efficiency (non-uniform excitation was then ignored). 
We now show how we characterize and measure the effective scattering volume. Our approach here is to present the simplest approximations, which still capture most of the important aspects of the problem. More elaborate treatments can be found in the literature $[2,56,142]$. We then give a description of how these ideas of confocal microscopy apply to the problem of obtaining the SMEF.

\section{A.2 Excitation Profile}

To determine the excitation, we first approximate the laser as having a Gaussian beam profile with axis along $z$. Following standard definitions [143, 144], the intensity profile is therefore:

$$
I(\rho, z)=I_{0} \frac{w_{0}^{2}}{w(z)^{2}} \exp \left(-\frac{2 \rho^{2}}{w(z)^{2}}\right)
$$

where $I_{0}\left[\mathrm{~W} \mathrm{~m}^{-2}\right]$ is the intensity at the centre of the focal plane, $w_{0}[\mathrm{~m}]$ is the waist of the Gaussian beam, and

$$
w(z)=w_{0} \sqrt{1+\frac{z^{2}}{z_{0}^{2}}} \quad, \text { where } z_{0}=\frac{\pi w_{0}^{2}}{\lambda} .
$$

The power $P_{0}[\mathrm{~W}]$ of such a Gaussian beam is:

$$
P_{0}=\frac{\pi w_{0}^{2}}{2} I_{0}
$$

Since $P_{0}$ is easily measured (with a power meter), the only unknown parameter is the waist $w_{0}$.

\section{A.3 Detection Efficiency Profile}

The detection efficiency profile is largely determined by the shape and size of the entrance slits of the monochromator. An image of the spot area is formed on this aperture, and in a first approximation, the part of the image that is inside the aperture is detected while the rest is simply cut out. This ignores diffraction effects, 
which is almost always correct since most spectrometers magnify the size of the real spot on the entrance slit. The dimensions of the image on the slit is related to the actual dimensions at the sample by a factor $X$, which is the magnification factor of the microscope objective times any other magnifications introduced by the collecting optics (in our case it gives a factor of $0.56 \times$ the magnification of the objective).

For our setup, in which the slits form a square aperture ${ }^{1}$ of dimension $2 L$ [m] (edge length), the detection efficiency is:

$$
\begin{array}{r}
\eta(x, y, z)=\eta_{\perp}(z) \quad \text { if }-L / X \leq x, y \leq L / X \\
\eta(x, y, z)=0 \quad \text { otherwise. }
\end{array}
$$

The axial detection efficiency $\eta_{\perp}(z)$ also depends strongly on the optical setup, in particular the wavelength, microscope objective, and confocal pinhole size. It is a maximum at $z=0$ and decreases away from the focal plane. In our confocal setup, this decrease is over a very small distance, characterized by the confocal depth.

Within these assumptions it is possible to further simplify the integrals in Eqn. A.1 when the entrance slit is sufficiently open. More precisely, if $L \gg w_{0} X$, then only a negligible part of the image of the Gaussian beam excitation is cut out by the slits, and the effect of the lateral detection efficiency can be ignored. In this approximation, the signals are independent of $L$. This condition is easy to check experimentally by gradually closing the slits (to the point where this approximation is no longer valid).

In this case, the $\rho$ dependence of $\eta$ can be ignored, and the integral over $\rho$ in Eqn. A. 1 becomes independent of $z$ and is simply equal to $P_{0}$, the incident power. This leads us to introduce an effective height, $H_{\text {eff }}[\mathrm{m}]$, defined as:

$$
H_{\mathrm{eff}}=\int_{z=-\infty}^{z=+\infty} \frac{\eta_{\perp}(z)}{\eta_{0}} d z .
$$

\footnotetext{
${ }^{1}$ In our system, a Jobin-Yvon LabRam, the pinhole size $d$ actually corresponds to the length of the diagonal of the square aperture and is accordingly related to $L$ by $d=2 \sqrt{2} L$.
} 
We can then express the total signal obtained from the Raman reference $I_{\operatorname{Ref}}^{\mathrm{Tot}}$ as:

$$
I_{\text {Ref }}^{\text {Tot }}=c \sigma_{d} P_{0} H_{\text {eff }}
$$

and the effective scattering volume $V_{\text {eff }}$ as:

$$
V_{\text {eff }}=A_{\text {eff }} H_{\text {eff }}, \quad \text { where } \quad A_{\text {eff }}=P_{0} / I_{0}=\frac{\pi w_{0}^{2}}{2},
$$

is the effective surface area $\left[\mathrm{m}^{2}\right]$ of the Gaussian excitation. This generalizes the concept of effective height of the scattering volume, introduced in Ref. [61], to the common case of non-uniform (Gaussian) excitation. It is important to note however that this approach is only valid when the entrance slits are sufficiently open, which allows one to "factorize" the respective contributions of excitation and detection. As discussed later, it is no longer valid for smaller slits and will therefore fail in a truly confocal regime. $H_{\text {eff }}$ cannot be simply viewed as the confocal depth of the microscope [56].

Having defined more accurately the excitation profile and detection profile, we now describe the experimental methods to measure their respective characteristics, and in particular $w_{0}$ (and therefore $A_{\text {eff }}$ ) for excitation, and $\eta_{\perp}(z)$ (and therefore $H_{\text {eff }}$ ) for detection.

\section{A.4 Beam Profiling Experiments}

\section{A.4.1 Scan over an edge}

One simple approach to determine the waist of the excitation profile is to measure the Raman signal of a strongly absorbing thin film (typically silicon) as the objective is focused on the film and scanned laterally over a sharp (cleaved) edge (scanning knife-edge method [145]). Because of strong absorption, all the Raman signal comes from the Si wafer surface (and therefore from the focal plane $z=0$ ). The first step is to ensure that the slits are sufficiently open to collect all the signal from the laser spot, which can be checked by monitoring the Raman signal while 


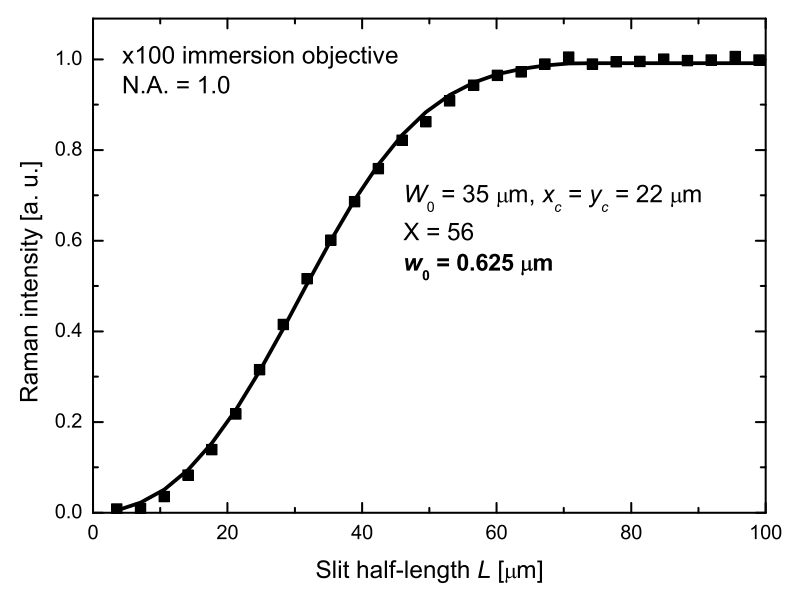

Figure A.1: Raman signal from a Si substrate at $633 \mathrm{~nm}$ as a function of entrance slits sizes. A $\times 100$ immersion (water) microscope objective with N.A. of 1.0 was used here and an additional scaling factor of 0.56 exists for our particular optical setup, resulting in a magnification of $X=56$ from sample to its image at the square aperture of the entrance slits. Symbols correspond to experimental values at laser focus on the Si substrate and the solid line is a fit to the data using Eqn. A.10.

closing the slits. We are then in a situation where the detection efficiency is uniform (no lateral restriction by the slits, and no $z$ dependence since all the signal comes from the focal plane).

Assuming we scan the spot along the $x$ axis and that the edge is at $x=x_{0}$ (silicon wafer on $x<x_{0}$ ), the Raman intensity dependence is then:

$$
I(x)=\frac{I_{\max }}{2}\left[1+\operatorname{erf}\left(\frac{\sqrt{2}\left(x_{0}-x\right)}{w_{0}}\right)\right],
$$

where $\operatorname{erf}(x)$ is the error function. This intensity profile is quite sensitive to the value of $w_{0}$, and this parameter can therefore be extracted from a fit to the experimental data. An example is shown in Fig. A.2. Note that there is a slight overshoot of the signal as we reach the Si edge; this is purely an "edge" effect to do with the additional contribution from the diffracted beam on the side of the wafer when the beam is half-way through the edge and can be ignored when fitting to the above expression. For a $\times 10$ objective as in our example, a waist of $w_{0}=4.6 \mu \mathrm{m}$ is derived. The same experiment can also be done out of focus $(h=150 \mu \mathrm{m}$ beyond 


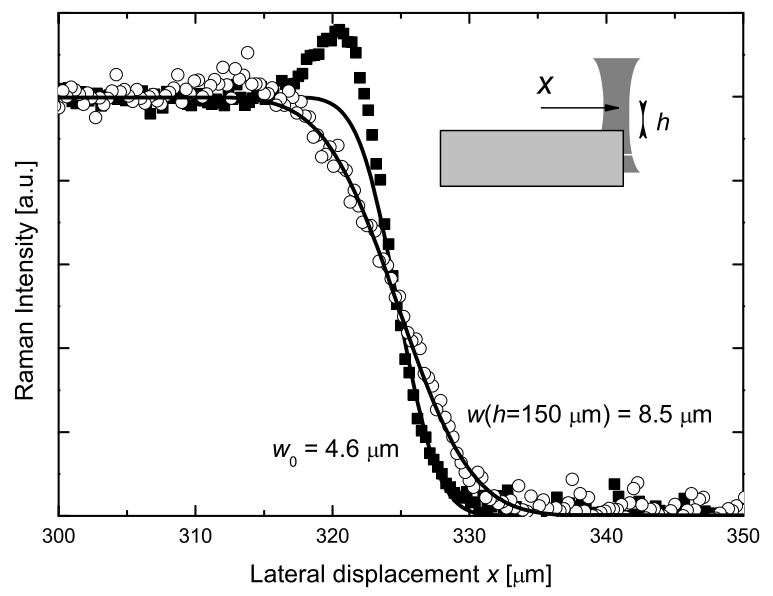

Figure A.2: Raman signal from silicon at $633 \mathrm{~nm}$ as a function of displacement while scanning over a Si substrate edge. A $\times 10$ microscope objective with N.A. of 0.25 was used here. Symbols correspond to experimental values at laser focus on the Si substrate (solid squares) and at a distance $f=150 \mu \mathrm{m}$ beyond focus (open circles). Solid lines are fits to the data. The overshoot observed when reaching the Si edge is not taken into account for the fits.

focus in the example of Fig. A.2). The derived waist is then $w(h) \approx 8.5 \mu \mathrm{m}$, which for a Gaussian beam can also be predicted from Eqn. A.3 to be $w(h)=8 \mu \mathrm{m}$, in good agreement with the measured value.

It is important to note that the accuracy of this technique decreases substantially for narrowly focused beams, typically for $w_{0}<2 \mu \mathrm{m}$. It is in fact impractical for high-magnification, high-N.A. objectives with near-diffraction-limited spot sizes.

\section{A.4.2 Slit size dependence}

One method to determine the waist, $\omega_{0}$, of the excitation profile is to use the monochromator's slits (which in our system is also the confocal pinhole) as a spatial filter. When closing the slits in a controlled way, an increasing part of the spot image is cut out from detection, and the signal decreases correspondingly. The laser is set at focus, $z=0$, on a Si substrate to avoid any influence of the $z$-dependence. 
We take our setup, in which the slits form a square aperture of dimension $2 L$. An image of the Gaussian excitation, with waist $W_{0}=w_{0} X$, is formed in this aperture and centred on $x=0, y=0$. (As explained in Section A.3, the waist of the image $W_{0}$ is related to the actual waist of the beam by the magnification factor $X$.) We will allow for a possible (almost always present) slight misalignment, which means that the aperture may be slightly off-centre, at $x=x_{c}, y=y_{c}$ compared to the beam image. The dependence of the Raman intensity with slit size (characterized by $L$ ) can then be predicted by integrating the intensity profile of the beam image within the aperture, as given by [56]:

$$
\begin{aligned}
& \frac{I(L)}{I_{\max }}=\frac{1}{4}\left[\operatorname{erf}\left(\frac{\sqrt{2}}{W_{0}}\left(x_{c}+L\right)\right)-\operatorname{erf}\left(\frac{\sqrt{2}}{W_{0}}\left(x_{c}-L\right)\right)\right] \\
& \times\left[\operatorname{erf}\left(\frac{\sqrt{2}}{W_{0}}\left(y_{c}+L\right)\right)-\operatorname{erf}\left(\frac{\sqrt{2}}{W_{0}}\left(y_{c}-L\right)\right)\right] .
\end{aligned}
$$

This expression simplifies for a perfect alignment to:

$$
\frac{I(L)}{I_{\max }}=\left[\operatorname{erf}\left(\frac{\sqrt{2} L}{W_{0}}\right)\right]^{2},
$$

where erf is the error function. We then fit the above expressions to experimental data to derive $W_{0}$. Figure A.1 shows an example of the intensity profile as a function of entrance slit sizes for the $\times 100$, N.A. $=1.0$ water immersion objective. From the above considerations, a waist of $w_{0} \approx 0.625 \mu \mathrm{m}$ is deduced along with the existence of a slight misalignment $\left(x_{c}=y_{c}=22 \mu \mathrm{m}\right)$.

\section{A.4.3 Axial detection efficiency}

Once $w_{0}$ is determined, the excitation profile is fully characterized and we can now focus on the axial detection efficiency $\eta_{\perp}(z)$. A standard approach here is to measure the Si Raman signal $I(f)$ as a function of distance $f$ from the focal plane on the substrate [61]. A bell-shaped profile is obtained, whose FWHM is defined as the confocal depth. However, concluding that this profile corresponds to the axial detection efficiency $\eta_{\perp}(z)$ can be erroneous for at least one reason: the size of the illuminated area on the Si substrate increases when going away from or 


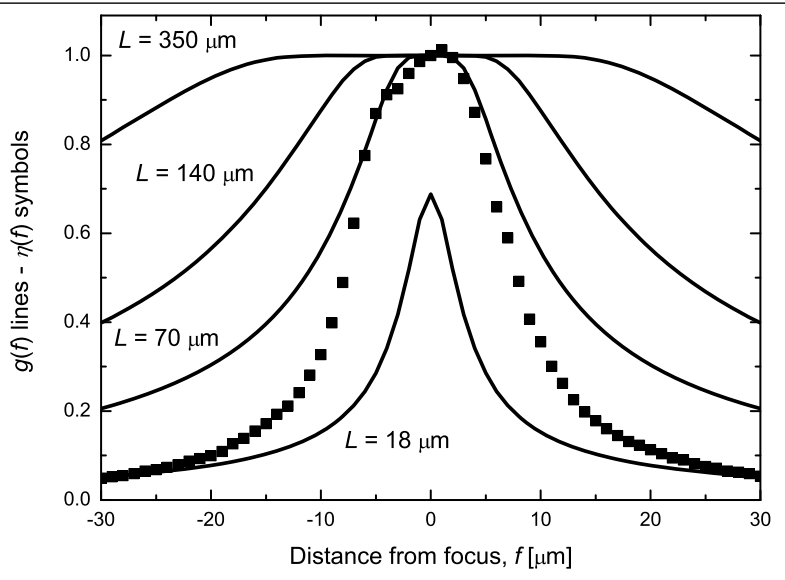

Figure A.3: Raman intensity from a Si substrate at $633 \mathrm{~nm}$ as a function of distance $f$ from the focal plane (symbols). A $\times 100$ immersion (water) microscope objective with N.A. of 1.0 was used here and the slits were fully opened $(L=354 \mu \mathrm{m})$. The solid lines show the function $g(f)$ obtained from Eqn. A.13 for four representative slit sizes. For the largest slit size, $g(f)$ remains nearly constant in the range of interest, and the symbols are therefore a faithful representation of the axial detection efficiency profile $\eta_{\perp}(f)$.

beyond focus (due to the Gaussian beam profile). The periphery of this area may then be cut out from detection because of the lateral detection efficiency (which depends on the entrance slit size).

Because of this, a bell-shaped curve can be obtained from $I(f)$ even if $\eta_{\perp}(z)$ was constant. It is therefore necessary to deconvolve this effect from the actual contribution of $\eta_{\perp}(z)$, or at least ensure that it does not play a role. If we neglect the finite penetration depth into $\mathrm{Si}$, all the Raman signal comes from the Si surface at $z=f$ and we then have for a given slit size $L, I(f, L)=I_{\max } \eta_{\perp}(f) g(f, L)$ with

$$
g(f, L)=\int_{x=-L}^{x=L} \int_{y=-L}^{y=L} d x d y I(x, y, f) / I_{\max }
$$

$g(f, L)$ contains contributions from both the non-uniform excitation and the lateral detection efficiency (governed by the slit size $L$ ). It can moreover be calculated from Eqn. A.10, or in the case of ideal alignment from Eqn. A.11:

$$
g(f, L)=\left[\operatorname{erf}\left(\frac{\sqrt{2} L}{X w(f)}\right)\right]^{2}
$$


where $w(f)$ is given in Eqn. A.3. The function $g(f, L)$ is precisely the origin of the problem discussed above. If this function is not constant, then $I(f)$ does not directly reflect the axial detection efficiency, but a convolution of it with a complex function of excitation profile and lateral detection efficiency.

We illustrate this on a specific example, where $a \times 100$ N.A. $=1.0$ water immersion objective is used at $633 \mathrm{~nm}$. The magnification factor between spot and image is then $X=56$. The waist of the Gaussian beam excitation measured by the slit dependence experiment was: $w_{0}=0.625 \mu \mathrm{m}$, corresponding to a waist of $W_{0}=X w_{0}=35 \mu \mathrm{m}$ in the image. Examples of the profile of $g(f)$ for these parameters, calculated from Eqn. A.13, are given in Fig. A.3 for different slit sizes $L$. It is clear from these that for the smallest slit sizes, a bell-shaped curve is already obtained from $g(f)$ and is not a result of the axial detection profile. This effect must be taken into account for an accurate determination of the confocal profile. For the largest slit size, however, $g(f)$ remains almost constant over a wide range of distances.

As such, it is critical in measurement of the axial dependence to have the slits sufficiently open so as to ensure than none of the signals emitted from the scattering volume are cut out, the reasons for which are expanded in detail in the supplementary material of Ref. [56]. As long as the slits are fully open, the lateral detection efficiency does not influence the signals. For smaller slit sizes, $I(f)$ is not a truthful representation of $\eta_{\perp}(f)$.

What we need for our purpose here is the best possible characterization of the scattering volume. From the previous arguments, this is more easily achieved when the slits are fully opened since $g(f) \approx 1$ and has only a negligible effect. In this case, $I(f) \propto \eta_{\perp}(f)$ is a faithful representation of the axial detection efficiency and the value of $H_{\text {eff }}$ can be taken from the FWHM of the bell-shaped curve resulting from the axial dependence. We then have the two parameters we need, $A_{\text {eff }}$ and $H_{\text {eff }}$ to calculate the scattering volume by Eqn. A.8. The characteristic values obtained for the beam profiling experiments of objectives used in this thesis are given in Table 3.1 . 


\section{A.5 Obtaining the SMEF}

\section{A.5.1 Calculating the SMEF from SM-SERS event}

Now that we have characterized the beam profile in terms of $A_{\text {eff }}$ and $H_{\text {eff }}$, we can estimate the effective scattering volume by Eqn. A.8. This is then used to determine the number of molecules in the scattering volume for a reference compound (liquid or gas) with a known cross-section, which is measured under Raman conditions immediately after the SM experiments. The intensity of the reference compound per molecule along with the known cross-section for that molecule then gives us a calibration factor to get the effective SM-SERS cross-section. In order to determine the SMEF (or apparent SERS cross-section) for a given SM-SERS event, one needs to compare the SM-SERS signal $I_{\text {SERS }}^{\text {SM }}$ to the average Raman signal of a single reference molecule $\left\langle I_{\text {Ref }}^{\mathrm{SM}}\right\rangle$ under exactly the same conditions. By choosing only the strongest SM-SERS events, we ensure that they correspond to a molecule at the centre of the focal plane, where the excitation density $I_{0}$ and collection efficiency $\eta_{0}$ are maximum. Only the total signal $I_{\mathrm{Ref}}^{\mathrm{Tot}}$ of the bare Raman intensity of a large concentration of reference molecules is measurable in practise. This must be measured under experimental conditions where the scattering volume can be accurately characterized, i.e. with slits fully open. The average Raman signal of a single reference molecule, $\left\langle\mathrm{I}_{\mathrm{Ref}}^{\mathrm{SM}}\right\rangle$, can then be obtained as:

$$
\left\langle I_{\mathrm{SM}}^{\mathrm{Ref}}\right\rangle=\frac{I_{\mathrm{Ref}}^{\mathrm{Tot}}}{c N_{a} V_{\mathrm{eff}}},
$$

where $N_{a}=6.02 \times 10^{23}$ is Avogadro's number, and $c$ is the concentration of the reference. We can then obtain the estimated SM-SERS cross-section, $\frac{d \sigma_{\text {SERS }}^{\mathrm{SM}}}{d \Omega}$, from the fitted intensity of the SM event, $I_{\text {SERS }}^{\mathrm{SM}}$, by:

$$
\frac{d \sigma_{\mathrm{SERS}}^{\mathrm{SM}}}{d \Omega}=\frac{I_{\mathrm{SERS}}^{\mathrm{SM}}}{\left\langle I_{\mathrm{Ref}}^{\mathrm{SM}}\right\rangle} .
$$

Values for the concentrations and cross-sections for the 2-bromo-2-methylpropane (2B2MP) (liquid reference) and $N_{2}$ (gas reference) are given in Table A.1. 
Table A.1: Table of the properties of the references used for calibration of the SM signals obtained from BiASERS measurements. $c$ is the concentration and $\bar{v}_{i}$ and $\frac{d \sigma_{\mathrm{RS}}}{d \Omega}$ are the frequency of the reference mode used for calibration and its absolute differential Raman cross-section, respectively. The reported cross-sections were obtained from Ref. [6]. See Chapter 5 for further details.

\begin{tabular}{lccc}
\hline \hline Reference & $\begin{array}{c}c \\
{[\mathrm{M}]}\end{array}$ & $\begin{array}{c}\bar{v}_{i} \\
{\left[\mathrm{~cm}^{-1}\right]}\end{array}$ & $\begin{array}{c}\frac{d \sigma_{\mathrm{RS}}}{d \Omega} \\
{\left[\mathrm{cm}^{2} / \mathrm{sr}\right]}\end{array}$ \\
\hline 2B2MP & 8.76 & 516 & $5.4 \times 10^{-30}$ (liq) \\
$\mathrm{N}_{2}$ & 0.0319 & 2331 & $2 \times 10^{-31}$ (gas) \\
\hline \hline
\end{tabular}

As an example, for the BiASERS experiments presented in Chapter 5, the $516 \mathrm{~cm}^{-1}$ mode of $2 \mathrm{~B} 2 \mathrm{MP}$ had a total Raman intensity of $I_{\mathrm{Ref}}^{\mathrm{Tot}}=1.7 \times 10^{4} \mathrm{cts} / \mathrm{s}$. The concentration of pure $2 \mathrm{~B} 2 \mathrm{MP}$ is $c=8.76 \mathrm{M}$, so according to Eqn. A.14, a single molecule of $2 \mathrm{~B} 2 \mathrm{MP}$ at the centre of the beam has a reference average Raman intensity of $\left\langle I_{\mathrm{SM}}^{\mathrm{Ref}}\right\rangle=2.5 \times 10^{-7} \mathrm{cts} / \mathrm{s}$. This estimate can then be compared to the SM-SERS signals of a single dye molecule in the BiASERS method to derive the SERS differential cross-section and the corresponding SMEF.

It is important to note that even if these things are measured as thoroughly as possible, some uncertainty will still exist in the exact SMEF, possibly by a factor of $\sim 2$. Such accuracy is sufficient, however, for comparison of most SERS substrates or SERS probes, and it represents an unprecedented degree of accuracy compared to the many-orders-of-magnitude spread found in the different claims in the literature.

\section{A.5.2 Calculating the SMEF from TDVP}

Temperature-dependent vibrational pumping (TDVP) measurements provide us with pumping cross-sections, which cannot be readily compared to the non-SERS cross-sections to obtain an SMEF for two reasons. Firstly, they correspond to a statistical average, heavily biased towards the largest cross-sections [69] exhibited 
by the SERS substrate. For a collection of SERS hot-spots, this was shown [43] to be approximately half the maximum cross-section. Secondly, the pumping crosssection is a total SERS cross-section. In order to deduce the differential SERS cross-section, we must first assume that the non-radiative contribution is negligible compared to the radiative part. This approximation is in fact implicit in the use of the corrected lifetime method outlined in the background material (Section 2.5). We believe it is a reasonable approximation for a hot-spot (which must have a large radiative enhancement).

Moreover, by integrating the differential SERS cross-section at a hot-spot over all possible emission directions, one can show there is a factor $8 \pi / 3$ between differential and integrated radiative SERS cross-section at the hot-spot. Also, because the pumping cross-section is an average of molecules at hot-spots with different EFs, we may apply a correction to obtain the maximum cross-section depending on the colloid system and EF distribution. For a Pareto distribution, an effective model for such colloid systems, it was found that the average pumping enhancement was a factor $\sim 2$ smaller than the maximum enhancement [43]. With these corrections, the maximum differential SERS cross-section $d \sigma_{\text {SERS }} / d \Omega$ on the substrate can be deduced from the pumping cross-section $\sigma_{\text {pump }}$ using the relation:

$$
\frac{d \sigma_{\text {SERS }}}{d \Omega} \approx \frac{3}{4 \pi} \sigma_{\text {pump }}
$$

From there the maximum SMEF can be deduced as before, taking into account the non-SERS cross-sections derived before.

\section{A.6 Spectral Analysis}

As with all spectroscopies, curve fitting and determining the area under peaks forms a considerable part of the analysis in Raman spectroscopy and SERS. We describe briefly here the main methods used in this thesis. 


\section{A.6.1 Least-squares fitting to pseudo-Voigt function}

A pseudo-Voigt function is a mixture of a Gaussian and a Lorentzian function [146] and can be expressed as:

$$
I(x)=\alpha I_{\max }\left[\exp -4 \ln 2 \frac{\left(x-x_{0}\right)^{2}}{\Gamma^{2}}\right]+\frac{(1-\alpha) I_{\max }}{1+\frac{4\left(x-x_{0}\right)^{2}}{\Gamma^{2}}}
$$

where $\alpha$ is the relative proportion of Gaussian to Lorentzian in the function (i.e. $\alpha=0$ is a Lorentzian), $I_{\max }$ is the maximum peak intensity at $x=x_{0}$, and $\Gamma$ is the peak width. Spectral peaks are fit to this function by solving a linear least-squares fit. Note that this form of the equation normalizes the Gaussian and Lorentzian to a single peak width, $\Gamma$, for fitting purposes. Routines in Excel (for a singlet or doublet peak) are then used to apply the fittings to a time-series of many spectra with the user having the ability to fix parameters such as $\alpha$ when appropriate.

\section{A.6.2 Linear least-squares deconvolution}

To select valid events from a large number of BiASERS spectra, which may also include erroneous spectra arising from photodegradation products or contaminants, linear least-squares deconvolution is a particularly useful technique (used mainly in Chapter 7). For this technique, two linear spectral components are obtained from experimental SERS spectra of the two probe molecules measured individually. These reference spectra are then used in the linear deconvolution along with a linear background to obtain fits of the relative proportions of each individual molecule in each BiASERS event (for a time series of many events).

$$
I_{\mathrm{BiASERS}}\left(\bar{v}_{i}\right)=\alpha I_{\mathrm{BPE}}\left(\bar{v}_{i}\right)+\beta I_{\mathrm{BTZ}}\left(\bar{v}_{i}\right)+\gamma \bar{v}_{i}+\delta,
$$

where $I_{\text {BiASERS }}\left(\bar{v}_{i}\right)$ is the bi-analyte SERS (BiASERS) spectrum to be fitted and $I_{\mathrm{BPE}}\left(\bar{v}_{i}\right)$ and $I_{\mathrm{BTZ}}\left(\bar{v}_{i}\right)$ are the average SERS reference spectra of the two molecules obtained experimentally by separate measurement under identical conditions. The fit also includes a linear background characterized by slope $\gamma$ and intercept $\delta$. $\alpha, \beta$, 
$\gamma$, and $\delta$ are the only fitting parameters and are uniquely (and easily) determined by the linear least-squares fitting routine.

The inclusion of a linear background would be sufficient to deal with any fluctuating SERS background (broad background feature) as long as the spectral window for fitting was small enough (i.e. $\sim 200-300 \mathrm{~cm}^{-1}$ ). Indeed, in the case of nonresonant probes, a small spectral window is usually desirable, because the strong likelihood of spurious peaks in various parts of the spectra would hinder analysis over the entire spectral window.

The validity of the fit (or "goodness-of-fit" value) was determined by a $\chi^{2}$-parameter:

$$
\chi^{2}=1-\frac{\sum\left(y_{i}-f_{i}\right)^{2}}{\sum y_{i}^{2}}
$$

which was normalized to the spectral intensity of the event, where $y_{i}$ and $f_{i}$ are the spectral intensities of the actual data and fit, respectively. Fits below a certain intensity (below noise level) and those with a "goodness-of-fit" below an appropriately chosen threshold were discarded along with any remaining non-physical fits. In practise, the linear deconvolution was performed using a customized Matlab script.

\section{A.6.3 Modified principal component analysis (MPCA)}

Principal component analysis (PCA) is a mathematical procedure that effectively reduces a set of data to a number of "principal components" that are most representative of the data. The technique is used in a large number of applications in data processing and file compression, where it is effective in compressing images while retaining the main features of the image. In our case, the technique is another in a suite of methods for analysis of large numbers of spectra, as obtained from time series or map measurements. We present here a basic overview of the approach and refer the reader to other sources for further details [52, 147].

PCA is mathematically defined [147] as an orthogonal linear transformation that 
transforms a set of data to a new coordinate system such that the greatest variance by any projection of the data comes to lie on the first coordinate (called the first principal component), the second greatest variance on the second coordinate, and so on. PCA is theoretically the optimum transform for the given data in leastsquares terms. In statistical terms, the technique performs multivariate analysis by eigenvalue decomposition of a data covariance matrix.

In this work, we use a modification of the PCA method (MPCA), developed exactly for the purpose of resolving large numbers of BiASERS Raman spectra into two main components (those of the individual analytes) [52]. Briefly, a time series (or map) of spectral intensities is formulated as a $T \times N$ matrix, $M$, where $\mathrm{T}$ is the number of spectra and $\mathrm{N}$ are the wavelengths of the spectral region of interest.

$$
M=\overbrace{\left(\begin{array}{ccccc}
I_{t_{1}}^{\lambda_{1}} & I_{t_{1}}^{\lambda_{2}} & \ldots & \ldots & I_{t_{1}}^{\lambda_{N}} \\
I_{t_{2}}^{\lambda_{1}} & I_{t_{2}}^{\lambda_{2}} & \ldots & \ldots & I_{t_{2}}^{\lambda_{N}} \\
\ldots & \ldots & \ldots & \ldots & \ldots \\
I_{t_{T}}^{\lambda_{1}} & I_{t_{T}}^{\lambda_{2}} & \ldots & \ldots & I_{t_{T}}^{\lambda_{N}}
\end{array}\right)}^{N \text {-wavelengths }}\} \mathrm{T} \text { - times. }
$$

We then subtract the mean of each spectra to obtain a spectrum of zero mean intensity. Then, the covariance matrix $V(N \times N)$ for the $N$ column vectors of the matrix $\hat{M}(T \times N)$ is calculated:

$$
V=\left(\operatorname{cov}\left(\hat{I}_{t}^{\lambda_{j}}, \hat{I}_{t}^{\lambda_{k}}\right)\right)
$$

where $\operatorname{cov}\left(\hat{I}_{t}^{\lambda_{j}}, \hat{I}_{t}^{\lambda_{k}}\right)=\operatorname{cov}\left(\hat{I}_{t}^{\lambda_{k}}, \hat{I}_{t}^{\lambda_{j}}\right)$ is the covariance of the intensity columns at $\lambda_{j}$ and $\lambda_{k}$, calculated here using the unbiased estimator for the covariance:

$$
\operatorname{cov}\left(\hat{I}_{t}^{\lambda_{j}}, \hat{I}_{t}^{\lambda_{k}}\right)=\sum_{i=1}^{T} \frac{\left(\hat{I}_{t_{i}}^{\lambda_{j}}-\left\langle\hat{I}^{\lambda_{j}}\right\rangle\right)\left(\hat{I}_{t_{i}}^{\lambda_{k}}-\left\langle\hat{I}^{\lambda_{k}}\right\rangle\right)}{(T-1)}
$$

with $\langle\ldots\rangle$ denoting the time-average:

$$
\left\langle\hat{I}^{\lambda_{j}}\right\rangle=\frac{1}{T} \sum_{i=1}^{T} \hat{I}_{t_{i}}^{\lambda_{j}}
$$


The next step is where the "dimensionality reduction" concept comes into play. We first obtain the $N$ eigenvalues and $N$ corresponding eigenvectors of the covariance matrix $V$. The eigenvalues are all real and positive since this matrix is real and symmetric, and can therefore be ordered from largest to smallest. The corresponding eigenvectors are the principal components in order of significance (greatest variance). The first eigenvector $f_{1}^{\lambda_{j}}(j=1 . . N)$, for example, can be considered to be a function of wavelength that captures the most important feature in the overall set of data. Similarly, the second eigenvector $f_{2}^{\lambda_{j}}$ captures the second most important feature in the data, and so on.

The last step is to obtain the table of coefficients; i.e. we need two coefficients $(\alpha, \beta)$ per spectrum $(2 \times \mathrm{T}$ in total) in the original series that will tell us which linear combination of the first two eigenvectors $f_{1}^{\lambda_{j}}$ and $f_{2}^{\lambda_{j}}$ we need to represent a particular spectrum. That table is obtained from the following matrix operation (equivalent to the various scalar products of the spectra with the first two eigenvectors):

$$
C=\left(\begin{array}{ll}
\alpha_{1} & \beta_{1} \\
\alpha_{2} & \beta_{2} \\
\cdots & \cdots \\
\alpha_{T} & \beta_{T}
\end{array}\right)=\hat{M}\left(\begin{array}{cc}
f_{1}^{\lambda_{1}} & f_{2}^{\lambda_{1}} \\
f_{1}^{\lambda_{2}} & f_{2}^{\lambda_{2}} \\
\cdots & \cdots \\
f_{1}^{\lambda_{N}} & f_{2}^{\lambda_{N}}
\end{array}\right)
$$

This completes the standard PCA and gives us all the information needed to reconstruct the data: $(i)$ the coefficients matrix $C(T \times 2)$; (ii) the first two eigenvectors of the covariance matrix $f_{1}^{\lambda_{j}}$ and $f_{2}^{\lambda_{j}}($ each $1 \times N)$; and (iii) the original mean of each spectrum $\bar{I}_{t_{i}}(T \times 1)$. This represents a massive reduction in the amount of information that still captures the essential features of the data (i.e. from $T \times N$ in the original data matrix, to $3 T+2 N$ in the final arrays).

The $i$-th spectrum in the time series $\left(t_{i}\right)$ is reconstructed as a function of $\lambda_{j}$ as:

$$
I_{t_{i}}^{\lambda_{j}}=\alpha_{i} f_{1}^{\lambda_{j}}+\beta_{i} f_{2}^{\lambda_{j}}+\bar{I}_{t_{i}}
$$




\section{Modified PCA for BiASERS}

The particular application that we are interested in is the resolution of BiASERS spectra into their two components. In the PCA process outlined so far, it may well occur that the obtained principal components include contributions from both of the two analytes, in differing degrees, rather than as separate components. This is because the principal components are not based on the"physical meaning" of the signal, but rather on orthogonality and maximal variance conditions. This can be easily demonstrated visually. If we plot the eigenvectors obtained from standard PCA, we observe that often they include contributions from both molecules we are interested in. In coefficient space ( $\beta$ vs $\alpha$ ) we observe this as the principal two axes not being perpendicular (see Fig. A.4(a) and (b)). To tailor the PCA method to our purposes, we must apply a linear transformation in coefficient space to rotate and rescale the eigenvectors (and coefficients) to obtain the two components as the individual probes. 

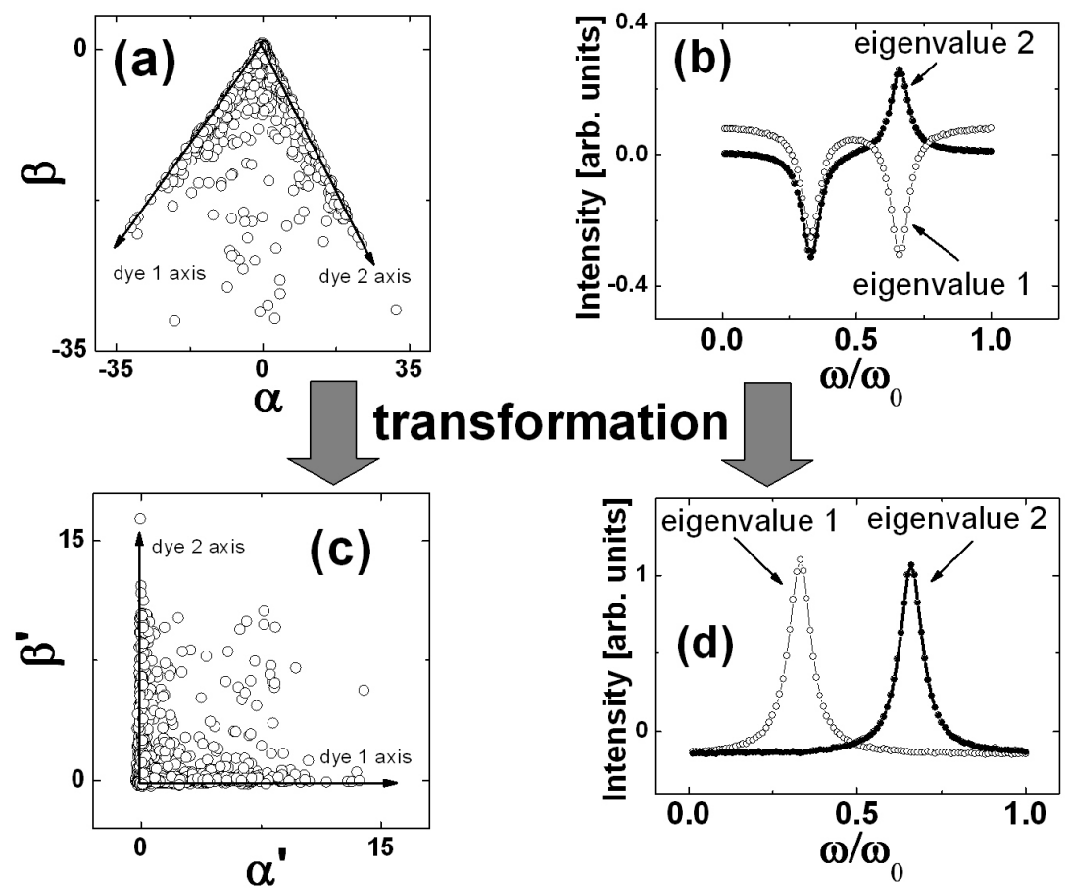

Figure A.4: (a) The two dimensional (2D) representation of the matrix $C$ in coefficient space; (b) the first two eigenvectors of the PCA analysis as a function of the reduced energy $\omega / \omega_{0}$. The first two eigenvectors of the covariance matrix do not necessarily separate the features we are trying to differentiate. In this example, both eigenvectors contain a mixture of signals from dye 1 at $\omega / \omega_{0}=0.33$ and from dye 2 at $\omega / \omega_{0}=0.66$. By applying a linear transformation to $C$ through the matrix $R$ defined in Eqn. A.27, we achieve the $2 \mathrm{D}$ representation of the coefficients shown in (c), together with the two (transformed) eigenvectors shown in (d). The principal axes for pure dye events are now perpendicular and the intensities are automatically re-scaled in the transformation (which would account for possible differences in the intrinsic cross-sections or concentrations of the dyes). The new (transformed) eigenvectors are now directly related to the independent contributions of the two dyes to the total signals, and a histogram of intensities can be obtained. 
We briefly outline this process below:

(i) We define two vectors $\vec{e}_{1}=n_{1}^{x} \vec{e}_{x}+n_{1}^{y} \vec{e}_{y}$ and $\vec{e}_{2}=n_{2}^{x} \vec{e}_{x}+n_{2}^{y} \vec{e}_{y}$ that are two unit vectors $\left(\left(\overrightarrow{e_{1}} \cdot \overrightarrow{e_{1}}=1\right.\right.$ and $\left.\left.\overrightarrow{e_{2}} \cdot \overrightarrow{e_{2}}=1\right)\right)$ along the principal directions representing "molecule 1 " events and "molecule 2 " events in coefficient space, as depicted schematically in Fig. A.4(a). This step is carried out manually by visual inspection of the plot. This approach is therefore not purely algorithmic, as would be a non-negative matrix factorization or independent component analysis approach, but is arguably more physical and intuitive.

(ii) We take the average spectrum (with zero mean intensity) and decompose it as a sum of the two main eigenvectors of the initial PCA, i.e:

$$
\left(\begin{array}{ll}
\alpha & \beta
\end{array}\right)=\left(\begin{array}{llll}
\left\langle\hat{I}^{\lambda_{1}}\right\rangle & \left\langle\hat{I}^{\lambda_{2}}\right\rangle & \ldots & \left\langle\hat{I}^{\lambda_{N}}\right\rangle
\end{array}\right)\left(\begin{array}{ll}
f_{1}^{\lambda_{1}} & f_{2}^{\lambda_{1}} \\
f_{1}^{\lambda_{2}} & f_{2}^{\lambda_{2}} \\
\ldots & \ldots \\
f_{1}^{\lambda_{N}} & f_{2}^{\lambda_{N}}
\end{array}\right) .
$$

We therefore obtain two coefficients $\alpha$ and $\beta$ (as we did for each individual spectrum in Eqn. (A.24)), which tell us how much of the first and second eigenvector we need to represent the average.

(iii) We now need to find the linear transformation $R$ that rotates $\vec{e}_{1}$ into $\vec{e}_{x}$, and $\vec{e}_{2}$ into $\vec{e}_{y}$, with possible scaling factors. These scaling factors must be chosen so that the transformed coefficients $\alpha$ and $\beta$ of the average spectrum are in the same ratio as the known dye concentrations $c_{1}$ and $c_{2}\left(c_{1}=c_{2}\right.$ in BiASERS assuming equivalent adsorption of the probes to the surface). One can show that $R$ must then be defined as [52]:

$$
R=\left(\begin{array}{ll}
k_{1} n_{1}^{x} & k_{2} n_{2}^{x} \\
k_{1} n_{1}^{y} & k_{2} n_{2}^{y}
\end{array}\right)^{-1}
$$

where

$$
\left(\begin{array}{l}
k_{1} \\
k_{2}
\end{array}\right)=\left(\begin{array}{ll}
c_{1} n_{1}^{x} & c_{2} n_{2}^{x} \\
c_{1} n_{1}^{y} & c_{2} n_{2}^{y}
\end{array}\right)^{-1}\left(\begin{array}{l}
\alpha \\
\beta
\end{array}\right)
$$


(iv) The transformation $R$ is applied to the coefficient matrix $C$ by standard matrix multiplication, thus defining a new table of coefficients $C^{\prime}=C\left({ }^{t} R\right)$.

(v) The first two eigenvectors must also be transformed accordingly into $g_{1}^{\lambda_{j}}$ and $g_{2}^{\lambda_{j}}$ as:

$$
\left(\begin{array}{c}
g_{1}^{\lambda_{j}} \\
\lambda_{j} \\
g_{2}
\end{array}\right)=\left({ }^{t} R\right)^{-1}\left(\begin{array}{c}
f_{1}^{\lambda_{j}} \\
f_{2}^{\lambda_{j}}
\end{array}\right) .
$$

Using this approach we obtain the separated eigenvectors and perpendicular coefficients as shown in Fig. A.4(c) and (d). We can now directly obtain the statistics on the relative proportions of "molecule 1" and "molecule 2" that contribute to each BiASERS spectrum and plot histograms as with other data analysis techniques. In practise, this analysis is done using in-house Matlab software with a handy visual GUI interface (Courtesy of Dr M. Meyer).

\section{Benefits and drawbacks of the MPCA approach}

The MPCA technique is ideally suited to the analysis of large numbers of spectra as it is a means of obtaining the most important peaks without having to actually fit the data, thus removing any consideration of the suitability of the function for fitting. Additionally, there is no need to obtain experimental reference spectra as is the case in linear deconvolution. The distinct spectral elements simply "appear" due to their presence in the data, even if they are often mixed. The drawback with this technique is that the data must be of sufficient quality (and quantity) to reliably obtain the features of each molecule. If there are large numbers of spurious signals in the region of interest, or if the signals are minimally above the noise, it will not be possible to obtain two distinct eigenvectors. For example, cosmic rays incident on the CCD detectors can sometimes be a problem, because within the small spectral windows normally analyzed they provide a large variance and can therefore be selected as one of the main eigenvectors. 


\section{Appendix B}

\section{RAW SPECTRAL DATA}

This section provides the raw data for characterization of the synthetic probes used in Chapter 6, including mass spectroscopy, ${ }^{1} \mathrm{H}-\mathrm{NMR}$, and ${ }^{13} \mathrm{C}-\mathrm{NMR}$. For summaries of the NMR, mass spectroscopy, and synthetic details see Section 3.5. 


\section{Natural Rh6M}

\section{Elemental Composition Report}

\section{Single Mass Analysis}

Tolerance $=5.0 \mathrm{mDa} / \mathrm{DBE}: \min =-1.5, \max =50.0$

Selected filters: None

Monoisotopic Mass, Even Electron lons

90 formula(e) evaluated with 1 results within limits (up to 50 closest results for each mass)

Elements Used:

C: $0-50 \quad H: 0-100 \quad N: 2-2 \quad 0: 0-50$

EBRHGM 30 (0.513) AM (Cen,4, 80.00, Ar,10000.0,413.27,0.70); Sm (SG, 1x7.00); Sb $(15,10.00) ; \mathrm{Cm}(2: 30)$

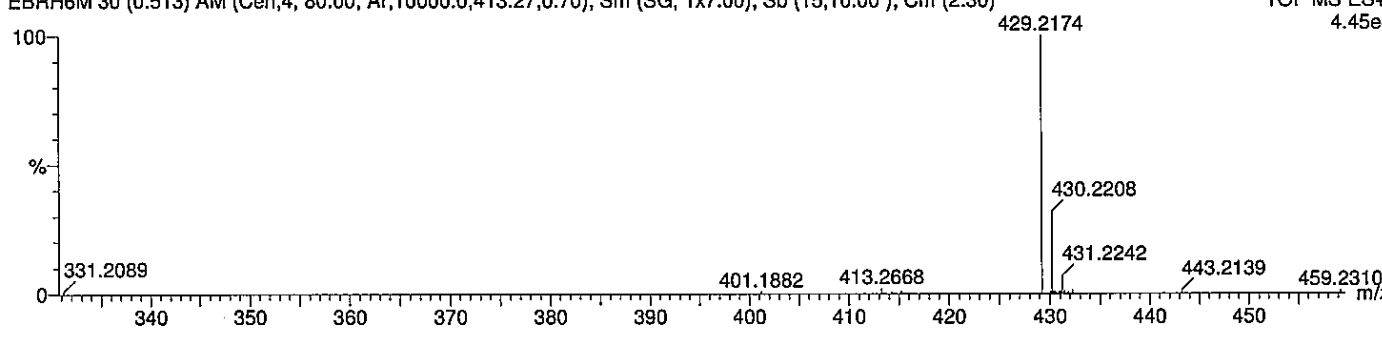

Minimum

Maximum:

$-1.5$

Mass Calc. Mass mDa PPM

$429.2174 \quad 429.2178$

$-0.4 \quad-0.9$

DBE $\quad$ i-FIT

Formula

429.2178

$-0.4 \quad-0.9$

14.5

43.0

$\begin{array}{lll}\mathrm{C} 27 & \mathrm{H} 29 & \mathrm{~N} 2 \quad \mathrm{O}\end{array}$ 


\section{d4-Rh6M}

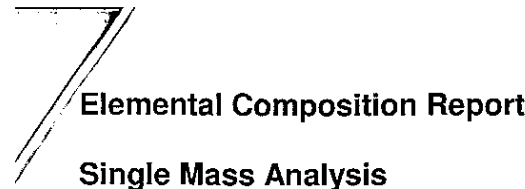

Tolerance $=5.0 \mathrm{mDa} / \mathrm{DBE}: \min =-1.5, \max =50.0$

Selected filters: None

Monoisotopic Mass, Even Electron lons

206 formula(e) evaluated with 1 results within limits (up to 50 closest results for each mass)

Elements Used:

$\begin{array}{llll}C: 0-50 & \mathrm{~N}: 2-2 & 0: 0-50 \quad 1 \mathrm{H}: 0-50 \quad 2 \mathrm{H}: 4-4\end{array}$

Evan Blackie

EBD4RHGM 25 (0.428) AM (Cen,4, 80.00, Ar, 10000.0,413.27,0.70); Sm (SG, 1x7.00); Sb (15,10.00); Cm (2:30)

$1007 \quad 433.2425$
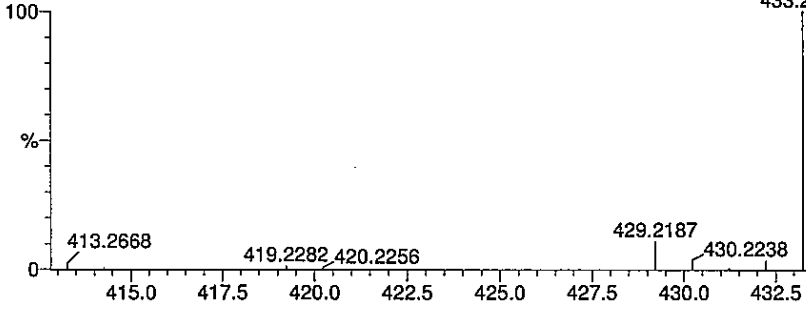

19-Nov-2007

Minimum:

Maximum:

5.0

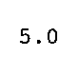

$-1.5$

Mass

Calc. Mass

$\mathrm{mDa}$

PPM

$433.2425 \quad 433.2429$

$\begin{array}{ll}-0.4 & -0.9\end{array}$

14.5

i-FIT

Formula 


\section{Natural Rh6M ${ }^{1}$ H-NMR}

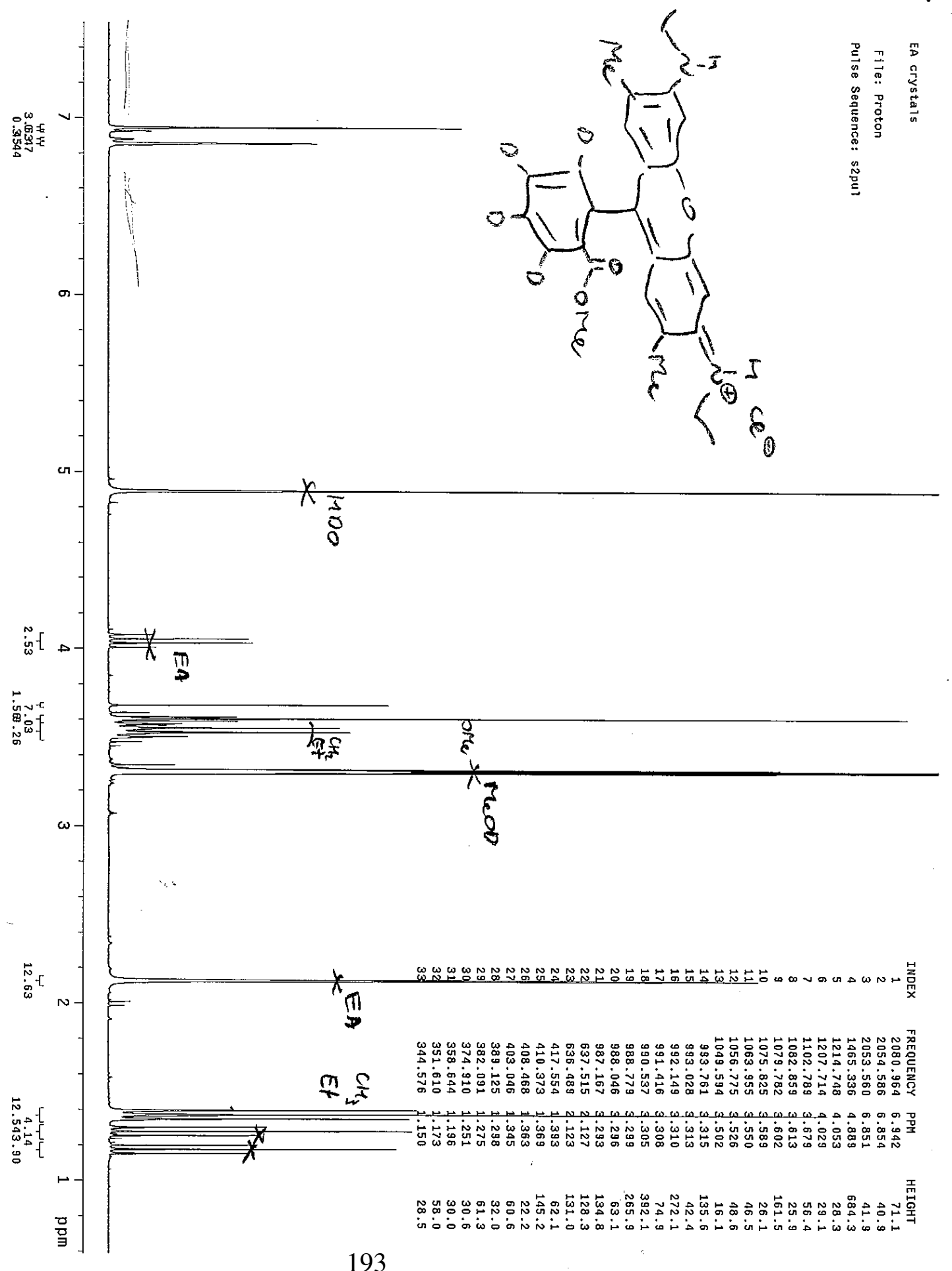




\section{d4-Rh6M ${ }^{1}$ H-NMR}

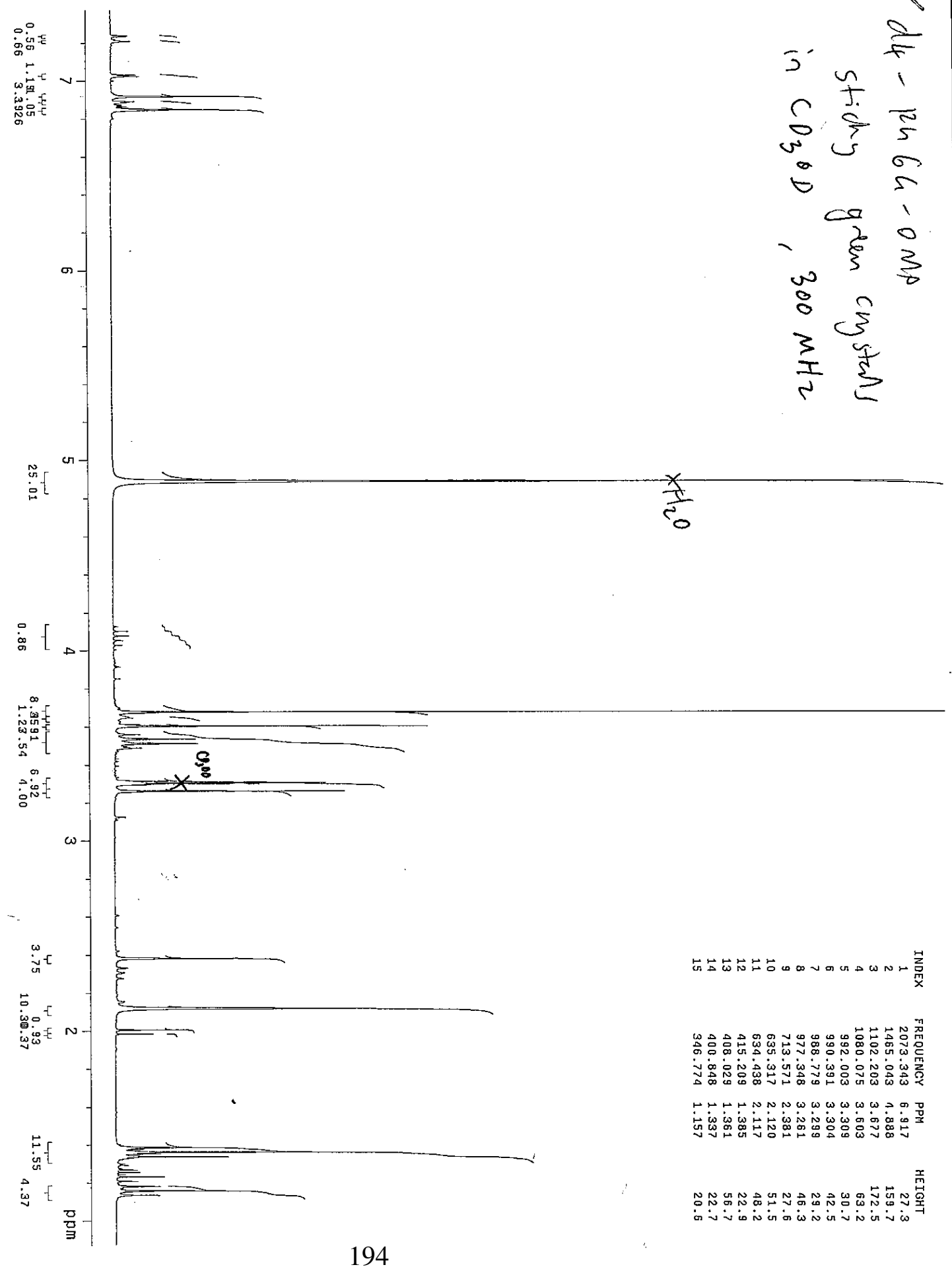




\section{Natural Rh6M ${ }^{13}$ C-NMR}

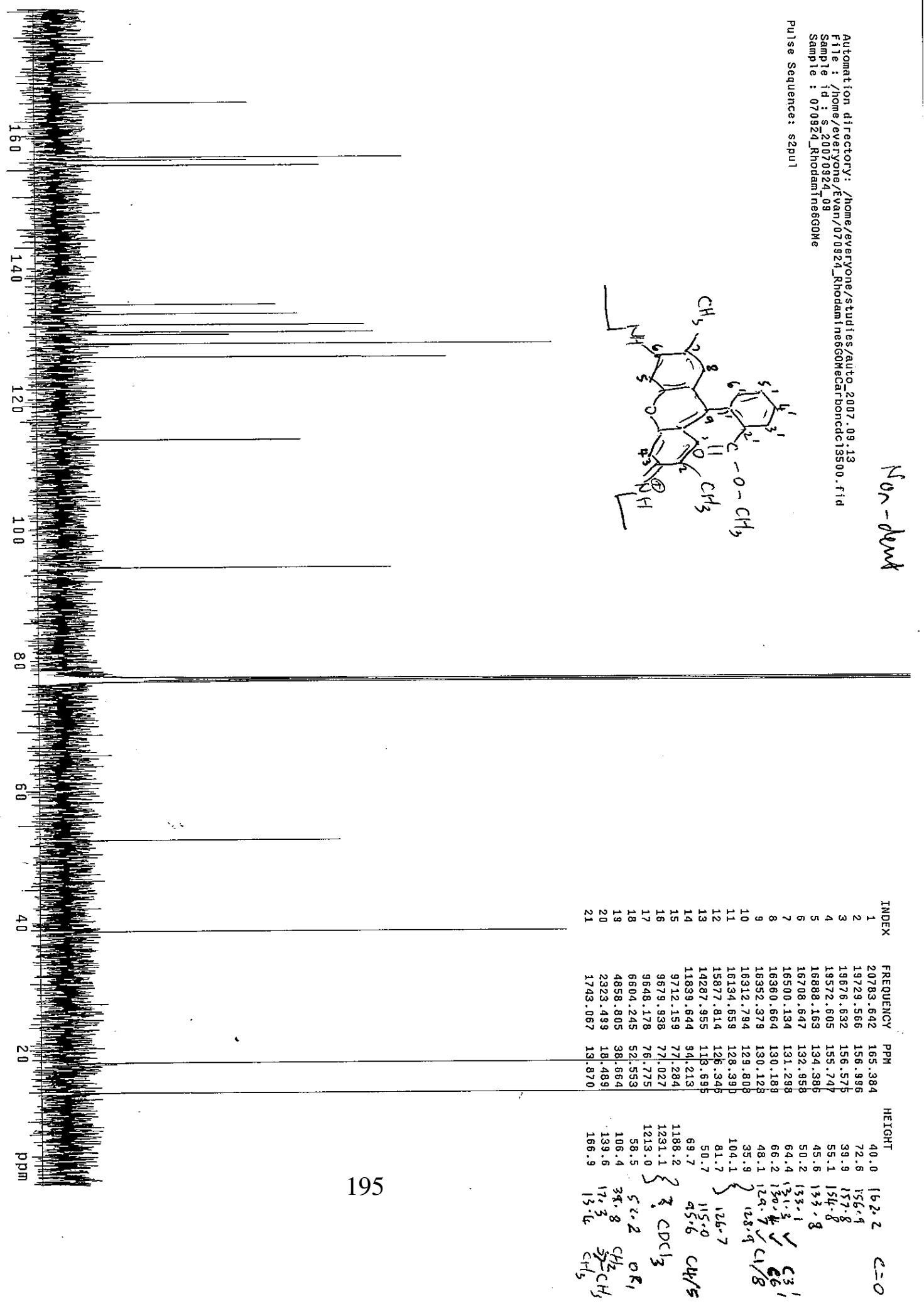




\section{d4-Rh6M ${ }^{13}$ C-NMR}

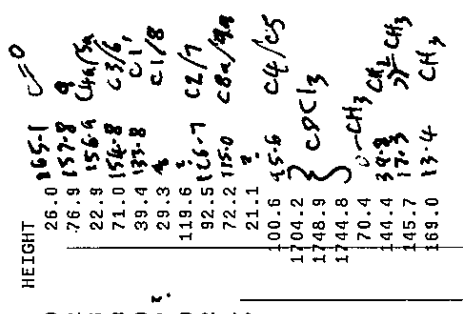

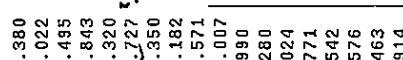

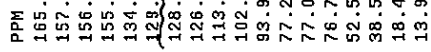

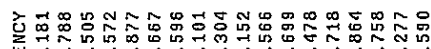

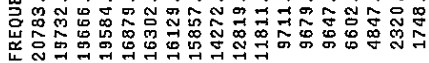

产

$\frac{5}{3}$

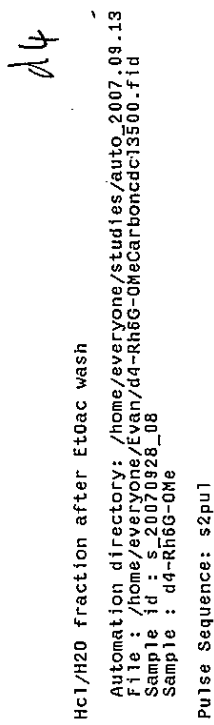

196

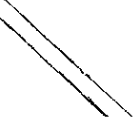




\section{Appendix C}

\section{PUBLICATIONS}

For the interested reader, here are the headers of papers published during the course of the thesis.

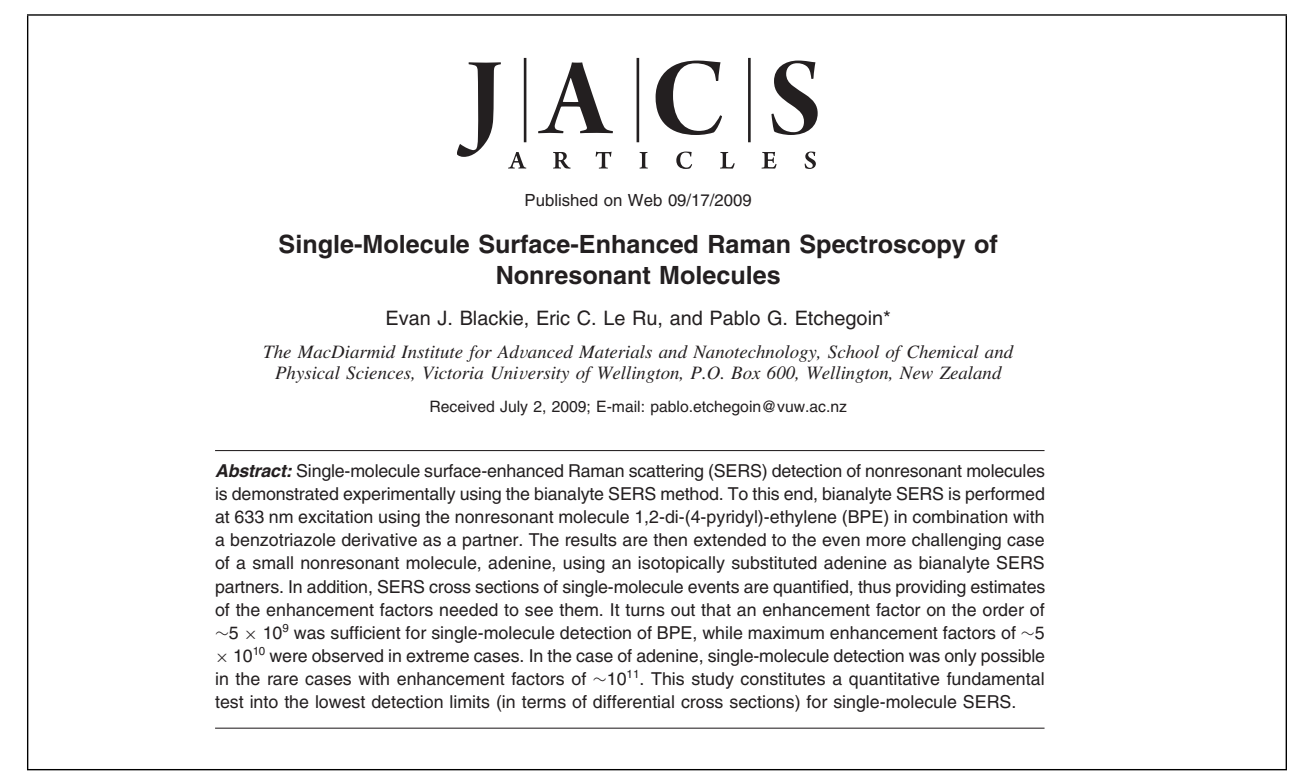




\section{PHIF JOLRNAL OF

Experimental Verification of the SERS Electromagnetic Model beyond the $|E|^{4}$ Approximation: Polarization Effects

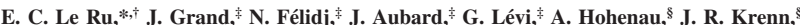
E. Blackie, ${ }^{\dagger}$ and P. G. Etchegoin

The MacDiarmid Institute for Advanced Materials and Nanotechnology, School of Chemical and Physical Sciences, Victoria University of Wellington, P.O. Box 600, Wellington, New Zealand, Laboratoire ITODYS, Universite Paris 7, Denis Diderol, CNRS UMR 7086, I Rue Gay de la Brosse, F, 75005 Paris, France, an

Received: March 13, 2008

The failure of the so-called $|E|^{4}$ approximation of the surface-enhanced Raman scattering (SERS) electromagnetic (EM) enhancement factor is demonstrated experimentally using arrays of highly uniform gold nanoparticles specially designed for this purpose. This is first demonstrated for unpolarized detection, and the interpretation of the results then becomes evident by studying the case of polarized detection. These results provide, at the same time, a clear experimental verification of the generalized EM theory of SERS beyond the $|E|$ approximation (Chem. Phys. Lett. 2006, 423, 63). A nontrivial consequence of these concepts is the almost complete polarization rotation of the SERS signal with respect to the incident polarization. This occurs for a prolate nanoparticle with plasmon resonances peaking on either side of the main spectral range covered by the Raman spectrum of the probe. Finally, we discuss how such experiments can be used to extract some information about the molecular adsorption orientation of the probe.

JOURNAL OF RAMAN SPECTROSCOPY

(www interscience wiley com) DOI. $10.1002 /$ jrs 1945

\section{Advanced aspects of electromagnetic SERS enhancement factors at a hot spot}

E. C. Le Ru, ${ }^{*}$ M. Meyer, E. Blackie and P. G. Etchegoin

The MacDiarmid Institute for Advanced Materials and Nanotechnology, School of Chemical and Physical Sciences, Victoria University of Wellington Wellington, New Zealand

Received 18 September 2007; Accepted 2 December 2007

In this paper, we discuss some advanced theoretical aspects of electromagnetic enhancement factors ( $\mathrm{EFs})$ in sur coles (SSR obsenable experis property that hey can appropriate tools are developed here to make predictions beyond this approximation in the case of a SERS hot spot. In addition, theoretical estimates of different types of (previously defined) EFs are provided and their origins discussed for the typical example of a SERS substrate dominated by SERS hot spots. Finally, experimental measurements of SERS depolarization ratios are presented to support the theoretical predictions. Copyright $\odot 2008$ John Wiley \& Sons, Ltd. 
Bi-analyte SERS with isotopically edited dyes

E. Blackie, ${ }^{, a b}$ E. C. Le Ru, ${ }^{a b}$ M. Meyer ${ }^{a b}$ M. Timmer, ${ }^{b c}$ B. Burkett, ${ }^{b}$

P. Northcote ${ }^{b}$ and P. G. Etchegoin ${ }^{a b}$

Received 4th March 2008, Accepted 21st April 2008

First published as an Advance Article on the web 3rd June 2008

DOI: $10.1039 / \mathrm{b803738 \textrm {h }}$

Isotopically substituted rhodamine dyes provide ideal probes for the study of single-molecule

surface enhanced Raman scattering (SM-SERS) events through multiple-analyte techniques.

Isotopic editing should, in principle, provide probes that have identical chemical properties (and

surface chemistries); while exhibiting at the same time distinct Raman features which enable us to

identify single-molecule SERS events. We present here a specific example of two-analyte

SM-SERS based on the isotopic substitution of a methyl ester rhodamine dye. The dyes are

carefully characterized (in both standard and SERS conditions) to confirm experimentally their

similar chemical properties. We then demonstrate their utility for bi-analyte SERS (BiASERS)

experiments and, as an example, highlight the transition from a single, to a few, to many

molecules in the statistics of SM-SERS signal.

Technical Notes

Statistics of Single-Molecule Surface Enhanced

Raman Scattering Signals: Fluctuation Analysis with Multiple Analyte Techniques

P. G. Etchegoin,* M. Meyer, E. Blackie, and E. C. Le Rü

The MacDiarmid Institute for Advanced Materials and Nanotechnology, School of Chemical and Physical Sciences, Victoria University of Wellington, P.O. Box 600 Wellington, New Zealand

The mathematical background, based on a variation of the principal component analysis (PCA) method, is developed for the understanding of fluctuating multiple analyte single-molecule (SM) surface enhanced Raman scattering (SERS) signals; with emphasis on the bianalyte SERS technique developed recently. The method and its significance are presented to provide a systematic framework with which several aspects of the statistics of SM-SERS signals can be analyzed in general. We also apply the method to a concrete example of bianalyte statistics in silver colloidal solutions and discuss related topics ars experimental issues and the interpretation of singe molec SEPS data. that purpose by other authors, 5,6 but a few questions remain; among them: (i) how much can we obtain or learn from the statistics of single-molecule events in BiASERS? and (ii) is the a systematic and reliable method for the study of single-molecule statistics in SERS from two or many analytes?

It is the purpose of this paper to develop further the ideas and tools needed to understand single-molecule fluctuations in SERS with multiple analytes. To this end, we shall develop an analysi tool based on a variation of the principal component analysis (PCA) method. We shall assume no previous exposure to the $\mathrm{PC}$ method and, therefore, we shall explain the relevant steps in some detail in the Supporting Information companion material to this paper, making emphasis on the physical meaning of the results. 
Surface Enhanced Raman Scattering Enhancement Factors: A Comprehensive Study

E. C. Le Ru,* E. Blackie, M. Meyer, and P. G. Etchegoin

The MacDiarmid Institute for Advanced Materials and Nanotechnology, School of Chemical and Physical

Sciences, Victoria University of Wellington, PO Box 600, Wellington 6140, New Zealand

Received: December 21, 2006; In Final Form: July 17, 2007

This paper presents an in-depth study of Surface Enhanced Raman Scattering (SERS) enhancement factors (EFs) and cross-sections, including several issues often overlooked. In particular, various possible rigorous definitions of the SERS EFs are introduced and discussed in the context of SERS applications, such as analytical of the non-SERS coss-section of the pres under constion results for the nOD-SERS cross-cetions of representive SERS probs a

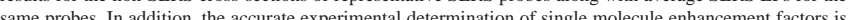
tackled with two recently developed techninues, namely: bi antyt SERS (BiASERS) and temperato dependent SERS vibrational pumping. We dem, figure of $10^{14}$ often claimed in the literature, are sufficient for the observation of single molecule SERS signals, with maximum single molecule EFs typically on the order of $\sim 10^{10}$. 


\section{References}

[1] Long, D. A. The Raman Effect, A Unified Treatment of the Theory of Raman Scattering by Molecules. John Wiley and Sons Ltd., Chichester, (2002).

[2] Le Ru, E. C. and Etchegoin, P. G. Principles of Surface Enhanced Raman Spectroscopy and Related Plasmonic Effects. Elsevier, Amsterdam, (2009).

[3] Ferraro, J. R., Nakamoto, K., and Brown, C. W. Introductory Raman Spectroscopy; 2nd ed.

[4] Smekal, A. Naturwissenschaften 11, 873 (1923).

[5] Raman, C. V. and Krishnan, K. S. Nature 121, 501 (1928).

[6] Schroetter, H. W. and Kloeckner, H. W. Raman spectroscopy of gases and liquids. Springer-Verlag, Berlin, (1979).

[7] Schrader, B. and Moore, D. S. Pure and Appl. Chem. 69, 1451 (1997).

[8] Placzek, G. Z. Physik 70, 84 (1931).

[9] Neugebauer, J., Reiher, M., Kind, C., and Heiss, B. A. J. Computational Chem. 23, 895 (2002).

[10] Hayes, W. and Loudon, R. Scattering of Light by Crystals. Wiley, New York, (1978).

[11] Boyd, R. W. Nonlinear Optics. Academic Press, New York, (1992). 
[12] Nogueira, F., Castro, A., and Marques, M. A. L. A Tutorial on Density Functional Theory, volume 620 of Lecture Notes in Physics, chapter 6, 218. Springer Berlin / Heidelberg, Berlin (2003).

[13] Dreizler, R. M. and Gross, E. K. U. Density Functional Theory: An Approach to the Quantum Many-Body Problem. Springer-Verlag, Berlin, (1990).

[14] Frisch, M. J., Trucks, G. W., Schlegel, H. B., Scuseria, G. E., Robb, M. A., Cheeseman, J. R., Montgomery, J. A., Jr., Vreven, T., Kudin, K. N., Burant, J. C., Millam, J. M., Iyengar, S. S., Tomasi, J., Barone, V., Mennucci, B., Cossi, M., Scalmani, G., Rega, N., Petersson, G. A., Nakatsuji, H., Hada, M., Ehara, M., Toyota, K., Fukuda, R., Hasegawa, J., Ishida, M., Nakajima, T., Honda, Y., Kitao, O., Nakai, H., Klene, M., Li, X., Knox, J. E., Hratchian, H. P., Cross, J. B., Adamo, C., Jaramillo, J., Gomperts, R., Stratmann, R. E., Yazyev, O., Austin, A. J., Cammi, R., Pomelli, C., Ochterski, J. W., Ayala, P. Y., Morokuma, K., Voth, G. A., Salvador, P., Dannenberg, J. J., Zakrzewski, V. G., Dapprich, S., Daniels, A. D., Strain, M. C., Farkas, O., Malick, D. K., Rabuck, A. D., Raghavachari, K., Foresman, J. B., Ortiz, J. V., Cui, Q., Baboul, A. G., Clifford, S., Cioslowski, J., Stefanov, B. B., Liu, G., Liashenko, A., Piskorz, P., Komaromi, I., Martin, R. L., Fox, D. J., Keith, T., Al-Laham, M. A., Peng, C. Y., Nanayakkara, A., Challacombe, M., Gill, P. M. W., Johnson, B., Chen, W., Wong, M. W., Gonzalez, C., and Pople, J. A. Technical report, Gaussian, Inc., Pittsburgh PA, (2003).

[15] http://www.msg.ameslab.gov/GAMESS/.

[16] Meyer, M. Thesis, Victoria of University, (2009). http://hdl.handle.net/10063/990.

[17] El-Azhary, A. A. and Suter, H. U. J. Phys. Chem. 99, 12751 (1995).

[18] Fleischmann, M., Hendra, P. J., and McQuillan, A. J. Chem. Phys. Lett. 26, 163 (1974).

[19] Jeanmaire, D. L. and Duyne, R. P. V. J. Electroanal. Chem. 84, 1 (1977). 
[20] Albrecht, M. G. and Creighton, J. A. J. Am. Chem. 99, 5215 (1977).

[21] Moskovits, M. Rev. Mod. Phys. 57, 783 (1985).

[22] Aroca, R. F. Surface-Enhanced Vibrational Spectroscopy. John Wiley and Sons, Chichester, (2006).

[23] Le Ru, E. C. and Etchegoin, P. G. Chem. Phys. Lett. 423, 63 (2006).

[24] Bohren, C. F. and Huffman, D. R. Absorption and Scattering of Light by Small Particles. Wiley, New York, (1983).

[25] Asano, S. and Yamamoto, G. Appl. Optics 14, 29 (1975).

[26] Gerardy, J. M. and Ausloos, M. Phys. Rev. B 25, 4204 (1982).

[27] G. C. Schatz, M.A. Young, R. V. D. Surface-Enhanced Raman Scattering: Physics and Applications (Topics in Applied Physics Vol. 103), 19. Springer, Berlin (2006).

[28] Johansson, P., Xu, H., and Käll, M. Phys. Rev. B 72, 035427 (2005).

[29] Xu, H., Aizpurua, J., Käll, M., and Apell, P. Phys. Rev. E 62, 4318 (2000).

[30] Le Ru, E. C. and Etchegoin, P. G. J. Chem. Phys. 130, 181101 (2009).

[31] Lombardi, J. R., Birke, R. L., Lu, T., and Xu, J. J. Chem. Phys. 84, 4174 (1986).

[32] Otto, A. J. Raman Spectrosc. 33, 593 (2002).

[33] Rubim, J. and Sala", O. Journal of Molecular Structure 145, 157 - 172 (1986).

[34] Natan, M. Faraday Disc. 132, 321 (2006).

[35] Le Ru, E. C. and Etchegoin, P. G. Phys. Rev. Lett. 97, 199701 (2006).

[36] Kneipp, K., Wang, Y., Kneipp, H., Perelman, L. T., Itzkan, I., Dasari, R. R., and Feld, M. S. Phys. Rev. Lett. 78, 1667 (1997). 
[37] Campion, A., Ivanecky, J. E., Child, C. M., and Foster, M. J. Am. Chem. Soc. 117, 11807 (1995).

[38] King, F. W., Duyne, R. P. V., and Schatz, G. C. J. Chem. Phys. 69, 4472 (1978).

[39] Efrima, S. and Metiu, H. J. Chem. Phys. 70, 1602 (1979).

[40] Nie, S. and Emory, S. R. Science 275, 1102 (1997).

[41] Le Ru, E. C., Meyer, M., and Etchegoin, P. G. J. Phys. Chem. B 110, 1944 (2006).

[42] Etchegoin, P. G., Meyer, M., and Le Ru, E. C. Phys. Chem. Chem. Phys. 9, 3006 (2007).

[43] Le Ru, E. C., Etchegoin, P. G., and Meyer, M. J. Chem. Phys. 125, 204701 (2006).

[44] Etchegoin, P. G., Le Ru, E. C., and Meyer, M. J. Chem. Phys. 127, 189901 (2007).

[45] Emory, S. R., Nie, S., Kneipp, K., and Harrison, G. R. Chimia 53, 35 (1999).

[46] Dickson, R. M., Cubitt, A. B., Tsien, R. Y., and Moerner, W. E. Nature (London) 388, 355 (1997).

[47] Trautman, J. K., Macklin, J. J., Brus, L. E., and Betzig, E. Nature (London) 369, 40 (1994).

[48] Michaels, A. M., Nirmal, M., and Brus, L. E. J. Am. Chem. Soc. 121, 9932 (1999).

[49] Futamata, M. Faraday Disc. 132, 45 (2006).

[50] Weiss, A. and Haran, G. J. Phys. Chem. B 105, 12348 (2001).

[51] Maruyama, Y., Ishikawa, M., and Futamata, M. J. Phys. Chem. B 108, 673 (2004). 
[52] Etchegoin, P. G., Meyer, M., Blackie, E., and Le Ru, E. C. Anal. Chem. 79, 8411 (2007).

[53] Sawai, Y., Takimoto, B., Nabika, H., Ajito, K., and Murakoshi, K. J. Am. Chem. Soc. 129, 1658 (2007).

[54] Goulet, P. J. G. and Aroca, R. F. Anal. Chem. 79, 2728 (2007).

[55] Santos, D. P. d., Andrade, G. F. S., Temperini, M. L. A., and Brolo, A. G. J. Phys. Chem. C 113, 17737 (2009).

[56] Le Ru, E. C., Blackie, E., Meyer, M., and Etchegoin, P. G. J. Chem. Phys. C 111, 13794 (2007).

[57] Dieringer, J. A., Lettan II, R. B., Scheidt, K. A., and Van Duyne, R. P. J. Am. Chem. Soc. 129, 16249 (2007).

[58] McFarland, A. D., Young, M. A., Dieringer, J. A., and Duyne, R. P. V. J. Phys. Chem. B 109, 11279 (2005).

[59] Hildebrandt, P. and Stockburger, M. J. Phys. Chem. 88, 5935 (1984).

[60] Félidj, N., Aubard, J., Lévi, G., Krenn, J. R., Salerno, M., Schider, G., Lamprecht, B., Leitner, A., and Aussenegg, F. R. Phys. Rev. B 65, 075419 (2002).

[61] Cai, W. B., Ren, B., Li, X. Q., She, C. X., Liu, F. M., Cai, X. W., and Tian, Z. Q. Surf. Sci. 406, 9 (1998).

[62] Kneipp, K., Wang, Y., Kneipp, H., Itzkan, I., Dasari, R. R., and Feld, M. S. Phys. Rev. Lett. 76, 2444 (1996).

[63] Brolo, A. G., Sanderson, A. C., and Smith, A. P. Phys. Rev. B 69, 045424 (2004).

[64] Haslett, T. L., Tay, L., and Moskovits, M. J. Chem. Phys. 113, 1641 (2000).

[65] Maher, R. C., Cohen, L. F., Le Ru, E. C., and Etchegoin, P. G. Faraday Discuss. 132, 77 (2006). 
[66] Le Ru, E. C. and Etchegoin, P. G. Faraday Discuss. 132, 63 (2006).

[67] Maher, R. C., Cohen, L. F., Gallop, J. C., Le Ru, E. C., and Etchegoin, P. G. J. Phys. Chem. B 110, 6797 (2006).

[68] Maher, R. C., Etchegoin, P. G., Le Ru, E. C., and Cohen, L. F. J. Phys. Chem. B 110, 11757 (2006).

[69] Maher, R. C., Cohen, L. F., Le Ru, E. C., and Etchegoin, P. G. J. Phys. Chem. B 110, 19469 (2006).

[70] Maher, R. C., Hou, J., Cohen, L. F., Ru, E. C. L., Hadfield, J. M., Harvey, J. E., Etchegoin, P. G., Liu, F. M., Green, M., Brown, R. J. C., and Milton, M. J. T. J. Chem. Phys. 123, 084702 (2005).

[71] Demtroder, W. Laser Spectroscopy. Springer, Berlin, (2002).

[72] Galloway, C. M., Etchegoin, P. G., and Le Ru, E. C. Phys. Rev. Lett. 103, 063003 (2009).

[73] Lee, P. and Meisel, D. J. Phys. Chem. 86, 3391 (1982).

[74] Meyer, M., Le Ru, E. C., and Etchegoin, P. G. J. Phys. Chem. B 110, 6040 (2006).

[75] Creighton, J. A., Blatchford, C. G., and Creighton, M. G. J. Chem. Soc.: Faraday Trans. 2, 790 (1979).

[76] http://www.gaussian.com/.

[77] Becke, A. D. J. Chem. Phys. 98, 5648 (1993).

[78] Lee, C., Yang, W., and Parr, R. G. Phys. Rev. B 37, 785789 (1988).

[79] Abudara. Eur. Pat. Appl., (1992). Patent no: EP0468821.

[80] Lal, S., Grady, N. K., Goodrich, G. P., and Halas, N. J. Nano Lett. 6, 2338 (2006).

[81] Jensen, L. and Schatz, G. C. J. Phys. Chem. A 110, 5973 (2006). 
[82] Sarkar, J., Chowdhury, J., Pal, P., and Talapatra, G. B. Vib. Spectrosc. 41, 90 (2006).

[83] Naumov, S., Kapoor, S., Thomas, S., Venkateswaran, S., and Mukherjee, T. J. Mol. Structure (Theochem) 685, 127 (2004).

[84] Pagliai, M., Bellucci, L., Muniz-Miranda, M., Cardini, G., and Schettino, V. Phys. Chem. Chem. Phys. 8, 171 (2006).

[85] Pagliai, M., Cardini, G., Righini, R., and Schettino, V. J. Chem. Phys. 119, 6655 (2003).

[86] Watanabe, H., Hayazawa, N., Inouye, Y., and Kawata, S. J. Phys. Chem. B 109, 5012 (2005).

[87] Kato, Y. and Takuma, H. J. Chem. Phys. 54, 5398 (1971).

[88] Graham, D., McLaughlin, C., McAnally, G., Jones, J. C., White, P. C., and Smith, W. E. Chem. Comm. , 1187 (1998).

[89] Baptista, M.S., I. G. J. Phys. Chem. B 102, 4678 (1998).

[90] Chowdhury, J., Pal, P., Ghosh, M., and Misra, T. N. J. Colloid Interface Sci. 235 (2001).

[91] Angeloni, L., Smulevich, G., and Marzocchi, M. P. J. Raman Spectrosc. 8, 305 (1979).

[92] Mock, J. J., Barbic, M., Smith, D. R., Schultz, D. A., and Schultz, S. J. Chem. Phys. 116, 6755 (2002).

[93] Le Ru, E. C., Dalley, M., and Etchegoin, P. G. Current Appl. Phys. 6, 411 (2006).

[94] Moskovits, M. J. Chem. Phys. 77, 4408 (1982).

[95] Thomas, S., Venkateswaran, S., Kapoor, S., Cunha, R. D., and Mukherjee, T. Spectrochimica Acta 60, 25 (2004). 
[96] Gerratt, J. and Mills, I. M. J. Chem. Phys. 49, 1730 (1968).

[97] Domke, K. F. and Pettinger, B. Phys. Rev. B 75, 236401 (2007).

[98] Kneipp, K. and Kneipp, H. Surface-Enhanced Raman Scattering: Physics and Applications (Topics in Applied Physics Vol. 103), 183. Springer, Berlin (2006).

[99] Zhang, D., Xie, Y., Deb, S. K., Davisson, V. J., and Ben-Amotz, D. Anal. Chem. 77, 3563 (2005).

[100] Eur. Pat. Appl., (1887). Patent no: DE48731.

[101] Kubin, R. F. and Fletcher, A. N. J. Lumin. 27, 455 (1983).

[102] Lloyd, D. and Scott, I. R. Anal Biochem 128 (1983).

[103] Vosgroene, T. and Meixner, A. J. ChemPhysChem 6, 154 (2005).

[104] McMurry, J. Organic chemistry, 4th ed. Brooks/Cole Publishing Company, California, (1996).

[105] Ramos, S. S., Vilhena, A. F., Santos, L., and Almeida, P. Magnetic Resonance in Chemistry 38, 475 (2000).

[106] Papp, C., Fuhrmann, B., Tränkenschuh, B., Denecke, R., and Steinrück, H. P. Chem. Phys. Lett. 442, 176 (2007).

[107] McCullagh, James V.; Daggett, K. A. J. Chem. Educ. 84, 1799 (2007).

[108] Deb, S. K., Davis, B., Ben-Amotz, D., and Davisson, V. J. Appl. Spectrosc. 62, 1001 (2008).

[109] Doering, W. E. and Nie, S. J. Phys. Chem. B 106, 311 (2002).

[110] Etchegoin, P. G. and Le Ru, E. C. Phys. Chem. Chem. Phys. 10, 6079 (2008).

[111] Maruyama, Y., Ishikawa, M., and Futamata, M. Chem. Lett. 30, 834 (2001). 
[112] Kneipp, K., Kneipp, H., Kartha, V. B., Manoharan, R., Deinum, G., Itzkan, I., Dasari, R. R., and Feld, M. S. Phys. Rev. E 57, R6281 (2001).

[113] Maher, R. C., Galloway, C. M., Le Ru, E. C., Cohen, L. F., and Etchegoin, P. G. Chem. Soc. Rev. 37, 965 (2008).

[114] Zhang, X., Yonzon, C., Young, M., Stuart, D., and Van Duyne, R. Nanobiotechnology, IEE Proceedings - 152, 195 (2005).

[115] Vo-Dinh, T. Sens. Actuators, B B29, 183 (1995).

[116] Ryder, A. G. Curr. Opin. Chem. Biol. 9, 489 (2005).

[117] Sedlacek, A. J., Christesen, S. D., Vo-Dinh, T., and Combs, R. J., editors. Chemical and Biological Point Sensors for Homeland Defense II, volume 5585 of Society of Photo-Optical Instrumentation Engineers (SPIE) Conference Series. (2004).

[118] Yeo, B.-S., Schmid, T., Zhang, W., and Zenobi, R. Appl. Spectrosc. 62, 708 (2008).

[119] Kudelski, A. and Pettinger, B. Chem. Phys. Lett. 321, 356 (2000).

[120] Brolo, A. G., Irish, D. E., and Smith, B. D. J. Mol. Struct. 405, 29 (1997).

[121] Snchez-Corts, S. and Garca-Ramos, J. V. Spectrochim. Acta, Part A 29, 365 (1998).

[122] Bjerneld, E. J., Svedberg, F., Johansson, P., and Käll, M. J. Phys. Chem. A 108, 4187 (2004).

[123] Fang, Y., Seong, N.-H., and Dlott, D. D. Science 321, 388 (2008).

[124] McMahon, J. J. and Babcock, G. T. Spectrochim. Acta, Part A 38A, 1115 (1982).

[125] Felidj, N., Aubard, J., Levi, G., Krenn, J. R., Hohenau, A., Schider, G., Leitner, A., and Aussenegg, F. R. Appl. Phys. Lett. 82, 3095 (2003). 
[126] Aroca, R., Corio, P., and Rubim, J. C. Ann. Chim. 87, 1 (1997).

[127] Freeman, R. G., Bright, R. M., Hommer, M. B., and Natan, M. J. Raman Spectrosc. 30, 733 (1999).

[128] Rau, H. and Lueddecke, E. J. Am. Chem. Soc. 104, 1616 (1982).

[129] Chang, C.-W., Y-C, L., T-T, W., and E., D. J. Am. Chem. Soc. 126, 10109 (2004).

[130] Satzger, H., Root, C., and Braun, M. J. Phys. Chem. A 108, 6265 (2004).

[131] Yoon, J. and Yoon, S. Bull. Korean Chem. Soc. 30, 2877 (2009).

[132] Le Ru, E. C., Meyer, M., Blackie, E., and Etchegoin, P. G. J. Raman Spectrosc. 39, 1127 (2008).

[133] Blackie, E., Le Ru, E. C., Meyer, M., Timmer, M., Burkett, B., Northcote, P., and Etchegoin, P. G. Phys. Chem. Chem. Phys. 10, 4147 (2008).

[134] Bernd, G. and McNaughton, D. J. Phys. Chem. C 106, 1461 (2002).

[135] Li, K. R., Stockman, M. I., and Bergman, D. J. Phys. Rev. Lett. 91, 227402 (2003).

[136] Etchegoin, P. G., Galloway, C., and Le Ru, E. C. Phys. Chem. Chem. Phys. 8, 2624 (2006).

[137] Tian, Z. Q. Faraday Discuss. 132, 309 (2006).

[138] Gu, G. H. and Suh, J. S. J. Phys. Chem. A 113, 8529 (2009).

[139] Kneipp, J., Kneipp, H., and Kneipp, K. Chem. Soc. Rev. 37, 1052 (2008).

[140] Otto, A. J. Raman Spectrosc. 37, 937 (2006).

[141] Golab, J. T., Sprague, J. R., Carron, K. T., Schatz, G. C., and Van Duyne, R. P. J. Chem. Phys. 88, 7942 (1988).

[142] Novotny, L. and Hecht, B. Principles of nano-optics. Cambridge University Press, Cambridge, (2006). 
[143] Saleh, B. E. A. and Teich, M. C. Fundamentals of Photonics. John Wiley and Sons, New York, (1991).

[144] Yariv, A. Optical Electronics. Sunders HBJ, New York, (1991).

[145] Suzaki, Y. and Tachibana, A. Appl. Opt. 14, 2809 (1975).

[146] Wertheim, G. K., Butler, M. A., West, K. W., and Buchanan, D. E. Rev. Sci. Instrum. 45, 1369 (1974).

[147] Jolliffe, I. T. Principal Component Analysis. Springer, second edition, (2002). 